\title{
ESTUDO COMPARATIVO DA RESISTÊNCIA À COMPRESSÃO DO CIMENTO ÓSSEO NACIONAL E DO IMPORTADO, PREPARADOS MANUALMENTE E A VÁCUO
}

\author{
Carmen Aparecida Malaguti de Barros
}

Dissertação apresentada ao Programa de PósGraduação Interunidade em Bioengenharia Escola de Engenharia de São Carlos / Faculdade de Medicina de Ribeirão Preto / Instituto de Química de São Carlos da Universidade de São Paulo, para obtenção do título de Mestre em Bioengenharia.

ORIENTADOR: Prof. Dr. Celso H.F. Picado 
Barros, Carmen Aparecida Malaguti de

S277e Estudo comparativo da resistência à compressão do cimento ósseo nacional e do importado, preparados manualmente e à vácuo / Carmen Aparecida Malaguti de Barros. -- São Carlos, 2001.

Dissertação (Mestrado) -- Escola de Engenharia de São Carlos/Faculdade de Medicina de Ribeirão Preto/Instituto de Química de São Carlos-Universidade de São Paulo, 2001.

Área Interunidades : Bioengenharia.

Orientador: Prof. Dr. Celso H F Picado.

1. Polimetilmetacrilato (PMMA). 2. Cimento ósseo 3. Resistência à compressão do cimento. 4. Propriedades mecânicas do cimento ósseo. I. Título. 
Autora: Carmen Aparecida Malaguti de Barros

Título: Estudo comparativo da resistência à compressão do cimento ósseo nacional e do importado, preparados manualmente e a vácuo

Dissertação defendida e aprovada em pela comissão julgadora:

(Assinatura)

(Nome/Instituição)

(Assinatura)

(Nome/Instituição)

(Assinatura)

(Nome/Instituição)

Coordenador de Área 
A todos aqueles que se beneficiaram ou necessitam de cirurgia de substituição articular, a qual pode minimizar a sua dor, melhorar a função do membro comprometido e possibilitar-lhes uma melhor qualidade de vida.

"Quando lutamos por aquilo em que de fato acreditamos, conseguimos desenvolver o talento capaz de modificar o nosso próprio destino..." 
Agradeço a todos aqueles que direta e indiretamente contribuíram para a elaboração deste trabalho.

A Deus, força incondicional em todos os momentos da minha vida.

Ao meu orientador Prof. Dr. Celso H. F. Picado: "O professor se liga à eternidade, ele nunca sabe onde cessa a sua influência”. $\mathrm{O}$ meu eterno muito obrigado.

Aos meus familiares Cássio e Lucas, que me toleraram nesta jornada.

Ao Prof. Dr. Luiz Sérgio Marcelino Gomes, pelo incentivo à docência, à pesquisa e por todos os conhecimentos transmitidos.

Aos Professores Dr. Cláudio H. Barbieri e Dr. José Baptista Portugal Paulin, que tornaram possível minha participação no curso de Pós-graduação Interunidades Bioengenharia.

Ao Prof. Dr. Antônio Carlos Shimano, pela participação efetiva em todos os estágios do trabalho, orientações e conselhos em todas as etapas da pesquisa. estatística.

Ao Mestre Marcos Shimano, pela aplicação dos testes de análise

Ao engenheiro José Roberto Parpaioli, representante da Baumer Implantes Ortopédicos, pela cordialidade, amizade e dedicação.

Aos amigos e companheiros de curso Dyjalma Antônio Bassoli e Elisabete Dias Flauzino Gaspar Gomes.

Aos funcionários das Secretarias da Bioengenharia de São Carlos e Ribeirão Preto e Departamento de Biomecânica, Medicina e Reabilitação do Aparelho Locomotor da Faculdade de Medicina de Ribeirão Preto-USP, especialmente Janete, Terezinha, Fátima e Elisângela.

À Regina M. R. Pupin, pela amizade, paciência, traduções, digitação e formatação deste trabalho.

Aos diretores, docentes e funcionários da CEUCLAR, pela compreensão.

Agradeço também aqueles que foram importantes para a realização deste trabalho e que porventura tenha esquecido de citar. 


\section{SUMÁRIO}

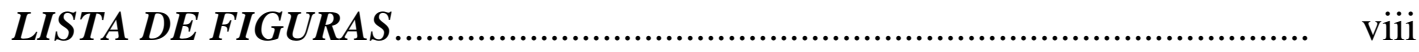

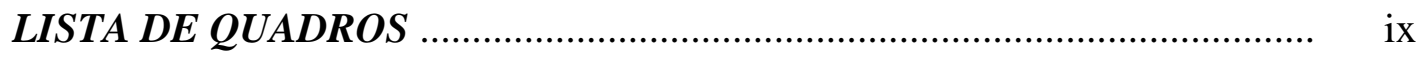

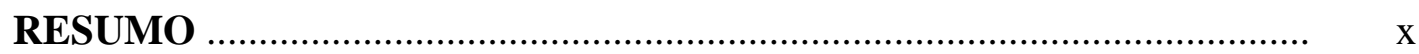

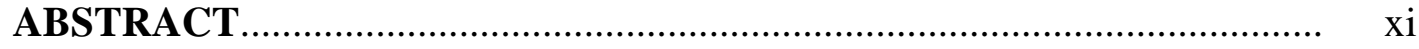

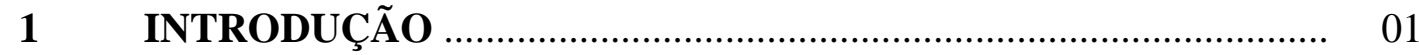

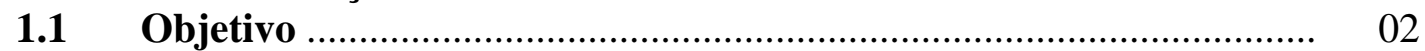

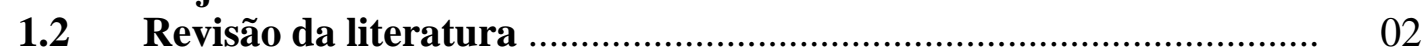

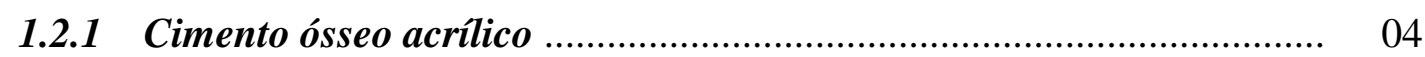

1.3 Métodos de mistura do cimento ósseo ............................................. 10

1.4 Propriedades mecânicas do cimento ósseo ......................................... 13

1.5 Revisão de conceitos gerais de ensaios mecânicos ............................. 14

1.5.1 Tensão aplicada ................................................................................... 15

1.5.2 Módulo de Young e Curvas de esforço versus deformação ................ 16

2 MATERIAIS E MÉTODOS ….................................................... 19

2.1 Preparação convencional ................................................................. 21

2.2 Preparação a vácuo ............................................................................ 23

2.3 Confecção do conjunto de moldagem ................................................ 25

2.4 Confecção dos corpos de prova........................................................... 27

2.5 Exame macroscópico ……………................................................ 29

2.6 Ensaios de compressão ............................................................. 30

2.6.1 Limite de proporcionalidade ......................................................... 33

2.7 Avaliação radiográfica da porosidade............................................. 34

2.8 Análise dos dados ........................................................................... 34

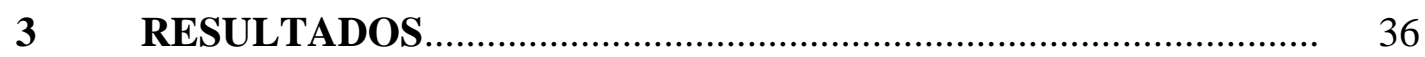

3.1 Ensaios de compressão ................................................................ 36

3.1.1 Módulo de elasticidade ......................................................................... 37

3.1.2 Tensão no limite de proporcionalidade ................................................ 39

3.1.3 Deformação no limite de proporcionalidade ........................................ 40

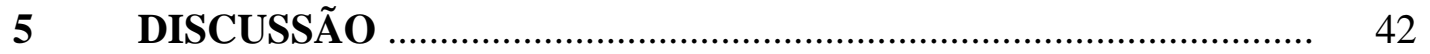




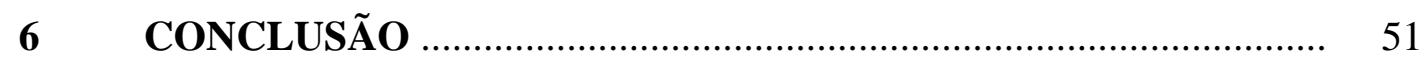

REFERÊNCIAS BIBLIOGRÁFICAS ……........................................ 52

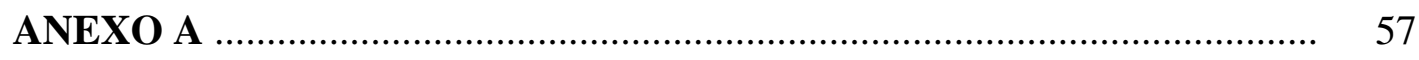

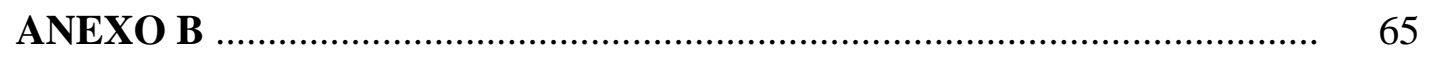

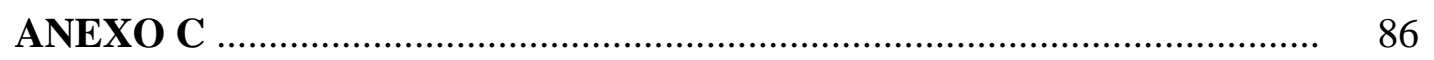

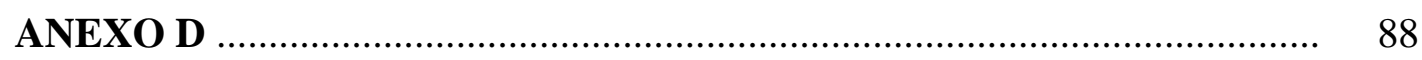

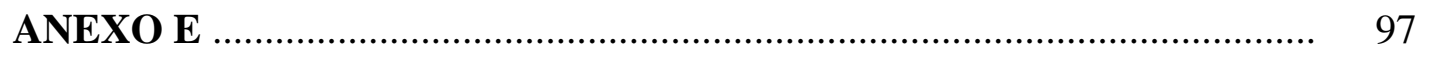

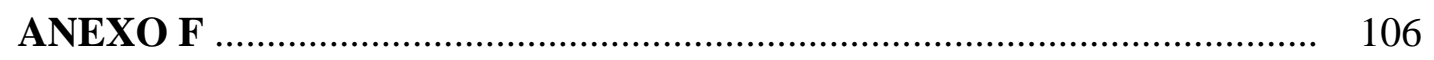

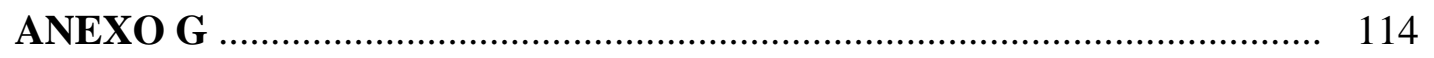

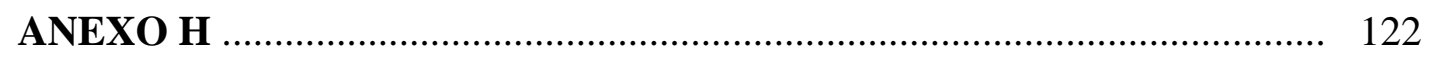

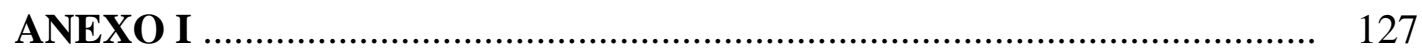

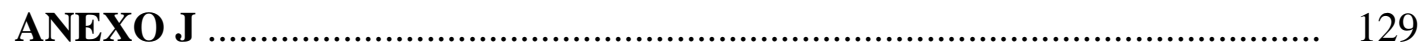

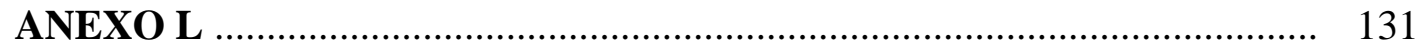




\section{LISTA DE FIGURAS}

FIGURA 1 Diagrama tensão versus deformação, segundo DANIELS, TOOMS \& HARKESS in CANALE, 1998

FIGURA 2 Material utilizado na técnica de mistura manual (1) cuba de aço inoxidável. (2) colher

FIGURA 3 Componentes do conjunto de cimentação a vácuo.

FIGURA 4 Técnica de preparação a vácuo.

FIGURA 5 Croqui do molde para confecção de corpos de prova para o ensaio de resistência à compressão, segundo a norma ASTM F451-86. Dimensões em milímetro.

FIGURA 6 Sistema de moldagem dos CPDs. $\left(\mathrm{A}_{1}\right.$ e $\left.\mathrm{A}_{2}\right)$ Placas lisas inferior e superior respectivamente. (B) Placa perfurada. (C) Pino removedor

FIGURA 7 Exemplos de corpos de prova e sua identificação

FIGURA 8 Dispositivos utilizados nos ensaios de compressão, a célula de carga (A) e o relógio comparador (B) com paralelismo entre as superfícies de ensaio

FIGURA 9 Detalhe de um CPD ao ser ensaiado..........................................

FIGURA 10 Gráfico tensão $x$ deformação, ilustrando a carga e a deformação no limite de proporcionalidade (ponto $\mathrm{C}$ ), a reta que representa a inclinação da curva $(\operatorname{tg} \alpha)$ e o triângulo formado pela união dos pontos (OBC).

FIGURA 11 Representação gráfica da curva tensão $x$ deformação para os quatro grupos experimentais ensaiados

FIGURA 12 Medianas do módulo de elasticidade dos quatro grupos experimentais

FIGURA 13 Valores das medianas da tensão no limite de proporcionalidade dos quatro grupos experimentais de cimento ósseo

FIGURA 14 Gráfico representativo das medianas das porcentagens de deformação dos quatro grupos de cimento 
LISTA DE QUADROS

QUADRO 1- Composição do cimento 1........................................................ 20

QUADRO 2- Composição do cimento 2 ….................................................. 21 
BARROS, Carmen Aparecida Malaguti de. Estudo comparativo da resistência à compressão do cimento ósseo nacional e do importado, preparados manualmente e a vácuo. Ribeirão Preto, 2001. 67 p. Dissertação (Mestrado em Bioengenharia) Escola de Engenharia de São Carlos, Faculdade de Medicina de Ribeirão Preto, Instituto de Química de São Carlos, Universidade de São Paulo.

\section{RESUMO}

O cimento ósseo, utilizado para a fixação de componentes protéticos nas cirurgias de substituição articular, mais resistente às forças de compressão do que às forças de tração, tem suas propriedades mecânicas alteradas por vários fatores entre

esses a formulação comercial e o método de preparação empregado. É o objetivo deste trabalho avaliar comparativamente as propriedades mecânicas à compressão de duas formulações comerciais de cimento ósseo preparadas manualmente e a vácuo, segundo as instruções do fabricante. Um conjunto de moldagem confeccionado em aço inoxidável permitiu preparar 48 corpos de prova para cada grupo experimental, totalizando 192 corpos de prova, que foram testados na Máquina Universal de Ensaios, tendo as especificações baseadas nas normas ISO 5833 e ASTM F451-86. A elaboração do diagrama tensão $x$ deformação de cada grupo experimental analisou as propriedades mecânicas do cimento ósseo quanto ao módulo de elasticidade, tensão e deformação no limite de proporcionalidade, entre grupos de mesma formulação comercial e entre os grupos com mesmo método de mistura. Analisados estatisticamente pelo método de Variança de Kruskal-Wallis $(p \leq 0,001)$ e pelo método de Dunn's $(p \leq 0,05)$. Quanto ao módulo de elasticidade, o grupo $2 \mathrm{M}$ foi o que apresentou maior módulo, $1563 \mathrm{MPa}$, valor estatisticamente significante ( $p \leq$ $0,05)$ em relação aos grupos $1 \mathrm{M}, 1 \mathrm{~V}$ e $2 \mathrm{~V}$. Para a tensão no limite de proporcionalidade os grupos $1 \mathrm{M}(39,40 \mathrm{MPa})$ e $2 \mathrm{~V}(39,65 \mathrm{MPa})$ foram os maiores valores de tensão no limite de proporcionalidade, não havendo diferença estatisticamente significante entre eles, mas essas diferenças foram significativas quando comparadas aos grupos $1 \mathrm{~V}$ e $2 \mathrm{M}$. A deformação no limite de proporcionalidade de maior valor percentual foi para o grupo $1 \mathrm{M}, 3,36 \%$, sendo esta diferença estatisticamente significante quando comparado ao grupo $2 \mathrm{M}$. Os testes de resistência à compressão do cimento nacionalizado e importado, preparados manualmente e a vácuo, mostraram não haver diferença importante entre os dois tipos de cimento ósseo, nem entre as duas formas de mistura-los.

Palavras-chave: polimetilmetacrilato (PMMA); cimento ósseo; resistência à compressão do cimento; propriedades mecânicas do cimento ósseo. 
BARROS, Carmen Aparecida Malaguti de. A comparable study of the compression resistance of the national and international bone cement prepared manually and by vacuum. Ribeirão Preto, 2001. 67 p. Dissertação (Mestrado em Bioengenharia) Escola de Engenharia de São Carlos, Faculdade de Medicina de Ribeirão Preto, Instituto de Química de São Carlos - Universidade de São Paulo.

\section{ABSTRACT}

The bone cement used for the fixation of the prosthetic components in the surgeries of joint replacement, more resistant to the compression than to the traction, has its mechanical properties altered by several factors among those the commercial formulation and preparation employee's method. It is the objective of this work to evaluate the mechanical properties to the compression of the two commercial formulas of bone cement manually prepared and using vacuum, according to the manufacturer's instructions. A molding set was made in stainless steel and it allowed for the preparation of 48 bodies of proof for each experimental group, adding up to 192 proof bodies total which were tested in the Universal Rehearsal Machine, with the specifications based on the ISO 5833 and the ASTM F451-86 regulations. The elaboration of the diagram "tension vs. deformation" of each of the experimental group analyzed the mechanical properties of the bone cement in relation to the elasticity module, tension and deformation on the proportional limit among the groups with the same commercial formula and among the groups with the same mixture method. Analyzed statistically, by Kruskal-Wallis's method of the variation $(\mathrm{p} \leq 0.001)$ and by the Dunn's method ( $\mathrm{p} \leq 0.05)$. Regarding the elasticity, the group $2 \mathrm{M}$ was the one with the highest module, $1563 \mathrm{MPa}$, a statistically significant value $(\mathrm{p} \leq 0.05$ ) in relation to the groups $1 \mathrm{M}, 1 \mathrm{~V}$ and $2 \mathrm{~V}$ to the tension at the limit of the proportionality, there is no significant differences among them, but these differences were relevant when compared to groups $1 \mathrm{~V}$ and $2 \mathrm{M}$. The deformation at the limit of the proportionality of the highest percentage was to group $1 \mathrm{M}, 3.36 \%$, being this the relevant statistical difference when compared to group $2 \mathrm{M}$. The resistance tests to the compression of the national and international cement prepared manually and by vacuum show that there is no important difference between the two kind of bone cement, and neither between the two ways of mixing them.

Key-words: polymethylmethacrylate (PMMA); bone cement; resistance to cement compression; mechanical proprieties of bone cement. 


\section{INTRODUÇÃO}

O cimento ósseo acrílico utilizado nas cirurgias de substituição articular não funciona como um adesivo, mas um espaçador entre a prótese e o osso, assegurando melhor distribuição de esforços, permitindo uma fixação estável do implante inicialmente e a longo prazo (NORMAN et al., 1995). O afrouxamento asséptico é uma das complicações mais freqüentes das próteses cimentadas, e vários questionamentos sobre a resistência do cimento ósseo ou fatores a ele relacionados têm sido estudados (JAMES et al.,1992; WANG et al.,1996; COMBS et al., 1979; DAVIES et al., 1988; ROSTOKER et al., 1979; GOODMAN et al., 1988; MULROY \& HARRIS, 1990; MOLINO \& TOPOLESKI, 1996; DAVIES et al., 1987).

Entre estes questionamentos, discutem-se os métodos de mistura do cimento ósseo que procuram diminuir a porosidade, aumentando assim sua resistência mecânica (LEE, LING \& WRIGHTON, 1973; RIMNAC et al.,1986; KRAUSE \& MATHIS, 1988; JAMES et al., 1992; MAJKOWSKI et al.,1993: GEIGER et al., 2001). 
Para os ortopedistas brasileiros, além de todos esses questionamentos em relação ao uso do cimento ósseo acrílico, discute-se também a maior ou menor adequação e a utilização do cimento embalado no Brasil em relação ao cimento ósseo proveniente de outros países. Sabe-se por intermédio de fontes não oficiais que muitos dos componentes do cimento ósseo são exportados, pelo Brasil a outros países, como, por exemplo, a Alemanha. Lá são manufaturados e posteriormente alguns dos seus componentes são importados por empresas brasileiras, que embalam e comercializam como cimento ósseo nacional. Os custos do cimento ósseo nacional ao consumidor são bem inferiores quando comparados aos custos dos cimentos importados, em valores percentuais estas cifras chegam a quase $25 \%$.

\subsection{Objetivo}

O objetivo deste trabalho foi o de comparar a resistência mecânica à compressão do cimento acrílico embalado no Brasil com a do cimento acrílico importado, ambos misturados tanto na forma manual tradicional como também sob vácuo.

\subsection{Revisão da literatura}


O polimetilmetacrilato, conhecido como cimento ósseo acrílico, foi patenteado na Alemanha em 1928, sendo produzido e comercializado por vários fabricantes com o nome Plexigás ${ }^{\circledR}$, Perspex ${ }^{\circledR}$ e Lucite $^{\circledR}$, entre outros. Inicialmente utilizado como biomaterial para formatar dentes artificiais e dentaduras, tornou-se conhecido na ortodontia como Paladon ${ }^{\circledR}$,ou como Polapont ${ }^{\circledR}$ (UNIVERSIDADE DE SÃO PAULO,1996)

Os cimentos ósseos aplicam-se em ortopedia em três funções principais: como massa para modelamento, sujeitos a tensões baixas; no preenchimento de cavidades ósseas, que, dependendo da localização, tais como articulações, ficam sujeitos a cargas elevadas; na fixação de próteses, em que o cimento forma uma interface entre a prótese e o osso e atua como homogeneizador e amortecedor de altas tensões, principalmente de compressão para fixação nas substituições articulares (UNIVERSIDADE DE SÃO PAULO, 1996; HAAS et al.,1975).

Sir John Charnley, em 1958, tornou-o popular entre os cirurgiões ortopédicos (CHARNLEY, 1960; DANIELS, TOOMS \& HARKESS in CANALE, 1998), utilizando-o inicialmente para fixar implantes nas substituições articulares do quadril, conhecida como a era moderna das artroplastias, sendo a cirurgia de substituição articular do quadril constituída de um componente femoral de aço inoxidável, onde era encaixada a cabeça femoral que se articulava com o componente acetabular de polietileno, de alta densidade, fixados ao osso pelo cimento de polimetilmetacrilato (PMMA). (KRAUSE, GRIMES \& MATHIS, 1988; LEWIS, 1999., BARB et al., 1982; SAHA \& PAL, 1984; JOHNSON et al., 1989; BISHOP, FERGUSON \& TEPIC, 1996; WIXSON et al., 1987; DEB, VASQUEZ \& BONFIELD, 1997). 
O uso do cimento ósseo acrílico para a fixação dos componentes protéticos nas artroplastias revolucionou o tratamento do estágio final das doenças articulares, graças aos excelentes resultados obtidos, comparados a outras técnicas utilizadas na época, tornando-se a técnica cirúrgica padrão das artroplastias (KRAUSE \& MATHIS, 1988).

\subsubsection{Cimento ósseo acrílico}

Existem dezenas de marcas comerciais de cimento ósseo. Elas podem ser divididas em duas categorias. O convencional ou pastoso um tipo de cimento que existe desde 1950 para uso ortopédico. O outro tipo é o de baixa viscosidade, desenvolvido na década de 80, para ser utilizado com pistola injetora, facilitando o completo preenchimento do canal medular.

Geralmente, os cimentos ósseos são comercializados em pacotes de $40 \mathrm{~g}$ de polimetilmetacrilato em pó e $20 \mathrm{ml}$ de monometilmetacrilato líquido. A sua obtenção ocorre a partir da polimerização por adição de radicais livres em suspensão ou massa, do monômero metacrilato de metila e do polímero em pó a partir das cadeias moleculares apresentadas a seguir: monômero $=\mathrm{n}(\mathrm{HC}=\mathrm{C}(\mathrm{CH})(\mathrm{COOCH}))$; polímero $=(\sim \mathrm{HC}-\mathrm{C}(\mathrm{CH}) \mathrm{COOCH}) \sim) \mathrm{n} . \mathrm{O}$ cimento em pó consiste basicamente de ligações poliméricas, com partículas de tamanho que varia de 30 a $150 \mu$ de diâmetro, composto pelas substâncias e representação química enumeradas abaixo: 
Poli(metilmetacrilato)

$$
\left(\sim \mathrm{H}_{2} \mathrm{C}-\mathrm{C}\left(\mathrm{CH}_{3}\right)\left(\mathrm{COOOCH}_{3}\right) \sim\right) \mathrm{n}
$$

Peróxido de benzoíla(BPO)

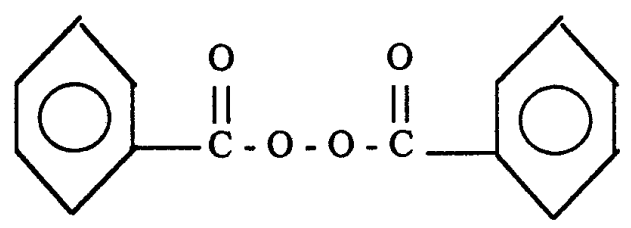

Poli(metilmetacrilato-estireno) sendo o estireno um componente minoritário deste copolímero.

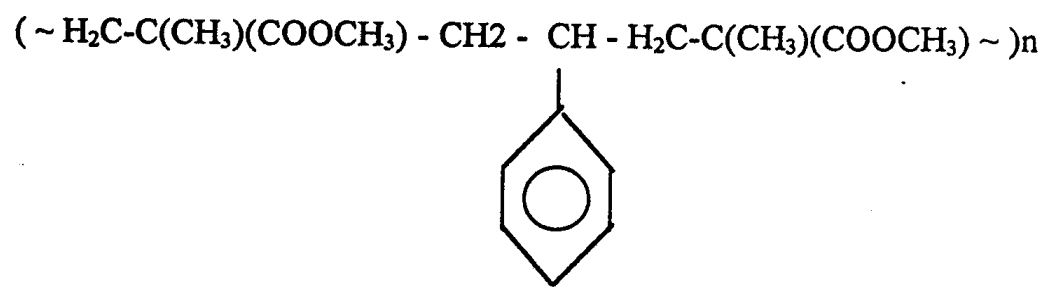

Dois aditivos radiopacos são acrescentados o Sulfato de bário ou o Óxido de zircônio (zircônia)-ZrO

Na composição do monômero líquido encontram-se as substâncias listadas abaixo e a sua fórmula química:

Metacrilato de metila

$$
\mathrm{H}_{2} \mathrm{C}=\mathrm{C}\left(\mathrm{CH}_{3}\right)\left(\mathrm{COOCH}_{3}\right)
$$

Álcool Etílico

$$
\mathrm{C}_{2} \mathrm{H}_{5} \mathrm{OH}
$$


Ácido Ascórbico

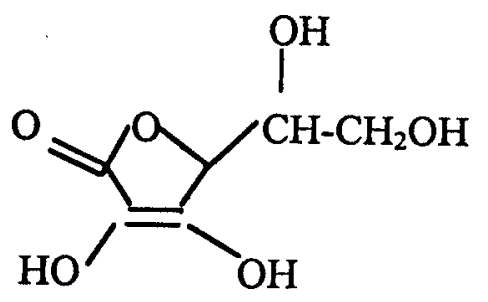

Hidroquinona

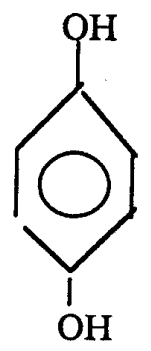

$\mathrm{O}$ agente ativador da polimerização N,N dimetil-p-toluidino é encontrado na proporção de $2 \%$ da massa. Há também composições nas quais se acrescentam antibióticos como a gentamicina ou a vancomicina, para a prevenção ou tratamento de infecções (ASKEW et al., 1990; CHAN, AHMED \& JOHNSON in MORREY, 1996).

$\mathrm{N}, \mathrm{N}$ dimetil-p-toluidino

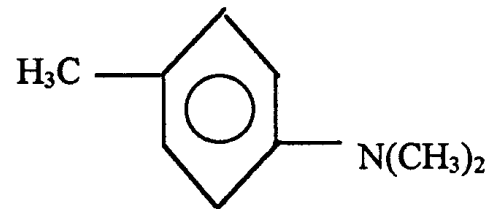


Durante o preparo, o cimento ósseo acrílico passa por três estágios distintos, variando suas propriedades físicas e químicas, permitindo assim manuseá-lo ou não, dependendo da fase de polimerização em que se encontra.

A mistura do monômero e do polímero pode ser feita manualmente com espátula, através do misturador a vácuo, sob centrifugação, agitação ultra-sônica ou combinando-se os sistemas. Ao se misturarem o pó e o líquido, o ativador e o iniciador reagem entre si, formando radicais livres que iniciam a polimerização em massa (CHAN, AHMED \& JOHNSON in MORREY , 1996). A partir da mistura dos componentes chamada de tempo zero $\left(\mathrm{t}_{0}\right)$, o cimento apresenta-se sob uma forma viscosa denominada pré-massa, no intervalo de tempo entre $t_{0}$ e $t_{1}$, há um aumento de sua viscosidade com o decorrer da polimerização.

Quando a mistura adquire consistência suficiente para ser manuseada, não grudando mais às luvas do cirurgião, considera-se que atingiu o tempo de formação de massa $\left(t_{1}\right)$, permitindo assim o seu manuseamento. Entre $t_{1}$ e $t_{2}$, a mistura é denominada de massa, há um aumento da temperatura da mistura, sendo a reação de polimerização exotérmica, ocorre então uma rápida elevação do grau de conversão e do peso molecular do polímero, resultado da reação e do processo de auto-aceleração das polimerizações em massa. A temperatura alcança valores entre $67^{\circ} \mathrm{C}$ e $124^{\circ} \mathrm{C}$, com um valor médio próximo a $90^{\circ} \mathrm{C}$.

O cimento convencional permanece no estado de pré-massa por 1 a 2 minutos antes de entrar na fase de massa, que varia de 3 a 7 minutos, até seu endurecimento total, entre 8 e 14 minutos, dependendo da marca comercial do cimento e das condições ambientais. Os cimentos de baixa viscosidade mantêm-se no estado de massa por 5 minutos e endurecem entre 6 e 8 minutos. Contudo, os 
cimentos do tipo convencional e os de baixa viscosidade são afetados pela temperatura, pela umidade relativa do ar e pelas condições de armazenamento de forma similar. Ambientes quentes e úmidos diminuem a fase de massa, antecipando o endurecimento do cimento ósseo em até 5 minutos. $\mathrm{O}$ aumento da temperatura em $1{ }^{\circ} \mathrm{C}$ pode acelerar o endurecimento do cimento em 1 minuto. $\mathrm{O}$ armazenamento do cimento em um ambiente úmido por período prolongado sofre efeitos similares (CHAN, AHMED \& JOHNSON in MORREY, 1996).

Com a utilização das artroplastias em pacientes jovens e ativos, houve a necessidade de serem desenvolvidos novos materiais para a confecção dos implantes, com novos desenhos e técnicas cirúrgicas apropriadas.

O conceito de artroplastia de baixo atrito, associado à cimentação por meio de polimetilmetacrilato (PMMA), marcou o início da era moderna das artroplastias, descritas como técnicas de cimentação de primeira geração. A técnica preconizada por Charnley, em 1958, chamada de técnica de cimentação de primeira geração envolvia a mistura em ambiente cirúrgico, com introdução manual do cimento em estado pastoso no canal femoral e no acetábulo. Os resultados iniciais foram estimulantes, mas com o passar do tempo, começaram a apresentar altos índices de complicações, como afrouxamento acetabular e femoral. Estas complicações aumentavam em grandes proporções, em pacientes jovens e nas cirurgias de revisão (MULROY \& HARRIS, 1990; MULROY, ESTOK \& HARRIS, 1995).

Clinicamente, o afrouxamento causa reabsorção óssea, fratura óssea, separação da interface cimento-osso e/ou cimento-prótese e fratura do manto de cimento ósseo. (BURKE, GATES \& HARRIS, 1984; LEWIS, 1997; CHAN, AHMED \& JOHNSON in MORREY , 1996; LEWIS, NYMAN \& TRIEU, 1998). 
O aperfeiçoamento das técnicas de cimentação, iniciado em 1972, chamadas de técnicas de cimentação de segunda geração, passa a utilizar cimentos de baixa viscosidade, introduzidos com a pistola de injeção retrógrada e a utilização dos tampões de canal medular no lado femoral, enquanto outros instrumentos pressionam o cimento no lado acetabular. As técnicas de cimentação de segunda geração procuram produzir ao redor do implante uma manta uniforme e completa de cimento, sem falhas, que é pressionado de modo a interdigitar-se com o osso (SCHURMAN et al., 1989; MULROY \& HARRIS, 1990; MULROY, ESTOK \& HARRIS, 1995).

Segundo LEWIS \& NYMAN (2000), o afrouxamento é iniciado pela fragmentação do cimento que leva à osteólise. Predispõe a fragmentação à presença de cantos vivos nas próteses, camada de cimento fina ou incompleta e a presença de porosidade no cimento ósseo.

A eliminação do cimento ósseo e a utilização das próteses não cimentadas, na década de 1980, foram marcadas pela discussão entre próteses cimentadas e não cimentadas; no que diz respeito à osteólise, os componentes não cimentados demonstraram reabsorção óssea mais precoce, mais freqüente, mais intensa e progressiva em relação à prótese cimentada. Cai, portanto, a teoria que tenta provar que, eliminando-se o cimento ósseo, elimina-se a osteólise, chamada também, inicialmente, de doença da prótese não cimentada.

Ao mesmo tempo em que se desenvolviam as próteses não cimentadas, procuravam-se também corrigir as possíveis causas do afrouxamento das próteses cimentadas, através da preparação mais adequada do estojo ósseo a ser cimentado, da aplicação de técnicas que diminuíssem o sangramento do canal femoral no momento da cimentação, da lavagem adequada do canal femoral para evitar que a gordura e 
restos de tecidos fossem misturados ao cimento ósseo e também impedir a formação de bolhas durante o processo de mistura e aplicação do cimento ósseo. Uma outra causa freqüentemente relatada de afrouxamento é a necrose óssea (COMBS \& GRENWALD, 1979; BARB et al., 1982. CHIN, STAUFFER \& CHAD, 1990).

Diferentes técnicas foram pesquisadas na tentativa de melhorar a qualidade da cimentação, desenvolveram-se equipamentos sofisticados, e dentre estas técnicas denominadas de terceira geração, destacamos a mistura a vácuo do cimento ósseo (WIXSON, LAUTENSCHLAGER \& NOVAK, 1985) e a centrifugação (BURKE, GATES \& HARRIS, 1984). O afrouxamento asséptico dos componentes é a causa mais freqüente de complicações a longo prazo das artroplastias cimentadas (SCHURMAN et al., 1989; MULROY \& HARRIS, 1990).

A durabilidade das cirurgias de substituição articular, principalmente nas articulações de suporte de carga, depende da combinação de vários fatores, os quais incluem: o peso e a atividade do paciente, o modo como os componentes são apoiados pelo cimento ósseo ou osso, o desenho, o tipo e a posição anatômica dos componentes e o tipo de metal utilizado (HARKESS in CANALE, 1998).

\subsection{Métodos de mistura do cimento ósseo}

Os métodos de mistura do cimento ósseo são classificados por LEWIS (1997) em: mistura manual, mistura a vácuo, centrifugação, agitação ultra-sônica e a combinação mecânica de misturas. 
No método de mistura manual, o componente em pó é adicionado ao componente líquido (este componente pode ou não estar resfriado a uma temperatura usualmente entre $15^{\circ} \mathrm{C}$ e $6^{\circ} \mathrm{C}$ ) em uma tigela polimérica (usualmente polipropileno) ou em uma cuba metálica. Os componentes são misturados com uma espátula de polipropileno a uma velocidade de 1 ou $2 \mathrm{~Hz}$ pelo tempo de 45 a 120 segundos. A mistura do cimento ósseo manualmente, nas condições atmosféricas, permite a entrada de ar nas cadeias de polímeros, e a sua viscosidade natural permite que apenas as grandes bolhas migrem para a superfície, deixando um número considerável de bolhas menores que $1 \mathrm{~cm}$ (CHAN, AHMED \& JOHNSON in MORREY , 1996).

Na centrifugação, os componentes do cimento ósseo previamente adicionados um ao outro manualmente são imediatamente colocados em uma seringa, levados a uma centrífuga a 2300-4000 rpm, pelo tempo de 30 a 180 segundos.

Na mistura a vácuo, o componente em pó normalmente é adicionado ao componente líquido, o vácuo é aplicado em pressões que variam de 5 para $100 \mathrm{kPa}$, movimentado a uma velocidade de 1 ou $2 \mathrm{~Hz}$, por um espaço de tempo que varia entre 15 e 150 segundos (CHAN, AHMED \& JOHNSON in MORREY, 1996).

CHAN, AHMED \& JOHNSON in MORREY (1996), WANG et al. (1996), WIXSON, LAUTENSCHLAGER \& NOVAK (1987), KURDY, HODGKINSON \& HAYNES (1996) e GEIGER et al. (2001) estabeleceram que a mistura a vácuo, utilizando-se uma pressão atmosférica entre 400 a $730 \mathrm{mmHg}$, reduz significativamente a porosidade de $5 \%$ a $10 \%$ para menos de $1 \%$. Essa redução da porosidade é resultado da eliminação das macro e microporosidades, sendo efetiva para todos os tipos de cimento ósseo. O nível de vácuo recomendado é de 500-550 
mmHg sobre a pressão atmosférica.

SCHEURS et al. (1988), ao avaliarem quatro técnicas de preparação: mistura manual, pressurização com pistola pneumática, centrifugação e mistura a vácuo, em três cimentos de viscosidade diferentes, encontraram uma redução da porosidade de 60-80\% quando comparados a mistura manual.

A agitação ultra-sônica um outro método de mistura no qual o componente em pó é adicionado ao líquido em uma cuba de aço inoxidável e posteriormente é colocado em um prato vibratório (50 vibrações/segundo), enquanto é misturado com uma espátula de propileno (CHAN, AHMED \& JOHNSON in MORREY, 1996). Outros aparelhos também podem ser usados para misturar o cimento, como exemplo citamos um motor acoplado a uma unidade excêntrica que faz movimento em duas direções, ou então uma máquina que mistura e centrifuga simultaneamente (LEWIS, 1997).

Todos esses sistemas ou métodos foram desenvolvidos, portanto, para minimizar a porosidade do cimento ósseo que é ocasionada pela inclusão de ar nos espaços entre as cadeias de polímero, envolvendo uma série de mecanismos, que podem ou não estar associados:

1) entrada de ar durante o método de mistura e sua transferência;

2) presença de ar nos espaços entre as cadeias de polímero;

3) geração de bolhas, que é resultado da evaporação ou ebulição do monômero;

4) expansão térmica de bolhas;

5) presença de cavitação das bolhas (WIXSON, LAUTENSCHLAGER \& NOVAK, 1987; CHAN, AHMED \& JOHNSON in MORREY, 1996).

A porosidade pode ser mensurada como um percentual de volume ou como 
um percentual de área de seção transversal ocupada pelas bolhas.

Esses espaços ocupados por ar são classificados em duas categorias, de acordo com o seu tamanho em diâmetro, as microporosidades, com diâmetros que variam entre $(0,1$ e $1 \mathrm{~mm})$ e as macroporosidades com diâmetros superiores a $1 \mathrm{~mm}$.

Os valores de porosidade do cimento ósseo misturados manualmente, relatados pela literatura, variam entre $5 \%$ e $16 \%$ (CHAN, AHMED \& JOHNSON in MORREY, 1996) e 9 a 27\% segundo DANIELS, TOOMS \& HARKESS in CANALE (1998).

\subsection{Propriedades mecânicas do cimento ósseo}

As artroplastias do quadril são submetidas às forças de tração em vários locais, sendo a face lateral do implante um exemplo clássico, em razão da sua própria angulação. Os valores da força de tensão máxima, módulo de elasticidade (E) e a tensão no limite de proporcionalidade $\left(\mathrm{e}_{\max }\right)$ são obtidos da curva de tensão versus deformação, que cresce linearmente, sendo o cimento ósseo classificado como um material frágil (LEWIS, 1997).

A postura de pé bipodal ou monopodal submete o aparelho locomotor a forças compressivas diretas, que podem ser estáticas ou quase estáticas. Nas substituições articulares o manto de cimento que se interpõe entre o osso e a haste femoral passa a ser uma cunha compressiva, agindo como um amortecedor e absorvedor de choque entre o implante e o osso. Portanto, as propriedades 
compressivas quase estáticas são relevantes para o cimento ósseo (LEWIS, 1997).

A determinação in vitro destas propriedades é feita de acordo com as especificações das normas ASTM F451 (Anexo A) e a ISO 5833 (Anexo B). Os valores da força de tensão máxima e o módulo de elasticidade são estimados das curvas de tensão versus deformação, usando-se a regra de $2 \%$ de compensação e a inclinação da seção reta inicial da curva, respectivamente. $O$ limite de proporcionalidade que corresponde ao ponto $\mathrm{C}$ da curva tensão $x$ deformação é dado pelos valores da tensão/deformação neste ponto (LEWIS, 1997).

Os valores da força de tensão máxima, módulo de elasticidade e tensão no limite de proporcionalidade variam, de acordo com as várias formulações de cimento ósseo, segundo duas tendências. A primeira, que para um dado método de mistura a formulação exerce uma influência profunda sobre alguns parâmetros, como: as quantidades de estabilizadores, iniciadores e radiopacificadores; a presença ou ausência de co-polímero; o tamanho e a distribuição do pó; a proporção entre monômero e o polímero; e a taxa de mudança de viscosidade com o tempo. A segunda tendência é que o método de mistura é importante possivelmente por seu efeito sobre micro e macroporosidades (LEWIS, 1997).

CHAN, AHMED \& JOHNSON in MORREY, em 1996, apresentam valores médios de algumas propriedades mecânicas do polimetilmetacrilato: tensão de tração igual a $32 \mathrm{MPa}$, tensão de compressão igual a 100 Mpa e módulo de elasticidade igual a $2700 \mathrm{Mpa}$.

\subsection{Revisão de conceitos gerais de ensaios mecânicos}


As propriedades mecânicas definem o comportamento de um material quando sujeito a esforços de natureza mecânica e correspondem às propriedades que, num material especificado, determinam a sua capacidade de transmitir e resistir aos esforços que lhes são aplicados sem romper ou sem que produzam deformação.

Os ensaios mecânicos de materiais envolvem a aplicação de forças conhecidas e a medida da deformação produzida. As forças podem ser divididas basicamente em relação ao sentido de aplicação: tração, torção e flexão, compressão, cisalhamento.

\subsubsection{Tensão aplicada}

Quando um corpo é submetido a uma força, ele está exposto a uma deformação pela ação dessa mesma força, força essa chamada de tensão. A magnitude da força, sua duração e a direção é que determinam a extensão da deformação.

Tensão pode ser definida, portanto, como a razão entre a força atuante em um determinado sistema e a área de seção transversal do corpo submetido à atuação daquela força. Essa relação é expressa da seguinte forma:

$$
\tau=\stackrel{F}{ }
$$


onde: $\tau=$ tensão $\left(\mathrm{kgf} / \mathrm{cm}^{2}\right.$ ou N/$\left./ \mathrm{cm}^{2}\right)$

$$
\begin{aligned}
& \mathrm{F}=\text { força }(\mathrm{kgf} \text { ou } \mathrm{N}) \\
& \mathrm{A}=\operatorname{área}\left(\mathrm{m}^{2}, \mathrm{~cm}^{2} \text { ou } \mathrm{mm}^{2}\right) .
\end{aligned}
$$

Algumas características próprias dos materiais são importantes para determinar o aparecimento de deformações, como, por exemplo, a capacidade de absorção de energia e o módulo de elasticidade (módulo de Young).

\subsubsection{Módulo de Young e Curvas de esforço versus deformação}

A lei de Hooke, que mostra a relação de linearidade entre força e deformação de um objeto sólido, é válida somente na fase elástica dos materiais quando submetidos ao carregamento, que é dado por:

$$
\tau=\mathrm{E} . \varepsilon,
$$

em que: $\tau=$ tensão aplicada;

E = módulo de elasticidade, também conhecido como módulo de Young; $\varepsilon=$ deformação exercida no material.

A primeira porção da curva é linear e a deformação aumenta 
proporcionalmente ao esforço, até que o ponto de cessão ou limite de proporcionalidade seja alcançado e denote o final da fase elástica. Se em qualquer ponto da curva antes deste ponto a carga é removida, a substância retorna à sua forma de repouso. A inclinação desta curva gera um ângulo que é a medida da rigidez do material. Quanto maior a tangente do ângulo, maior a rigidez do material; a tangente do ângulo é denominada de módulo de elasticidade ou módulo de Young.

O trecho da curva do ponto que une o limite de proporcionalidade ao ponto de ruptura mostra que a deformação aumenta mais rapidamente com o aumento da força aplicada. Esta é a região plástica da curva onde uma deformação permanente é produzida no material. Os materiais conhecidos como dúcteis podem ou não absorver muita energia na fase elástica, porém, rompem-se logo que entram na fase plástica, como exemplo citamos o vidro. Já os materiais frágeis absorvem energia na fase elástica, porém, rompem-se logo que entram na fase plástica denominada de deformação plástica , tendo como exemplo o cimento ósseo acrílico. Esses dados são ilustrados pela Figura 1.

Com a continuidade de aplicação de carga, um esforço máximo será alcançado, chamado de resistência final à tração (RFT) ou quebra do material, representando o esforço ou estresse máximo que o material é capaz de absorver (Figura 1).

Com a análise da força e das deformações ocorridas no material durante o ensaio mecânico e o conhecimento da geometria do material, pode-se construir a curva tensão $x$ deformação que fornece as principais características do material a ser analisado. 


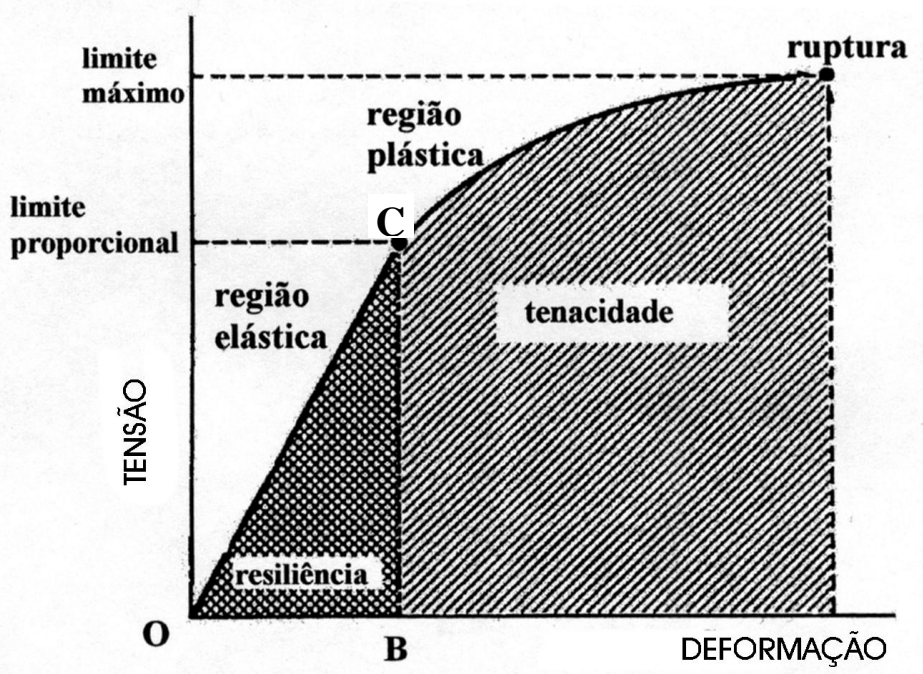

FIGURA 1 - Diagrama tensão versus deformação, segundo DANIELS, TOOMS \& HARKESS in CANALE, 1998. 


\section{MATERIAL E MÉTODOS}

Para a preparação dos corpos de prova (CPDs) experimentais foram utilizadas duas marcas comerciais de cimento ósseo, uma nacional ${ }^{1}$ e outra importada $^{2}$, sendo ambas do tipo pastoso e preparadas de acordo com as normas ASTM F451/86 (Anexo A) e ISO 5833 (Anexo B), que serão detalhadas durante as fases do experimento.

Os cimentos de ambas as marcas, assim como o conjunto de cimentação a vácuo (Howmédica, Ireland), foram doados ao Departamento de Biomêcanica, Medicina e Reabilitação do Aparelho Locomotor da Faculdade de Medicina de Ribeirão Preto-USP pelas empresas que embalam, comercializam e revendem estes produtos no país, respectivamente Baumer Implantes Ortopédicos, Mogi Mirim e MCI Implantes, Campinas.

Um grupo experimental piloto foi preparado para adaptar todas as condições exigidas pelas normas: condições ambientais, instrumentação e técnica adequadas aos dois tipos de mistura, tempo de mistura e de transferência do cimento ósseo para o conjunto de moldagem, remoção dos CPDs do conjunto de moldagem,

\footnotetext{
${ }^{1}$ Baumer Osteo-Class (Baumer S/A).

${ }^{2}$ Simplex P (Howmédica, Ireland).
} 
armazenamento e padronização dos testes de compressão dos CPDs no Departamento de Biomêcanica, Medicina e Reabilitação do Aparelho Locomotor da Faculdade de Medicina de Ribeirão Preto-USP.

Quatro grupos experimentais de corpos de prova foram preparados:

- Grupo 1M: cimento nacional, preparado manualmente;

- Grupo 1V: cimento nacional, preparado a vácuo;

- Grupo 2M: cimento importado, preparado manualmente;

- Grupo 2V: cimento importado, preparado a vácuo.

As composições dos cimentos ósseos dos grupos 1 e 2 são apresentadas nos Quadros 1 e 2.

QUADRO 1. Composição do Cimento 1.

Uma ampola contendo 18,37 g $(20 \mathrm{ml})$ de monômero líquido é constituída de:

- Monometilmetacrilato

$-17,87 \mathrm{~g}(97,278 \%)$

- N,N-dimetil-paratoluidino

- $0,50 \mathrm{~g}(2,722 \%)$

- Hidroquinona

- $0,0004 \mathrm{~g}(0,002 \%)$

O componente líquido é esterilizado por ultrafiltragem.

O outro componente, um envelope contendo $40 \mathrm{~g}$ de polímero, consiste de:

- Polimetilmetacrilato - 38,50 g $(91,25 \%)$

- Peróxido residual $\quad-\quad 0,5 \mathrm{~g}(1,25 \%)$

- Zircônia $\quad-3,0 \mathrm{~g}(7,50 \%)$

O componente em pó é esterilizado por óxido de etileno. 
QUADRO 2. Composição do Cimento 2.

Uma ampola contendo $20 \mathrm{ml}$ do monômero líquido é constituída de:

- Metacrilato de metila (monômero) - 19,5 ml

- N,N-dimentil-paratoluidino $\quad-0,5 \mathrm{ml}$

- Hidroquinona - $1,5 \mathrm{mg}$

O componente líquido é esterilizado por filtração através de membrana.

O outro componente, um envelope de $40 \mathrm{~g}$ de polímero, consiste de:

- Copolímero de metacrilato de metila-estireno - 30,0 g

- Polimetacrilato de metila - $6,0 \mathrm{~g}$

- Sulfato bárico - 4,0 g

O componente em pó é esterilizado por raios gama.

As condições ambientais para a realização do experimento, normatizadas pelas ASTM F 451-86 e ISO 5833, estabelecem um padrão de ambientação que consiste na manutenção da temperatura a $21 \pm 2{ }^{\circ} \mathrm{C}$ e umidade relativa do ar de $80 \pm$ $10 \%$. Estas condições ambientais foram mantidas por 24 horas antes do preparo; durante a sua preparação, $24 \pm 2$ horas após o preparo e durante a execução dos testes de compressão. Para monitorar estas duas condições foi utilizado um termohigrômetro digital (Thermo-Hygro ${ }^{\circledR}$ ).

\subsection{Preparação convencional}

As preparações dos dois grupos de cimento ósseo convencional foram realizadas em uma sala do Departamento de Biomecânica, Medicina e Reabilitação do Aparelho Locomotor da Faculdade de Medicina de Ribeirão Preto-USP, com a temperatura e a umidade relativa controladas. As condições ambientais utilizadas simulam o ambiente de um centro cirúrgico. 
A mistura manual foi realizada de acordo com as prescrições do manual de instruções das marcas comerciais de cimento ósseo utilizadas pelo experimento.

Para a preparação convencional dos cimentos ósseos foram utilizadas uma cuba de aço inoxidável e uma colher de cozinha estéreis (Figura 2).

Os pacotes de cimento dos grupos 1 e 2 foram abertos, e o componente em pó (polímero) depositado na cuba. Em seguida, o componente líquido (monômero) foi despejado sobre o pó, misturado com o cabo da colher de cozinha, com movimentação leve, até formar uma massa homogênea, que não se prendesse mais às luvas do manipulador. Cerca de 1,5 minutos após o início da mistura, iniciou-se a verificação do tempo de formação de massa: a cada 15 segundos o dedo indicador, recoberto por luva cirúrgica sem pó ou talco, era imerso na mistura, até que não houvesse aderência entre a luva e o cimento ósseo, cerca de 3 minutos após o início da mistura.

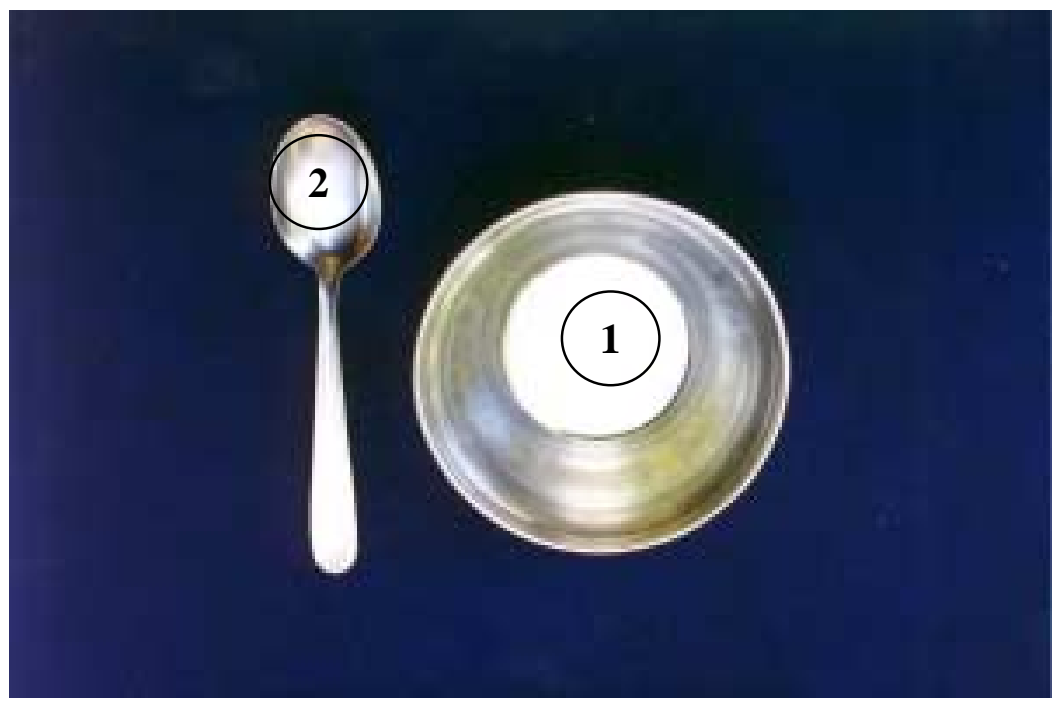

FIGURA 2. Material utilizado na técnica de mistura manual. (1) cuba de aço inoxidável. (2) colher. 


\subsection{Preparação a vácuo}

Para a preparação a vácuo foi necessário um treinamento preliminar, fornecido pela empresa que comercializa o conjunto desta preparação, obedecendose rigorosamente às instruções do fabricante. A mistura a vácuo dos grupos 1 e 2 se fez nas mesmas condições ambientais já descritas anteriormente para a mistura manual, sendo necessária uma sala onde houvesse uma saída de vácuo. A pressão atmosférica máxima alcançada foi de 300-350 $\mathrm{mmHg}$, condição freqüente na maioria das salas cirúrgicas dos hospitais brasileiros.

O conjunto de cimentação era descartável (Figura 3), composto por um copo plástico (A) em que foi colocado o componente líquido e sobre ele o componente em pó. Uma espátula confeccionada em metal com um cabo plástico (B) foi utilizada para promover a homogeneização adequada dos componentes do cimento ósseo; sendo o tempo gasto neste processo o suficiente para permitir a homogeneização do polímero e do monômero. A seguir, o copo, contendo a mistura homogeneizada, foi encaixado em uma base de plástico (C), que apresentava inferiormente uma saída para a mangueira de vácuo previamente ajustada à saída de vácuo da parede da sala cirúrgica; a tampa superior da base foi acoplada ao sistema (D) e, depois de checada a conexão das saídas, o cimento ósseo foi movimentado por duas hastes finas e fixas confeccionadas em metal (E), acopladas à tampa superior e movimentadas por meio de uma manivela confeccionada em plástico $(\mathrm{F})$, que permitia a movimentação a uma velocidade de um ciclo por segundo, ou seja, 60 ciclos por minuto, durante um 1 min e $30 \mathrm{~s}$, conforme as instruções do fabricante. 
Contados 1 min e $30 \mathrm{~s}$, removiam-se a mangueira da base do misturador, a tampa superior, o copo, e o cimento ósseo, ainda pastoso, era transferido para o conjunto de moldagem.

A Figura 4 ilustra o conjunto de cimentação utilizado por este trabalho, já acoplado para permitir a mistura a vácuo dos cimentos ósseos dos grupos 1 e 2 .

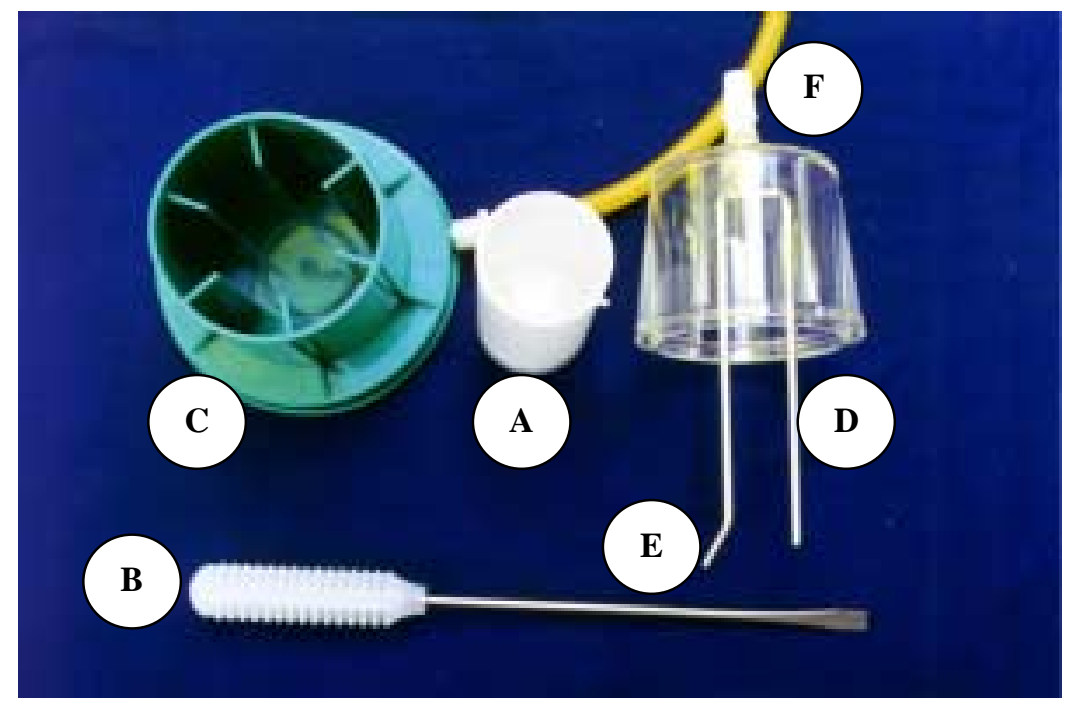

FIGURA 3. Componente do conjunto de cimentação a vácuo.

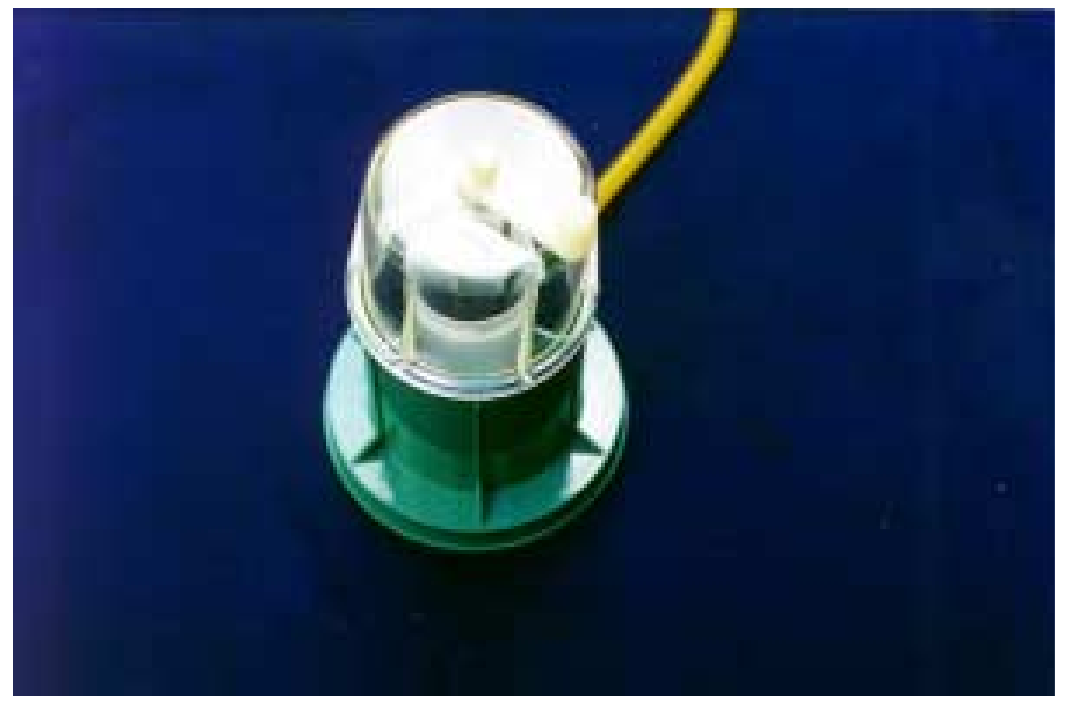

FIGURA 4. Técnica de preparação a vácuo. 


\subsection{Confecção do conjunto de moldagem}

O conjunto de moldagem foi confeccionado segundo a norma ASTM.F45186 (Anexo A). Para que isso se tornasse possível, foram realizadas várias reuniões entre os pesquisadores e os técnicos da Indústria de Implantes Ortopédicos Baumer S.A., até que se chegasse ao projeto do conjunto de moldagem, que foi usinado em aço inoxidável pela indústria anteriormente citada e doado ao Departamento de Biomecânica, Medicina e Reabilitação do Aparelho Locomotor da Faculdade de Ribeirão Preto-USP, pela Indústria de Implantes Ortopédicos Baumer S.A.

O sistema é composto por duas placas maciças iguais e lisas e uma intermédiaria perfurada, medindo 76,2 \pm 0,2 $\mathrm{mm}$ de diâmetro e 12,0 $\pm 0,1 \mathrm{~mm}$ de altura. Uma das placas lisas era posicionada inferiormente, funcionando como base $\left(A_{1}\right)$, sobre ela era posicionada a placa perfurada com 48 orifícios de $6,0 \pm 0,1 \mathrm{~mm}$ de diâmetro e superiormente a outra placa lisa posicionada sobre as outras duas, funcionando como uma tampa (A2).

Para facilitar a remoção dos 48 corpos de prova, foi confeccionado um pino removedor com diâmetro de 5,0 $\mathrm{mm}$ e comprimento de 5,0 cm, confeccionado em aço inoxidável (Figura 5).

A Figura 6 ilustra o conjunto de moldagem elaborada para a confecção dos corpos de prova, duas bases maciças $\left(\mathrm{A}_{1}\right.$ e $\left.\mathrm{A}_{2}\right)$, uma placa perfurada (B), compressão do conjunto de moldagem na morsa (C) e pino removedor (D). 


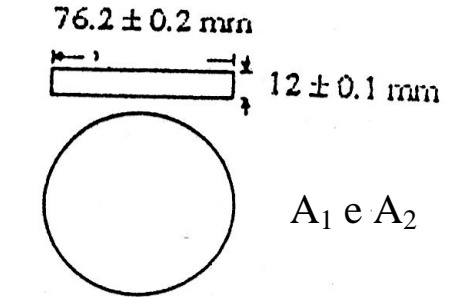

Placa supericur c inferios. (ṇ:o iressidauc:) )

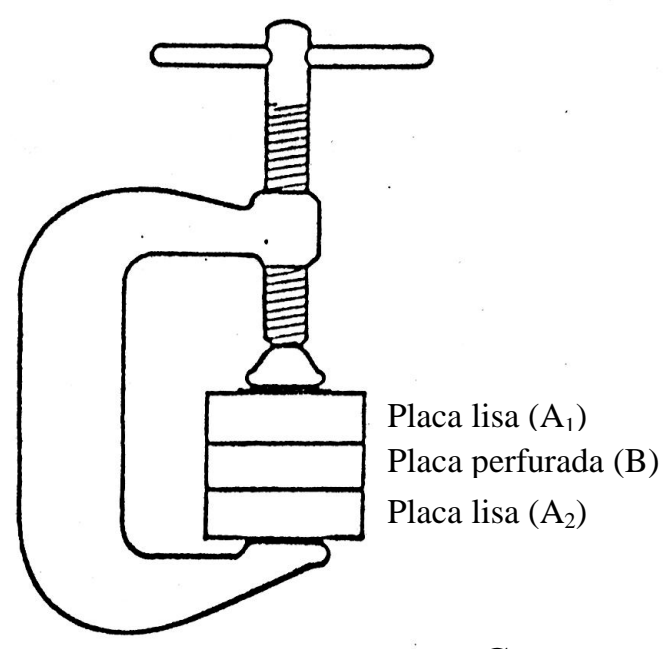

C

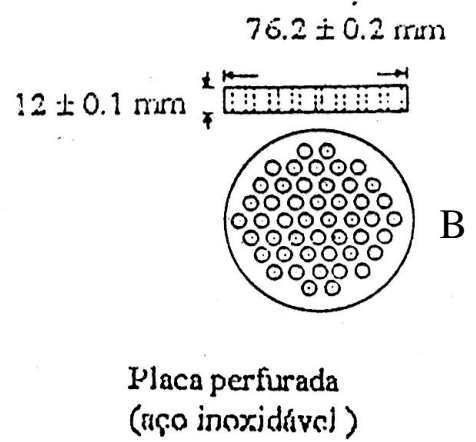

$O$ diametro dos 48 furos deve ser de $6.0: 0.1 \mathrm{mms}$.

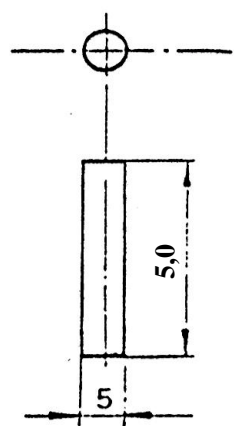

Pino removedor

$\mathrm{D}$

Flacas na rnursa

FIGURA 5. Croqui do molde para confecção de corpos de prova para o ensaio de resistência à compressão, segundo a norma ASTM F451-86. Dimensões em milímetro. 


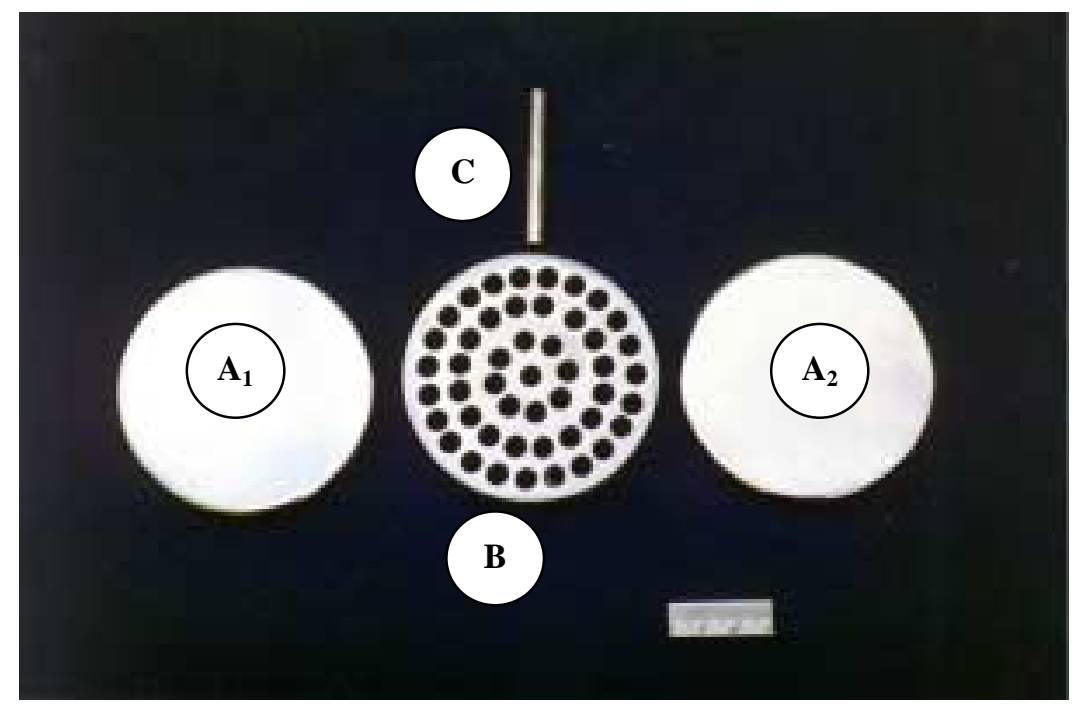

FIGURA 6. Sistema de moldagem dos CPDs. $\left(\mathrm{A}_{1}\right.$ e $\left.\mathrm{A}_{2}\right)$ Placas lisas inferior e superior, respectivamente. (B) placa perfurada. (C) Pino removedor.

\subsection{Confecção dos corpos de prova}

Após o preparo dos quatro grupos experimentais de cimento ósseo, dois grupos manuais e dois a vácuo, a massa em estado pastoso foi transferida para o conjunto de moldagem.

Inicialmente, uma das tampas lisas (base) foi posicionada sobre uma mesa, o molde colocado sobre a base, lubrificado com um desmoldante Elgin ${ }^{\circledR}$ para facilitar tanto a colocação do cimento ósseo no conjunto de moldagem, assim como a sua remoção posteriormente.

A massa foi despejada sobre o molde e, com as mãos recobertas pela luva cirúrgica, acomodava-se a massa dentro dos orifícios do molde, até que todos os orifícios estivessem adequadamente preenchidos. 
A tampa superior do molde foi então colocada, formando um bloco, o excesso de cimento ósseo entre as placas foi removido e o conjunto moldagem, pressionado em uma morsa pelo período de uma hora.

Completada uma hora, afrouxava-se a morsa, removia-se o conjunto de moldagem, separavam-se as tampas superior e inferior. Utilizando-se a granulometria, os CPDs eram regularizados, removendo-se todo o excesso de cimento ósseo tanto da parte superior como da inferior do molde, obtendo-se assim corpos de prova regulares com a altura de $12,0 \pm 0,1 \mathrm{~mm}$ e diâmetro de $6,0 \pm 0,1$ $\mathrm{mm}$.

A remoção dos CPDs foi realizada cuidadosamente com a ajuda do pino removedor . Após a remoção dos 48 CPDs, uma cuba de aço inoxidável estéril era utilizada para armazená-los nas condições ambientais pré-determinadas.

A identificação dos CPDs foi feita aleatoriamente, sendo numerados de 1 a 48. Cada grupo foi identificado de acordo com a marca do cimento ósseo e o tipo de mistura utilizado. Cada grupo experimental foi composto de 48 CPDs, perfazendo um total de 192 CPDs.

A Figura 7 apresenta exemplos de CPDs obtidos para os testes de compressão. 


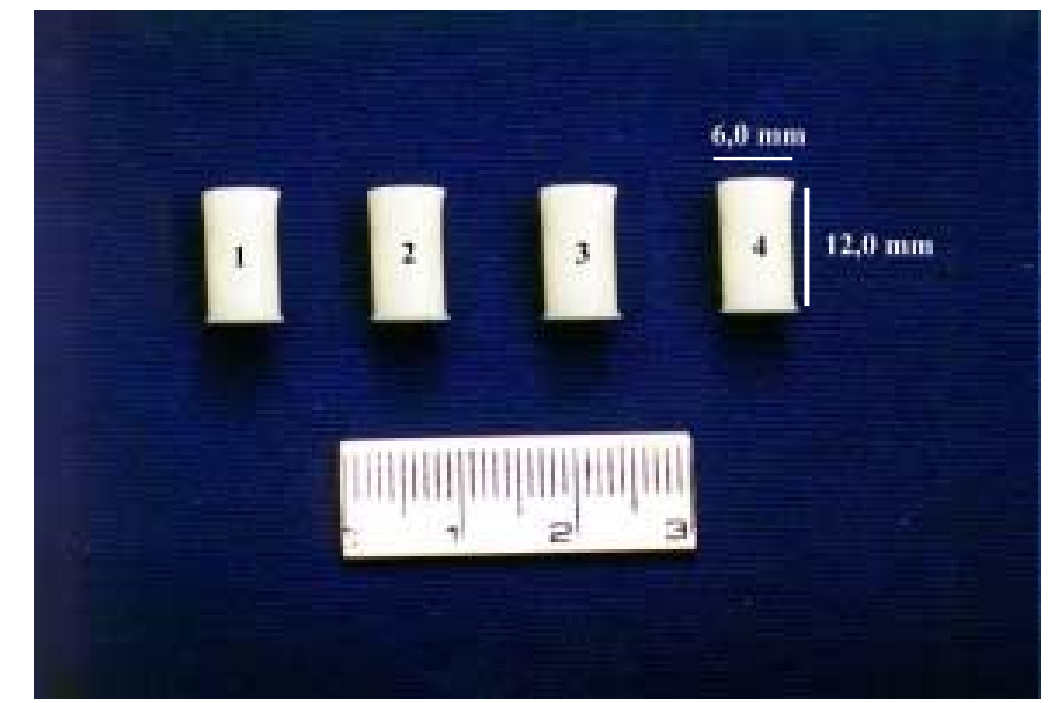

FIGURA 7. Exemplos de corpos de prova e sua de identificação.

\subsection{Exame Macroscópico}

Antes da realização dos ensaios mecânicos de compressão, foi realizada uma avaliação macroscópica em cada corpo de prova a fim de observar a existência de qualquer irregularidade grosseira que porventura pudesse estar presente.

Para cada corpo de prova foram realizadas três medidas, utilizando-se paquímetro digital Mitutoyo $^{\circledR}$ : uma medida para a altura e duas outras medidas para o diâmetro do corpo de prova.

O objetivo da inspeção macroscópica após a obtenção dos corpos de prova foi o de certificar-se de que todos eles estavam dentro dos padrões requeridos pelas normas ISO 5833 e ASTM F451-86.

Apenas $1 \mathrm{CPD}$, pertencente ao grupo $1 \mathrm{M}$, foi descartado por não apresentar as medidas padronizadas pelas normas, e, para não alterar os resultados, um corpo de prova de cada um dos outros três grupos experimentais foi descartado. 
Após a realização dos ensaios de compressão dos quatro grupos experimentais, novamente foram realizadas medidas da altura e do diâmetro dos CPDs.

As medidas obtidas antes e após os testes de compressão foram comparadas e nos permitiram calcular as deformações apresentadas por cada CPD e o cálculo da mediana para a deformação de cada grupo experimental.

\subsection{Ensaios de compressão}

Os ensaios de compressão foram realizados em máquina universal de ensaios (M.U.E.), do Laboratório de Bioengenharia da Faculdade de Medicina de Ribeirão Preto - USP. Os ensaios foram realizados $(24 \pm 2)$ h após a mistura, a uma temperatura ambiente de $21 \pm 2{ }^{\circ} \mathrm{C}$, com umidade relativa do ar de $80 \pm 10 \%$. A M.U.E. foi dotada de uma célula de carga $\operatorname{Kratos}^{\circledR}$, com capacidade de carga de 500 kgf. Foi utilizada uma pré-carga de 5 kgf e uma velocidade de aplicação de carga de $0,1 \mathrm{~mm} / \mathrm{min}$, com um tempo de acomodação de $30 \mathrm{~s}$. A deformação foi medida por um relógio comparador Mitutoyo ${ }^{\circledR}$, com precisão de $0,01 \mathrm{~mm}$. Cada teste durou em média 5 minutos, sendo os dados de cada corpo de prova, como identificação, mensuração, padronização e valores obtidos nos ensaios de compressão anotados em ficha própria (Anexo C).

A Figura 8 ilustra os detalhes da máquina universal de ensaios de compressão ao inserir um CPD do nosso experimento, mostrando a montagem do 
CPD, a célula de carga (A) e o relógio comparador (B). A Figura 9 exemplifica um CPD ao ser ensaiado, em uma imagem aproximada.

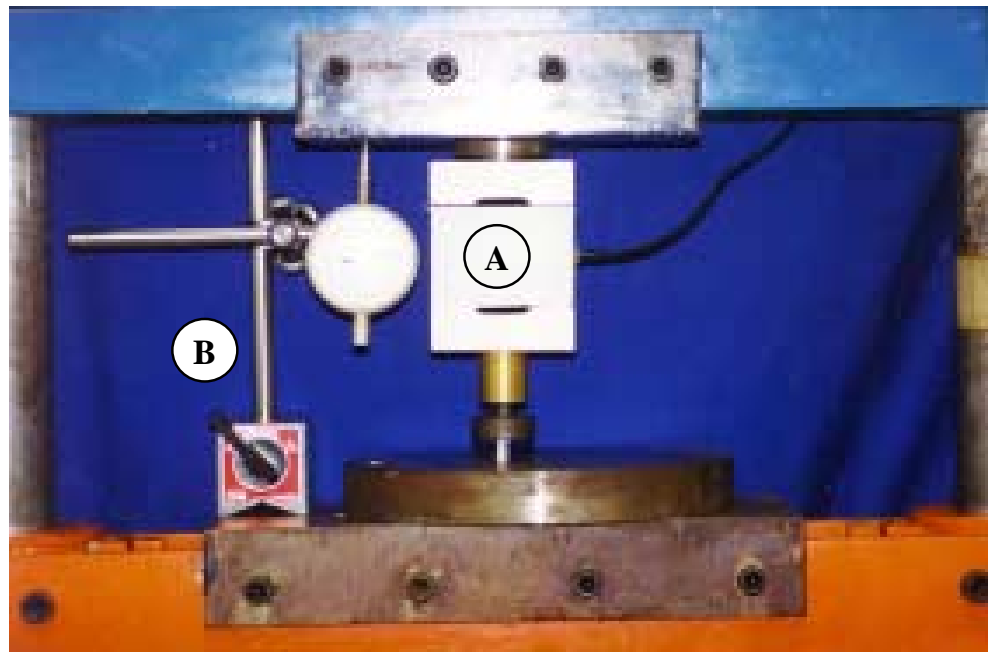

FIGURA 8. Dispositivos para os ensaios de compressão, a célula de carga (A) e o relógio comparador (B) com paralelismo entre as superfícies do corpo de prova..

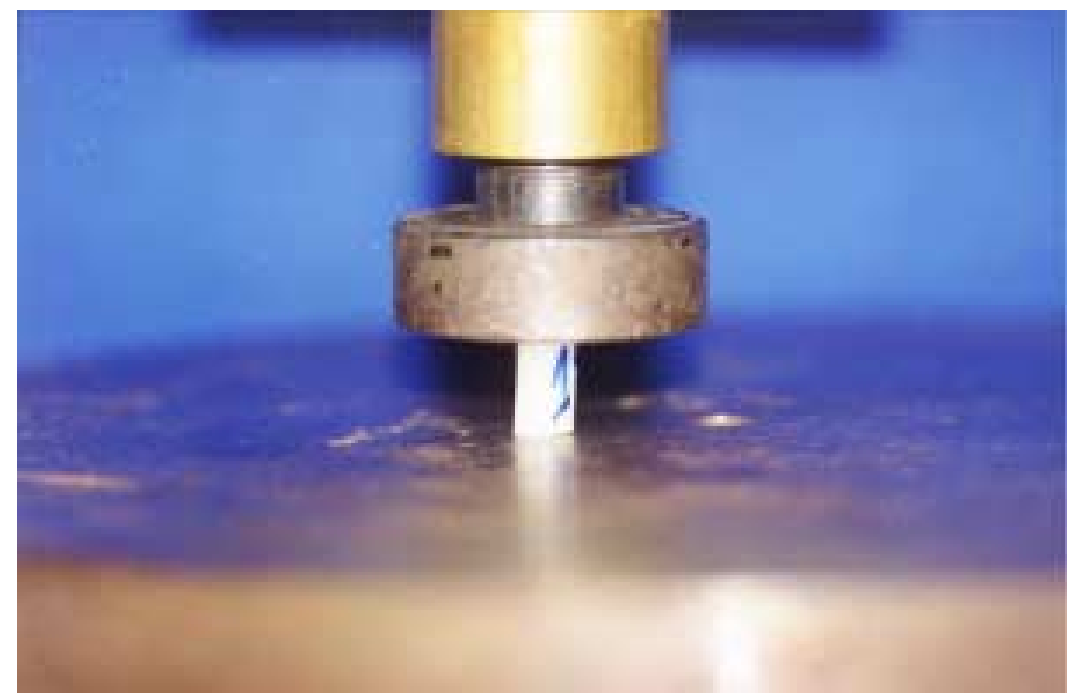

FIGURA 9. Detalhe de um CPD ao ser ensaiado.

Após a coleta desses dados, estes foram transferidos para o programa Microsoft Excel, da Microsoft ${ }^{\circledR}$, e os valores de tensão e deformação no limite de 
proporcionalidade e o módulo de elasticidade para cada corpo de prova de cada grupo experimental foram elaborados (Grupo 1M [Anexo D]; Grupo 1V [Anexo E]; Grupo 2M [Anexo F]; Grupo 2V [Anexo G]).

Com os gráficos tensão $x$ deformação (Figura 10) obtidos de cada ensaio, utilizando-se o Programa Microsoft Excel da Microsoft ${ }^{\circledR}$, foi calculada, a mediana para: tensão no limite de proporcionalidade (ponto C), deformação no limite de proporcionalidade, módulo de Young (E) para cada um dos quatro grupos experimentais de cimento ósseo.

Foram analisados os valores da tensão (MPa) e deformação (\%) no limite de proporcionalidade (C) e a tangente $\alpha$ (cateto oposto/cateto adjacente), que é o módulo de elasticidade.

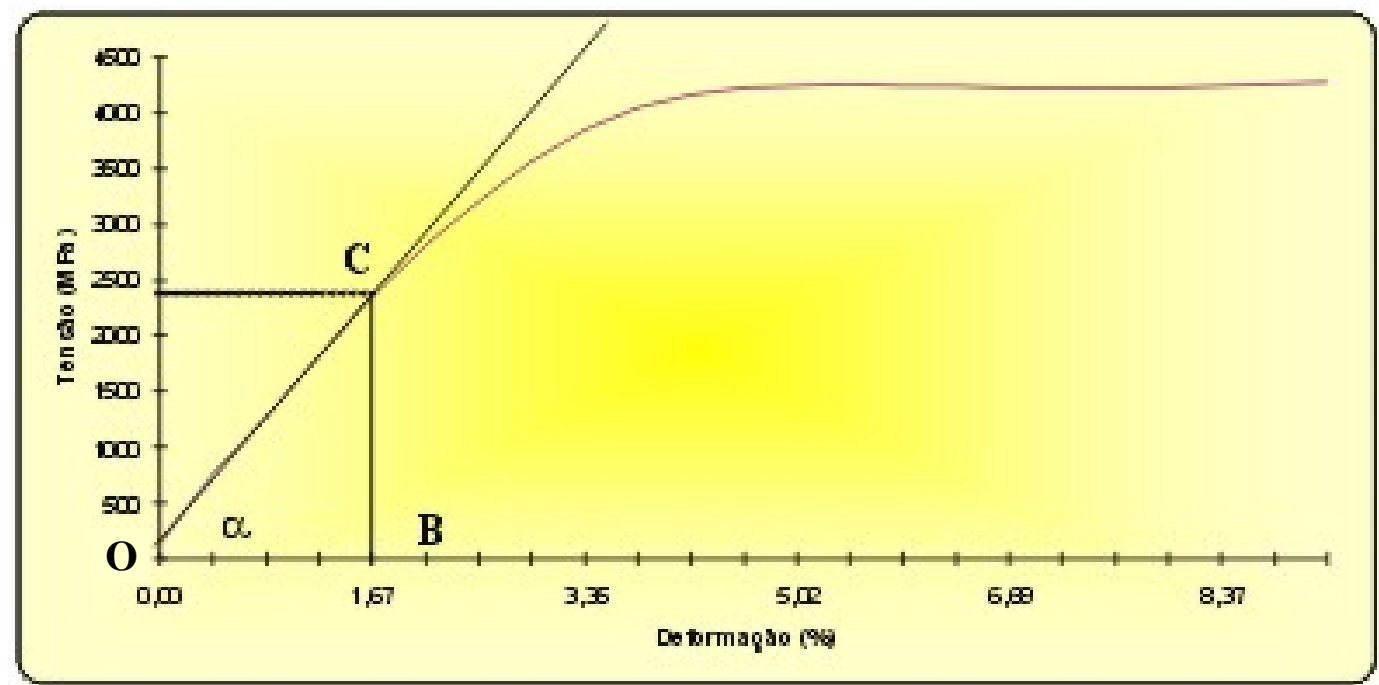

FIGURA 10. Gráfico tensão $x$ deformação, ilustrando a carga e a deformação no limite de proporcionalidade (ponto $\mathrm{C}$ ), a reta que representa a inclinação da curva (tg $\alpha)$ e o triângulo formado pela união dos pontos (OBC). 


\subsubsection{Limite de proporcionalidade}

O limite de proporcionalidade (ponto C) é identificado como sendo o último ponto onde se encontra o segmento reto da curva tensão versus deformação.

Traçou-se uma curva de regressão, seguindo a inclinação, identificando-se o último ponto inserido nesta reta. As coordenadas deste ponto representam o limite de proporcionalidade para a curva tensão versus deformação. O critério neste experimento para a identificação do limite de proporcionalidade foi que os valores obtidos para carga que aumentavam progressivamente numa velocidade rápida começassem a decrescer ou se mantivessem estacionários por alguns segundos. Após a observação de um desses dois fatos, quatro outras medidas eram ainda realizadas e somente após a realização dessas quatro outras medidas a carga era removida. Para cálculo da tensão e da deformação utilizamos medidas de deformação de 1,10 mm. Para o cálculo da deformação final a seguinte fórmula foi utilizada:

\section{comprimento inicial - comprimento final comprimento inicial}

A seguir, esses valores de deformação em $\mathrm{mm}$ foram transformados em valores percentuais, facilitando-se assim a comparação da deformação sofrida por cada corpo de prova a cada intervalo de aplicação de carga. 


\subsection{Avaliação radiográfica da porosidade}

Os corpos de prova devidamente identificados foram radiografados utilizando-se mamógrafo de alta resolução Senograph ${ }^{\circledR}$ 700T, marca GE, com magnificação 1,5x, o filme utilizado foi o $\operatorname{Kodak}^{\circledR}$ MIN R 2000 (Anexo H).

As radiografias, a seguir, foram copiadas por scanner não reflexivo, as imagens armazenadas em um Compact Disc e posteriormente inspecionadas no programa Corel PHOTOPAINT ${ }^{\circledR} 9$ para que pudéssemos observar a presença ou não das bolhas, não sendo possível quantificar o volume e a quantidade de bolhas apresentadas por cada grupo experimental, a avaliação foi subjetiva.

\subsection{Análise dos dados}

Comparamos os resultados obtidos relativos à tensão e à deformação no limite de proporcionalidade e Módulo de Young de cada grupo experimental com os demais.

Para análise de dados utilizou-se o Teste de Análise de Variância de KruskalWallis para cada grupo experimental (Anexos I, J e L), com nível de significância de 5\% ( $p \leq 0,001)$ e o método de múltipla comparação (Método de Dunn’s), que estabeleceu as seguintes comparações, com nível de significância $p \leq 0,05$ : Grupo1M 
x Grupo1V; Grupo1M x 2M; Grupo1M x Gupo2V; Grupo1V x Grupo2M; Grupo1V x Grupo 2V e Grupo 2M x Grupo 2V (Anexos I, J e L). 


\section{RESULTADOS}

\subsection{Ensaios de compressão}

O cálculo das medianas da curva tensão $x$ deformação dos quatro grupos experimentais apresentado na Figura 11, elaborado a partir de dados obtidos nos ensaios de compressão de cada um dos 47 corpos de prova (Anexos D, E, F, e G) de cada grupo experimental submetido ao teste de Análise de Variância de KruskalWallis, mostrou uma diferença estatisticamente significante quanto ao valor do módulo de elasticidade desses quatro grupos experimentais de cimento ósseo $(p \leq 0,001)$, sendo o maior valor do módulo de elasticidade encontrado no grupo $2 \mathrm{M}$ (1563 MPa). 


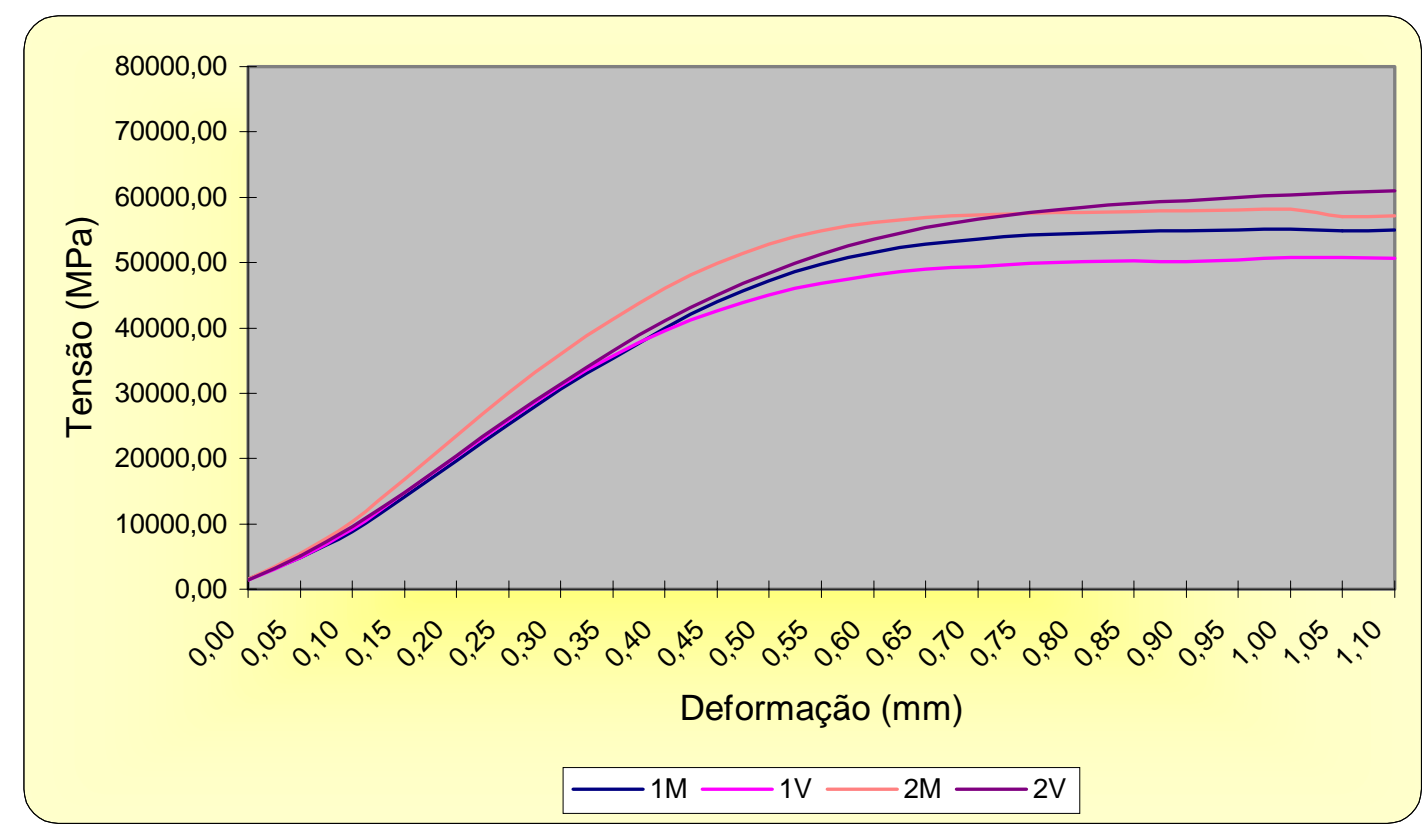

FIGURA 11 -Representação gráfica da curva tensão x deformação para os quatro grupos experimentais ensaiados.

\subsubsection{Módulo de elasticidade}

O módulo de elasticidade do cimento ósseo nacional preparado manualmente (Grupo 1M) foi de 1389,00 MPa; para o grupo preparado a vácuo (Grupo 1V) foi de 1263,00 MPa, não havendo diferença estatisticamente significativa entre esses dois grupos de cimento ósseo, quando analisados pelo Método de Dunn's $(p<0,05)$ (Anexo I).

Para os grupos de cimento ósseo importado, o preparado manualmente (Grupo 2M) com o maior valor de módulo de elasticidade 1563,00 MPa, este resultado foi estatisticamente significativa quando comparado aos grupos experimentais $1 \mathrm{M}, 1 \mathrm{~V}$ e 2V; para o grupo preparado a vácuo (Grupo 2V) o módulo 
de elasticidade foi de 1402,00 $\mathrm{MPa}$, sendo este valor maior e estatisticamente significativo quando comparado ao grupo $1 \mathrm{~V}(p<0,05)$.

A Figura 12 ilustra a mediana dos quatro grupos experimentais de cimento ósseo analisados quanto ao módulo de elasticidade, no qual observamos que o cimento ósseo importado apresentou os maiores valores de módulo de elasticidade para ambos os métodos de mistura, sendo a mistura manual a de maior valor nesta propriedade. O cimento nacional apresentou valores menores quanto ao módulo de elasticidade quando comparado ao cimento ósseo importado e a mistura manual do cimento nacional foi a que apresentou o maior módulo de elasticidade.

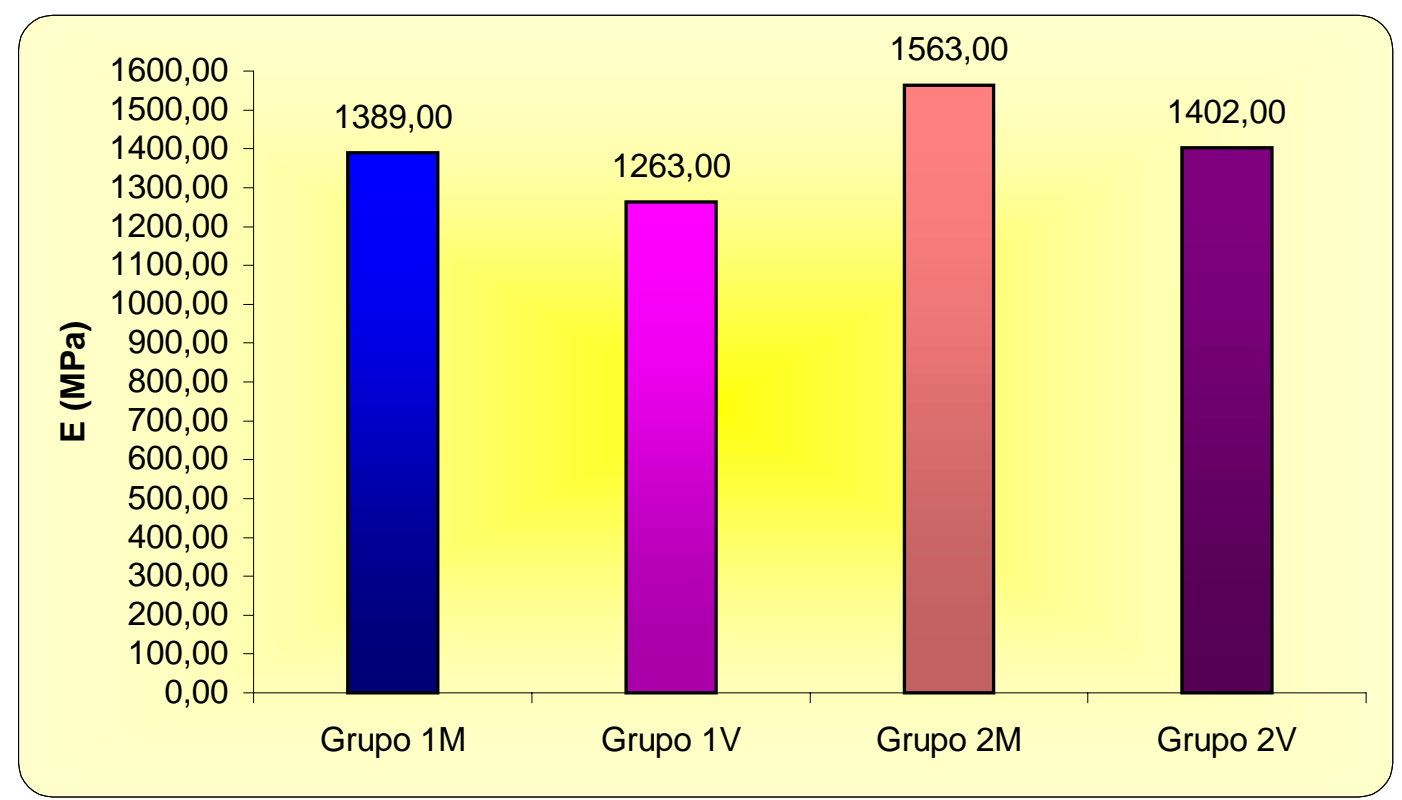

FIGURA 12 - Medianas do módulo de elasticidade dos quatro grupos experimentais. 


\subsubsection{Tensão no limite de proporcionalidade}

O teste de análise de variância de Kruskal - Wallis aplicado para avaliar a propriedade de tensão no limite de proporcionalidade dos quatro grupos experimentais de cimento ósseo (Anexo J) mostrou uma diferença significativa no cálculo das medianas da propriedade de tensão no limite de proporcionalidade $(p<$ $0,001)$

Os valores medianos da tensão no limite de proporcionalidade sendo que para o grupo $1 \mathrm{M}$ esse valor foi de $39,40 \mathrm{MPa}$, resultado maior e estatisticamente significativo quando comparado com os grupos $1 \mathrm{~V}$ e $2 \mathrm{M}(p<0,05)$. O grupo $1 \mathrm{~V}$, com um valor menor de tensão no limite de proporcionalidade $36,15 \mathrm{MPa}$ em relação aos grupos $1 \mathrm{M}$ e $2 \mathrm{~V}$, apresentou diferença estatisticamente significativa em relação a esses grupos $(p<0,05)($ Anexo $\mathrm{J})$.

Para os grupos de cimento ósseo importado, preparados manualmente e a vácuo, o cálculo da mediana para a tensão no limite de proporcionalidade foi de 35,80 MPa e 39,65 MPa, respectivamente, o grupo 2M foi o que apresentou o menor valor de tensão no limite de proporcionalidade; a diferença foi estatisticamente significativa quando comparada aos grupos experimentais $1 \mathrm{~V}$ e $2 \mathrm{~V} \quad(p<0,05)$ (Anexo J).

A Figura 13 visualiza os dados de tensão no limite de proporcionalidade dos quatro grupos experimentais, os maiores valores foram apresentados pelo grupo de cimento nacional preparado manualmente (Grupo 1M) e pelo grupo de cimento importado preparado a vácuo (Grupo 2V). 


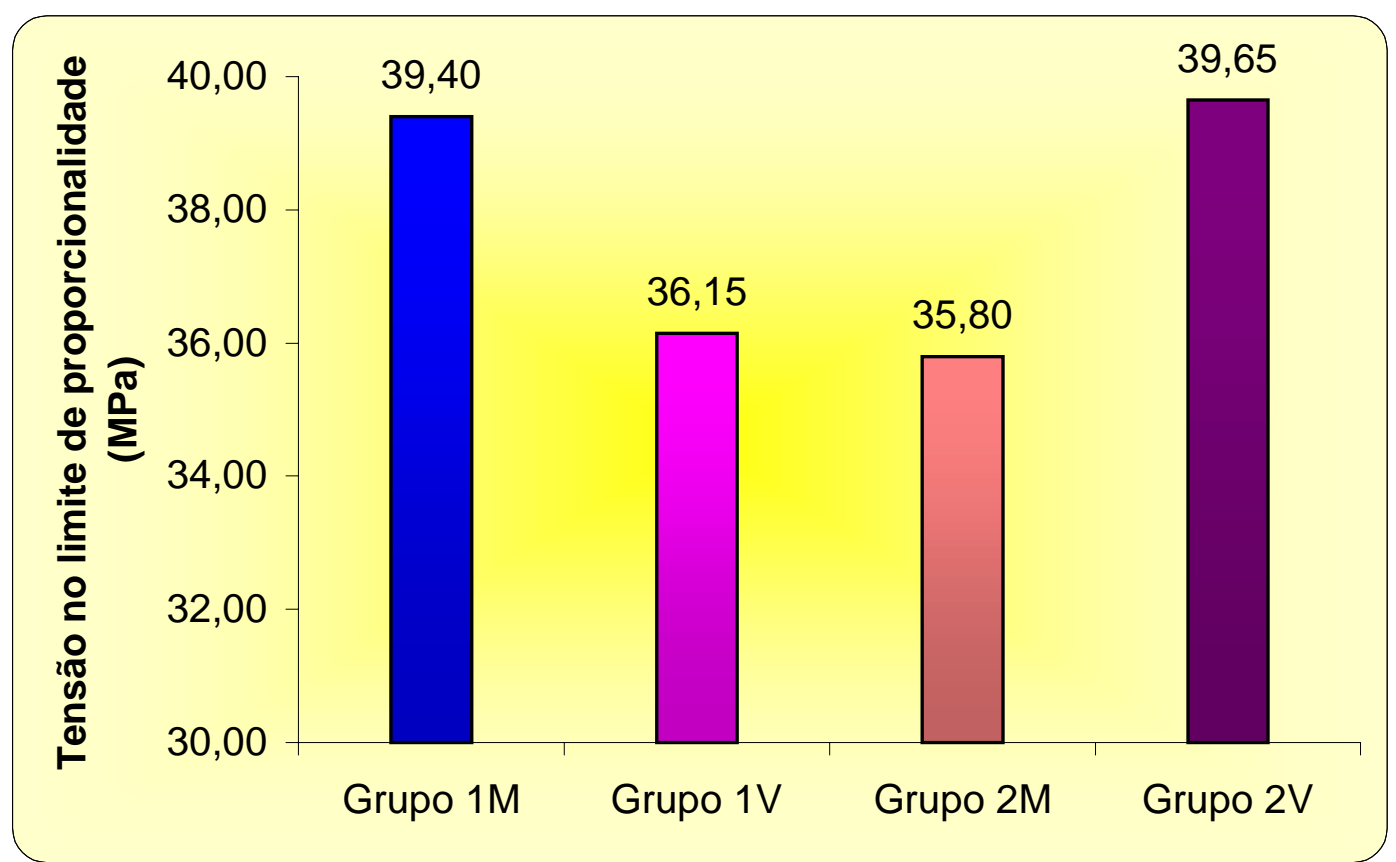

FIGURA 13 - Valores das medianas da tensão no limite de proporcionalidade dos quatro grupos experimentais de cimento ósseo.

\subsubsection{Deformação no limite de proporcionalidade}

A porcentagem de deformação no limite de proporcionalidade entre os quatro grupos experimentais apresentou diferença estatisticamente significante $(p<0,001)$ (Anexo L); a maior deformação foi encontrada no grupo 1M.

O cálculo da mediana do grupo $1 \mathrm{M}$ foi de $3,36 \%$, este resultado foi maior e estatisticamente significativo quando comparado ao grupo $2 \mathrm{M}$. Para o grupo $1 \mathrm{~V}$, a mediana da porcentagem de deformação no limite de proporcionalidade foi de $2,95 \%$, valor maior e estatisticamente significativo em comparação ao grupo $2 \mathrm{M}$.

O grupo experimental $2 \mathrm{M}$ com uma deformação de $2,53 \%$, apresentou valor menor e estatisticamente significante quando comparado aos grupos $1 \mathrm{M}$ e $1 \mathrm{~V}$, e o 
grupo 2V, com uma deformação de 2,93\%, apresentou valor maior e estatisticamente significativo em comparação ao grupo experimental $2 \mathrm{M}(p<0,05)$ (Anexo L).

A Figura 14 demonstra que os maiores valores percentuais de deformação foram encontrados nos grupos $1 \mathrm{M}$, ou seja, cimento nacional preparado manualmente, seguido pelo grupo $1 \mathrm{~V}$, cimento nacional preparado a vácuo. Para os cimentos importados, o grupo preparado a vácuo foi o que apresentou o maior valor percentual de deformação, e o preparado manualmente foi o de menor valor percentual de deformação, se comparado ao cimento de mesma nacionalidade ou ao cimento nacional.

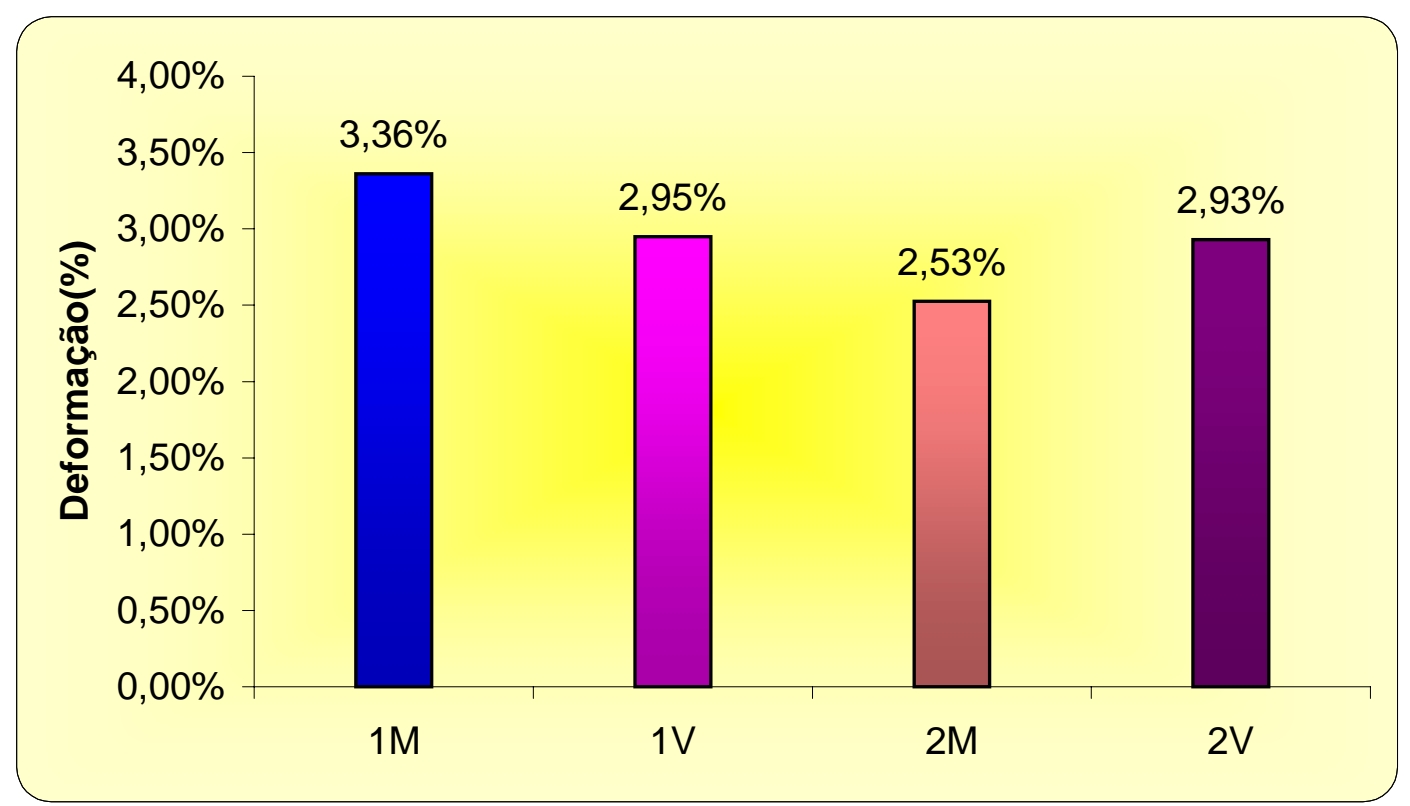

FIGURA 14 - Gráfico representativo das medianas das porcentagens de deformação dos quatro grupos experimentais de cimento ósseo. 


\section{DISCUSSÃ̃}

Existe uma extensa literatura sobre as propriedades mecânicas do cimento ósseo acrílico importado consideradas importantes para a sua caracterização como biomaterial de uso abrangente por todo o mundo, para especificamente fixar os componentes protéticos nas cirurgias de substituição articular especialmente do quadril, do joelho e do ombro.

A produção industrial do cimento ósseo acrílico requer tecnologia avançada, desenvolvida apenas em poucos países do primeiro mundo. Sua formulação química exata é considerada segredo industrial pelas indústrias que o produzem, que apenas fornecem os componentes empregados na sua fabricação. Isto faz com que o custo do uso do cimento acrílico importado seja elevado, adicionando-se ainda a seu preço os valores necessários para sua importação e comercialização.

O cimento chamado de nacional é na realidade também o cimento acrílico importado, uma vez que não temos tecnologia para produzi-lo. No entanto, o fato de ser embalado e comercializado por empresa nacional torna seu custo mais barato, acessível aos pacientes assistidos pelo Sistema Único de Saúde. 
Existe um certo desconforto por parte dos ortopedistas brasileiros quanto ao uso deste cimento nacionalizado, temerosos de que o baixo custo seja obtido graças à perda de qualidade de um produto a ser implantado em seus pacientes.

A diferença existente é que os componentes nacionalizados são dosados mecanicamente enquanto os componentes do cimento importado são dosados eletronicamente.

Sabe-se que todas as marcas comerciais de cimento ósseo estão sujeitas a uma série de variáveis que podem interferir em suas propriedades mecânicas, como exemplos citamos as formulações comerciais, as condições de estocagem, os métodos de mistura entre outros.

Os métodos de preparação tão discutidos a partir do desenvolvimento das técnicas de cimentação de $2^{\mathrm{a}}$ geração (WIXSON, LAUTENSCHLAGER, 1987; LIDGREN, BODELIND \& MÖLLER, 1987; WANG et al., 1996; CHAN, AHMED \& JOHNSON in MORREY , 1996; KURDY, HODGKINSON \& HAYNES, 1996; FRISTCH, 1996; LEWIS, 1997; GEIGER et al., 2001) propõem uma melhora da resistência mecânica dos cimentos ósseos como conseqüência da redução da porosidade do cimento de 5 a 10\%, valores considerados normais, para a mistura manual (CHAN, AHMED \& JOHNSON in MORREY , 1996) para menos de $1 \%$ para a mistura a vácuo (WIXSON, LAUTENSCHLAGER \& NOVAK, 1987). Porém, estes dados não puderam ser confirmados neste trabalho pela dificuldade em dimensionar-se e quantificar-se a porosidade apresentada por cada corpo de prova de cada um dos quatro grupos experimentais do cimento ósseo, de maneira precisa como proposto por JAMES et al. (1992), que investigaram a porosidade utilizando a fractomografia, que permitiu dimensionar e quantificar o 
número e o tamanho das macro e microporosidades. Nossa análise foi somente visual, utilizando-se uma imagem ampliada na tela do computador, constatando-se que o cimento importado misturado a vácuo apresentou maior porosidade que os outros três grupos experimentais: nacional e importado preparados manualmente e nacional preparado a vácuo, com níveis de porosidade menor para os grupos que foram preparados manualmente, mesmo que em níveis talvez acima do que a literatura considera como normais.

Na tentativa de justificar esse fato, dois novos grupos de cimento importado foram preparados nas mesmas condições descritas anteriormente e pôde-se observar que a porosidade foi realmente elevada.

Alguns fatores foram analisados na tentativa de justificar esses dados. Primeiramente, que nem todas as formulações comerciais de cimento ósseo teriam seus níveis de porosidade diminuídos pela mistura a vácuo (WANG et al., 1996); em seguida que nem todos os equipamentos utilizados para a mistura a vácuo diminuem a porosidade do cimento ósseo, explicado pelo fato de que estes sistemas, em muitas situações, não misturam adequadamente o componente em pó, que fica concentrado dentro do misturador e não completamente misturado ao componente líquido (KURDY, HODGKINSON \& HAYNES, 1996).

Um terceiro fato considerado extremamente importante para a mistura a vácuo foi a pressão atmosférica, questionada durante toda a análise deste trabalho. A literatura propõe que a utilização de sistemas de vácuo (WIXSON, LAUTENSCHLAGER \& NOVAK, 1987; CHAN, AHMED \& JOHNSON in MORREY , 1996; KURDY, HODGKINSON \& HAYBES, 1996) deve proporcionar uma pressão atmosférica considerada ideal em torno de $500-550 \mathrm{mmHg}$, sendo que 
pressões atmosféricas que variam entre $200-400 \mathrm{mmHg}$ e maiores que $600 \mathrm{mmHg}$ são incapazes de diminuir os percentuais de porosidade e até mesmo introduzindo mais porosidade na mistura, como em situações em que a pressão atmosférica é maior que $600 \mathrm{mmHg}$, facilitando a evaporação do monômero (GEIGER et al., 2001).

WIXSON, LAUTENSCHLAGER \& NOVAK (1987), ao testarem o cimento ósseo acrílico Simplex-P a uma pressão de vácuo de 550mmHg, encontraram níveis de porosidade menores que $1 \%$ quando comparados à mistura manual. Os testes de compressão mostraram uma resistência à compressão $24 \%$ maior para o grupo misturado a vácuo e $12 \%$ para os centrifugados ao serem comparados à mistura manual.

LING \& LEE (1998) concluem que a mistura a vácuo reduz a porosidade em 2\% a 5\% comparados à mistura manual, porém, há uma predisposição de $30 \%$ maior para revisões tardias e $12 \%$ para as falhas do implante, "associando o insucesso do método de mistura aos sistemas de vácuo disponíveis no mercado".

A pressão atmosférica empregada neste trabalho esteve em torno de 300-350 mmHg, situação disponível na maioria das salas cirúrgicas brasileiras na preparação a vácuo do cimento ósseo.

O fato de a porosidade não ter sido diminuída pela mistura a vácuo no grupo de cimento nacional é justificada, talvez, por trabalhos como de HANSEN \& JANSEN (1992) e LING \& LEE (1998), que encontraram dados semelhantes em seus trabalhos e concluíram que nem todas as formulações de cimento ósseo têm a sua resistência mecânica aumentada pela mistura a vácuo, citando exemplos de formulações comerciais de cimentos ósseos testados à compressão que apresentavam 
uma resistência maior igual $100 \mathrm{MPa}$ quando misturados manualmente e de 91 a 95 MPa quando misturados a vácuo.

O grupo de cimento importado misturado a vácuo teve a sua resistência mecânica à compressão melhorada quando comparado à mistura manual do mesmo grupo condição relatada por inúmeros trabalhos (CHAN, AHMED \& JOHNSON, 1996; LEWIS, 1997; FRISTCH, 1996; LIDGRE, BODELIND \& MÖLLER, 1987, WANG et al., 1993; WIXSON, LAUTENSCHLAGER \& NOVAK, 1987), que utilizaram esta mesma formulação comercial. O cimento ósseo é naturalmente frágil, a sua resistência à compressão é maior que sua resistência à tensão e varia usualmente entre 64 a $103 \mathrm{MPa}$, representando 50-70\% da resistência do osso cortical humano (SAHA \& PAL, 1984; CHAN, AHMED \& JOHNSON in MORREY , 1996).

Quanto ao módulo de elasticidade, ou seja, a medida da rigidez dos quatro grupos experimentais, para o cimento nacional preparado manualmente e a vácuo, o grupo mais rígido foi o preparado manualmente; fato semelhante ocorreu nos grupos de cimento importado. Ao se estabelecer a relação entre cimento nacional e importado, este último foi o que apresentou maior módulo de elasticidade.

Os valores de módulo de elasticidade variam entre 2140-3100 MN/m² (SAHA \& PAL, 1984), com uma média de 2700 MPa (CHAN, AHMED \& JOHNSON in MORREY, 1996), representa cerca de $15 \%$ do coeficiente de elasticidade do osso cortical. Os valores aproximados do módulo de elasticidade do cimento, do osso e dos metais ortopédicos são 2 para o polimetilmetacrilato (PMMA), 20 para o osso, 100 para o titânio e 200 para o aço inoxidável e liga de cobalto (HARKESS in CANALE, 1998) O cimento deve funcionar como um 
absorvedor de carga e distribuí-la nas interfaces cimento/osso e cimento/prótese. As causas de afrouxamento asséptico podem originar-se de falhas nas interfaces cimento/osso, entre implante/osso ou no próprio manto de cimento (WANG et al., 1996). A propriedade da viscoelasticidade do cimento ósseo pode representar o sucesso ou a falha da cimentação nas substituições articulares; este fato ainda não é muito bem entendido, podendo a viscosidade natural contribuir para o afrouxamento (NORMAN et al., 1995). Teoricamente, um módulo de elasticidade menor é vantagem nas hastes do quadril sem cimento, porque reduz o estresse do componente com o osso, sendo possível diminuir a remodelação óssea causada pelo estresse. Por outro lado, para os componentes cimentados, um maior módulo de elasticidade (maior rigidez) é uma vantagem, porque reduz o estresse ao redor do manto de cimento do componente e diminui o risco de falha do cimento, mas isso é uma desvantagem porque pode levar à reabsorção óssea, resultando eventualmente num suporte ruim para o cimento e com conseqüente falha deste, produzindo afrouxamento do componente. Claramente, o módulo de elasticidade pode ser considerado em contexto com os outros materiais utilizados nas artroplastias e seus módulos e não justifica isolado de cada um dos componentes utilizados nas cirurgias de substituição (HARKESS in CANALE, 1998).

Os índices de afrouxamento femoral que diminuíram marcadamente para menos de 3\%, em um seguimento de 10 anos (GEIGER et al., 2001), estão associados a alterações como o desenvolvimento de novas ligas para a fabricação de implantes, novos desenhos protéticos e as modernas técnicas de cimentação que procuram produzir um manto de cimento ósseo homogêneo que se inicia com uma adequada preparação e lavagem do canal femoral para a remoção dos debris de 
tecidos, injeção retrógrada do cimento, tamponamento do canal femoral, a pressurização do cimento ósseo dentro do canal, permitindo sua maior interdigitação com o osso, além do uso de centralizadores que posicionam a haste femoral no centro do canal, possibilitando a formação de um manto de cimento ósseo uniforme.

Os valores de módulo de elasticidade inferiores apresentados por este trabalho, quando comparado à literatura, provavelmente estão relacionados ao fato de que a velocidade de carga aplicada durante a execução dos testes de resistência mecânica à compressão, $0,1 \mathrm{~mm} / \mathrm{min}$, utilizando a Máquina Universal de ensaios, foi inferior à velocidade padronizada pela ISO 5833, $20 \mathrm{~mm} / \mathrm{min}$, pois era a velocidade que o equipamento disponível em nosso laboratório permitia no momento da realização dos testes mecânicos.

A comparação entre os diferentes tipos de cimento misturados diferentemente, teria importância fundamental se demonstrasse claramente a superioridade do cimento importado misturado a vácuo sobre os demais. No entanto, o cimento importado misturado manualmente foi o que apresentou maior módulo de elasticidade com diferença estatisticamente significante em relação aos outros. Podemos justificar este achado considerando que nossa técnica de mistura a vácuo não funcionou como sugerido pela nossa análise da porosidade, ou que realmente a mistura a vácuo não é eficaz.

É certo que a mistura do cimento importado a vácuo exibiu maior tensão no limite de proporcionalidade, indicando a capacidade maior deste grupo em absorver carga sem deformação permanente. Entretanto, o valor obtido neste ensaio para o cimento importado misturado a vácuo não foi estatisticamente diferente do valor obtido para a mistura manual do cimento nacional. Isto mostra não haver diferença 
entre o cimento importado e o nacionalizado e, novamente, que nossa técnica de mistura a vácuo não foi eficiente.

A deformação no limite de proporcionalidade foi menor no grupo do cimento importado misturado manualmente, sendo esta diferença estatisticamente significante em relação aos outros grupos. Deve ser lembrado que nos testes mecânicos de compressão a deformação elástica sob carga tende a aproximar as moléculas do corpo de prova, e sua resistência à carga pode ser mantida pelo menos no início da rotura do corpo testado. Deve ser ressaltado que nossos testes não produziram fraturas macroscópicas nos corpos de prova. A importância da menor deformação neste grupo reside no fato de ter sido ele o que apresentou o maior módulo de elasticidade e, uma vez que também apresentou a menor tensão no limite de proporcionalidade, o seu módulo de elasticidade elevado é devido principalmente a sua baixa deformação.

A preparação a vácuo não diminuiu a deformação percentual no limite de proporcionalidade para o cimento importado nem para o cimento nacional.

Nossos resultados parecem indicar que a mistura a vácuo, da forma que realizamos, não teve qualquer efeito sobre a resistência à compressão dos nossos diferentes corpos de prova. Também não conseguimos claramente evidenciar vantagens do cimento importado sobre o cimento nacionalizado, no tocante à resistência à compressão. É importante considerarmos as diferenças entre grupos tendo-se em conta que o coeficiente de elasticidade do cimento ósseo é menos de cerca de $15 \%$ do coeficiente do osso e portanto as pequenas diferenças que observamos provavelmente não tem qualquer importância clínica. 
Neste trabalho apenas comparamos a resistência a compressão entre os diferentes cimento, e portanto não devemos extrapolar nossos resultados para outros testes mecânicos. 


\section{CONCLUSÃO}

Os testes de resistência à compressão do cimento ósseo nacionalizado e importado, preparados manualmente e a vácuo, mostraram não haver diferença importante entre os dois tipos de cimento nem entre as duas formas de misturá-los. 


\section{REFERÊNCIAS BIBLIOGRÁFICAS}

ASKEW, M.J.; KUFEL, M.F.; FLEISSNER JR, P.R.; GRADISAR JR, I.A.; SALSTROM, S.; TAN, J.S. Journal of Biomedichals Materials Research. v. 24, p. 573-580, 1990

ASTM - F 451-86.. Standard Specifications for Acrylic Bone Cement. New York, p. 97-103, sep. 1986.

BARB, W.; PARK, J.B.; KENNER, G.H.; RECUM, A.F. Intramedullary fixation of artificial hip joints with bone cement-precoated implants. I. Interfacial strengths. Journal of Biomedical Materials Research. v. 16, p. 447-458, 1982.

BISHOP, N.E.; FERGUSON, S.; TEPIC, S. Porosity reduction in bone cement at the cement-stem interface. The Journal of Bone and Joint Surgery. v. 78-B, n. 3, p. 349-356, may. 1996.

BURKE, D.W.; GATES, E.J.; HARRIS, W.H. Centrifugation as a method of improving tensile and fatigue properties of acrylic bone cement. J. Bone Joint Surgery. v. 66-A, n. 8, p. 1265-1273, oct. 1984.

CHAN, K.; AHMED, A.M.; JOHNSON, J.A. Polymethylmethacrylate. In: MORREY, B.F. (ed.) Reconstructive surgery of the joints. 2.ed. [s.l.]: [s.n.], 1996.

CHARNLEY, J. Anchorage of the femoral head prosthesis to the shaft of the femur. The Journal of Bone and Joint Surgery. V. 42B, n. 1, p. 28-30, feb. 1960.

COMBS, S.P.; GREENWALD, S. The effects of barium sulfate on the polymerization temperature and shear strength of surgical Simplex P. Clinical Orthopaedics and Related Research. n. 145, p. 287-299, nov./dec. 1979. 
DANIELS, A. U.; TOOMS, R.E.; HARKESS, J.W. Introduction and overview. In: CANALE, Terry (ed.) Campbel's operative orthopaedics. 9. ed. St. Louis, Missouri: Mosby-Year Book, 1998. v. 1. p. 212-227.

DAVIES, J.P.; BURKE, D.W.; O'CONNOR, D.O.; HARRIS, W.H. Comparison of the fatigue characteristics of centrifuged and uncentrifuged Simplex P bone cement. Journal of Orthopaedic Research . n. 5, p. 366-371, 1987.

DAVIES, J.P.; O'CONNOR, D.O.; BURKE, D.W.; JASTY, M.; HARRIS, W.H. The effect of centrifugation on the fatigue life of bone cement in the presence of surface irregularities. Clinical Orthopaedics and Related Research. n. 229, p. 156161, mar. 1988.

DEB, S.; VASQUEZ, B.; BONFIELD, W. Effect of crosslinking agents on acrylic bone cements based on poly(methylmethacrylate. 1997.

FRITSCH, E.W. Static and fatigue properties of two new low-viscosity PMMA bone cements improved by vacuum mixing. Journal of Biochemical Materials Research. v. 31, p. 451-456, 1996.

GEIGER, M.H.; KEATING, E.M.; RITTER, M.A.; GINTHER, J.A.; FARIS, P.M.; MEDING, J.B. The clinical significance of vacuum mixing bone cement. Clinical orthopaedics and related research. n. 382, p. 258-266, jan. 2001.

GOODMAN, S.B.; FORNASIER, V.L.; KEI, J. The effect of bulk versus particulate polymethylmethacrylate on bone. Clinical Orthopaedics and Related Research. $\mathrm{n}$. 232, p. 255-262, jul. 1988.

HAAS,S.S.; DICKSON, G.; BRAUER, G.M. A proposed specification for acrylic bone cement. J. Biomed. Mater. Res. Symposium. n. 6, p. 105-117, 1975.

HANSEN, D.; JENSEN, J.S. Mixing does not improve mechanical properties or all bone cements. Acta Orthop Scand. V. 63, n. 1, p. 13-18, 1992.

HARKESS, James W. Arthroplasty of hip. In: CANALE, Terry (ed.) Campbel's operative orthopaedics. 9. ed. St. Louis, Missouri: Mosby-Year Book, 1998. v. 1. p. $300-472$.

INTERNATIONAL STANDARD. ISO 5833. Implants for surgery - Acrylic resin cements. Genève: International Organization for Standardization. p. 8-17, 1992.

JAMES, S.P.; JASTY, M.; DAVIES, J.; PEIHLER, H.; HARRIS, W.H. A fractographic investigation of PMMA bone cement focusing on the relationship between porosity reduction and increased fatigue life. Journal of Biomedichals Materials Research. v. 26, p. 651-662, 1992. 
JOHNSON, J.A.; PROVAN, J.W.; KRYGIER, J.J.; CHAN, K.H.; MILLER, J. Fatigue of acrylic bone cement - effect of frequency and environment. Journal of Biomedical Materials Research. v. 23, p. 819-831, 1989.

KRAUSE, W.; MATHIS, R.S. Fatigue properties of acrylic bone cements: review of the literature. J. Biomed. Mater. Res. Applied Biomaterials. v. 22, n. A1, p. 37-53, 1988.

KRAUSE, W.; MATHIS, R.S.; GRIMES, L.W. Fatigue properties of acrylic cement: S-N, P-N, and P-S-N data. J. Biomed. Mater. Res.: Applied Biomaterials. v. 22, n. A3, p. 221-244, 1988.

KRAUSE, W.R.; GRIMES, L.W.; MATHIS, R.S. Fatigue testing of acrylic bone cements: statistical concepts and proposed test methodology. J. Biomed. Mater. Res.: Applied Biomaterials. v. 22, n. A2, p. 179-190, 1988.

KURDY, N.M.; HODGKINSON, J.P.; HAYNES, R. Acrylic bone-cement. The Journal of Arthroplasty. v. 11, n. 7, p. 813-819, 1996.

LEE, A.J.C.; LING, R.S.M.; WRIGHTON, J.D. Some properties of polymethylmethacrylate with reference to its use in orthopaedic surgery. Clinical Orthopaedics and Related Research. n. 95, sep. 1973.

LEWIS, G. Properties or acrylic bone cement: state of the art review. J. Biomed. Res. v. 38, n. 2, p. 155-182, 1997.

LEWIS, G. Effect of mixing method and storage temperature of cement constituents on the fatigue and porosity of acrylic bone cement. p. 143-149, 1999.

LEWIS, G. NYMAN, J. TRIEU, H.H. The apparent fracture toughness of acrylic bone cement: effect of three variables. Biomaterials. n. 19, p. 961-967, 1998.

LEWIS, G.; NYMAN, J.S. Toward standardization of methods of determination of fracture properties of acrylic bone cement and statistical analysis of test results. $J$. Biomed. Mater. Res. v. 53, n. 6, p. 748-768, 2000.

LIDGREN, L.; BODELIND, B.; MÖLLER, J. Bone cement improved by vacuum mixing and chilling. Acta Orthop. Scand. n. 57, p. 27-32, 1987.

LING, R.S.M.; LEE, A.J.C. Porosity reduction in acrylic cement is clinically irrelevant. Clinical Orthopaedics and Related Research. n. 355, p. 249-253, 1998.

MAJKOWSKI, R.S.; MILES, A.W.; BANNISTER, G.C.; PERKINS, J.; TAYLOR, G.J.S. Bone surface preparation in cemented joint replacement. The Journal of Bone and Joint Surgery. v. 75-B, n. 3, p. 459-463, may. 1993 
MOLINO, L.N.; TOPOLESKI, L.D.T. Effect of $\mathrm{BaSO}_{4}$ on the fatigue crack propagation rate of PMMA bone cement. 1996.

MULROY JR., R.D.; HARRIS, W.H. The effect of improved cementing techniques on component loosening in total hip replacement. The Journal of Bone and Joint Surgery. v. 72-B, n. 5, p. 757-760, sep. 1990.

MULROY, W.F.; ESTOK, D.M.; HARRIS, W.H. Total hip arthrosplasty use of socalled second-generations cementing techniques. The Journal of Bone and Joint Surgery. v. 77A, n.12, p. 1845-1852, dec. 1995.

NORMAN, T.L.; KISH, V.; BLAHA, J.D.; GRUEN, T.A.; HOSTOSKY, K. Creep characteristics of hand- an vacuum-mixed acrylic bone cement at elevated stress levels. Journal of Biomedical Materials Research. v. 29, p. 495-501, 1995.

RIMNAC, C.M.; WRIGHT, T.M.; McGILL, D.L. The effect of centrifugation on the fracture properties of acrylic bone cements. The Journal of Bone and Joint Surgery. v. 68 A, n. 2, p. 281-287, feb. 1986.

ROSTOKER, W.; LEREIM, P.; GALANTE, J.O. Effect of an in vivo environment on the strength of bone cement. Journal of Biomedical Materials Research. v. 13, p. 365-370, 1979.

SAHA, S.; PAL, S. Mechanical properties of bone cement: a review. Journal of Biomedical Materials Research. v. 18, p. 435-462, 1984.

SCHREURS, B.; SPIERINGS, P.T.J.; HUISKES, R.; SIOOFF, T.J.J.H. Effects of preparation techniques on the porosity of acrylic cements. Acta Orthop Scand. v. 59, n. 4, p. 403-409, 1988.

SCHURMANN, D.J.; BLOCH, D.A.; SEGAL, M.R.; TANNER, C.M. Conventional cemented total hip arthroplasty. Clinical Orthopaedics and Related Research. n. 240, p. 173-180, 1989.

UNIVERSIDADE DE SÃO PAULO. Faculdade de Medicina. Instituto de Ortopedia e Traumatologia - Hospital das Clinicas. Avaliação da qualidade dos cimentos ósseos encontrados no mercado nacional. Laboratório de Biomecânica LIM-41. São Paulo, 1996. 133 p. (Apostila).

WANG, J.S.; TOKSING-LARSEN, S. MÜLLER-WILLE, P.; FRAZÉN, H. is there any difference between vacuum mixing systems in reducing bone cement porosity? Journal of Biomedical Material Research (Applied Biomaterials). v. 33, p. 115-119, 1996. 
WIXSON, R.L.; LAUTENSCHLAGER, E.P.; NOVAK, M.A. Vacuum mixing of acrylic bone cement. The Journal of Arthroplasty. v. 2, n. 2, p.141-149, jun. 1987. 
ANEXO A

ASTM F451-86 


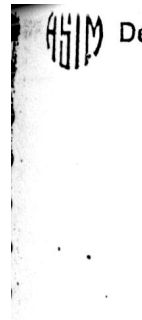

1. Scope

1.1 This specification covers sclf-curing resins used primarily for the fixation of internal orthopedic prostheses. The mixture may be used in either the predough or dough stage in accordance with the manufacturer's recommendations.

1.2 Units of premeasured powder and liquid are supplice in a form suitable for mixing, which then sets in placc.

1.3 Whilc a varicty of copolymers and comonomers may be incorporated, the composition of the set cement shall contain poly(methacrylic acid csters) as its main ingredient. 1.4 This specification covers compositional and physical performanec and packaging requirements, but does not cover toxicity nor biocompatibility of the cement. The biocompatability of acrylic bone cement has been reported in the litcrature (1)..$^{2}$

1.5 This standard may involve hazardous materials, operations, and equipment. This standard does not purport to address all of the safety problems associated with its use. It is the responsibility of the user of this soundard to cestablisth appropriate safety and health practices and determine the applicability of regulatory limitations prior to usc.

2. Referenced Documents

2.1 ASTM Slandaid:

D 3835 Test Method for Mcasuring the Rhcological Propcrtics of Thermoplastics with a Capillary Rheometcr ${ }^{3}$ 2.2 ANSI/ADA Standard:

No. 15 Specification for Acrylic Resin Tecth ${ }^{4}$

3. Definition

3.1 intil-one package or vial of premeasured powder component and one package or vial of premcasured liquid component.

4. Physical Requirements

4.1 Liquid:

4.1.1 Appearance-The liquid shall be fire of extrancous particulate matter or obvious visual contaminants in its containcr.

4.1.2 Stability-The liquid shall not increase in original viscosity to such an extent that its flow time will be increased

'This specifieation is under the jurisdiction of ASTM Committec F.4 on Medical and Surgical Matcrials and Devices and is the direct responsibility of Subrommittec F04.02 on Resourccis.

Curren edition approved July 25, 1986. Published Seplember 1986. Originally pullished as F $451-76$. Last previous edition $F=451-76^{4}$.

The boldlace numbers in parentheses relier to the list of references at the end

of his standard.

Anmual Book of ASTMS Standards, vol 0S.03. Available from American National Standards Institutc, 1430 Broadway, New
York, NY 10017 . by more than $10 \%$ when heated 48 h at $60 \pm 2 \%$ in the dark, in a closed containcr (7.3)

4.1.3 Sterility - The liquid, as poured from its container, shall pass the tests described in "Sterility Tests-Liquid and Ointments" (7.4) (2).

4.2 Powder:

4.2.1 Appearance - The powder shall be pourable and frec of cxtrancous matcrials, such as dirt or lint (7.2.2).

4.2.2 Sterility - The powder, as poured from its package, (2).

4.3 Powder-Liquid Mixture.

4.3.1 If the mixture is to be used in its predough stage, the matcrial shall conform to the properties given in Table 1 .

4.3.2 If the mixture is to be used in its dough stage, the material shall conform to the propertics given in Table 1 .

4.3.3 If the mixture can be used in either its predough or dough stages, scparatc units must be tested for compliance with 4.3.1 and 4.3.2.

4.4 Cured Polymer-The matcrial after setting shall conform to the properties given in Table 2 .

5. Weights and Permissible Variations

5.1 Weight and volume measurements shall be madc on the respective powder and liquid components of five units (sce 3.1). Thesc units may be subsequently utilized in any of the nonsterile tests of this specification.

5.2 The weights, or volume of the powder and liquid components, or both, shall not deviate by more than $5 \%$ from those stated on the package (9.2.2), of each of five units.

5.3 Where a radiopaque material is supplied for addition to the powder at the discretion of the surgeon, the weight or volume perecnt of the radiopaque material shall not deviate by more than $15 \%$ from the value stated on the package (9.2.3).

6. Sampling

6.1 Units of powder and liquid shall be procured to provide sufficient matcrial for all the tests of this specificution. The units shall be obtained from regular retail distribution channels. Provided no repcat tests are required, this will amount to between seven and ten units.

6.2 It will only be necessary to maintain stcrility in tests described in 7.4. All other tests described in this specification need not be conducted under sterile conditions.

7. Test Methods and Sample Sire

7.1 Maintain all cquipment, mixing surfaces, and matcrials at $23 \pm 2^{\circ} \mathrm{C}$ at least $2 \mathrm{~h}$ prior to testing and conduct all tests at $23 \pm 2^{\circ} \mathrm{C}$ and $50 \pm 10 \%$ relative humidity unless otherwise specificd. 
7.2 Inspection-Use visual inspection in detcrmining compliance to the requirements outlined in $+1,1,1,1,2,1,3,1 \mathrm{~mm}$ S.2.

7.2.1 The liquid component of two separate units shall comply with the requirements of 4.1 .1 and 8.1.

7.2.2 The powder component of two separatc units shall comply with the requirements of 4.2 .1 and 8.1 .

7.3 Liquid Component Viscosity-Record the viscosity change of two separate units (4.1.2) before and after the heating cxposure by timing the flow of the liquid level between the 0 and $5 \mathrm{~mL}$ marks of a $10 \mathrm{~mL}$ measuring pipet. Calculate the percent change as follows:

$$
\% \text { Change }=\frac{t_{c}-t_{b}}{t_{b}} \times 100
$$

where:

$t_{b}=$ flow time before heating, and

$t_{a}=$ flow time after heating exposure (4.1.2) of $60 \pm 2^{\circ} \mathrm{C}$ for $4 \mathrm{~S} h$ in the dark in a closed container.

7.3.1 An alternative method for viscosity may be used if it can be demonstrated to yield similar results. Both shall comply to the less than $10 \%$ change specified (4.1.2).

7.4 The components of the two units shall be tested for sterility in accordance with the test methods described in U.S. Pharmacopocia, "Stcrility Tcsts" (2).

7.5 Doughing Time:

7.5.1 Enviromment-All cquipment, mixing surfaces, and matcrial (unit size) shall be maintained at $23 \pm 1^{\circ} \mathrm{C}$ at lcast 2 $\mathrm{h}$ prior to testing and tests shall be conducted at $23 \pm 1^{\circ} \mathrm{C}$. The relative humidity shall be $50 \pm 10 \%$.

7.5.2 Mix all the powder and liquid of a single unit together as directed by the manufacturer's instructions (8.5.2). Start a stop watch at the onset of combining the liquid to the powder and read all subsequent times from this stop watch. Approximately 1.5 min after the onset of mixing, gently probe the mixture with a non-powdered surgically gloved (latex) finger. Take visual notice as to the formation of fibers between the surface of the mix and the finger as it leaves the surface. Repeat this process from that time on at 15 -s intervals until the gloved finger separates cleanly. Denote the time at which this is first obscrved as the doughing time. Mix the mixture between determinations to cxpose fresh matcrial for each probing.

7.5.3 Detcrmine the doughing time from two scparate units.

TABLE 1 Requirements for Powder Liquid Mixture

\begin{tabular}{|c|c|c|}
\hline Property & $\begin{array}{l}\text { Extrusion, } \\
\text { Viscosity Tests }\end{array}$ & $\begin{array}{c}\text { Dough Usage. } \\
\text { Intrusion } \\
\text { Tosts } \\
\end{array}$ \\
\hline $\begin{array}{l}\text { Max Dough Time, min. } \\
\text { Setting Timo Range. min. } \\
\text { Temperature, max., } \\
\text { Intrusion, min., mm }\end{array}$ & $\begin{array}{l}5.0 \\
51015 \\
90\end{array}$ & $\begin{array}{l}5.0 \\
5 \text { to } 15 \\
90 \\
2.0\end{array}$ \\
\hline
\end{tabular}

TABLE 2 Requirements for Cured Polymer After Setting

\begin{tabular}{cc}
\hline Property & Requirement \\
\hline Compressive Strength, min., MPa & 70 \\
Indentation, max, mmn & 0.14 \\
Recovery, min., $\%$ & 60 \\
\hline
\end{tabular}

7.5.4 The two values found shall agrec within $30 \mathrm{~s}$ of cact

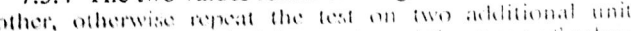
lieport the average of all tour tests and the nunge of watues 7.5.5 Report the doughing time to the nearest $15 \mathrm{~s}$ as the averac of all detcronations Maximum and minimum va. ues of doughing times measured shall not differ by more tha:? $\pm 1 \frac{1}{2} \mathrm{~min}$ from the average.

7.6 Maximum Temperature-Within 1 min after doughing timc, gcntly pack approximatcly $25 \mathrm{~g}$ of the dough described in 7.5 into the mold described in Fig. 1. This mold shall be made of polytetrafluorocthylene (PTFE) and be cquipped with a No. 24 gage wire thermocouple, or similar device, positioned with its junction in the center of the mold at a height of $3.0 \mathrm{~mm}$ in the internal cavity. Immediatcly scat the plunger with a C-clamp or suitable press to produce the $6.0 \mathrm{~mm}$ specimen height. Upon producing plunger seating, remove the cxcess material and the C-clamp or press for the remove the the procedure. Continuously record the perature with respect to time from the onset of mixing the perature with respect to time from the onset, Fig. 2. Report liquid and the powder until cooling is observed, Fig. 2 . Repor
the maximum temperature recorded to the nearest $1^{\circ} \mathrm{C}$. This should not execed the value given in Täble 1.

7.6.1 The me average of two separate detcrminations reported to the nearest $1^{\circ} \mathrm{C}$.

two separate deterninations reported to the difference between the maximum temperature for the two detcrminations is greater than $5.0^{\circ} \mathrm{C}$, repeat the test on two additional units and report the average of all four runs to the ncarcst $1^{\circ} \mathrm{C}$. Individual maximum and minimum runs to the ncarcst 1 . Indiverature shall not differ by more than $\pm 4^{\circ} \mathrm{C}$ of the average valuc of all determinations.

7.7 Selting Time-From the continuous time versus tem. perature recording of 7.6 , the setting time $\left(T_{\text {sct }}\right)$ is the time when the temperature of the polymerizing mass is as follows

$$
T_{\mathrm{amb}}+\left(\Gamma_{\max }-T_{\mathrm{amb}}\right) / 2
$$

where:

$T_{\text {max }}=$ maximum temperaturc, ${ }^{\circ} \mathrm{C}$, and

$T_{\text {max }}=$ ambicnt tempcraturc of $23 \pm 1^{\circ} \mathrm{C}$

7.7.1 Report the sctling time to the nearest $5 \mathrm{~s}$.

7.7.2 Makc two separatc detcrminations of the setting time.

7.7.3 The two values should agrec within $1 \mathrm{~min}$ of each other, otherwise repeat the test on two additional units and report the average of all runs.

7.7.4 Report the setting time to the nearest $15 \mathrm{~s}$ as the average of all detcrminations.

7.8 Flow Properties and Viscosity Determination-The manufacturer must specify whether the ecment may be used in its pre-dough or dough state, or both. The determination in the following tests the cement fortice in the should comply we the extrusion, viscosity test (7.8.1) and pre-dough stage, use the extrusion, viscosity the dough stage, Table 1. If the mixture is to be utized in the dough stage, use the intrusion test (7.8.2) and Table . If the mixture is to (7.8.1) and intrusion (7.8.2) tests must be performed.

7.8.1 Extrusion, Viscosity:

7.8.1.1 Apparatus:

7.8.1.1(a) Rheometer-Any capillary rheometer is satisfactory in which acrylic bone cement can be foreed from a reservoir through a capillary dic and in which temperature, 


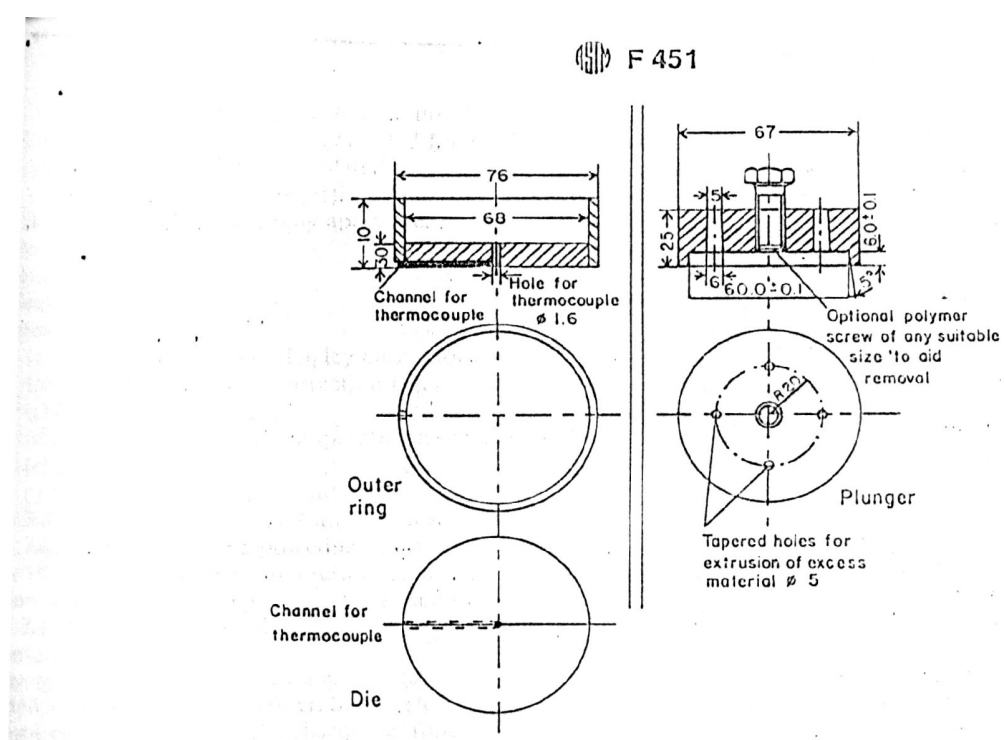

Nore-Dimensions in millimetres and \pm 0.2 unless otherwise specilicd. Material for all components: Polytetrafluoroethylonc, poly(ethylenetercphthalatc). polyoxymethlene, or high density polyethylene.

FIG. 1 Exothermic Heat Miold

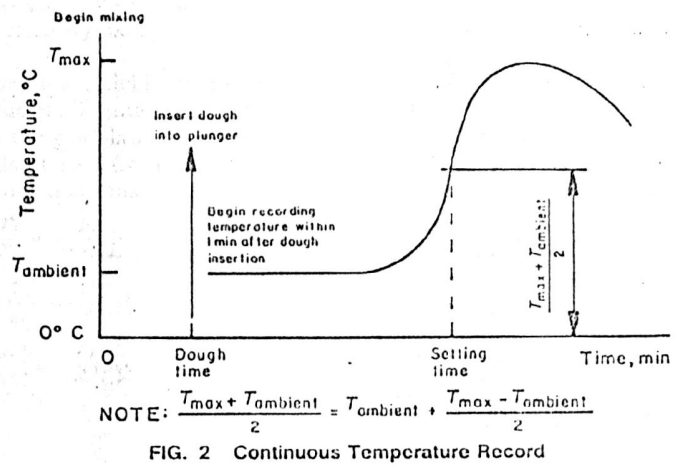

applicd force, output rate, and barrel and die dimensions can be controlled and measured accuratcly. Equipment that provides a constant shear rate has been shown to be cqually uscful. The capillary dic of the rheometer shall have a smooth straight bore that is held within $\pm 0.0076 \mathrm{~mm}$ $( \pm 0.0003 \mathrm{in}$. $)$ in diameter and shall be held to within \pm 0.025 $\mathrm{mm}( \pm 0.001$ in.) in length. The borc and its finish arc eritical. It shall have no visible drill or other tool marks and no detcctable eccentricity.

7.S.1.1(b) Due to the extreme sensitivity of flow data to the capillary dimensions, it is important that the capillary dimensions are measured with precision and reported. The length to diameter ratio shall normally be between 20 and
40. Larger ratios and ratios less than that suggested require applying large corrections to the data $(3,4)$. In addition, the ratio of the reservoir diameter to capillary diameter should be between 3 and 15. Sce Test Method D 3835 for further details of capillary rhcometers.

7.8.1.2 Calibration-Perform the test with a cortificd standard viscosity fluid approximating that expected for bonc cement $\left(50 \mathrm{~N} \cdot \mathrm{s} / \mathrm{m}^{2}\right.$ to $\left.500 \mathrm{~N} \cdot \mathrm{s} / \mathrm{m}^{2}\right)$. Determine the viscosity of the standard fluid and the percent error from its specified valuc. Report this error along with the viscosity of the icsted cements.

7.8.1.3 Corrections-Since bonc cement is a nonNewtonian fluid, the data may be reported as corrected data. 
or cxample, true shear rates, corrected for non-Newtonian low behavior and true shear stress corrected for end effects ir kinetic energy losses, may be calculated. In such cases, the xact details of the mode of correction must be reported. iome correction factors which may apply are:

(a) Piston friction,

(b) Plunger back flow,

(c) Cement compressibility,

(d) Barrcl back pressurc,

(c) Capillary entrance effects (Baglcy correction) (5),

(f) Rabinowitsch shear ratc correction (6).

7.S.1.4 Procedure:

7.8.1.4.1 Sclect conditions of temperature and shear stress ir shear rate in accordance with expected usage so that the low rate will fall within desired limits.

7.8.1.4.2 Inspect the rheometer and clean it if necessary. insure that previous cleaning procedures and usage have not hanged the dimensions or caused scratches or defects in the apillary or apparatus. Makc the neccssary measurements on he apparatus for future calculations. Prepare the apparatus or running the test.

7.S.1.4.3 Mix one or more complete unit(s) of powder and quid in the recommended manner. Start a stop watch at the nset of mixing and read all subscquent times from this ratch. After complete mixing, transfer the cement to the acrmally cquilibrated reservoir, and eject any entrapped air $r$ excess bone ccment.

7.S.1.4.4 Start the apparatus at a time not greater than $21 / 2$ nin from the start of mixing and continuc operating until he estimated dough time or the viscosity exeecds $500 \mathrm{~N} \cdot \mathrm{s} /$ $1 n^{2}$.

7.S.1.4.5 Disasscmble the apparatus quickly before the cment sets and clcan the apparatus of all remaining cement. 7.8.1.5 Calculations-Perform the calculation for viscosty of the cement at time intervals of $15 \mathrm{~s}$ from the start to inish of test run. Usc the following equations:

$$
\begin{gathered}
\text { Shear Stress, } \mathrm{Pa}=\frac{P r}{2 L}=\frac{F r}{2 \pi R^{2} L} \\
\text { Shear Rate, } \mathrm{s}^{-1}=\frac{4 Q}{\pi r^{3}}=\frac{4 V}{\pi r^{3} t} \\
\text { Viscosity, Pa.s }=\frac{P \pi r^{4}}{8 L Q}=\frac{F r^{4} l}{8 R^{2} L V}
\end{gathered}
$$

vhere:

D = pressure by ram, $\mathrm{Pa}$,

$F=$ force on ram, $N$

= radius of capillary, $\mathrm{m}$

$\mathrm{R}=$ radius of barrcl, $\mathrm{m}$,

$L=$ length of capillary, $m$,

$\rho=$ flow ratc, $\mathrm{m}^{3} / \mathrm{s}$,

$V=$ volume cxtruded, $\mathrm{m}^{3}$, and

$=$ extrusion time, $s$.

7.S.1.5.1 These equations yicld true shear rate and truc riscosity for Newtonian huids only; for non-Newtonian huids, such as bone cement, the apparent shear rate and viscosity are obtaincd.

7.8.1.6 Report-The report of the flow propertics of the cement shall include:

7.S.1.6.1 Description of the rheometcr used.

7.\$.1.6.2 Temperature at which the data were obtained. .
7.8.1.6.3 The capillary diameler and length to diameter ratio of the capillary.

7.8.1.6.4 The shear ratc at which the test was performed.

7.8.1.6.5 Viscosity versus obscrvation time for threc runs.

7.8.1.6.6 Statement as to whether any correction factors

(7.8.1.3) were applicd.

7.8.2 Inirusion:

7.8.2.1 The mold necessary for this test shall be made of PTFE and is shown in Fig. 3. Mix one completc unit of powder and liquid in the recommended manner (4.3.1). When doughing is achicved, gently pack the entire mixture flat into the mold and insert the plunger. One minute after the doughing time, place a load of $49 \mathrm{~N}(11 \mathrm{lb})$ on top of the plunger for one minute. Then remove the weight and allow the mixture to set.

7.8.2.2 Following the set, remove the specimen and mea. sure the average height of the intrusion into all four of the $1.0-\mathrm{mm}$ diameter holes of the dic to the nearest $0.5 \mathrm{~mm}$.

7.8.2.3 Run this test once. If the requirement is not met, it must be met so in a repeat test.

7.9 Compressive Strength-The test specimens shall be cylinders $12 \mathrm{~mm}$ high and $6 \mathrm{~mm}$ in diameter. The ends of the specimens shall be flat and smooth and shall be paralle to each other and at right angles to the long axis of the cylinder. An apparatus found convenient for forming these test cylinders is shown in Fig. 4. An apparatus containing

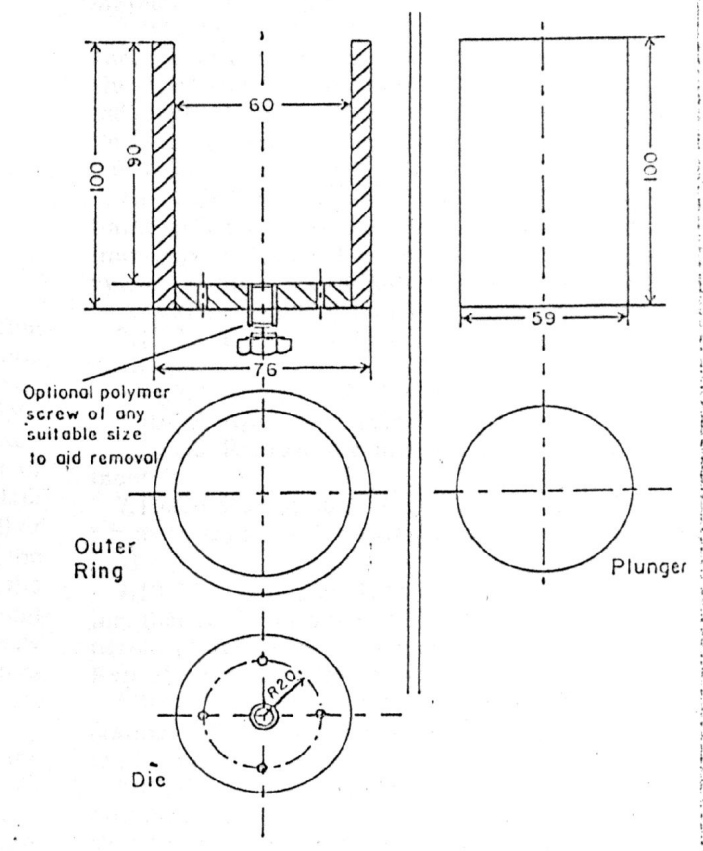

NOTE-Dimensions in millimctres; 4 holes in bottom to be $1.00 \pm 0.05$. Toleranc: on all other dimensions \pm 0.2 . Material for all components: Polytetrafluoroethylerie poly(ethyienetercphthalatc), polyoxymethylene, or high density polyethylene. FIG. 3 Intrusion Mold 


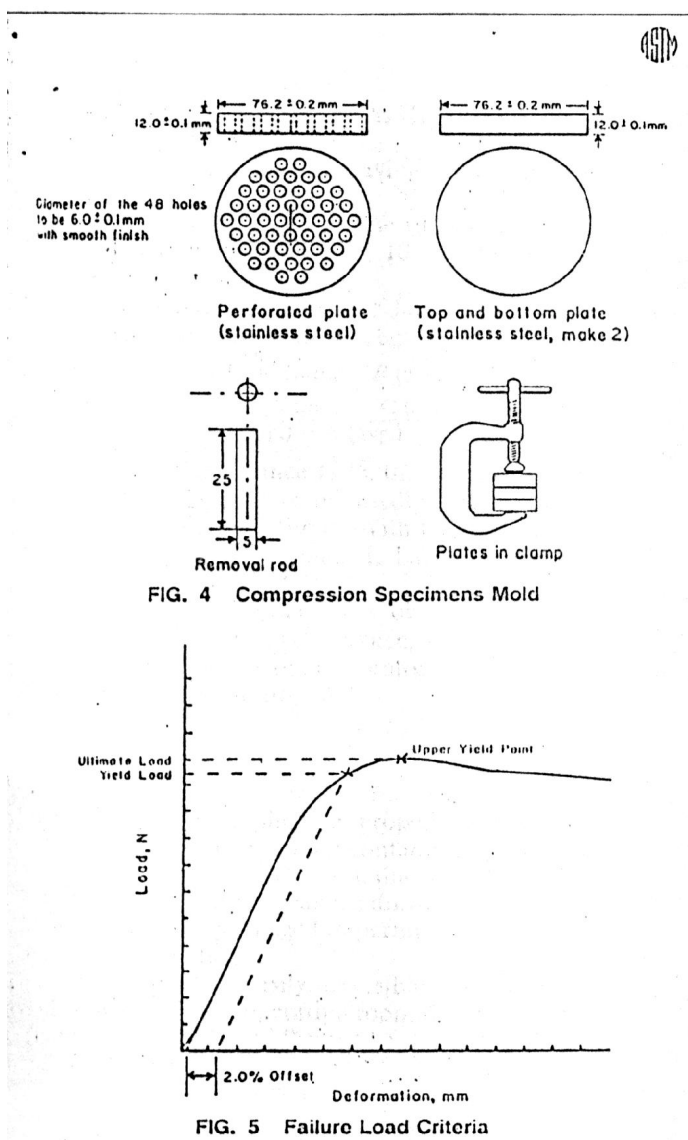

more specimen holes may be used as long as adequate spacing between holes is maintained. A mold release agent or silicone spray may be sparingly applicd to facilitate specimen removal. 7.9.1 Place the specimen mold on a flat glass or smoolh metal plate and slightly overfill using onc unit of mixed cement of standard proportions at the commencement of dough time. Press a second flat glass or smooth metal plate on top of the mold. Hold the mold and plates firmly together with a small C-clamp. Then, 1 h later, surface the ends of the cylinder plane at right angles to the axis. The ends of the specimens may be ground hat to the axis by use of a small amount of 240-mesh silicon carbide powder and watcr. Draw the molds containing the spccimens back and forth across the plate coated with the abrasive and water. After surfacing, remove the specimens from the mold.

7.9.2 The time lapse between the start of mixing and the measurement of the compressive strength testing shall be 24 $\pm 2 \mathrm{~h}$. Storage of the specimens before testing shall be at $23 \pm$ $2^{\circ} \mathrm{C}$ and $50 \pm 10 \%$ relative humidity. Run specimens on any universal testing machine cquipped to record load versus deformation. Employ a deformation cross head specd of 20 or $25.4 \mathrm{~mm} / \mathrm{min}$. Test the specimens without the use of any

\section{$F 4.51$}

type of pad between the specimen and the platens of the machinc. The failure load shall be the load at the $2.0 \%$ oftset ( $2.0 \%$ proof stress), upper yicld point, or at fracturc, whichcver occurs first (Fig. 5).

7.9.2.1 Calculate the compressive strength as the failure load divided by the calculated cross-sectional arca.

7.9.2.2 Report the compressive strength as the average of a minimum of five specimens to the nearest $1 \mathrm{MPa}$ (150 psi). 7.9.3 Repeat the compressive strength test after immersing a minimum of five test specimens in water at $37^{\circ} \mathrm{C}$ for scren days $\pm 2 \mathrm{~h}$.

7.9.3.1 After removing the specimens from the mold (7.9.1) place the specimens in water at $37^{\circ} \mathrm{C}$. Maintain the samples in the water bath for seven days $\pm 2 \mathrm{~h}$.

7.9.3.2 Test the specimens in accordance with 7.9.2.

7.9.3.3 Report the compressive strength as the average of a minimum of five specimens to the nearest $1 \mathrm{MPa}$ ( $145 \mathrm{psi}$ ). If the strength is less than $90 \%$ of the value reported in 7.9.2.2, repeat the test. The minimum acceptable strength is $90 \%$ of the value reported in 7.9.2.2 or the value stated in Table 2, whichever is greater.

7.10 Indentation and Recovery (sec ANSI/ADA Specification No. 15)-Determinc the indentation resistance at $24 \pm$ 2 ls iftcr start of mixing with a "Rockwcll" type supcrficial hardness tester. Usc the disk made in 7.6, avoiding the region of the thermocouple placement. Remove any flash or other irregularity which would prevent the disk from seating firmly on the hardness tester.

7.10.1 Since some of thesc "Rockwcll" type superficial hardness testers are designed for multiple applications, excrcise care to ensure that the instrument used is rigged to apply the loads prescribed below and that the dial gage referred to for measurement of penetration is one having an accuracy of $0.001 \mathrm{~mm}$ (0.00004 in.).

7.10.2 Before conducting the test, determine the inherent frame spring factor of the instrument, that is, the amount the frame opens during the application of the major load. The average of three detcrminations shall be recorded as $A$.

7.10.2.1 Remove the anvil and indenter components.

7.10.2.2 Place a Rockwcll "C" calibration block on the anvil scat.

7.10.2.3 Apply the $29.4 \mathrm{~N}$ minor load.

7.10.2.4 Apply the $290.4 \mathrm{~N}$ major load.

7.10.2.5 Remove the major load and re-zero the instrumont.

7.10.2.6 Repeat steps 7.10.2.4 and 7.10.2.5 until the instrument consistently reads zcro upon removal of the major load.

7.10.2.7 Then re-apply the major load. The resulting reading, that is, the number of dial divisions through which the needle passes, reflects the inherent frame spring factor, $A$. Repeat three times for an average $A$.

7.10.3 Replace the spot anvil and 12.7- $\mathrm{mm}$ ball indenter components and use a Rockwcll 30 "Y" block to calibratc the instrument.

7.10.4 Test the specimens in the following manner. The average of three detcrminations (cach at a separatc location) shall be made for total indentation and recovery.

7.10.4.1 Apply the minor load.

7.10.4.2 Apply the major load (which includes the minor - load) for 10 min.

01

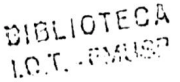


,

7.10.4.3 Record the value registered as Reading $B$ (indenttion plus spring factor).

7.10.4.4 Remove the major load lcaving the minor load sting on the specimen.

7.10.4.5 Then, $10 \mathrm{~min}$ later, record the value of the numor of dial divisions subtracted from 100 as Reading $C$ 2ecovery Reading).

7.10.4.6 The total indentation under load and recovery rall be detcrmincd using the following equations:

Total. Indentation Under Load $(\mathrm{mm})=B$ (avg.) $-A$ (avg.)

$$
\text { Recovcry, } \%_{0}=\frac{B \text { (avg.) }-A(\text { avg. })-C \text { (avg.) }}{B \text { (avg.) }-A \text { (avg.) }} \times 100
$$

7.11 Precision and Bias-Since 1976, the original Specifiition F 451 methodologies have reportedly been routincly ilized by the various manufacturers. With the exception of c viscosity method of 7.8.1, which is based on another ccpted ASTM document (Test Method D 3835), each test ethodology in Section 7 contains its own statement of porting acceptable levels of performance, reproducibility, id precision. Therefore, no interlaboratory studies have en performed by the Committee F-4. i.

\section{Packaging}

S.1 Materials shall be supplied in properly sealcd conners made of materials that shall not contaninate or permit ntamination of the contents. The containcrs shall be packed so as to prevent damage or lcakage during shipping and rage. Matcrials must be packaged to permit sterile transfer contents to the sterile field.

8.2 The contents shall be easily accessible, easy to open, id convenient to mix in the operating room. Entirc packagc ntents (both powder and liquid) must be mixed to achicve commended proportions.

\section{Labcling}

9.1 Labeling on these cements must bc in conformance th the Federal Food, Drug, and Cosmetic Act, Code of deral Regulations, and other pertincnt laws and regulains.

9.2 The following minimal information must appear on c container label.

\section{$F 451$}

9.2.1 It shall be clcarly stated or color coded, or both, if the mixture is intended for usage in the pre-dough, dough, of dual usage statc.

9.2.2 The wcight or volume, or both, of the liquid and powder components must be stated.

9.2.3 Constituents of the powder and liquid shall be clcarly stated in terms of weight or volume percent. This shall include the gencric names of polymers, copolymers, chemical initiators, stabilizers, cross-linking agents, and any other ingredicnts, such as radiopacify agents, gels, fillers, or antibiotics.

9.2.4 A statement that the contents are sterile and that the sterility shall be guaranteed only if the containcrs are undam. aged.

9.2.5 The following warning shall appear on the label: (a) Flammable liquid; (b) Storc below $25^{\circ} \mathrm{C}$, and (c) Protect from light.

9.2.6 A statement to the effect that federal law restricts this device for sale by or on the order of a physician should bc displayed.

9.2.7 The manufacturer and distributor shall be identificd.

9.2.8 Each individual component of the package unit must be clearly identified as to batch or lot number.

9.3 The following information shall appcar on the product inscrt labeling accompanying cach packagc.

9.3.1 Adequatc and accurate instruction shall be given for handling the components and preparing the cement. Instructions shall include a dircetive to mix all of the powder with all the liquid of a single unit. Procedures required to mix the matcrials, along with recommended mixing utcasils, shall be given.

9.3.2 Proper technique for administration and recom. mended procedures for using the ccment, including any special precautions, shall be indicated.

9.3.3 Toxic, hazardous, or irritating characteristics associatcd with the handling and usc of the components and cement shall be indicated.

9.3.4 A statement shall be included that states that high temperatures of either the ambient surroundings or matcrial will causc shorter doughing, working, and setting times, while low temperatures of either the ambient surroundinas or material will incrcasc doughing, working, and sctting times.

9.3.5 The ranges of doughing and setting times as mez. surce at $23 \pm 1^{\circ} \mathrm{C}(7.5$ and 7.7$)$ shall be clcarly stated.

\section{APPENDIX}

(Nonmandatory Information)

\section{Xl. RATIONALE}

$X 1.1$ Acrylic bone cement is a powder-liquid system that currently sold in the United Staics for the fixation of ernal orthopedic appliances. Bccause it plays a kcy rolc in , hly synchronized surgical procedures, such as total joint lacement, its sctting characteristics must be known and nsistent each time. The matcrial must also have adequatc ysical propertics for placement and function. To these cnds, the standard is addressed.

X1.2 While many of the tests are obvious forms of good manufacturing practice, others may be slightly morc subtle and requirc some claboration.

X1.2.1 The stability test measures the viscosity after storing the liquid at $60^{\circ} \mathrm{C}$. This is an accelerated aging test 10 cnsure that the monomeric component of the bone cement 
does not readily polymerize prematurely while stored before

$-\lambda 1.2 .2$ The dough and set times check that the material will be ready for placement at the proper time in the surgical procedure and that it will set neither prematurcly nor in a delayed fashion.

Xi.2.3 The maximum temperature test makes sure that the mass will not release excessive heat during setting. This could be damaging to the patient's tissuc if not properly controlled.

X1.2.4 The dough and setting times and maximum temperature test also cvaluatc other important parameters. These tests will only respond consistently in the required time and lemperature ranges if the powder particle size distribution, the liquid to powder ratio, the complex chemical compositions of both the powder and liquid, and the catalyst amount and distribution have becn properly formulated and meted out and strict quality control during all stages of manufacluring is carcfully monitored.

X1.2.5 The viscosity tests of the predough matcrial and intrusion cvaluations demonstratc that the matcrial will flow into the bony interstices and around prostheses to produce adequatc mechanical interlocking upon setting of the material.

X1.2.6 The compressive strength test measures if the set material will be strong cnough for clinical applications.

X1.2.7 The indentation and recovery measures the resist-

\section{F 451}

ance of the surface to an appliced load and recovery after release of this load. Multiple measurcments of indentation and recovery on various surface locations will verify that the liquid was properly distributed during mixing and pockets of unreacted liquid or low molecular weight polymer are not likcly to be present.

X1.3 Further topics presently under considcration for cventual addition to the specification are as follows:

X1.3.1 Other mechanical tests, such as tension, flexion, and fracture toughness, which may be more sensitive to internal porosity and surface defects than the current compression test.

X1.3.2 Statement of biocompatibility of the ecment.

X1.3.3 Shortening the time spans of the indentation tests.

X1.3.4 Requiring doughing and setting time data to bc furnished with product information.

X1.3.5 Investigating changes in physical properties when lcachable additives, such as antibiotics, are purposefully added.

X1.3.6 To define the optimum conditions of the viscosity test methodology so that the performance standards can bc cstablished.

X1.4 It should be noted that this document contains both test methodology and performance standards. Currently, in the case of viscosity measurement, only the test methodology has been described. This methodology will serve as a basis for future performance standards.

\section{RLFERLNCES}

(1) Willians, D. F., and Roaf, R., Implants in Surgery, W. B. Saunders Co.. Lid., London, 1973, pp 216-218.

(2) U.S. Pharmacupocia Revision 20, Mack publishing Co., Buston, PA, 1980, p sSO.

(3) Tordella. J. P., cd., Transactions of the Society of R/cology, Vol I, 1957, p 203.
19.
(4) Philipporf, W., and Gaskins, F. H., cds., Fransactions of the Srecicty of Rhcolog, $\mathrm{Vol} 2,1958, \mathrm{p} 263$

(5) Bagley, E. B., and Birk, A. M., cds., Journal of Applicd Physics, Vol 31,1960, p 556

(6) Rabinowitsch, R., Zeitschrift Fiuer Physikalische Chemic, Vol A $145,1929, \mathrm{p}$ 1.

The American Society lor Testing and Matorials lakes no position respecting the validity of any patent rights assortcd in connoction with any item montioned in this standard. Usors of this slandard are oxprossly advisod that dotermination of the validity of any such patent rights, and the risk of infringement of such rights, aro ontirely their own responsibility.

This standard is subjoct to revision at any timo by the rosponsiblo tochnical committeo and must be roviowed every live years and if not revised, either reapproved or withdrawn. Your commonts are invited oither for rovision of this standard or for additional standards echnical commiltee, which you may attond. II you loel that your commonts have not reccived a loir hoaring you should mako your viouvs known to the ASTM Committoo on Standards, 1916 Race St., Philadolphia, PA 19103. 
ANEXO B

ISO 5833 


\section{INTERNATIONAL STANDARD}

First edition 1992-11-15

Implants for surgery - Acrylic resin cements Implants chirurgicaux - Ciments à base de résine acrylique

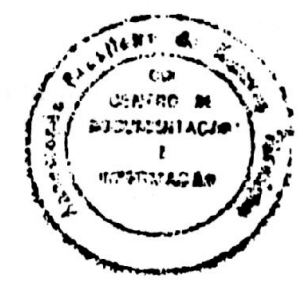




\section{Contents}

Page

1 Scope

2 Definition

3 Liquid component

4 Powder component

5 Liquid-powder mixture intended for syringe usage

6 Liquid-powder mixture intended for use in dough state

7 Sel and cured cement

8 Packaging

$9 \quad$ Labelling

\section{Annexes}

A Method for determination of slability of liquid component

B Method for determination of doughing time of liquid-powder mixture of cement intended for dough usage

C Method for delermination of maximum temperature and setting time of liquid-power mixture

D Method for delermination ol intrision of liquid-powder mixture of cement intended for dough usam

E Method for determination of $\mathrm{co}$

strength of cement

F Method for determination of bend: of cement

lus and bending strenglh

- ISO 1992

All rights reserved. No part of this publication may be reproduced or utilized in any form or by any means, electronic or mechanical, Including pholocopying and microfilm, without permlssion in writing from the publisher.

International Organization for Standardization

Case Fostale $56 \bullet \mathrm{CH}-1211$ Genève $20 \bullet$ Switzerland

Printed In Switzerland 
i. $3633: 1932 !$

\section{Foreword}

ISO (the International Organization for Standardization) is a worldwide federation of national standards bodies (ISO member bodies). The work of preparing International Standards is normally carried out through ISO technical committees. Each member body interested in a subject for which a technical committee has been eslablished has the right to be represented on that committee. International organizations, governmental and non-governmental, in liaison with ISO, also take part in the work. ISO collaborates closely with the International Electrotechnical Commission (IEC) on all matters of electrolechnical standardization.

Draft International Standards adopled by the technical committees are circulated to the member bodies for voting. Pullication as an International Standard requires approval by at least $i 5 \%$ of the member bodies castịng a vote.

International Standard ISO 5833 was prepared by Technical Committee ISO/TC 150, Implants for surgery, Sub-Commiltee SC 1. Materials.

This first edition of ISO 5833 cancels and replaces the first edition of ISO 5833-1, published in 1979 and the planned IS $r$;833-2, of which it constitutes a minor revision.

Annexes A, B, C, D, E and $F$ form an integral part of this International Standard. 


\section{Implants for surgery - Acrylic resin cements}

\section{Scope}

This International Standard applies to radio-opaque and non-radio-opaque cements and specifins physical, mechanical, packaging and labellir in inulements for sell-curing resin cement used on poly(methacrylic acid esters), of two type intended respectively for use with a syringe or in lie dough stale for the fixation of intcrila! o'tiopaedic prostheses and supplied as units corlsining premeasured amounts of sterile powder an: of sterile liquid in forms suitable for mixing at the :ime of implantation.

This International Slandard does not cover the hazards associated with the use of the cement in respect of either the patient or the user ol the cement.

All requirements apply to, and all tests are to be perlormed on, the sterile product.

\section{Definition}

For the purposes of this International Standard, the following definition applies.

2.1 unlt of cement: One package or vial of sterile fr. measured jowder component and one package or vial of sterile pre-measured liquid component.

\section{Liquid component}

\subsection{Appearance}

When inspected by normal or corrected vision, the liquid shall be free from particles and other contaminants

\subsection{Stability}

When tested as described in annex $A$, the flow time of either sample of liquid shall not increase by more than $10 \%$.

\subsection{Accuracy of contents}

When measured to an accuracy of $\pm 0.1 \mathrm{ml}$, the volume of the liquid component of each and every one of five units shall be willin $5 \%$ of that stated on the package [see 9.1 a)]

\section{Powder component}

\subsection{Appearance}

When inspected by normal or corrected vision, the powder shall be free from agglomerates and extraneous material

\subsection{Accuracy of contents}

When weighed to an accuracy of $\pm 0,1 \mathrm{~g}$, the mass of the powder component of each and every one of five units shall be within $5 \%$ of that stated on the package [ ree 9.1 a)].

NOTE 1 The components used for the detarminutions specified in 3.3 ant 4.2 may be used subserfuently for other tests described in this International Standard.

\section{Liquid-powder mixture intended for syringe usage}

When delermined by the methods given in tables 1 and 2 , the selling propertics, and the properties of the set cement, shall comply with the values given in tables 1 and 2 . 
6 Liquid-powder mixture intended for use In dough state

\subsection{Setting properties}

When determined by the methods given in tables 1 and 2 , the setting properties, and the properties of the set cement, shall comply with the values given in tables 1 and 2.

\subsection{Intrusion}

When determined as described in annex $D$, the average Intrusion of iat least one sample shall be not less than $2 \mathrm{~mm}$.

\section{Set and cured cement}

Table 2 sets out the requirements and test methods for set and cured cement.

\section{Packaging}

Each component of the cement shall be packaged ànd sterilized in a double-layer sealed container and - then packaged in an outer container which shall contain the accompanying documentation.

The materials of the package should not contamInate or permit contamination of the contents. The packaging should prevent damage to, or leakage of the contents during transit and storage and should be designed so that it is easy to open and facilitates aseptic presentation of the contents:

\section{Labelling}

\subsection{Unlt package}

At least the following information shall appear on the unit package of each cement unit:

a) a description of the contents, including the mass of the powder component and the mass or volume of the liquid component, and the generic names of the constituents;

* b) the relative proportions of the powder and liquid components expressed as a mass or volume percent;

c) the name and address of the manufacturer, and the suppller if different Irom the manufacturer:

a) a statement that the contents are sterile, and a warning against the use of an opened or damaged package;

* e) a warning that the package contains flammable liquid;

n) an instruction to store the package in the dark at below_25. $\mathrm{C}$;

g) the batch or lot numbers of the liquid and the powder component and the expiry date of the material;

* h) the number and date of this International Standard (l.e. ISO 5833:1992).

NOTE 2 Legal requirements for labelling may apply in some countries.

Table 1 - Requirements and test methods for setting properties of liquid-powder mixtures

\begin{tabular}{|c|c|c|c|c|c|c|c|c|}
\hline \multirow{2}{*}{ Mixture } & \multicolumn{3}{|c|}{ Doughing time } & \multicolumn{2}{c|}{ Selting time } & \multicolumn{3}{c|}{ Maximum temperature } \\
\cline { 2 - 8 } & $\begin{array}{c}\text { Average } \\
\min \end{array}$ & $\begin{array}{c}\text { Maximum } \\
\text { deviation from } \\
\text { average } \\
\text { min }\end{array}$ & $\begin{array}{c}\text { Test } \\
\text { method }\end{array}$ & $\begin{array}{c}\text { Average } \\
\min \end{array}$ & $\begin{array}{c}\text { Test } \\
\text { method }\end{array}$ & $\begin{array}{c}\text { Average } \\
{ }^{\circ} \mathrm{C}\end{array}$ & $\begin{array}{c}\text { Maximum } \\
\text { deviation Irom } \\
\text { aver age } \\
{ }^{\circ} \mathrm{C}\end{array}$ & $\begin{array}{c}\text { Test } \\
\text { method }\end{array}$ \\
\hline $\begin{array}{c}\text { Syringe usage } \\
\text { (see clause 5) }\end{array}$ & - & - & - & 6,5 to 15 & Annex C & 90 & \pm 5 & Annex C \\
\hline $\begin{array}{l}\text { Dough state } \\
\text { usage (see 6.1) }\end{array}$ & 5 max. & 1,5 & Annex B & 3 to 15 & Annex C & 90 & \pm 5 & Annex C \\
\hline
\end{tabular}


ISU $5: 3: \operatorname{lnm} n$ (E)

Table 2 - Requirements and test methods for set and cured cement

\begin{tabular}{|c|c|c|c|c|c|}
\hline \multicolumn{2}{|c|}{$\begin{array}{c}\text { Average compressive } \\
\text { strengih }\end{array}$} & \multicolumn{2}{|c|}{ Bending modulus } & \multicolumn{2}{c|}{ Bending strength } \\
\hline $\begin{array}{c}\text { min. } \\
\mathrm{MPa}\end{array}$ & Test method & $\begin{array}{c}\mathrm{min} . \\
\mathrm{MPa}\end{array}$ & Test method & $\begin{array}{c}\text { min. } \\
\mathrm{MPa}\end{array}$ & Test method \\
\hline 70 & Annex $\mathrm{F}$ & $1 \mathrm{B00}$ & Annex $\mathrm{F}$ & 50 & Annex F \\
\hline
\end{tabular}

\subsection{Accompanying documentation}

At least the following information shall appear on the accompanying documentation (see clause 8 ):

a) instructions for handling the components and preparing the cement for use incle "ing detalls of the equipment needed and an instruction to mix the entire contents of the pacl. . The instructions shall emphasize the in tance of minimizing the entrapment of air;

* b) instructions and recommendations for using the cement, including necessat precau': ns;

c) a statement diawing alte to to ardous and irritant propert ass with the handling and use of the components and the cement;

d) a ...tement Itai' 'in ambient or component ten. alures will Jecrease, and low ambient or component fompelatures will Increase, the doughling. working and setting times of the cement:

y e) a graphical representation of elfect of temperature on the length of the phases in cement curing, prepared from experimental data on the paiticular brand of ment.

NOTE 3 Examples of graphs are shown in figures 1 and 2 .

1) whether the cement is intended for use with a syringe or in the dough state.

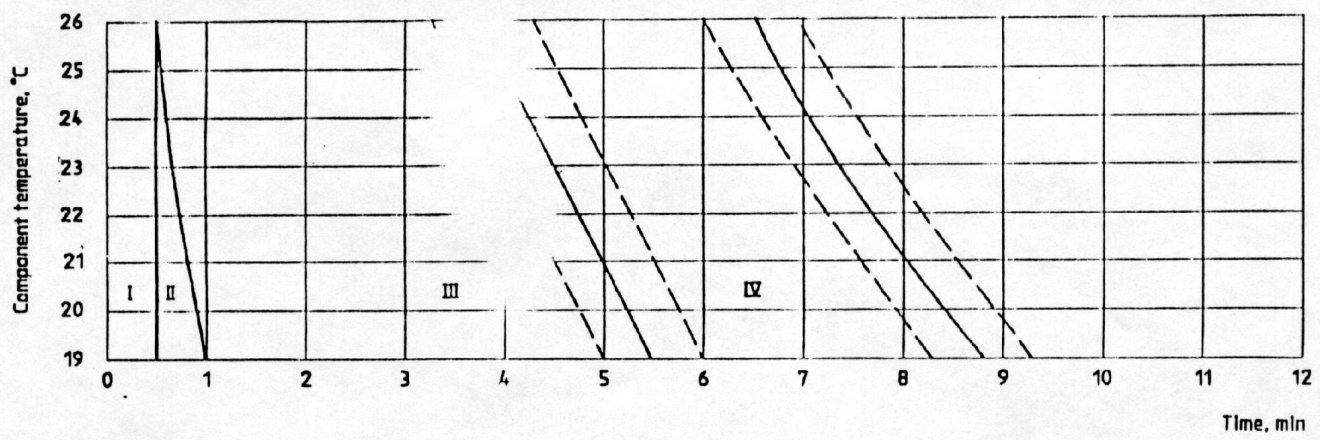

I Mixing time

II Slicky phase Fase Viscisn

III Working lime

IV Hardening time

Hardening,time

A deviation of $\pm 30 \mathrm{~s}$ on working and har ciening times may occur. The optimum working temper alure has been determined to be $23^{\circ} \mathrm{C} \pm x^{\circ} \mathrm{C}$.

Figure 1 - Example of graph showing working data for cement intended for dough usage 
-333:1992(ㄴ)

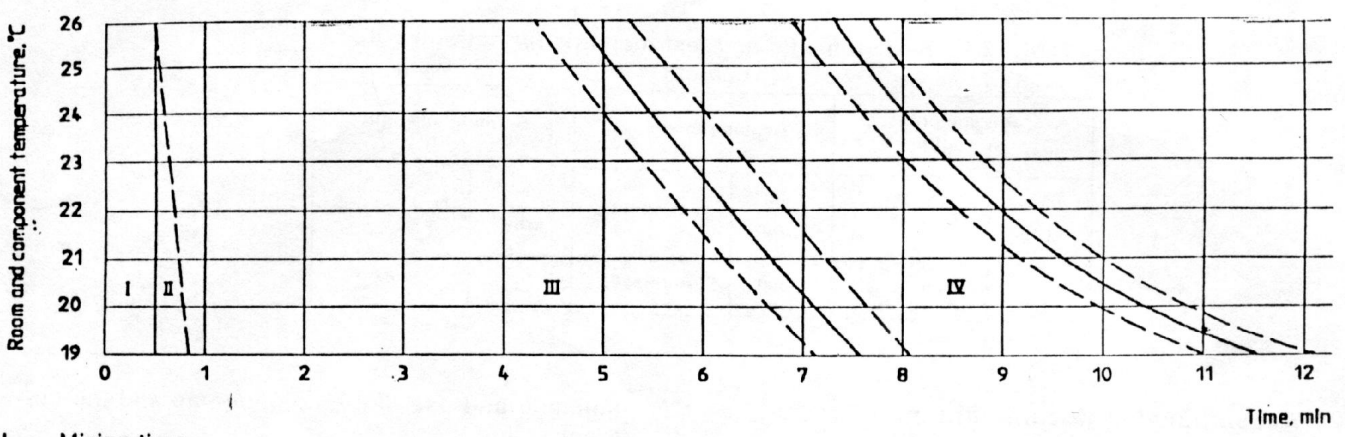

I Mixing time

II Filling of syringe

III Working time

V Hardening time

A deviation of $\pm 30 \mathrm{~s}$ on working and hardening times may occur. The optimum working temperature has been determined to be around $23^{\circ} \mathrm{C}$.

Figure 2 - Example of graph showing working data for cement Intended for syringe usage 


\section{Annex A}

(normative)

\section{A Method for determination of stability of liquid component}

NOTE 4 Other methods of equivalent accuracy and precision may be used, but the method given in this annex is the referee method in cases of dispute.

\section{A.1 Principle}

The flow time (viscosity) of the liquid component is determined before and after accelc'atc 1 ageing by heating, and the Increase in flow tinie after heating is calculated.

\section{A.2 Apparatus}

/A.2.1 Clean glass U-tube viscometer

- A.2.2 Timing device, of accuracy $\pm 0.2 \mathrm{~s}$

A.2.3 Means of heating test specimens.

\section{A.3 Test conditions}

Maintain the viscometer and the lort pecimens at $23^{\circ} \mathrm{C} \pm 1{ }^{\circ} \mathrm{C}$ for at least $16 \mathrm{~h}$ belo; hegining the test. Perform the viscosity measur:ments at $23^{\circ} \mathrm{C} \pm 1{ }^{\circ} \mathrm{C}$

\section{A.4 Procedure}

A.4.1 Fill the viscometer in the usual way with the liquid component.
A.4.2 Record the flow time taken lor the meniscus to fall to the equilibrium level (time $t_{b}$ ). "

- A.4.3 Heat an aliquot of the lirpuid component at $60^{\circ} \mathrm{C} \pm 2{ }^{\circ} \mathrm{C}$ for $48 \mathrm{~h} \pm 2 \mathrm{~h}$ in the dark in a closed container; allow it to cool to $23^{\circ} \mathrm{C} \pm 1{ }^{\circ} \mathrm{C}$ and to remain at this temperature for at least $16 \mathrm{~h}$.

A.4.4 Rernat A.4.1 and A.4.2 and record the flow time (time $t_{\mathrm{a}}$ ).

A.4.5 Repeat A.4.1 to A.4.4 on the liquid component of a second unit of cement.

\section{A.5 Calculations and expression of results}

Calculate the percentag change in flow time for each unit of cement using the expression:

$$
\text { percentage change }=\frac{t_{\mathrm{a}}-t_{\mathrm{b}}}{t_{\mathrm{b}}} \times 100
$$

\section{A.6 Test report}

The test roport shall include at least the following information:

a) the identity (including batch or lot number) of the liquid component:

b) the flow times before and after heating:

c) the percentage change in flow time for each unil of cement. 
iuン uUง3:1992(t)

\section{Annex B}

(normative)

\section{: Method for determination of doughing time of liquid-powder mixture of cement intended for dough usage}

\section{B.1 Principle}

The cement is mixed and the time recorded from the beginning of mixing until the mixture is able to separate cleanly from a gloved finger.

\section{B.2 Apparatus}

B.2.1 Timing device of accuracy $\pm 0,2 \mathrm{~s}$.

B.2.2 Unpowdered latex surgical gloves.

B.2.3 Equipment as recommended by the cement manufacturer, for mixing cement.

\section{B.3 Test conditions}

Maintain the mixing equipment and the contents of the cement units at $23^{\circ} \mathrm{C}+1{ }^{\circ} \mathrm{C}$ and at a relative humidity (R.H.) of not less than $40 \%$ for at leasl $16 \mathrm{~h}$ before beginning the test. Perform the test at $23^{\circ} \mathrm{C} \pm 1{ }^{\circ} \mathrm{C}$ and a R.H. of not less than $40 \%$

\section{B.4 Procedure}

B.4.1 Mix all the components of a single unit of cement following the inanufacturer's instructions Start the timing device when the liquid is first added to the powder.

B.4.2 After approximately $1 \mathrm{~min}$, gently probe the surface of the mixture with a finger gloved with an unpowdered, non-water-rinsed latex surgical glove. and observe if libres forni between the cement and the glove as the finger leaves the surlace. Clean the glove of all adherent material.

B.4.3 Repeat the probing process at intervals of $15 \mathrm{~s}$, gently mixing the cement so as to expose a fresh surface for each probing, until the gloved finger separates cleanly from the cement. Record the time at which this first occurs as the doughing time of that mixture.

B.4.4 Repeat B.4.1 to B.4.3 for a second unit of cement.

B.4.5 If the two doughing times differ by more than $30 \mathrm{~s}$, repeat B.4.1 to B.4.3 for a further two units of cement.

\section{B.5 Calculation and expression of results}

Calculate the average doughing time of the two or four determinations made. Round the result to the nearest $15 \mathrm{~s}$ and express this as the average doughing time.

\section{B.6 Test report}

The test report shall include at least the following information:

a) the identity (including batch or lot number) of the cement;

b) the average doughing time:

c) the minimum and maximum doughing times 
$583^{\circ}: 190 ?(5)$

\section{Annex C}

(normative)

Method for determination of maximum temperature and selting time of liquid-power mixture

\section{C.1 Principle}

The exothermic reaction occurring when the powder and liquid components ase mixed is monilsred and the maximum: temperature alla!nen b. "ie bulk is recorded. The selling time is lakivi $4,:$ lime taken to reach a temperature midway $c:$ ": ainbient and maximum.

\section{C.2 Apparatus}

C.2.1 Mould and plunger of dimensir."1s strown in ligure C.1, mide of polytelral roethylene, poly(ethylene 1, ophthalate), poly s... othylne, or high density polyethylene. exii a with a thermocouple of wire diameled roximately $0.5 \mathrm{~mm}$, positic ed with its junction : , $0,5 \mathrm{~mm}$ above the Intelital surface of the mou: hase.

C.2.2 Device capable of converling the thermocouple output signal into temi rature readings and making a continuous record' temperature, the thermocouple and convertim. tevice having an accuracy of $\pm 0.5^{\circ} \mathrm{C}$.

C.2.3 C-clamp or other device for clamping the plunger and mould together.

C.2.4 Timing device of accuracy $\pm 0,2 \mathrm{~s}$.

C.2.5 Equipment as recommended by the cement manufacturet, for mixing the cement.

C.2.6 Thermometer.

\section{C.3 Test conditions}

Maintain the mixing and test equi, $\quad t$ and the contents of the cement unit at $23^{\circ} \mathrm{C} \quad \mathrm{C}$ and at a R.H. of nol less than $40 \%$ for at least $16 \mathrm{~h}$ before beginning the test. Perform the test it $23^{\circ} \mathrm{C} \pm 1{ }^{\circ} \mathrm{C}$ and at a R.H. of not less than $40 \%$

\section{C.4 Procedure}

C.4.1 Recold the ambient temperalure

C.4.2 Mix all the componen?s of a single unlt of cement following the manufacturer's instructions.

C.4.3 Start the timir!n device as soon as the powder and liquid come inte contact.

C.4.4 For cements intended for dough usage, determine when the doug! ing time $r$ the mixture has been reached by mean: -1 the rocedure given in B.4.2 a. 'B.4.3. Within', in a' or this time, gently pack:- oximately $25 \mathrm{y}$ : if cement into the mould, seat ll:: : lunger and trim oll any cement expelled from the mould for coments intended for syringe usage, fill the mould fiom the syringe and proceed as for dough usage cenients.

C.4.5 Continue the temperature measurement until shortly after the tempetalure begins to fall.

C.4.6 Repeat C. 4.2 to C. 4.5 for a second unit of cement.

C.4.7 If the two maximum temperatures (see C.5.1) dil! !y more than $10^{\circ} \mathrm{C}$. $"$ ' the selting times (see C.5.21 tifle by more tlian i in, 1:peat C.4.1 to C.4.5 for ther two units of cument.

\section{C.5 Calculation and expression of results}

\section{C.5.1 Maximuin temperature}

C.5.1.1 For each unit of cement, plot the recorded temperatures against time and record the highest temperature altained to the nearest $1^{\circ} \mathrm{C}$ as the maximum temperature for the sample.

C.5.1.2 Calculate the average value for the two or four determinations. Round the result to the nearest $1^{\circ} \mathrm{C}$ (rounding values of $0,5^{\circ} \mathrm{C}$ upwards) and record this as the maximum temperature.

NOTE 5 An example of a plot is shown in figure C.2. 
(i)

5.2 Setting time

5.2.1 For each unit of cement, determine from the $x$ made in C.5.1 the selting time, 7 ; measured im the beginning of mixing until the temperature

the polymerizing mass reaches

$$
\frac{T_{\text {max }}+T_{\text {amb }}}{2}=7_{\text {amb }}+\left(\frac{T_{\text {max }}-T_{\text {amb }}}{2}\right)
$$

where

$T_{\text {amb }}$ is the recorded ambient temperature (see C.4.1):

$T_{\max }$ is the highest temperature attained.
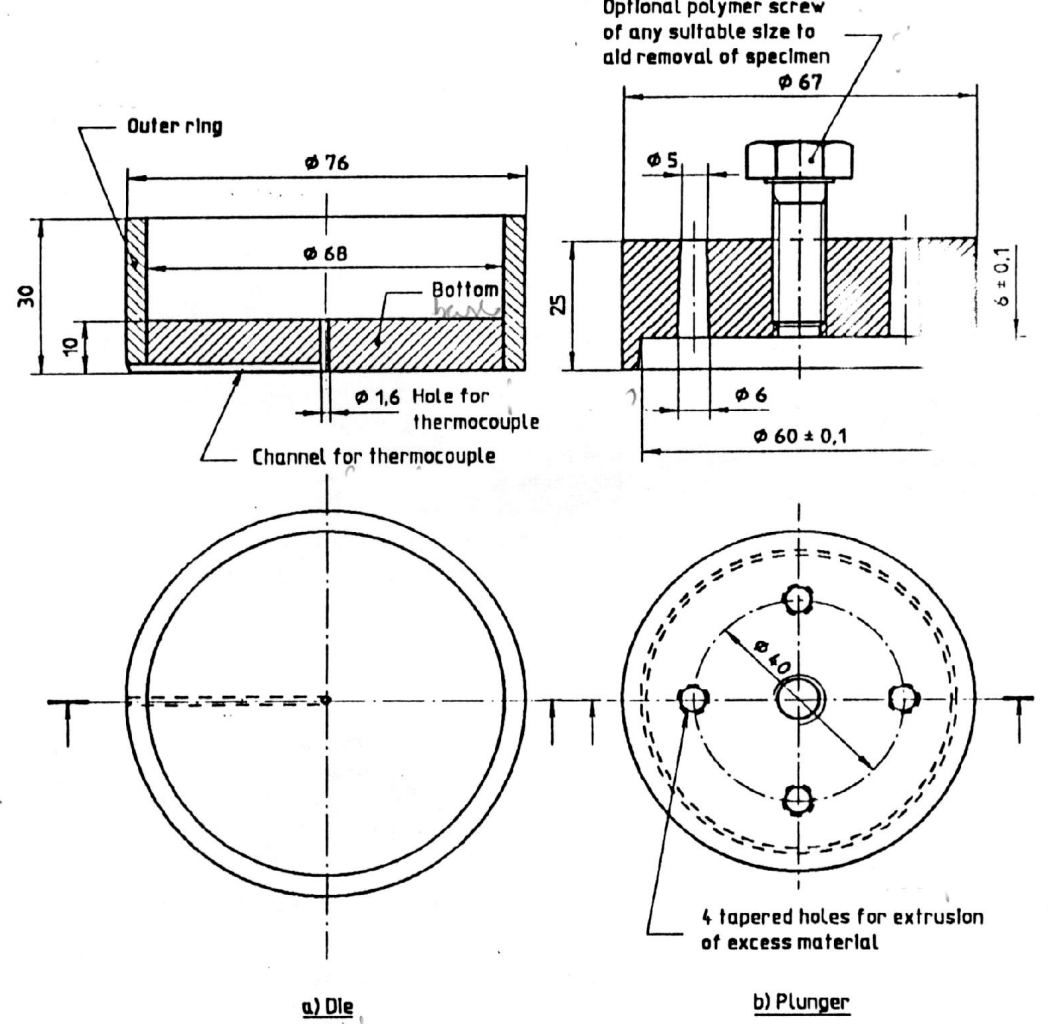

laterial for all components: polytetrafluoroethylene, poly(ethylene terephthalate), polyoxymethylene, or high density olyethylene.

II dimensions $\pm 0,2$ unless otherwise specified.

Figure C.1 - Mould for determination of maximum temperature and setting time 


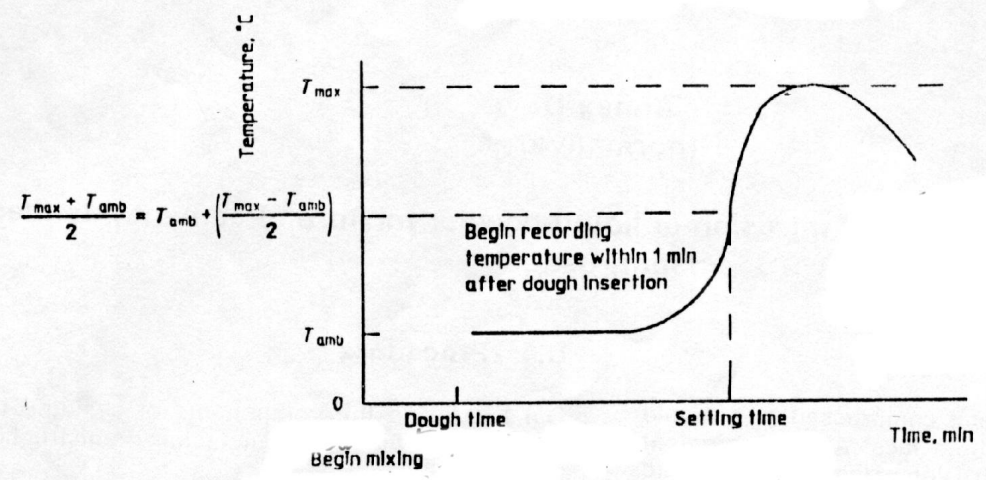

Figure C.2 - Typical curve Ior determination of maximum cumperature and setting time

C.5.2.2 Record the value of $\%$ to the ncarest $5 \mathrm{~s}$. Calculate the average value of 7 for the two or four determinations. Round the result to the nearest $15 \mathrm{~s}$, and express this as the setting time

\section{C.6 Test report}

The test report shall include at least the following information: a) the identity (including batch or lot numbers) of the cement;

b) the average maximum temperature;

c) the individual maximum temperalures;

d) the average setting time:

e) the individual setting times 


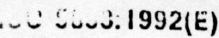

Annex D

(normative)

Method for determination of intrusion of liquid-powder mixture of cement intended for dough usage

\section{D.1 Principle}

The cement is mixed and compressed in a mould having a perforated bottom face. After the cement has set, the extent of intrusion of the cement into the perforations is measured.

\section{D.2 Apparatus}

D.2.1 Mould and plunger of dimensions shown in figure D.1. made of polytetrafluoroethylene, poly(ethylene terephthalate), polyoxymethylene or high density polyethylene.

D.2.2 Means of applying a compressive force to the mould.

D.2.3 Means of measuring the extent of intrusion to an accuracy of $\pm 0,5 \mathrm{~mm}$.

D.2.4 Equipment as recommended by the cement manufacturer, for mixing the cement.

\section{D.3 Test conditions}

Maintain the mixing and test equipment and the contents of the cement units at $23^{\circ} \mathrm{C} \pm 1^{\circ} \mathrm{C}$. for at least $16 \mathrm{~h}$ before beginning the test. Perform the test at $23^{\circ} \mathrm{C} \pm 1{ }^{\circ} \mathrm{C}$.

\section{D.4 Procedure}

D.4.1 Mix all the components of a single unit of cement, following the manulacturer's instructions.

D.4.2 By means of the procedure given in B.4.2 and B.4.3, determine when the doughing time of the mixiure has been reachied. Immediately pack the mixture gently into the mould and insert the plunger.

D.4.3 $1 \mathrm{~min} \pm 10 \mathrm{~s}$ after doughing time was reached, apply a force of $49 \mathrm{~N}$ to the plunger for a period of $1 \mathrm{~min} \pm 2 \mathrm{~s}$. Remove the lorce and allow the cement to set.

D.4.4 Remove the set cement from the mould and measure the extent of intrusion of the cement into each of the four holes in the mould Calculate the average of the four values to the nearest $0.5 \mathrm{~mm}$.

D.4.5 If the average intrusion is less tharr $2 \mathrm{~mm}$, repeat D.4.1 to D.4.4 for a second unit of cement.

\section{D.5 Test report}

The test report shall include at least the following information:

a) the identity (including batch or lot numbers) of the cement;

b) the average intrusion for each sample tested. 
$1=\cdots \cdots 99.1009(5)$

Dimensions in millimetres

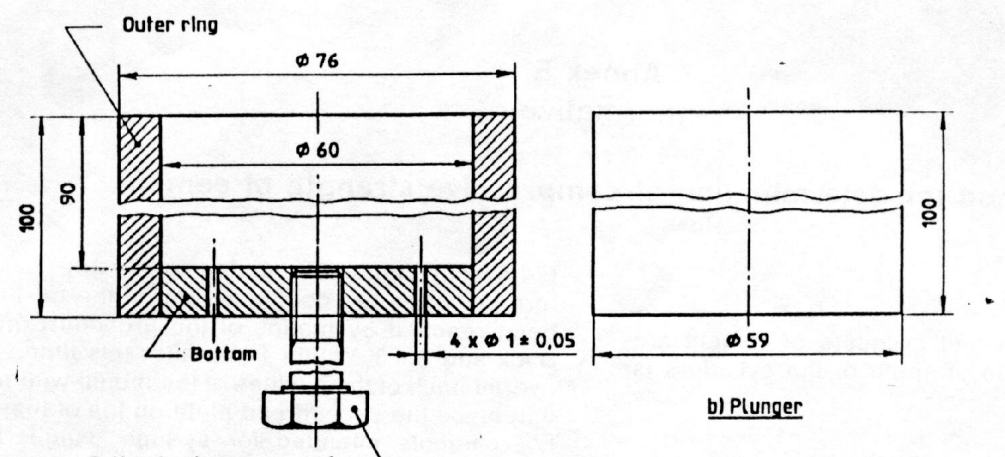

Optlonal polymer screw of

any sultable slze to ald

removal of test specimen

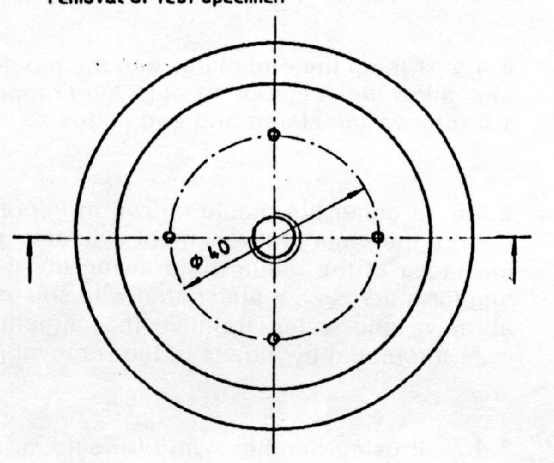

a) Dle

Tolerances on all dimencions: \pm 0.2 unless otherwise specified.

Matérial for all components: polyletrafluoroethylene. poly(ethylene teraphthalate), polyoxymethylene or high density polyethylene.

Figure D.1 - Mould for determination of Intrusion 


\section{Annex $\mathrm{E}$ \\ (normative)}

\section{Method for determination of compressive strength of cement}

\section{E.1 Principle}

The cement is mixed and cylinders of cement are cast. The compressive strength of the cylinders is then determined.

\section{E.2 Apparalus}

E.2.1 Mould, end plates and removal rod of dimensions shown in figure E.1, made of stainless steel, or other device for producing cylinders of cement of appropriate dimensions.

E.2.2 C-clamp or other device for clamping the mould and end plates together.

E.2.3 240-mesh silicon carbide grinding abrasive and a flat plate.

E.2.4 Mould release agent (optional).

E.2.5 Equipment as recommended by the cement manulaclurer, for mixing the cement.

E.2.6 Test machine capable of applying and measuring a compressive force of a least $4 \mathrm{kN}$, equipped to record load versus deformation.

\section{E.3 Test conditions}

Maintain the mixing and test equipment at $23^{\circ} \mathrm{C} \pm 1^{\circ} \mathrm{C}$, for at least $16 \mathrm{~h}$ belore beginning the test. Perform the test at $23^{\circ} \mathrm{C}+1^{\circ} \mathrm{C}$

\section{E.4 Procedure}

E.4.1 If required, lightly coat the interior faces of the mould and the Inward faces of the end plates with mould release agent.

E.4.2 Place the mould on one end plate.

E.4.3 Mix all the components of a single unit of cement, following the manufacturer's instructions.
E.4.4 For cements intended for dough usage, determine when the doughing time of the mrixture has been reached by means of the procedure given in B.4.2 and B.4.3. Within $1 \mathrm{~min}$ after this time, slightly overfill each of the cavities of the mould with mixture and place the second end plate on top of the mould. For cements intended for syringe usage, fill the cavities from the syringe and proceed as for dough usage cements.

E.4.5 Clamp the end plates and the mould together and allow the cement to set. After approximately $1 \mathrm{~h}$ remove the clamp and end plates.

E.4.6 If using the mould shown in figure E.1. grind both of the ends of the cement cylinders plane with the faces of the mould by drawing the mould back and forth across a plate coated with silicone carbide abrasive and water. Remove the cement cylinders from the mould by means of the removal rod.

E.4.7 If using another type of mould, grind the cement cylinders so as to produce right cylinders of length $12 \mathrm{~mm} \pm 0.1 \mathrm{~mm}$ and diameter $6 \mathrm{~mm}$ $\pm 0,1 \mathrm{~mm}$.

E.4.8 Maintain the cylinders at $23^{\circ} \mathrm{C} \pm 1{ }^{\circ} \mathrm{C}$.

E.4.9 At a time $24 \mathrm{~h} \pm 2 \mathrm{~h}$ after the mixing of the cement was begun. measure the average diameter of each test piece, taking the measurements in two perpendicular directions at at least two sections. Place a cylinder in the test machine, without any type of pad between the cylinder and the platen of the lest machine. Operate the lest machine to produce a curve of deformation against load, using a constant cross-head speed in the range $20 \mathrm{~mm} / \mathrm{min}$ to $25.4 \mathrm{~mm} / \mathrm{min}$. Stop the machine when the cylinder fraclures or when the upper yield point has been passed.

NOTE 6 An example of an idealized load deformation curve is shown in figure E. 2 .

E.4.10 Repeat E.4.9 for each of the cylinders. 
E.5 Calculation and expression of results

For each cylinder, record the force applied to cause fracture, or the $2 \%$ offset load or the upper yield point load whichever occurred first. Divide this force by the original cross-sectional area of the cylinder and express the quntient as the compressive strength in megapas $\mathrm{c} / \mathrm{s}$. Calculate the average compressive strength. the five cylinders.

\section{E.6 Test report}

The test report shall include at least the following information:

a) identity (including batch or lot numbers) of the cement;

b) the average compressive strength in megapascals and the standard deviation.
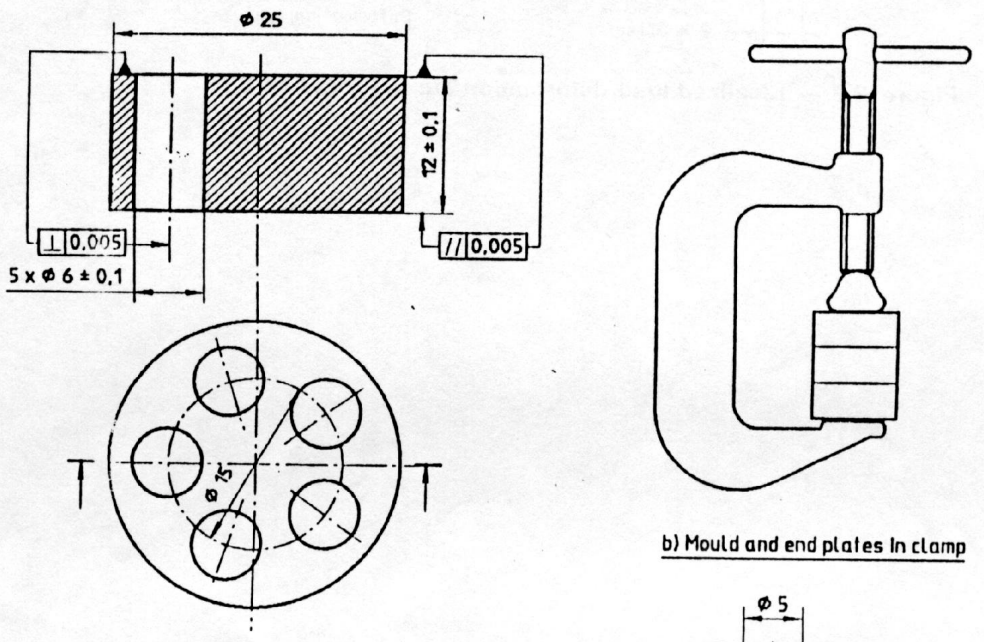

b) Mould and end plates in clamp

a) Perforated plate
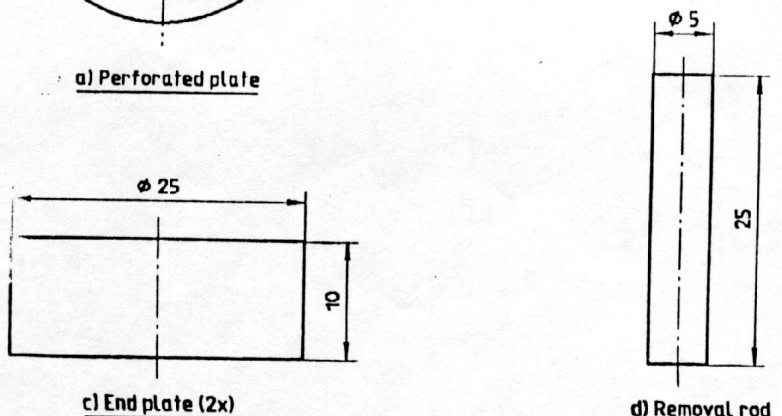

Tolerance on all dimensions: $\pm 0,2$ unless otherwise specified

Figure E.1 - Mould for preparing compressive strength test specimens 
SO 5833:1992(E)

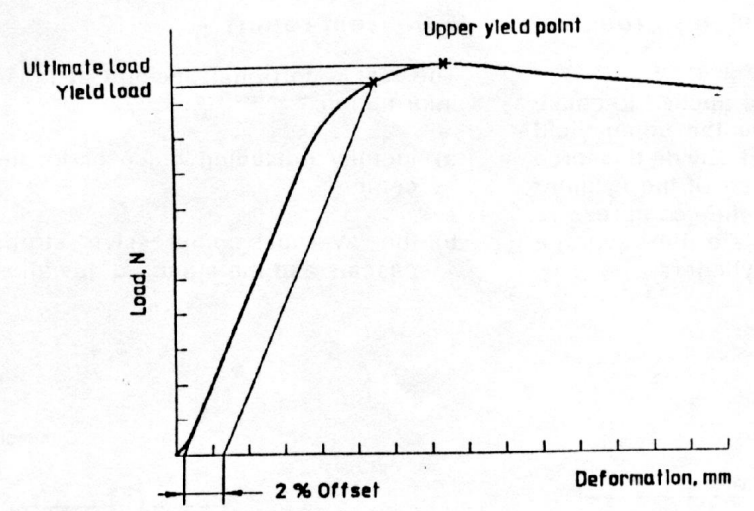

Figure E.2 = Idealized load/deformation curve for cement 
iso 5833:1 Uง (E)

\section{Annex $\mathbf{F}$}

(normative)

\section{Method for determination of bending modulus and bending strength of cement}

\section{F.1 Principle}

The cement is mixed and test strips are prepared. The bending modulus and bending strength of the strips are determined by means of a lour-point bend test.

\section{F.2 Apparatus}

F.2.1 Bend test machine having a cross-head speed of $5 \mathrm{~mm} / \mathrm{min} \pm 1 \mathrm{~mm} / \mathrm{min}$, equipped with a device for measuring and recording the deflection of the centre of the specimen to an accuracy of $\pm 0,05 \mathrm{~mm}$.

F.2.2 Four-point bend test rig having the dimensions shown in figure F.1, with means to preven misalignment of the test specimen on the supports.

F.2.3 Water bath capable of being controlled at $37^{\circ} \mathrm{C} \pm 1{ }^{\circ} \mathrm{C}$.

F.2.4 Six moulds made of polytetrafluoroethylene, poly(ethylene terephthalate), polyoxymethylene or high densily polyethylene, having a cavity of approximalely $75 \mathrm{~mm}$ length. $10 \mathrm{~mm}$ width and $3.3 \mathrm{~mm}$ depth, or one mould having six such cavities, or one mould of $75 \mathrm{~mm}$ length, $90 \mathrm{~mm}$ width and $3,3 \mathrm{~mm}$ depth

F.2.5 Flat, smooth plates (two for each mould) made of the materials listed in F.2.4, of size sulficient to cover completely the upper and lower surfaces of the mould(s) described in F.2.4.

\section{F.2.6 Polyester film.}

F.2.7 C-clamp(s) or other device(s) for clamping the mould(s) between the top and boltom plates.

F.2.8 Equipment as recommended by the cement manufacturer, for mixing cement.

\section{F.3 Test condition:}

Maintain the mould(s), "'ates, mixing equipment and the contents of the cement unlt at $23^{\circ} \mathrm{C} \pm 1{ }^{\circ} \mathrm{C}$ for at least $16 \mathrm{~h}$ before casling the test strip(s). Cast the test strip(s) at $23^{\circ} \mathrm{C} \pm 1^{\circ} \mathrm{C}$

\section{F.4 Procedure}

F.4.1 Cover the bottom plate(s) of the mould(s) with polyester film. Place the mould(s) on top of the plate(s).

F.4.2 Mix all the components of a single unit of cement, following the manufacturer's instructions.

F.4.3 For cements intended for dough usage, delermine when the doughing time of the mixture has been reached by means of the procedure given in B.4.2 and B.4.3. Within $1 \mathrm{~min}$ after this time, gently pack the mixture into the mould(s), add a layer of polyester film, add the top plate(s) and clamp the top and bottom plates to the mould(s). For cements intended for syringe use, fill the moulds from the syringe and proceed as for dough usage cements.

F.4.4 After approximalely $1 \mathrm{~h}$ remove the clamp, the top and bottom plates and the polyester film.

F.4.5 If a single large strip has been produced, prepare test strip $75 \mathrm{~mm}$ long and $10 \mathrm{~mm}$ wide from the large strip by sawing length-wise, using a diamond blade and water cooling.

Take care to avoid over-heating the test strips. Wet grind the edges and top faces of the strips with $\mathbf{4 0 0}$ grade emery paper to the required breadth and thickness. Denote the unground bottom face as it is to be used as the tensile face during bending.

If single test strips are prepared in individual mould cavities, remove the strips from their moulds. Immerse all strips in water at $37^{\circ} \mathrm{C} \pm 1^{\circ} \mathrm{C}$ for $50 \mathrm{~h} \pm 2 \mathrm{~h}$.

F.4.6 Remove one test strip from the water bath, measure its thickness and width to an accuracy of $\pm 0,1 \mathrm{~mm}$ taking readings at at least three crosssections of the strip and place it symmetrically in the four-point bend test rig.

F.4.7 By means of the bend test machine, immediately increase the force on the central loading plunger from zero using a cross-head speed of $5 \mathrm{~mm} / \mathrm{min} \pm 1 \mathrm{~mm} / \mathrm{min}$, recording the deflection of the strip as a function of the applied force. Continue to increase the force until the test strip breaks. 
4.8 Record the deflection occurring at applied rces of $15 \mathrm{~N}$ and $50 \mathrm{~N}$ to the nearest $0,05 \mathrm{~mm}$. fecord the force at break to the nearest $0,5 \mathrm{~N}$.

:4.9 Repeat F 4.6 to F.4.8 for each of the five renaining test strips.

\section{$: .5$ Calculation and expression of results}

\section{:.5.1 Bending modulus}

or each test strip, calculate the bending modulus, $\therefore$ in megapascals, from the expression:

$$
E=\frac{\Delta F a}{4 J h^{3}} \times\left(3 l^{2}-4 a^{2}\right)
$$

there $f \quad$ is the difference between the deflections under the loads of $15 \mathrm{~N}$ and $50 \mathrm{~N}$, in millimetres;

$b$ is the average measured width of strip in millimetres;

$h$ is the average measured thickness of strip in millimetres;

$l$ is the distance between outer loading points $(60 \mathrm{~mm})$;

$\Delta F \quad$ is the load range $(50 \mathrm{~N}-15 \mathrm{~N}=35 \mathrm{~N})$;

is the distance between the inner and outer loading points $(20 \mathrm{~mm})$

Calculate the average value of bending modulus for the six test specimens expressed in megapascals and the standard deviation.

Dimensions in millimetres

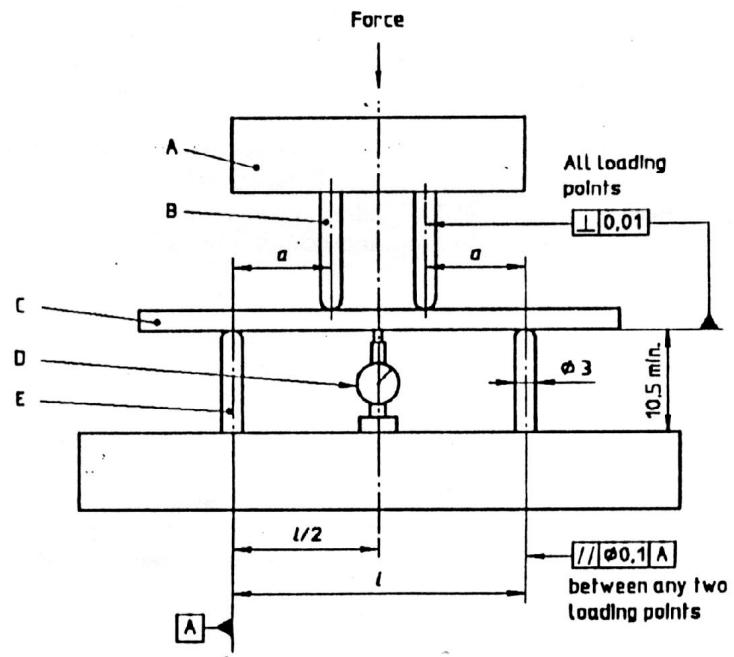

A - Central loading plunger

B - Inner loading points

C - Test strip

D - Device for measuring deflection (dial gauge or any other device)

E - Outer loading points

$I$ is the distance between outer loading points $(60 \mathrm{~mm})$ -

$a$ is the distance between outer and inner loading points $(20 \mathrm{~mm} \pm 1 \mathrm{~mm})-$

Figure F.1 - Four-point bend test rig 


\section{F.5.2 Bending strength}

For each test strip, calculate the bending strength, $B$, in megapascals, from the expression:

$$
B=\frac{3 F a}{b h^{2}}
$$

where

$F \quad$ is the force at break in newtons:

$b$ is the average measured width of strip in millimetres:

$h \quad$ is the average measured thickness of strip in millimetres;

a is the distance between the inner and outer loading points $(20 \mathrm{~mm})$.
Calculate the average value of the bending strength for the slx test specimens expressed in megapascals and the standard deviation.

\section{F.6 Test report}

The test report shall include at least the following information:

a) identity (including batch or lot numbers) of the cement:

b) the average of the values of bending modulus for the six test specimens expressed in megapascals and the standard deviation:

c) the average of the values of bending strength for the six test specimens expressed in megapascals and the standard deviation. 
ANEXO C

FICHA DE DADOS DOS CORPOS DE PROVA 


\section{IDENTIFICAÇÃO}

Número corpinho:

Número partida:

Marca do cimento:

Data fabricação:

Número lote:

Tipo de cimento:

Esterilização:

Data do teste:

\section{MEDIDAS}

\begin{tabular}{|c|c|c|c|}
\hline \multirow{2}{*}{ Altura: } & \multicolumn{3}{|c|}{ Diâmetro } \\
\hline & & \multicolumn{2}{|r|}{0} \\
\hline \multicolumn{4}{|l|}{ INSPEÇÃO VISUAL } \\
\hline Presença bolhas: & & \multicolumn{2}{|l|}{ Número bolhas: } \\
\hline \multicolumn{4}{|l|}{ Localização bolhas: } \\
\hline \multicolumn{4}{|l|}{ PADRONIZAÇÃO } \\
\hline \multicolumn{2}{|l|}{ Pré-carga: 200 kg (5 kg) } & \multicolumn{2}{|c|}{ Tempo acomodação: 30 segundos } \\
\hline \multicolumn{2}{|l|}{ Célula de carga: 500 kg } & $2^{\mathrm{a}}$ Marcha & Velocidade $=0,1$ \\
\hline Deformação (mm) & Valor lido & (C. aplic/40)*9,8 & Tensão= F/A \\
\hline 0,00 & & & \\
\hline 0,05 & & & \\
\hline 0,10 & & & \\
\hline 0,15 & & & \\
\hline 0,20 & & & \\
\hline 0,25 & & & \\
\hline 0,30 & & & \\
\hline 0,35 & & & \\
\hline 0,40 & & & \\
\hline 0,45 & & & \\
\hline 0,50 & & & \\
\hline 0,55 & & & \\
\hline 0,60 & & & \\
\hline 0,65 & & & \\
\hline 0,70 & & & \\
\hline 0,75 & & & \\
\hline 0,80 & & & \\
\hline 0,85 & & & \\
\hline 0,90 & & & \\
\hline 0,95 & & & \\
\hline 1,00 & & & \\
\hline 1,05 & & & \\
\hline 1,10 & & & \\
\hline
\end{tabular}


ANEXO D

DADOS DOS CORPOS DE PROVA DO GRUPO 1M 


\begin{tabular}{|c|c|c|c|c|c|c|}
\hline & 1 & 2 & 3 & 4 & 5 & 6 \\
\hline 0,00 & $1,49 \mathrm{E}+00$ & $1,63 \mathrm{E}+00$ & $1,61 \mathrm{E}+00$ & $1,39 \mathrm{E}+06$ & $1,51 \mathrm{E}+06$ & $1,54 \mathrm{E}+06$ \\
\hline 0,05 & $3,79 \mathrm{E}+00$ & $6,06 \mathrm{E}+00$ & $9,62 \mathrm{E}+00$ & $4,43 \mathrm{E}+06$ & $4,93 \mathrm{E}+06$ & $4,76 \mathrm{E}+06$ \\
\hline 0,10 & $7,18 \mathrm{E}+00$ & $1,09 \mathrm{E}+01$ & $1,60 \mathrm{E}+01$ & $8,70 \mathrm{E}+06$ & $9,31 \mathrm{E}+06$ & $9,32 \mathrm{E}+06$ \\
\hline 0,15 & $1,17 \mathrm{E}+01$ & $1,81 \mathrm{E}+01$ & $2,27 \mathrm{E}+01$ & $1,46 \mathrm{E}+07$ & $1,54 \mathrm{E}+07$ & $1,60 \mathrm{E}+07$ \\
\hline 0,20 & $1,65 \mathrm{E}+01$ & $2,38 \mathrm{E}+01$ & $2,95 \mathrm{E}+01$ & $2,02 \mathrm{E}+07$ & $2,13 \mathrm{E}+07$ & $2,24 \mathrm{E}+07$ \\
\hline 0,25 & $2,22 \mathrm{E}+01$ & $2,92 \mathrm{E}+01$ & $3,55 \mathrm{E}+01$ & $2,61 \mathrm{E}+07$ & $2,75 \mathrm{E}+07$ & $2,93 \mathrm{E}+07$ \\
\hline 0,30 & $2,74 \mathrm{E}+01$ & $3,58 \mathrm{E}+01$ & $4,03 \mathrm{E}+01$ & $3,10 \mathrm{E}+07$ & $3,29 \mathrm{E}+07$ & $3,50 \mathrm{E}+07$ \\
\hline 0,35 & $3,23 \mathrm{E}+01$ & $4,14 \mathrm{E}+01$ & $4,48 \mathrm{E}+01$ & $3,70 \mathrm{E}+07$ & $3,69 \mathrm{E}+07$ & $4,03 \mathrm{E}+07$ \\
\hline 0,40 & $3,66 \mathrm{E}+01$ & $4,60 \mathrm{E}+01$ & $4,85 \mathrm{E}+01$ & $4,20 \mathrm{E}+07$ & $4,11 \mathrm{E}+07$ & $4,41 \mathrm{E}+07$ \\
\hline 0,45 & $4,15 \mathrm{E}+01$ & $4,91 \mathrm{E}+01$ & $5,10 \mathrm{E}+01$ & $4,72 \mathrm{E}+07$ & $4,46 \mathrm{E}+07$ & $4,72 \mathrm{E}+07$ \\
\hline 0,50 & $4,59 \mathrm{E}+01$ & $5,13 \mathrm{E}+01$ & $5,25 \mathrm{E}+01$ & $5,08 \mathrm{E}+07$ & $4,70 \mathrm{E}+07$ & $4,96 \mathrm{E}+07$ \\
\hline 0,55 & $4,88 \mathrm{E}+01$ & $5,26 \mathrm{E}+01$ & $5,32 \mathrm{E}+01$ & $5,43 \mathrm{E}+07$ & $4,83 \mathrm{E}+07$ & $5,18 \mathrm{E}+07$ \\
\hline 0,60 & $5,15 \mathrm{E}+01$ & $5,33 \mathrm{E}+01$ & $5,35 \mathrm{E}+01$ & $5,67 \mathrm{E}+07$ & $4,90 \mathrm{E}+07$ & $5,31 \mathrm{E}+07$ \\
\hline 0,65 & $5,32 \mathrm{E}+01$ & $5,35 \mathrm{E}+01$ & $5,36 \mathrm{E}+01$ & $5,82 \mathrm{E}+07$ & $4,95 \mathrm{E}+07$ & $5,39 \mathrm{E}+07$ \\
\hline 0,70 & $5,45 \mathrm{E}+01$ & $5,36 \mathrm{E}+01$ & $5,35 \mathrm{E}+01$ & $5,96 \mathrm{E}+07$ & $4,97 \mathrm{E}+07$ & $5,45 \mathrm{E}+07$ \\
\hline 0,75 & $5,53 \mathrm{E}+01$ & $5,34 \mathrm{E}+01$ & $5,34 \mathrm{E}+01$ & $6,02 \mathrm{E}+07$ & $4,99 \mathrm{E}+07$ & $5,48 \mathrm{E}+07$ \\
\hline 0,80 & $5,59 \mathrm{E}+01$ & $5,33 \mathrm{E}+01$ & $5,33 \mathrm{E}+01$ & $6,04 \mathrm{E}+07$ & $4,99 \mathrm{E}+07$ & $5,51 \mathrm{E}+07$ \\
\hline 0,85 & $5,62 \mathrm{E}+01$ & $5,33 \mathrm{E}+01$ & $5,32 \mathrm{E}+01$ & $6,05 \mathrm{E}+07$ & $4,99 \mathrm{E}+07$ & $5,53 \mathrm{E}+07$ \\
\hline 0,90 & $5,63 \mathrm{E}+01$ & $5,33 \mathrm{E}+01$ & $5,32 \mathrm{E}+01$ & $6,04 \mathrm{E}+07$ & $4,99 \mathrm{E}+07$ & $5,55 \mathrm{E}+07$ \\
\hline 0,95 & $5,65 \mathrm{E}+01$ & $5,32 \mathrm{E}+01$ & $5,33 \mathrm{E}+01$ & $6,04 \mathrm{E}+07$ & $4,99 \mathrm{E}+07$ & $5,56 \mathrm{E}+07$ \\
\hline 1,00 & $5,67 \mathrm{E}+01$ & $5,32 \mathrm{E}+01$ & $5,34 \mathrm{E}+01$ & $6,01 \mathrm{E}+07$ & $5,00 \mathrm{E}+07$ & $5,57 \mathrm{E}+07$ \\
\hline 1,05 & $5,69 \mathrm{E}+01$ & & $5,36 \mathrm{E}+01$ & $6,00 \mathrm{E}+07$ & $5,01 \mathrm{E}+07$ & $5,58 \mathrm{E}+07$ \\
\hline 1,10 & $5,73 \mathrm{E}+01$ & & $5,38 \mathrm{E}+01$ & & & \\
\hline 1,15 & & & & & & \\
\hline 1,20 & & & & & & \\
\hline 1,25 & & & & & & \\
\hline
\end{tabular}

Continua 
Continuação do Anexo D

\begin{tabular}{|c|c|c|c|c|c|}
\hline 7 & 8 & 9 & 10 & 11 & 12 \\
\hline $1,48 \mathrm{E}+06$ & $1,66 \mathrm{E}+06$ & $1,64 \mathrm{E}+06$ & $1,54 \mathrm{E}+06$ & $1,62 \mathrm{E}+06$ & $1,55 \mathrm{E}+06$ \\
\hline $4,32 \mathrm{E}+06$ & $6,15 \mathrm{E}+06$ & $1,20 \mathrm{E}+07$ & $5,75 \mathrm{E}+06$ & $4,24 \mathrm{E}+06$ & $5,68 \mathrm{E}+06$ \\
\hline $8,77 \mathrm{E}+06$ & $1,12 \mathrm{E}+07$ & $1,65 \mathrm{E}+07$ & $9,28 \mathrm{E}+06$ & $8,00 \mathrm{E}+06$ & $1,07 \mathrm{E}+07$ \\
\hline $1,62 \mathrm{E}+07$ & $1,77 \mathrm{E}+07$ & $2,22 \mathrm{E}+07$ & $1,26 \mathrm{E}+07$ & $1,41 \mathrm{E}+07$ & $1,68 \mathrm{E}+07$ \\
\hline $2,14 \mathrm{E}+07$ & $2,46 \mathrm{E}+07$ & $2,86 \mathrm{E}+07$ & $1,84 \mathrm{E}+07$ & $1,96 \mathrm{E}+07$ & $2,34 \mathrm{E}+07$ \\
\hline $2,82 \mathrm{E}+07$ & $3,06 \mathrm{E}+07$ & $3,32 \mathrm{E}+07$ & $2,51 \mathrm{E}+07$ & $2,58 \mathrm{E}+07$ & $2,99 \mathrm{E}+07$ \\
\hline $3,36 \mathrm{E}+07$ & $3,63 \mathrm{E}+07$ & $3,76 \mathrm{E}+07$ & $3,13 \mathrm{E}+07$ & $3,09 \mathrm{E}+07$ & $3,57 \mathrm{E}+07$ \\
\hline $3,88 \mathrm{E}+07$ & $4,36 \mathrm{E}+07$ & $4,14 \mathrm{E}+07$ & $3,66 \mathrm{E}+07$ & $3,68 \mathrm{E}+07$ & $4,13 \mathrm{E}+07$ \\
\hline $4,30 \mathrm{E}+07$ & $4,69 \mathrm{E}+07$ & $4,45 \mathrm{E}+07$ & $4,12 \mathrm{E}+07$ & $4,13 \mathrm{E}+07$ & $4,62 \mathrm{E}+07$ \\
\hline $4,72 \mathrm{E}+07$ & $5,21 \mathrm{E}+07$ & $4,70 \mathrm{E}+07$ & $4,65 \mathrm{E}+07$ & $4,45 \mathrm{E}+07$ & $5,06 \mathrm{E}+07$ \\
\hline $5,06 \mathrm{E}+07$ & $5,41 \mathrm{E}+07$ & $4,85 \mathrm{E}+07$ & $5,09 \mathrm{E}+07$ & $4,92 \mathrm{E}+07$ & $5,38 \mathrm{E}+07$ \\
\hline $5,31 \mathrm{E}+07$ & $5,53 \mathrm{E}+07$ & $4,92 \mathrm{E}+07$ & $5,42 \mathrm{E}+07$ & $5,21 \mathrm{E}+07$ & $5,59 \mathrm{E}+07$ \\
\hline $5,45 \mathrm{E}+07$ & $5,60 \mathrm{E}+07$ & $4,96 \mathrm{E}+07$ & $5,66 \mathrm{E}+07$ & $5,44 \mathrm{E}+07$ & $5,72 \mathrm{E}+07$ \\
\hline $5,58 \mathrm{E}+07$ & $5,63 \mathrm{E}+07$ & $4,97 \mathrm{E}+07$ & $5,81 \mathrm{E}+07$ & $5,56 \mathrm{E}+07$ & $5,80 \mathrm{E}+07$ \\
\hline $5,67 \mathrm{E}+07$ & $5,63 \mathrm{E}+07$ & $4,99 \mathrm{E}+07$ & $5,88 \mathrm{E}+07$ & $5,63 \mathrm{E}+07$ & $5,75 \mathrm{E}+07$ \\
\hline $5,73 \mathrm{E}+07$ & $5,63 \mathrm{E}+07$ & $5,02 \mathrm{E}+07$ & $5,91 \mathrm{E}+07$ & $5,67 \mathrm{E}+07$ & $5,83 \mathrm{E}+07$ \\
\hline $5,79 \mathrm{E}+07$ & $5,63 \mathrm{E}+07$ & $5,06 \mathrm{E}+07$ & $5,91 \mathrm{E}+07$ & $5,70 \mathrm{E}+07$ & $5,81 \mathrm{E}+07$ \\
\hline $5,83 \mathrm{E}+07$ & $5,62 \mathrm{E}+07$ & $5,09 \mathrm{E}+07$ & $5,89 \mathrm{E}+07$ & $5,71 \mathrm{E}+07$ & $5,79 \mathrm{E}+07$ \\
\hline $5,85 \mathrm{E}+07$ & $5,63 \mathrm{E}+07$ & $5,12 \mathrm{E}+07$ & $5,88 \mathrm{E}+07$ & $5,70 \mathrm{E}+07$ & $5,78 \mathrm{E}+07$ \\
\hline $5,89 \mathrm{E}+07$ & $5,64 \mathrm{E}+07$ & $5,17 \mathrm{E}+07$ & $5,87 \mathrm{E}+07$ & $5,69 \mathrm{E}+07$ & $5,77 \mathrm{E}+07$ \\
\hline $5,92 \mathrm{E}+07$ & & $5,20 \mathrm{E}+07$ & $5,88 \mathrm{E}+07$ & $5,70 \mathrm{E}+07$ & $5,75 \mathrm{E}+07$ \\
\hline $5,96 \mathrm{E}+07$ & & $5,25 \mathrm{E}+07$ & $5,89 \mathrm{E}+07$ & & $5,76 \mathrm{E}+07$ \\
\hline $6,00 \mathrm{E}+07$ & & $5,29 \mathrm{E}+07$ & & & $5,77 \mathrm{E}+07$ \\
\hline & & $5,34 \mathrm{E}+07$ & & & \\
\hline & & $5,38 \mathrm{E}+07$ & & & \\
\hline & & $5,42 \mathrm{E}+07$ & & & \\
\hline
\end{tabular}

Continua 
Continuação do Anexo D

\begin{tabular}{|c|c|c|c|c|c|}
\hline 13 & 14 & 15 & 16 & 17 & 18 \\
\hline $1,55 \mathrm{E}+06$ & $1,68 \mathrm{E}+06$ & $1,62 \mathrm{E}+06$ & $1,43 \mathrm{E}+06$ & $1,48 \mathrm{E}+06$ & $1,33 \mathrm{E}+06$ \\
\hline $4,92 \mathrm{E}+06$ & $5,15 \mathrm{E}+06$ & $3,70 \mathrm{E}+06$ & $5,83 \mathrm{E}+06$ & $3,92 \mathrm{E}+06$ & $3,37 \mathrm{E}+06$ \\
\hline $8,95 \mathrm{E}+06$ & $1,02 \mathrm{E}+07$ & $7,22 \mathrm{E}+06$ & $9,06 \mathrm{E}+06$ & $5,95 \mathrm{E}+06$ & $6,33 \mathrm{E}+06$ \\
\hline $1,40 \mathrm{E}+07$ & $1,61 \mathrm{E}+07$ & $1,17 \mathrm{E}+07$ & $1,45 \mathrm{E}+07$ & $1,01 \mathrm{E}+07$ & $1,00 \mathrm{E}+07$ \\
\hline $1,95 \mathrm{E}+07$ & $2,14 \mathrm{E}+07$ & $1,62 \mathrm{E}+07$ & $1,98 \mathrm{E}+07$ & $1,56 \mathrm{E}+07$ & $1,41 \mathrm{E}+07$ \\
\hline $2,53 \mathrm{E}+07$ & $2,79 \mathrm{E}+07$ & $2,13 \mathrm{E}+07$ & $2,62 \mathrm{E}+07$ & $2,15 \mathrm{E}+07$ & $1,92 \mathrm{E}+07$ \\
\hline $3,09 \mathrm{E}+07$ & $3,33 \mathrm{E}+07$ & $2,58 \mathrm{E}+07$ & $3,28 \mathrm{E}+07$ & $2,74 \mathrm{E}+07$ & $2,46 \mathrm{E}+07$ \\
\hline $3,73 \mathrm{E}+07$ & $3,77 \mathrm{E}+07$ & $3,10 \mathrm{E}+07$ & $3,66 \mathrm{E}+07$ & $3,27 \mathrm{E}+07$ & $2,99 \mathrm{E}+07$ \\
\hline $4,34 \mathrm{E}+07$ & $4,31 \mathrm{E}+07$ & $3,53 \mathrm{E}+07$ & $4,03 \mathrm{E}+07$ & $3,85 \mathrm{E}+07$ & $3,42 \mathrm{E}+07$ \\
\hline $4,83 \mathrm{E}+07$ & $4,69 \mathrm{E}+07$ & $4,03 \mathrm{E}+07$ & $4,40 \mathrm{E}+07$ & $4,33 \mathrm{E}+07$ & $3,87 \mathrm{E}+07$ \\
\hline $5,21 \mathrm{E}+07$ & $4,99 \mathrm{E}+07$ & $4,47 \mathrm{E}+07$ & $4,65 \mathrm{E}+07$ & $4,76 \mathrm{E}+07$ & $4,19 \mathrm{E}+07$ \\
\hline $5,53 \mathrm{E}+07$ & $5,20 \mathrm{E}+07$ & $4,81 \mathrm{E}+07$ & $4,79 \mathrm{E}+07$ & $5,16 \mathrm{E}+07$ & $4,49 \mathrm{E}+07$ \\
\hline $5,73 \mathrm{E}+07$ & $5,35 \mathrm{E}+07$ & $5,14 \mathrm{E}+07$ & $4,84 \mathrm{E}+07$ & $5,52 \mathrm{E}+07$ & $4,74 \mathrm{E}+07$ \\
\hline $5,86 \mathrm{E}+07$ & $5,44 \mathrm{E}+07$ & $5,35 \mathrm{E}+07$ & $4,83 \mathrm{E}+07$ & $5,78 \mathrm{E}+07$ & $4,91 \mathrm{E}+07$ \\
\hline $5,95 \mathrm{E}+07$ & $5,50 \mathrm{E}+07$ & $5,49 \mathrm{E}+07$ & $4,80 \mathrm{E}+07$ & $5,94 \mathrm{E}+07$ & $5,03 \mathrm{E}+07$ \\
\hline $5,99 \mathrm{E}+07$ & $5,53 \mathrm{E}+07$ & $5,58 \mathrm{E}+07$ & $4,77 \mathrm{E}+07$ & $6,02 \mathrm{E}+07$ & $5,14 \mathrm{E}+07$ \\
\hline $5,99 \mathrm{E}+07$ & $5,55 \mathrm{E}+07$ & $5,63 \mathrm{E}+07$ & $4,75 \mathrm{E}+07$ & $6,06 \mathrm{E}+07$ & $5,22 \mathrm{E}+07$ \\
\hline $5,98 \mathrm{E}+07$ & $5,56 \mathrm{E}+07$ & $5,65 \mathrm{E}+07$ & $4,73 \mathrm{E}+07$ & $6,08 \mathrm{E}+07$ & $5,27 \mathrm{E}+07$ \\
\hline $5,97 \mathrm{E}+07$ & $5,57 \mathrm{E}+07$ & $5,65 \mathrm{E}+07$ & $4,72 \mathrm{E}+07$ & $6,07 \mathrm{E}+07$ & $5,30 \mathrm{E}+07$ \\
\hline $5,95 \mathrm{E}+07$ & $5,58 \mathrm{E}+07$ & $5,66 \mathrm{E}+07$ & $4,70 \mathrm{E}+07$ & $6,05 \mathrm{E}+07$ & $5,35 \mathrm{E}+07$ \\
\hline $5,93 \mathrm{E}+07$ & $5,60 \mathrm{E}+07$ & $5,66 \mathrm{E}+07$ & $4,70 \mathrm{E}+07$ & $6,04 \mathrm{E}+07$ & $5,38 \mathrm{E}+07$ \\
\hline & $5,53 \mathrm{E}+07$ & $5,66 \mathrm{E}+07$ & $4,70 \mathrm{E}+07$ & $6,03 \mathrm{E}+07$ & $5,41 \mathrm{E}+07$ \\
\hline & & $5,68 \mathrm{E}+07$ & & & $5,45 \mathrm{E}+07$ \\
\hline & & & & & $5,50 \mathrm{E}+07$ \\
\hline & & & & & $5,54 \mathrm{E}+07$ \\
\hline & & & & & \\
\hline
\end{tabular}

Continua 
Continuação do Anexo D

\begin{tabular}{|c|c|c|c|c|c|}
\hline 19 & 20 & 21 & 22 & 23 & 24 \\
\hline $1,61 \mathrm{E}+06$ & $1,56 \mathrm{E}+06$ & $1,48 \mathrm{E}+06$ & $1,58 \mathrm{E}+06$ & $1,48 \mathrm{E}+06$ & $1,45 \mathrm{E}+06$ \\
\hline $7,00 \mathrm{E}+06$ & $4,24 \mathrm{E}+06$ & $6,69 \mathrm{E}+06$ & $4,55 \mathrm{E}+06$ & $4,32 \mathrm{E}+06$ & $4,30 \mathrm{E}+06$ \\
\hline $1,29 \mathrm{E}+07$ & $8,74 \mathrm{E}+06$ & $1,12 \mathrm{E}+07$ & $9,11 \mathrm{E}+06$ & $8,24 \mathrm{E}+06$ & $7,51 \mathrm{E}+06$ \\
\hline $2,03 \mathrm{E}+07$ & $1,59 \mathrm{E}+07$ & $1,80 \mathrm{E}+07$ & $1,40 \mathrm{E}+07$ & $1,31 \mathrm{E}+07$ & $1,13 \mathrm{E}+07$ \\
\hline $2,72 \mathrm{E}+07$ & $2,28 \mathrm{E}+07$ & $2,45 \mathrm{E}+07$ & $1,98 \mathrm{E}+07$ & $1,84 \mathrm{E}+07$ & $1,58 \mathrm{E}+07$ \\
\hline $3,35 \mathrm{E}+07$ & $2,94 \mathrm{E}+07$ & $3,09 E+07$ & $2,64 \mathrm{E}+07$ & $2,36 \mathrm{E}+07$ & $2,02 \mathrm{E}+07$ \\
\hline $3,89 \mathrm{E}+07$ & $3,57 \mathrm{E}+07$ & $3,61 E+07$ & $3,22 \mathrm{E}+07$ & $2,91 \mathrm{E}+07$ & $2,45 \mathrm{E}+07$ \\
\hline $4,38 \mathrm{E}+07$ & $4,11 \mathrm{E}+07$ & $4,04 \mathrm{E}+07$ & $3,71 \mathrm{E}+07$ & $3,42 E+07$ & $2,94 \mathrm{E}+07$ \\
\hline $4,77 \mathrm{E}+07$ & $4,82 \mathrm{E}+07$ & $4,45 \mathrm{E}+07$ & $4,18 \mathrm{E}+07$ & $3,87 \mathrm{E}+07$ & $3,42 \mathrm{E}+07$ \\
\hline $5,07 \mathrm{E}+07$ & $5,06 \mathrm{E}+07$ & $4,75 \mathrm{E}+07$ & $4,56 \mathrm{E}+07$ & $4,33 \mathrm{E}+07$ & $3,92 \mathrm{E}+07$ \\
\hline $5,34 \mathrm{E}+07$ & $5,28 \mathrm{E}+07$ & $4,95 \mathrm{E}+07$ & $4,93 \mathrm{E}+07$ & $4,80 E+07$ & $4,33 \mathrm{E}+07$ \\
\hline $5,51 \mathrm{E}+07$ & $5,46 \mathrm{E}+07$ & $5,05 E+07$ & $5,20 \mathrm{E}+07$ & $5,19 \mathrm{E}+07$ & $4,71 \mathrm{E}+07$ \\
\hline $5,61 \mathrm{E}+07$ & $5,53 \mathrm{E}+07$ & $5,10 \mathrm{E}+07$ & $5,38 \mathrm{E}+07$ & $5,43 \mathrm{E}+07$ & $4,97 \mathrm{E}+07$ \\
\hline $5,65 \mathrm{E}+07$ & $5,57 \mathrm{E}+07$ & $5,12 \mathrm{E}+07$ & $5,52 \mathrm{E}+07$ & $5,64 \mathrm{E}+07$ & $5,21 \mathrm{E}+07$ \\
\hline $5,67 \mathrm{E}+07$ & $5,58 \mathrm{E}+07$ & $5,13 \mathrm{E}+07$ & $5,58 \mathrm{E}+07$ & $5,75 \mathrm{E}+07$ & $5,41 \mathrm{E}+07$ \\
\hline $5,66 \mathrm{E}+07$ & $5,58 \mathrm{E}+07$ & $5,13 \mathrm{E}+07$ & $5,62 \mathrm{E}+07$ & $5,83 \mathrm{E}+07$ & $5,55 \mathrm{E}+07$ \\
\hline $5,65 \mathrm{E}+07$ & $5,57 \mathrm{E}+07$ & $5,14 \mathrm{E}+07$ & $5,64 \mathrm{E}+07$ & $5,86 \mathrm{E}+07$ & $5,65 \mathrm{E}+07$ \\
\hline $5,65 \mathrm{E}+07$ & $5,57 \mathrm{E}+07$ & $5,14 \mathrm{E}+07$ & $5,66 \mathrm{E}+07$ & $5,87 \mathrm{E}+07$ & $5,70 \mathrm{E}+07$ \\
\hline $5,65 \mathrm{E}+07$ & $5,58 \mathrm{E}+07$ & $5,15 \mathrm{E}+07$ & $5,67 \mathrm{E}+07$ & $5,86 \mathrm{E}+07$ & $5,73 \mathrm{E}+07$ \\
\hline $5,64 \mathrm{E}+07$ & $5,60 \mathrm{E}+07$ & $5,15 \mathrm{E}+07$ & $5,67 \mathrm{E}+07$ & $5,85 \mathrm{E}+07$ & $5,75 \mathrm{E}+07$ \\
\hline \multirow[t]{6}{*}{$5,64 \mathrm{E}+07$} & $5,62 \mathrm{E}+07$ & $5,16 \mathrm{E}+07$ & $5,69 \mathrm{E}+07$ & $5,84 \mathrm{E}+07$ & $5,74 \mathrm{E}+07$ \\
\hline & & & $5,70 \mathrm{E}+07$ & & $5,74 \mathrm{E}+07$ \\
\hline & & & & & $5,74 \mathrm{E}+07$ \\
\hline & & & & & $5,74 \mathrm{E}+07$ \\
\hline & & & & & \\
\hline & & & & & \\
\hline
\end{tabular}

Continua 
Continuação do Anexo D

\begin{tabular}{|c|c|c|c|c|c|}
\hline 25 & 26 & 27 & 28 & 29 & 30 \\
\hline $1,49 \mathrm{E}+06$ & $1,60 \mathrm{E}+06$ & $1,62 \mathrm{E}+06$ & $1,30 \mathrm{E}+06$ & $1,46 \mathrm{E}+06$ & $1,47 \mathrm{E}+06$ \\
\hline $4,54 \mathrm{E}+06$ & $3,89 \mathrm{E}+06$ & $4,96 \mathrm{E}+06$ & $3,50 \mathrm{E}+06$ & $4,93 \mathrm{E}+06$ & $4,62 \mathrm{E}+06$ \\
\hline $7,32 \mathrm{E}+06$ & $6,93 \mathrm{E}+06$ & $8,35 \mathrm{E}+06$ & $5,58 \mathrm{E}+06$ & $9,47 \mathrm{E}+06$ & $7,90 \mathrm{E}+06$ \\
\hline $1,11 \mathrm{E}+07$ & $1,07 \mathrm{E}+07$ & $1,30 \mathrm{E}+07$ & $9,18 \mathrm{E}+06$ & $1,53 \mathrm{E}+07$ & $1,29 \mathrm{E}+07$ \\
\hline $1,54 \mathrm{E}+07$ & $1,53 \mathrm{E}+07$ & $1,83 \mathrm{E}+07$ & $1,37 \mathrm{E}+07$ & $2,20 \mathrm{E}+07$ & $1,74 \mathrm{E}+07$ \\
\hline $1,90 \mathrm{E}+07$ & $1,98 \mathrm{E}+07$ & $2,41 \mathrm{E}+07$ & $1,84 \mathrm{E}+07$ & $2,83 \mathrm{E}+07$ & $2,23 \mathrm{E}+07$ \\
\hline $2,23 \mathrm{E}+07$ & $2,43 \mathrm{E}+07$ & $2,93 \mathrm{E}+07$ & $2,33 \mathrm{E}+07$ & $3,42 \mathrm{E}+07$ & $2,68 \mathrm{E}+07$ \\
\hline $2,67 \mathrm{E}+07$ & $2,87 \mathrm{E}+07$ & $3,44 \mathrm{E}+07$ & $2,85 \mathrm{E}+07$ & $3,94 \mathrm{E}+07$ & $3,15 \mathrm{E}+07$ \\
\hline $3,02 \mathrm{E}+07$ & $3,28 \mathrm{E}+07$ & $3,98 \mathrm{E}+07$ & $3,36 \mathrm{E}+07$ & $4,39 \mathrm{E}+07$ & $3,58 \mathrm{E}+07$ \\
\hline $3,37 \mathrm{E}+07$ & $3,66 \mathrm{E}+07$ & $4,57 \mathrm{E}+07$ & $3,88 \mathrm{E}+07$ & $4,85 \mathrm{E}+07$ & $4,02 \mathrm{E}+07$ \\
\hline $3,71 \mathrm{E}+07$ & $4,06 \mathrm{E}+07$ & $4,93 \mathrm{E}+07$ & $4,30 \mathrm{E}+07$ & $5,16 \mathrm{E}+07$ & $4,42 \mathrm{E}+07$ \\
\hline $4,04 \mathrm{E}+07$ & $4,40 \mathrm{E}+07$ & $5,30 \mathrm{E}+07$ & $4,71 \mathrm{E}+07$ & $5,41 \mathrm{E}+07$ & $4,96 \mathrm{E}+07$ \\
\hline $4,24 \mathrm{E}+07$ & $4,73 \mathrm{E}+07$ & $5,61 \mathrm{E}+07$ & $5,01 \mathrm{E}+07$ & $5,60 \mathrm{E}+07$ & $5,28 \mathrm{E}+07$ \\
\hline $4,43 \mathrm{E}+07$ & $5,08 \mathrm{E}+07$ & $5,83 \mathrm{E}+07$ & $5,25 \mathrm{E}+07$ & $5,69 \mathrm{E}+07$ & $5,55 \mathrm{E}+07$ \\
\hline $4,56 \mathrm{E}+07$ & $5,32 \mathrm{E}+07$ & $5,99 \mathrm{E}+07$ & $5,42 \mathrm{E}+07$ & $5,74 \mathrm{E}+07$ & $5,74 \mathrm{E}+07$ \\
\hline $4,67 \mathrm{E}+07$ & $5,52 \mathrm{E}+07$ & $6,12 \mathrm{E}+07$ & $5,54 \mathrm{E}+07$ & $5,76 \mathrm{E}+07$ & $5,89 \mathrm{E}+07$ \\
\hline $4,77 \mathrm{E}+07$ & $5,65 \mathrm{E}+07$ & $6,19 \mathrm{E}+07$ & $5,60 \mathrm{E}+07$ & $5,76 \mathrm{E}+07$ & $5,98 \mathrm{E}+07$ \\
\hline $4,83 \mathrm{E}+07$ & $5,76 \mathrm{E}+07$ & $6,24 \mathrm{E}+07$ & $5,62 \mathrm{E}+07$ & $5,76 \mathrm{E}+07$ & $6,02 \mathrm{E}+07$ \\
\hline $4,89 \mathrm{E}+07$ & $5,83 \mathrm{E}+07$ & $6,26 \mathrm{E}+07$ & $5,62 \mathrm{E}+07$ & $5,72 \mathrm{E}+07$ & $6,03 \mathrm{E}+07$ \\
\hline $4,93 \mathrm{E}+07$ & $5,87 \mathrm{E}+07$ & $6,27 \mathrm{E}+07$ & $5,62 \mathrm{E}+07$ & $5,71 \mathrm{E}+07$ & $6,02 \mathrm{E}+07$ \\
\hline $4,97 \mathrm{E}+07$ & $5,89 \mathrm{E}+07$ & $6,30 \mathrm{E}+07$ & $5,60 \mathrm{E}+07$ & $5,69 \mathrm{E}+07$ & $6,01 \mathrm{E}+07$ \\
\hline $5,00 \mathrm{E}+07$ & $5,90 \mathrm{E}+07$ & $6,22 \mathrm{E}+07$ & & & $6,00 \mathrm{E}+07$ \\
\hline $5,02 \mathrm{E}+07$ & $5,90 \mathrm{E}+07$ & $6,24 \mathrm{E}+07$ & & & $5,99 \mathrm{E}+07$ \\
\hline $5,04 \mathrm{E}+07$ & $5,92 \mathrm{E}+07$ & & & & \\
\hline $5,05 \mathrm{E}+07$ & & & & & \\
\hline & & & & & \\
\hline
\end{tabular}

Continua 
Continuação do Anexo D

\begin{tabular}{|c|c|c|c|c|c|}
\hline 31 & 32 & 33 & 34 & 35 & 36 \\
\hline $1,53 \mathrm{E}+06$ & $1,44 \mathrm{E}+06$ & $1,51 \mathrm{E}+06$ & $1,72 \mathrm{E}+06$ & $1,55 \mathrm{E}+06$ & $1,52 \mathrm{E}+06$ \\
\hline $4,80 \mathrm{E}+06$ & $2,95 \mathrm{E}+06$ & $6,22 \mathrm{E}+06$ & $5,41 \mathrm{E}+06$ & $4,47 \mathrm{E}+06$ & $4,78 \mathrm{E}+06$ \\
\hline $8,72 \mathrm{E}+06$ & $5,20 \mathrm{E}+06$ & $1,25 \mathrm{E}+07$ & $1,09 \mathrm{E}+07$ & $8,71 \mathrm{E}+06$ & $7,75 \mathrm{E}+06$ \\
\hline $1,47 \mathrm{E}+07$ & $8,76 \mathrm{E}+06$ & $1,93 \mathrm{E}+07$ & $1,75 \mathrm{E}+07$ & $1,40 \mathrm{E}+07$ & $1,22 \mathrm{E}+07$ \\
\hline $2,08 \mathrm{E}+07$ & $1,33 \mathrm{E}+07$ & $2,58 \mathrm{E}+07$ & $2,38 \mathrm{E}+07$ & $1,91 \mathrm{E}+07$ & $1,85 \mathrm{E}+07$ \\
\hline $2,63 \mathrm{E}+07$ & $1,85 \mathrm{E}+07$ & $3,21 \mathrm{E}+07$ & $2,95 \mathrm{E}+07$ & $2,54 \mathrm{E}+07$ & $2,42 \mathrm{E}+07$ \\
\hline $3,17 \mathrm{E}+07$ & $2,39 \mathrm{E}+07$ & $3,74 \mathrm{E}+07$ & $3,46 \mathrm{E}+07$ & $3,41 \mathrm{E}+07$ & $3,00 \mathrm{E}+07$ \\
\hline $3,68 \mathrm{E}+07$ & $2,88 \mathrm{E}+07$ & $4,20 \mathrm{E}+07$ & $3,84 \mathrm{E}+07$ & $3,83 \mathrm{E}+07$ & $3,52 \mathrm{E}+07$ \\
\hline $4,09 \mathrm{E}+07$ & $3,34 \mathrm{E}+07$ & $4,57 \mathrm{E}+07$ & $4,21 \mathrm{E}+07$ & $4,22 \mathrm{E}+07$ & $4,02 \mathrm{E}+07$ \\
\hline $4,45 \mathrm{E}+07$ & $3,77 \mathrm{E}+07$ & $4,91 \mathrm{E}+07$ & $4,49 \mathrm{E}+07$ & $4,51 \mathrm{E}+07$ & $4,50 \mathrm{E}+07$ \\
\hline $4,75 \mathrm{E}+07$ & $4,13 \mathrm{E}+07$ & $5,13 \mathrm{E}+07$ & $4,65 \mathrm{E}+07$ & $4,71 \mathrm{E}+07$ & $4,92 \mathrm{E}+07$ \\
\hline $4,98 \mathrm{E}+07$ & $4,58 \mathrm{E}+07$ & $5,27 \mathrm{E}+07$ & $4,77 \mathrm{E}+07$ & $4,87 \mathrm{E}+07$ & $5,29 \mathrm{E}+07$ \\
\hline $5,12 \mathrm{E}+07$ & $4,75 \mathrm{E}+07$ & $5,34 \mathrm{E}+07$ & $4,83 \mathrm{E}+07$ & $4,98 \mathrm{E}+07$ & $5,54 \mathrm{E}+07$ \\
\hline $5,20 \mathrm{E}+07$ & $5,00 \mathrm{E}+07$ & $5,35 \mathrm{E}+07$ & $4,88 \mathrm{E}+07$ & $5,07 \mathrm{E}+07$ & $5,72 \mathrm{E}+07$ \\
\hline $5,26 \mathrm{E}+07$ & $5,20 \mathrm{E}+07$ & $5,35 \mathrm{E}+07$ & $4,91 \mathrm{E}+07$ & $5,15 \mathrm{E}+07$ & $5,83 \mathrm{E}+07$ \\
\hline $5,32 \mathrm{E}+07$ & $5,33 \mathrm{E}+07$ & $5,33 \mathrm{E}+07$ & $4,94 \mathrm{E}+07$ & $5,20 \mathrm{E}+07$ & $5,89 \mathrm{E}+07$ \\
\hline $5,37 \mathrm{E}+07$ & $5,42 \mathrm{E}+07$ & $5,31 \mathrm{E}+07$ & $4,96 \mathrm{E}+07$ & $5,24 \mathrm{E}+07$ & $5,91 \mathrm{E}+07$ \\
\hline $5,42 \mathrm{E}+07$ & $5,54 \mathrm{E}+07$ & $5,30 \mathrm{E}+07$ & $4,97 \mathrm{E}+07$ & $5,26 \mathrm{E}+07$ & $5,93 \mathrm{E}+07$ \\
\hline $5,46 \mathrm{E}+07$ & $5,62 \mathrm{E}+07$ & $5,30 \mathrm{E}+07$ & $5,00 \mathrm{E}+07$ & $5,29 \mathrm{E}+07$ & $5,92 \mathrm{E}+07$ \\
\hline $5,49 \mathrm{E}+07$ & $5,69 \mathrm{E}+07$ & $5,30 \mathrm{E}+07$ & $5,01 \mathrm{E}+07$ & $5,31 \mathrm{E}+07$ & $5,91 \mathrm{E}+07$ \\
\hline $5,53 \mathrm{E}+07$ & $5,75 \mathrm{E}+07$ & $5,30 \mathrm{E}+07$ & $5,04 \mathrm{E}+07$ & $5,33 \mathrm{E}+07$ & $5,90 \mathrm{E}+07$ \\
\hline $5,57 \mathrm{E}+07$ & $5,80 \mathrm{E}+07$ & & $5,07 \mathrm{E}+07$ & $5,35 \mathrm{E}+07$ & \\
\hline $5,62 \mathrm{E}+07$ & $5,87 \mathrm{E}+07$ & & & $5,37 \mathrm{E}+07$ & \\
\hline $5,65 \mathrm{E}+07$ & $5,94 \mathrm{E}+07$ & & & & \\
\hline & $5,99 \mathrm{E}+07$ & & & & \\
\hline & $6,04 \mathrm{E}+07$ & & & & \\
\hline
\end{tabular}

Continua 
Continuação do Anexo D

\begin{tabular}{|c|c|c|c|c|c|}
\hline 37 & 38 & 39 & 40 & 41 & 42 \\
\hline $1,65 \mathrm{E}+06$ & $1,67 \mathrm{E}+06$ & $1,55 \mathrm{E}+06$ & $1,63 \mathrm{E}+06$ & $1,36 \mathrm{E}+06$ & $1,54 \mathrm{E}+06$ \\
\hline $4,84 \mathrm{E}+06$ & $4,23 \mathrm{E}+06$ & $4,91 \mathrm{E}+06$ & $5,69 \mathrm{E}+06$ & $5,21 \mathrm{E}+06$ & $4,44 \mathrm{E}+06$ \\
\hline $8,84 \mathrm{E}+06$ & $7,59 \mathrm{E}+06$ & $8,83 \mathrm{E}+06$ & $1,10 \mathrm{E}+07$ & $9,63 \mathrm{E}+06$ & $8,20 \mathrm{E}+06$ \\
\hline $1,30 \mathrm{E}+07$ & $1,23 \mathrm{E}+07$ & $1,49 \mathrm{E}+07$ & $1,84 \mathrm{E}+07$ & $1,49 \mathrm{E}+07$ & $1,35 \mathrm{E}+07$ \\
\hline $1,84 \mathrm{E}+07$ & $1,74 \mathrm{E}+07$ & $2,17 \mathrm{E}+07$ & $2,56 \mathrm{E}+07$ & $2,00 \mathrm{E}+07$ & $1,95 \mathrm{E}+07$ \\
\hline $2,50 \mathrm{E}+07$ & $2,35 \mathrm{E}+07$ & $2,81 \mathrm{E}+07$ & $3,25 \mathrm{E}+07$ & $2,57 \mathrm{E}+07$ & $2,50 \mathrm{E}+07$ \\
\hline $3,13 \mathrm{E}+07$ & $2,90 \mathrm{E}+07$ & $3,39 \mathrm{E}+07$ & $3,82 \mathrm{E}+07$ & $3,16 \mathrm{E}+07$ & $3,10 \mathrm{E}+07$ \\
\hline $3,71 \mathrm{E}+07$ & $3,38 \mathrm{E}+07$ & $3,93 \mathrm{E}+07$ & $4,31 \mathrm{E}+07$ & $3,66 \mathrm{E}+07$ & $3,59 \mathrm{E}+07$ \\
\hline $4,22 \mathrm{E}+07$ & $3,92 \mathrm{E}+07$ & $4,44 \mathrm{E}+07$ & $4,73 \mathrm{E}+07$ & $4,13 \mathrm{E}+07$ & $3,94 \mathrm{E}+07$ \\
\hline $4,76 \mathrm{E}+07$ & $4,41 \mathrm{E}+07$ & $4,87 \mathrm{E}+07$ & $5,04 \mathrm{E}+07$ & $4,57 \mathrm{E}+07$ & $4,34 \mathrm{E}+07$ \\
\hline $5,16 \mathrm{E}+07$ & $4,80 \mathrm{E}+07$ & $5,20 \mathrm{E}+07$ & $5,31 \mathrm{E}+07$ & $5,00 \mathrm{E}+07$ & $4,63 \mathrm{E}+07$ \\
\hline $5,44 \mathrm{E}+07$ & $5,09 \mathrm{E}+07$ & $5,40 \mathrm{E}+07$ & $5,45 \mathrm{E}+07$ & $5,37 \mathrm{E}+07$ & $4,86 \mathrm{E}+07$ \\
\hline $5,65 \mathrm{E}+07$ & $5,31 \mathrm{E}+07$ & $5,54 \mathrm{E}+07$ & $5,51 \mathrm{E}+07$ & $5,58 \mathrm{E}+07$ & $5,00 \mathrm{E}+07$ \\
\hline $5,79 \mathrm{E}+07$ & $5,50 \mathrm{E}+07$ & $5,62 \mathrm{E}+07$ & $5,54 \mathrm{E}+07$ & $5,75 \mathrm{E}+07$ & $5,10 \mathrm{E}+07$ \\
\hline $5,85 \mathrm{E}+07$ & $5,63 \mathrm{E}+07$ & $5,67 \mathrm{E}+07$ & $5,53 \mathrm{E}+07$ & $5,85 \mathrm{E}+07$ & $5,15 \mathrm{E}+07$ \\
\hline $5,89 \mathrm{E}+07$ & $5,72 \mathrm{E}+07$ & $5,70 \mathrm{E}+07$ & $5,52 \mathrm{E}+07$ & $5,90 \mathrm{E}+07$ & $5,19 \mathrm{E}+07$ \\
\hline $5,90 \mathrm{E}+07$ & $5,79 \mathrm{E}+07$ & $5,73 \mathrm{E}+07$ & $5,55 \mathrm{E}+07$ & $5,93 \mathrm{E}+07$ & $5,21 \mathrm{E}+07$ \\
\hline $5,89 \mathrm{E}+07$ & $5,85 \mathrm{E}+07$ & $5,74 \mathrm{E}+07$ & $5,58 \mathrm{E}+07$ & $5,94 \mathrm{E}+07$ & $5,22 \mathrm{E}+07$ \\
\hline $5,89 \mathrm{E}+07$ & $5,89 \mathrm{E}+07$ & $5,76 \mathrm{E}+07$ & $5,62 \mathrm{E}+07$ & $5,94 \mathrm{E}+07$ & $5,23 \mathrm{E}+07$ \\
\hline $5,89 \mathrm{E}+07$ & $5,91 \mathrm{E}+07$ & $5,79 \mathrm{E}+07$ & $5,66 \mathrm{E}+07$ & $5,94 \mathrm{E}+07$ & $5,23 \mathrm{E}+07$ \\
\hline $5,90 \mathrm{E}+07$ & $5,94 \mathrm{E}+07$ & $5,83 \mathrm{E}+07$ & $5,70 \mathrm{E}+07$ & $5,94 \mathrm{E}+07$ & $5,24 \mathrm{E}+07$ \\
\hline $5,91 \mathrm{E}+07$ & $5,97 \mathrm{E}+07$ & $5,87 \mathrm{E}+07$ & $5,73 \mathrm{E}+07$ & $5,95 \mathrm{E}+07$ & \\
\hline $5,95 \mathrm{E}+07$ & $5,99 \mathrm{E}+07$ & $5,93 \mathrm{E}+07$ & $5,77 \mathrm{E}+07$ & & \\
\hline $5,96 \mathrm{E}+07$ & $6,01 \mathrm{E}+07$ & $5,97 \mathrm{E}+07$ & & & \\
\hline & $6,02 \mathrm{E}+07$ & $6,00 \mathrm{E}+07$ & & & \\
\hline & $6,05 \mathrm{E}+07$ & $6,05 \mathrm{E}+07$ & & & \\
\hline
\end{tabular}

Continua 
Continuação do Anexo D

\begin{tabular}{|c|c|c|c|c|r|}
\hline 43 & 44 & 45 & 46 & 47 & Mediana \\
\hline $1,63 \mathrm{E}+06$ & $1,52 \mathrm{E}+06$ & $1,60 \mathrm{E}+06$ & $1,55 \mathrm{E}+06$ & $1,45 \mathrm{E}+06$ & 1536,12 \\
\hline $4,74 \mathrm{E}+06$ & $3,66 \mathrm{E}+06$ & $3,86 \mathrm{E}+06$ & $3,63 \mathrm{E}+06$ & $3,33 \mathrm{E}+06$ & 4545,35 \\
\hline $8,72 \mathrm{E}+06$ & $7,65 \mathrm{E}+06$ & $8,35 \mathrm{E}+06$ & $6,72 \mathrm{E}+06$ & $5,69 \mathrm{E}+06$ & 8708,13 \\
\hline $1,50 \mathrm{E}+07$ & $1,28 \mathrm{E}+07$ & $1,48 \mathrm{E}+07$ & $1,12 \mathrm{E}+07$ & $8,86 \mathrm{E}+06$ & 14001,74 \\
\hline $2,13 \mathrm{E}+07$ & $1,91 \mathrm{E}+07$ & $2,17 \mathrm{E}+07$ & $1,54 \mathrm{E}+07$ & $1,28 \mathrm{E}+07$ & 19539,43 \\
\hline $2,78 \mathrm{E}+07$ & $2,35 \mathrm{E}+07$ & $2,72 \mathrm{E}+07$ & $2,08 \mathrm{E}+07$ & $1,73 \mathrm{E}+07$ & 25367,47 \\
\hline $3,36 \mathrm{E}+07$ & $2,73 \mathrm{E}+07$ & $3,29 \mathrm{E}+07$ & $2,57 \mathrm{E}+07$ & $2,20 \mathrm{E}+07$ & 31148,45 \\
\hline $3,90 \mathrm{E}+07$ & $3,11 \mathrm{E}+07$ & $3,82 \mathrm{E}+07$ & $3,07 \mathrm{E}+07$ & $2,71 \mathrm{E}+07$ & 36706,02 \\
\hline $4,43 \mathrm{E}+07$ & $3,50 \mathrm{E}+07$ & $4,31 \mathrm{E}+07$ & $3,57 \mathrm{E}+07$ & $3,18 \mathrm{E}+07$ & 41152,18 \\
\hline $4,89 \mathrm{E}+07$ & $3,83 \mathrm{E}+07$ & $4,78 \mathrm{E}+07$ & $4,16 \mathrm{E}+07$ & $3,73 \mathrm{E}+07$ & 44970,51 \\
\hline $5,26 \mathrm{E}+07$ & $4,08 \mathrm{E}+07$ & $5,12 \mathrm{E}+07$ & $4,48 \mathrm{E}+07$ & $4,15 \mathrm{E}+07$ & 48239,72 \\
\hline $5,48 \mathrm{E}+07$ & $4,22 \mathrm{E}+07$ & $5,37 \mathrm{E}+07$ & $4,87 \mathrm{E}+07$ & $4,55 \mathrm{E}+07$ & 51264,41 \\
\hline $5,63 \mathrm{E}+07$ & $4,47 \mathrm{E}+07$ & $5,62 \mathrm{E}+07$ & $5,15 \mathrm{E}+07$ & $4,84 \mathrm{E}+07$ & 53106,62 \\
\hline $5,72 \mathrm{E}+07$ & $4,61 \mathrm{E}+07$ & $5,72 \mathrm{E}+07$ & $5,41 \mathrm{E}+07$ & $5,10 \mathrm{E}+07$ & 54243,72 \\
\hline $5,77 \mathrm{E}+07$ & $4,73 \mathrm{E}+07$ & $5,80 \mathrm{E}+07$ & $5,59 \mathrm{E}+07$ & $5,32 \mathrm{E}+07$ & 55121,14 \\
\hline $5,79 \mathrm{E}+07$ & $4,82 \mathrm{E}+07$ & $5,86 \mathrm{E}+07$ & $5,73 \mathrm{E}+07$ & $5,48 \mathrm{E}+07$ & 55641,82 \\
\hline $5,78 \mathrm{E}+07$ & $4,89 \mathrm{E}+07$ & $5,90 \mathrm{E}+07$ & $5,84 \mathrm{E}+07$ & $5,59 \mathrm{E}+07$ & 56274,99 \\
\hline $5,79 \mathrm{E}+07$ & $4,95 \mathrm{E}+07$ & $5,91 \mathrm{E}+07$ & $5,91 \mathrm{E}+07$ & $5,66 \mathrm{E}+07$ & 56528,30 \\
\hline $5,77 \mathrm{E}+07$ & $5,01 \mathrm{E}+07$ & $5,92 \mathrm{E}+07$ & $5,96 \mathrm{E}+07$ & $5,72 \mathrm{E}+07$ & 56502,26 \\
\hline $5,77 \mathrm{E}+07$ & $5,05 \mathrm{E}+07$ & $5,93 \mathrm{E}+07$ & $5,98 \mathrm{E}+07$ & $5,75 \mathrm{E}+07$ & 56671,58 \\
\hline $5,76 \mathrm{E}+07$ & $5,09 \mathrm{E}+07$ & $5,94 \mathrm{E}+07$ & $5,99 \mathrm{E}+07$ & $5,76 \mathrm{E}+07$ & 56938,28 \\
\hline $5,76 \mathrm{E}+07$ & $5,14 \mathrm{E}+07$ & $5,94 \mathrm{E}+07$ & $6,01 \mathrm{E}+07$ & $5,78 \mathrm{E}+07$ & 57444,14 \\
\hline $5,76 \mathrm{E}+07$ & $5,22 \mathrm{E}+07$ & $5,96 \mathrm{E}+07$ & $6,01 \mathrm{E}+07$ & $5,78 \mathrm{E}+07$ & 57663,76 \\
\hline & & & $6,00 \mathrm{E}+07$ & $5,79 \mathrm{E}+07$ & 57919,25 \\
\hline & & & $6,03 \mathrm{E}+07$ & & 57653,55 \\
\hline & & & $6,04 \mathrm{E}+07$ & & 60401,57 \\
\hline
\end{tabular}


ANEXO E

DADOS DOS CORPOS DE PROVA DO GRUPO 1V 


\begin{tabular}{|r|c|c|c|c|c|c|}
\cline { 2 - 7 } & $\mathbf{1}$ & $\mathbf{2}$ & $\mathbf{3}$ & $\mathbf{4}$ & $\mathbf{5}$ & $\mathbf{6}$ \\
\hline 0,00 & $1,38 \mathrm{E}+06$ & $1,44 \mathrm{E}+06$ & $1,31 \mathrm{E}+06$ & $1,55 \mathrm{E}+06$ & $1,45 \mathrm{E}+06$ & $1,47 \mathrm{E}+06$ \\
\hline 0,05 & $5,83 \mathrm{E}+06$ & $5,12 \mathrm{E}+06$ & $7,55 \mathrm{E}+06$ & $5,25 \mathrm{E}+06$ & $5,89 \mathrm{E}+06$ & $4,70 \mathrm{E}+06$ \\
\hline 0,10 & $1,16 \mathrm{E}+07$ & $8,74 \mathrm{E}+06$ & $1,21 \mathrm{E}+07$ & $1,02 \mathrm{E}+07$ & $1,11 \mathrm{E}+07$ & $8,70 \mathrm{E}+06$ \\
\hline 0,15 & $1,86 \mathrm{E}+07$ & $1,30 \mathrm{E}+07$ & $1,65 \mathrm{E}+07$ & $1,57 \mathrm{E}+07$ & $1,73 \mathrm{E}+07$ & $1,37 \mathrm{E}+07$ \\
\hline 0,20 & $2,52 \mathrm{E}+07$ & $1,77 \mathrm{E}+07$ & $2,18 \mathrm{E}+07$ & $2,27 \mathrm{E}+07$ & $2,42 \mathrm{E}+07$ & $1,97 \mathrm{E}+07$ \\
\hline 0,25 & $3,14 \mathrm{E}+07$ & $2,34 \mathrm{E}+07$ & $2,74 \mathrm{E}+07$ & $2,67 \mathrm{E}+07$ & $2,97 \mathrm{E}+07$ & $2,52 \mathrm{E}+07$ \\
\hline 0,30 & $3,67 \mathrm{E}+07$ & $2,88 \mathrm{E}+07$ & $3,29 \mathrm{E}+07$ & $3,64 \mathrm{E}+07$ & $3,49 \mathrm{E}+07$ & $3,13 \mathrm{E}+07$ \\
\hline 0,35 & $4,08 \mathrm{E}+07$ & $3,38 \mathrm{E}+07$ & $3,79 \mathrm{E}+07$ & $4,05 \mathrm{E}+07$ & $3,89 \mathrm{E}+07$ & $3,64 \mathrm{E}+07$ \\
\hline 0,40 & $4,51 \mathrm{E}+07$ & $3,85 \mathrm{E}+07$ & $4,17 \mathrm{E}+07$ & $4,39 \mathrm{E}+07$ & $4,23 \mathrm{E}+07$ & $4,10 \mathrm{E}+07$ \\
\hline 0,45 & $4,80 \mathrm{E}+07$ & $4,25 \mathrm{E}+07$ & $4,51 \mathrm{E}+07$ & $4,75 \mathrm{E}+07$ & $4,56 \mathrm{E}+07$ & $4,48 \mathrm{E}+07$ \\
\hline 0,50 & $5,01 \mathrm{E}+07$ & $4,57 \mathrm{E}+07$ & $4,80 \mathrm{E}+07$ & $5,01 \mathrm{E}+07$ & $4,77 \mathrm{E}+07$ & $4,80 \mathrm{E}+07$ \\
\hline 0,55 & $5,21 \mathrm{E}+07$ & $4,82 \mathrm{E}+07$ & $5,01 \mathrm{E}+07$ & $5,24 \mathrm{E}+07$ & $4,93 \mathrm{E}+07$ & $5,07 \mathrm{E}+07$ \\
\hline 0,60 & $5,32 \mathrm{E}+07$ & $5,04 \mathrm{E}+07$ & $5,16 \mathrm{E}+07$ & $5,40 \mathrm{E}+07$ & $5,05 \mathrm{E}+07$ & $5,30 \mathrm{E}+07$ \\
\hline 0,65 & $5,38 \mathrm{E}+07$ & $5,18 \mathrm{E}+07$ & $5,27 \mathrm{E}+07$ & $5,52 \mathrm{E}+07$ & $5,12 \mathrm{E}+07$ & $5,44 \mathrm{E}+07$ \\
\hline 0,70 & $5,41 \mathrm{E}+07$ & $5,29 \mathrm{E}+07$ & $5,35 \mathrm{E}+07$ & $5,60 \mathrm{E}+07$ & $5,17 \mathrm{E}+07$ & $5,55 \mathrm{E}+07$ \\
\hline 0,75 & $5,42 \mathrm{E}+07$ & $5,37 \mathrm{E}+07$ & $5,39 \mathrm{E}+07$ & $5,64 \mathrm{E}+07$ & $5,18 \mathrm{E}+07$ & $5,64 \mathrm{E}+07$ \\
\hline 0,80 & $5,42 \mathrm{E}+07$ & $5,42 \mathrm{E}+07$ & $5,42 \mathrm{E}+07$ & $5,66 \mathrm{E}+07$ & $5,18 \mathrm{E}+07$ & $5,70 \mathrm{E}+07$ \\
\hline 0,85 & $5,43 \mathrm{E}+07$ & $5,44 \mathrm{E}+07$ & $5,42 \mathrm{E}+07$ & $5,66 \mathrm{E}+07$ & $5,18 \mathrm{E}+07$ & $5,74 \mathrm{E}+07$ \\
\hline 0,90 & $5,42 \mathrm{E}+07$ & $5,46 \mathrm{E}+07$ & $5,43 \mathrm{E}+07$ & $5,66 \mathrm{E}+07$ & $5,19 \mathrm{E}+07$ & $5,76 \mathrm{E}+07$ \\
\hline 0,95 & $5,41 \mathrm{E}+07$ & $5,46 \mathrm{E}+07$ & $5,43 \mathrm{E}+07$ & $5,65 \mathrm{E}+07$ & $5,19 \mathrm{E}+07$ & $5,77 \mathrm{E}+07$ \\
\hline 1,00 & $5,41 \mathrm{E}+07$ & $5,46 \mathrm{E}+07$ & $5,44 \mathrm{E}+07$ & $5,66 \mathrm{E}+07$ & $5,19 \mathrm{E}+07$ & $5,77 \mathrm{E}+07$ \\
\hline 1,05 & $5,42 \mathrm{E}+07$ & $5,46 \mathrm{E}+07$ & $5,45 \mathrm{E}+07$ & $5,67 \mathrm{E}+07$ & $5,19 \mathrm{E}+07$ & $5,78 \mathrm{E}+07$ \\
\hline 1,10 & $5,42 \mathrm{E}+07$ & $5,46 \mathrm{E}+07$ & & & $5,19 \mathrm{E}+07$ & $5,79 \mathrm{E}+07$ \\
\hline & & & & & \\
\hline
\end{tabular}

Continua 
Continuação do Anexo E

\begin{tabular}{|c|c|c|c|c|c|}
\hline 7 & 8 & 9 & 10 & 11 & 12 \\
\hline $1,62 E+06$ & $1,62 E+06$ & $1,48 E+06$ & $1,55 E+06$ & $1,53 E+06$ & $1,57 \mathrm{E}+06$ \\
\hline $5,15 \mathrm{E}+06$ & $4,43 E+06$ & $3,96 E+06$ & $4,86 \mathrm{E}+06$ & $4,42 E+06$ & $5,47 E+06$ \\
\hline $9,79 E+06$ & $8,50 E+06$ & $7,16 \mathrm{E}+06$ & $9,40 E+06$ & $9,04 E+06$ & $1,14 \mathrm{E}+07$ \\
\hline $1,56 \mathrm{E}+07$ & $1,40 \mathrm{E}+07$ & $1,17 \mathrm{E}+07$ & $1,48 E+07$ & $1,49 \mathrm{E}+07$ & $1,79 E+07$ \\
\hline $2,22 \mathrm{E}+07$ & $2,04 \mathrm{E}+07$ & $1,70 \mathrm{E}+07$ & $2,03 E+07$ & $2,16 E+07$ & $2,45 E+07$ \\
\hline $2,85 E+07$ & $2,62 E+07$ & $2,26 \mathrm{E}+07$ & $2,56 \mathrm{E}+07$ & $2,67 E+07$ & $3,18 E+07$ \\
\hline $3,39 E+07$ & $3,16 \mathrm{E}+07$ & $2,86 \mathrm{E}+07$ & $3,07 E+07$ & $3,21 E+07$ & $3,66 \mathrm{E}+07$ \\
\hline $3,82 \mathrm{E}+07$ & $3,62 E+07$ & $3,37 \mathrm{E}+07$ & $3,50 E+07$ & $3,68 E+07$ & $4,15 E+07$ \\
\hline $4,21 \mathrm{E}+07$ & $4,01 \mathrm{E}+07$ & $3,88 E+07$ & $3,85 E+07$ & $4,05 E+07$ & $4,54 \mathrm{E}+07$ \\
\hline $4,50 \mathrm{E}+07$ & $4,38 \mathrm{E}+07$ & $4,32 E+07$ & $4,15 E+07$ & $4,32 \mathrm{E}+07$ & $4,83 E+07$ \\
\hline $4,71 \mathrm{E}+07$ & $4,71 \mathrm{E}+07$ & $4,63 E+07$ & $4,39 E+07$ & $4,57 \mathrm{E}+07$ & $5,08 E+07$ \\
\hline $4,85 \mathrm{E}+07$ & $4,94 \mathrm{E}+07$ & $4,89 \mathrm{E}+07$ & $4,58 \mathrm{E}+07$ & $4,73 E+07$ & $5,25 \mathrm{E}+07$ \\
\hline $4,94 \mathrm{E}+07$ & $5,12 \mathrm{E}+07$ & $5,08 E+07$ & $4,72 E+07$ & $4,85 E+07$ & $5,33 E+07$ \\
\hline $4,98 \mathrm{E}+07$ & $5,25 E+07$ & $5,21 \mathrm{E}+07$ & $4,82 E+07$ & $4,93 \mathrm{E}+07$ & $5,36 \mathrm{E}+07$ \\
\hline $5,01 \mathrm{E}+07$ & $5,32 \mathrm{E}+07$ & $5,28 \mathrm{E}+07$ & $4,90 \mathrm{E}+07$ & $4,98 \mathrm{E}+07$ & $5,36 \mathrm{E}+07$ \\
\hline $5,01 \mathrm{E}+07$ & $5,37 \mathrm{E}+07$ & $5,32 E+07$ & $4,96 \mathrm{E}+07$ & $5,04 \mathrm{E}+07$ & $5,34 \mathrm{E}+07$ \\
\hline $5,01 \mathrm{E}+07$ & $5,40 \mathrm{E}+07$ & $5,34 \mathrm{E}+07$ & $5,01 E+07$ & $5,07 \mathrm{E}+07$ & $5,32 \mathrm{E}+07$ \\
\hline $5,01 \mathrm{E}+07$ & $5,42 E+07$ & $5,36 \mathrm{E}+07$ & $5,05 E+07$ & $5,08 E+07$ & $5,30 E+07$ \\
\hline $5,01 \mathrm{E}+07$ & $5,43 \mathrm{E}+07$ & $5,36 \mathrm{E}+07$ & $5,09 E+07$ & $5,08 \mathrm{E}+07$ & $5,26 \mathrm{E}+07$ \\
\hline $5,00 \mathrm{E}+07$ & $5,44 \mathrm{E}+07$ & $5,36 \mathrm{E}+07$ & $5,12 E+07$ & $5,09 \mathrm{E}+07$ & $5,25 \mathrm{E}+07$ \\
\hline $5,01 \mathrm{E}+07$ & $5,45 \mathrm{E}+07$ & $5,37 \mathrm{E}+07$ & $5,17 E+07$ & $5,11 \mathrm{E}+07$ & $5,25 \mathrm{E}+07$ \\
\hline $5,03 E+07$ & $5,47 \mathrm{E}+07$ & $5,37 \mathrm{E}+07$ & $5,23 E+07$ & $5,13 E+07$ & $5,26 \mathrm{E}+07$ \\
\hline $5,03 E+07$ & $5,49 E+07$ & $5,38 E+07$ & & & $5,27 E+07$ \\
\hline
\end{tabular}

Continua 
Continuação do Anexo E

\begin{tabular}{|c|c|c|c|c|c|}
\hline 13 & 14 & 15 & 16 & 17 & 18 \\
\hline $1,42 \mathrm{E}+06$ & $1,41 \mathrm{E}+06$ & $1,54 \mathrm{E}+06$ & $1,51 \mathrm{E}+06$ & $1,43 \mathrm{E}+06$ & $1,45 \mathrm{E}+06$ \\
\hline $4,83 \mathrm{E}+06$ & $4,01 \mathrm{E}+06$ & $5,67 \mathrm{E}+06$ & $4,10 \mathrm{E}+06$ & $4,95 \mathrm{E}+06$ & $5,12 \mathrm{E}+06$ \\
\hline $8,40 \mathrm{E}+06$ & $7,76 \mathrm{E}+06$ & $1,11 \mathrm{E}+07$ & $7,99 \mathrm{E}+06$ & $9,15 \mathrm{E}+06$ & $9,38 \mathrm{E}+06$ \\
\hline $1,36 \mathrm{E}+07$ & $1,30 \mathrm{E}+07$ & $2,43 \mathrm{E}+07$ & $1,37 \mathrm{E}+07$ & $1,43 \mathrm{E}+07$ & $1,48 \mathrm{E}+07$ \\
\hline $1,88 \mathrm{E}+07$ & $1,86 \mathrm{E}+07$ & $2,96 \mathrm{E}+07$ & $1,96 \mathrm{E}+07$ & $2,00 \mathrm{E}+07$ & $2,04 \mathrm{E}+07$ \\
\hline $2,40 \mathrm{E}+07$ & $2,46 \mathrm{E}+07$ & $3,42 \mathrm{E}+07$ & $2,60 \mathrm{E}+07$ & $2,59 \mathrm{E}+07$ & $2,56 \mathrm{E}+07$ \\
\hline $2,92 \mathrm{E}+07$ & $3,08 \mathrm{E}+07$ & $3,83 \mathrm{E}+07$ & $3,17 \mathrm{E}+07$ & $3,15 \mathrm{E}+07$ & $3,06 \mathrm{E}+07$ \\
\hline $3,37 \mathrm{E}+07$ & $3,60 \mathrm{E}+07$ & $4,15 \mathrm{E}+07$ & $3,69 \mathrm{E}+07$ & $3,67 \mathrm{E}+07$ & $3,51 \mathrm{E}+07$ \\
\hline $3,81 \mathrm{E}+07$ & $4,07 \mathrm{E}+07$ & $4,33 \mathrm{E}+07$ & $4,06 \mathrm{E}+07$ & $4,13 \mathrm{E}+07$ & $3,85 \mathrm{E}+07$ \\
\hline $4,22 \mathrm{E}+07$ & $4,45 \mathrm{E}+07$ & $4,43 \mathrm{E}+07$ & $4,33 \mathrm{E}+07$ & $4,45 \mathrm{E}+07$ & $4,16 \mathrm{E}+07$ \\
\hline $4,58 \mathrm{E}+07$ & $4,75 \mathrm{E}+07$ & $4,47 \mathrm{E}+07$ & $4,55 \mathrm{E}+07$ & $4,71 \mathrm{E}+07$ & $4,40 \mathrm{E}+07$ \\
\hline $4,84 \mathrm{E}+07$ & $4,96 \mathrm{E}+07$ & $4,48 \mathrm{E}+07$ & $4,69 \mathrm{E}+07$ & $4,94 \mathrm{E}+07$ & $4,61 \mathrm{E}+07$ \\
\hline $5,08 \mathrm{E}+07$ & $5,10 \mathrm{E}+07$ & $4,48 \mathrm{E}+07$ & $4,76 \mathrm{E}+07$ & $5,08 \mathrm{E}+07$ & $4,75 \mathrm{E}+07$ \\
\hline $5,25 \mathrm{E}+07$ & $5,19 \mathrm{E}+07$ & $4,49 \mathrm{E}+07$ & $4,80 \mathrm{E}+07$ & $5,18 \mathrm{E}+07$ & $4,84 \mathrm{E}+07$ \\
\hline $5,34 \mathrm{E}+07$ & $5,25 \mathrm{E}+07$ & $4,50 \mathrm{E}+07$ & $4,84 \mathrm{E}+07$ & $5,24 \mathrm{E}+07$ & $4,90 \mathrm{E}+07$ \\
\hline $5,41 \mathrm{E}+07$ & $5,27 \mathrm{E}+07$ & $4,52 \mathrm{E}+07$ & $4,89 \mathrm{E}+07$ & $5,27 \mathrm{E}+07$ & $4,95 \mathrm{E}+07$ \\
\hline $5,45 \mathrm{E}+07$ & $5,28 \mathrm{E}+07$ & $4,55 \mathrm{E}+07$ & $4,94 \mathrm{E}+07$ & $5,28 \mathrm{E}+07$ & $4,97 \mathrm{E}+07$ \\
\hline $5,47 \mathrm{E}+07$ & $5,29 \mathrm{E}+07$ & $4,59 \mathrm{E}+07$ & $4,99 \mathrm{E}+07$ & $5,28 \mathrm{E}+07$ & $4,99 \mathrm{E}+07$ \\
\hline $5,47 \mathrm{E}+07$ & $5,30 \mathrm{E}+07$ & $4,64 \mathrm{E}+07$ & $5,04 \mathrm{E}+07$ & $5,27 \mathrm{E}+07$ & $5,01 \mathrm{E}+07$ \\
\hline $5,47 \mathrm{E}+07$ & $5,30 \mathrm{E}+07$ & $4,68 \mathrm{E}+07$ & $5,08 \mathrm{E}+07$ & $5,26 \mathrm{E}+07$ & $5,03 \mathrm{E}+07$ \\
\hline $5,45 \mathrm{E}+07$ & $5,29 \mathrm{E}+07$ & & $5,11 \mathrm{E}+07$ & $5,25 \mathrm{E}+07$ & $5,04 \mathrm{E}+07$ \\
\hline $5,45 \mathrm{E}+07$ & $5,31 \mathrm{E}+07$ & & $5,14 \mathrm{E}+07$ & $5,25 \mathrm{E}+07$ & $5,06 \mathrm{E}+07$ \\
\hline $5,45 \mathrm{E}+07$ & $5,33 \mathrm{E}+07$ & & $5,17 \mathrm{E}+07$ & $5,25 \mathrm{E}+07$ & $5,08 \mathrm{E}+07$ \\
\hline & & & $\mathrm{n}+07 n$ & & \\
\hline
\end{tabular}

Continua 
Continuação do Anexo E

\begin{tabular}{|c|c|c|c|c|c|}
\hline 19 & 20 & 21 & 22 & 23 & 29 \\
\hline $1,48 \mathrm{E}+06$ & $1,59 \mathrm{E}+06$ & $1,45 \mathrm{E}+06$ & $1,46 \mathrm{E}+06$ & $1,33 \mathrm{E}+06$ & $1,47 \mathrm{E}+06$ \\
\hline $4,51 \mathrm{E}+06$ & $5,72 \mathrm{E}+06$ & $4,08 \mathrm{E}+06$ & $5,29 \mathrm{E}+06$ & $4,24 \mathrm{E}+06$ & $5,07 \mathrm{E}+06$ \\
\hline $8,61 \mathrm{E}+06$ & $9,75 \mathrm{E}+06$ & $7,22 \mathrm{E}+06$ & $1,03 \mathrm{E}+07$ & $8,10 \mathrm{E}+06$ & $9,96 \mathrm{E}+06$ \\
\hline $1,42 \mathrm{E}+07$ & $2,00 \mathrm{E}+07$ & $1,26 \mathrm{E}+07$ & $1,58 \mathrm{E}+07$ & $1,24 \mathrm{E}+07$ & $1,57 \mathrm{E}+07$ \\
\hline $1,97 \mathrm{E}+07$ & $2,55 \mathrm{E}+07$ & $1,84 \mathrm{E}+07$ & $2,14 \mathrm{E}+07$ & $1,72 \mathrm{E}+07$ & $2,13 \mathrm{E}+07$ \\
\hline $2,53 \mathrm{E}+07$ & $3,00 \mathrm{E}+07$ & $2,43 \mathrm{E}+07$ & $2,70 \mathrm{E}+07$ & $2,27 \mathrm{E}+07$ & $2,70 \mathrm{E}+07$ \\
\hline $3,04 \mathrm{E}+07$ & $3,35 \mathrm{E}+07$ & $2,96 \mathrm{E}+07$ & $3,15 \mathrm{E}+07$ & $2,79 \mathrm{E}+07$ & $3,21 \mathrm{E}+07$ \\
\hline $3,47 \mathrm{E}+07$ & $3,69 \mathrm{E}+07$ & $3,47 \mathrm{E}+07$ & $3,61 \mathrm{E}+07$ & $3,25 \mathrm{E}+07$ & $3,66 \mathrm{E}+07$ \\
\hline $3,85 \mathrm{E}+07$ & $3,97 \mathrm{E}+07$ & $3,90 \mathrm{E}+07$ & $3,98 \mathrm{E}+07$ & $3,67 \mathrm{E}+07$ & $4,03 \mathrm{E}+07$ \\
\hline $4,14 \mathrm{E}+07$ & $4,20 \mathrm{E}+07$ & $4,32 \mathrm{E}+07$ & $4,20 \mathrm{E}+07$ & $4,01 \mathrm{E}+07$ & $4,36 \mathrm{E}+07$ \\
\hline $4,36 \mathrm{E}+07$ & $4,33 \mathrm{E}+07$ & $4,62 \mathrm{E}+07$ & $4,40 \mathrm{E}+07$ & $4,26 \mathrm{E}+07$ & $4,60 \mathrm{E}+07$ \\
\hline $4,51 \mathrm{E}+07$ & $4,42 \mathrm{E}+07$ & $4,87 \mathrm{E}+07$ & $4,54 \mathrm{E}+07$ & $4,51 \mathrm{E}+07$ & $4,81 \mathrm{E}+07$ \\
\hline $4,60 \mathrm{E}+07$ & $4,48 \mathrm{E}+07$ & $5,04 \mathrm{E}+07$ & $4,63 \mathrm{E}+07$ & $4,69 \mathrm{E}+07$ & $4,94 \mathrm{E}+07$ \\
\hline $4,66 \mathrm{E}+07$ & $4,51 \mathrm{E}+07$ & $5,15 \mathrm{E}+07$ & $4,70 \mathrm{E}+07$ & $4,81 \mathrm{E}+07$ & $5,01 \mathrm{E}+07$ \\
\hline $4,68 \mathrm{E}+07$ & $4,54 \mathrm{E}+07$ & $4,41 \mathrm{E}+07$ & $4,75 \mathrm{E}+07$ & $4,91 \mathrm{E}+07$ & $5,05 \mathrm{E}+07$ \\
\hline $4,69 \mathrm{E}+07$ & $4,57 \mathrm{E}+07$ & $5,22 \mathrm{E}+07$ & $4,81 \mathrm{E}+07$ & $4,98 \mathrm{E}+07$ & $5,05 \mathrm{E}+07$ \\
\hline $4,69 \mathrm{E}+07$ & $4,61 \mathrm{E}+07$ & $5,23 \mathrm{E}+07$ & $4,86 \mathrm{E}+07$ & $5,03 \mathrm{E}+07$ & $5,05 \mathrm{E}+07$ \\
\hline $4,68 \mathrm{E}+07$ & $4,65 \mathrm{E}+07$ & $5,24 \mathrm{E}+07$ & $4,91 \mathrm{E}+07$ & $5,07 \mathrm{E}+07$ & $5,05 \mathrm{E}+07$ \\
\hline $4,67 \mathrm{E}+07$ & $4,69 \mathrm{E}+07$ & $4,45 \mathrm{E}+07$ & $4,95 \mathrm{E}+07$ & $5,10 \mathrm{E}+07$ & $5,05 \mathrm{E}+07$ \\
\hline $4,67 \mathrm{E}+07$ & $4,72 \mathrm{E}+07$ & $5,23 \mathrm{E}+07$ & $5,00 \mathrm{E}+07$ & $5,13 \mathrm{E}+07$ & $5,04 \mathrm{E}+07$ \\
\hline $4,67 \mathrm{E}+07$ & $4,76 \mathrm{E}+07$ & $5,23 \mathrm{E}+07$ & $5,03 \mathrm{E}+07$ & $5,16 \mathrm{E}+07$ & $5,05 \mathrm{E}+07$ \\
\hline $4,68 \mathrm{E}+07$ & $4,80 \mathrm{E}+07$ & $5,24 \mathrm{E}+07$ & $5,07 \mathrm{E}+07$ & $5,17 \mathrm{E}+07$ & $5,05 \mathrm{E}+07$ \\
\hline $4,68 \mathrm{E}+07$ & & $5,25 \mathrm{E}+07$ & $5,12 \mathrm{E}+07$ & $5,20 \mathrm{E}+07$ & $5,06 \mathrm{E}+07$ \\
\hline
\end{tabular}

Continua 
Continuação do Anexo E

\begin{tabular}{|c|c|c|c|c|c|}
\hline 25 & 26 & 27 & 28 & 42 & 30 \\
\hline $1,53 \mathrm{E}+06$ & $1,53 \mathrm{E}+06$ & $1,39 \mathrm{E}+06$ & $1,53 \mathrm{E}+06$ & $1,50 \mathrm{E}+06$ & $1,48 \mathrm{E}+06$ \\
\hline $5,50 \mathrm{E}+06$ & $4,55 \mathrm{E}+06$ & $4,96 \mathrm{E}+06$ & $4,97 \mathrm{E}+06$ & $4,94 \mathrm{E}+06$ & $6,06 \mathrm{E}+06$ \\
\hline $1,02 \mathrm{E}+07$ & $8,10 \mathrm{E}+06$ & $9,65 \mathrm{E}+06$ & $8,49 \mathrm{E}+06$ & $9,69 \mathrm{E}+06$ & $1,67 \mathrm{E}+07$ \\
\hline $1,60 \mathrm{E}+07$ & $1,26 \mathrm{E}+07$ & $1,54 \mathrm{E}+07$ & $1,33 \mathrm{E}+07$ & $1,57 \mathrm{E}+07$ & $1,96 \mathrm{E}+07$ \\
\hline $2,17 \mathrm{E}+07$ & $1,82 \mathrm{E}+07$ & $2,19 \mathrm{E}+07$ & $1,80 \mathrm{E}+07$ & $2,14 \mathrm{E}+07$ & $2,56 \mathrm{E}+07$ \\
\hline $2,76 \mathrm{E}+07$ & $2,43 \mathrm{E}+07$ & $2,78 \mathrm{E}+07$ & $2,30 \mathrm{E}+07$ & $2,75 \mathrm{E}+07$ & $3,17 \mathrm{E}+07$ \\
\hline $3,23 \mathrm{E}+07$ & $3,04 \mathrm{E}+07$ & $3,36 \mathrm{E}+07$ & $2,79 \mathrm{E}+07$ & $3,25 \mathrm{E}+07$ & $3,71 \mathrm{E}+07$ \\
\hline $3,66 \mathrm{E}+07$ & $3,56 \mathrm{E}+07$ & $3,90 \mathrm{E}+07$ & $3,26 \mathrm{E}+07$ & $3,75 \mathrm{E}+07$ & $4,19 \mathrm{E}+07$ \\
\hline $4,00 \mathrm{E}+07$ & $4,04 \mathrm{E}+07$ & $4,35 \mathrm{E}+07$ & $3,66 \mathrm{E}+07$ & $4,14 \mathrm{E}+07$ & $4,59 \mathrm{E}+07$ \\
\hline $4,25 \mathrm{E}+07$ & $4,45 \mathrm{E}+07$ & $4,70 \mathrm{E}+07$ & $4,01 \mathrm{E}+07$ & $4,45 \mathrm{E}+07$ & $4,83 \mathrm{E}+07$ \\
\hline $4,46 \mathrm{E}+07$ & $4,78 \mathrm{E}+07$ & $4,96 \mathrm{E}+07$ & $4,26 \mathrm{E}+07$ & $4,73 \mathrm{E}+07$ & $5,04 \mathrm{E}+07$ \\
\hline $4,63 \mathrm{E}+07$ & $5,05 \mathrm{E}+07$ & $5,10 \mathrm{E}+07$ & $4,50 \mathrm{E}+07$ & $4,92 \mathrm{E}+07$ & $5,17 \mathrm{E}+07$ \\
\hline $4,73 \mathrm{E}+07$ & $5,20 \mathrm{E}+07$ & $5,21 \mathrm{E}+07$ & $4,68 \mathrm{E}+07$ & $5,08 \mathrm{E}+07$ & $5,25 \mathrm{E}+07$ \\
\hline $4,83 \mathrm{E}+07$ & $5,29 \mathrm{E}+07$ & $5,27 \mathrm{E}+07$ & $4,83 \mathrm{E}+07$ & $5,17 \mathrm{E}+07$ & $5,28 \mathrm{E}+07$ \\
\hline $4,88 \mathrm{E}+07$ & $5,33 \mathrm{E}+07$ & $5,29 \mathrm{E}+07$ & $4,94 \mathrm{E}+07$ & $5,20 \mathrm{E}+07$ & $5,29 \mathrm{E}+07$ \\
\hline $4,91 \mathrm{E}+07$ & $5,34 \mathrm{E}+07$ & $5,30 \mathrm{E}+07$ & $5,00 \mathrm{E}+07$ & $5,21 \mathrm{E}+07$ & $5,28 \mathrm{E}+07$ \\
\hline $4,94 \mathrm{E}+07$ & $5,32 \mathrm{E}+07$ & $5,29 \mathrm{E}+07$ & $5,03 \mathrm{E}+07$ & $5,22 \mathrm{E}+07$ & $5,29 \mathrm{E}+07$ \\
\hline $4,96 \mathrm{E}+07$ & $5,72 \mathrm{E}+07$ & $5,27 \mathrm{E}+07$ & $5,06 \mathrm{E}+07$ & $5,20 \mathrm{E}+07$ & $5,33 \mathrm{E}+07$ \\
\hline $4,98 \mathrm{E}+07$ & $5,30 \mathrm{E}+07$ & $5,26 \mathrm{E}+07$ & $5,08 \mathrm{E}+07$ & $5,19 \mathrm{E}+07$ & $5,33 \mathrm{E}+07$ \\
\hline $5,00 \mathrm{E}+07$ & $5,28 \mathrm{E}+07$ & $5,26 \mathrm{E}+07$ & $5,09 \mathrm{E}+07$ & $5,18 \mathrm{E}+07$ & $5,33 \mathrm{E}+07$ \\
\hline $5,78 \mathrm{E}+07$ & $5,27 \mathrm{E}+07$ & $5,26 \mathrm{E}+07$ & $5,11 \mathrm{E}+07$ & $5,18 \mathrm{E}+07$ & $5,34 \mathrm{E}+07$ \\
\hline $5,06 \mathrm{E}+07$ & $5,27 \mathrm{E}+07$ & $5,27 \mathrm{E}+07$ & $5,13 \mathrm{E}+07$ & $5,18 \mathrm{E}+07$ & $5,36 \mathrm{E}+07$ \\
\hline $5,10 \mathrm{E}+07$ & $5,28 \mathrm{E}+07$ & $5,30 \mathrm{E}+07$ & $5,15 \mathrm{E}+07$ & $5,18 \mathrm{E}+07$ & $5,37 \mathrm{E}+07$ \\
\hline
\end{tabular}

Continua 
Continuação do Anexo E

\begin{tabular}{|c|c|c|c|c|c|}
\hline 31 & 32 & 33 & 34 & 35 & 36 \\
\hline $1,58 \mathrm{E}+06$ & $1,54 \mathrm{E}+06$ & $1,46 \mathrm{E}+06$ & $1,71 \mathrm{E}+06$ & $1,67 \mathrm{E}+06$ & $1,45 \mathrm{E}+06$ \\
\hline $3,62 \mathrm{E}+06$ & $5,02 \mathrm{E}+06$ & $5,28 \mathrm{E}+06$ & $6,55 \mathrm{E}+06$ & $5,79 \mathrm{E}+06$ & $4,77 \mathrm{E}+06$ \\
\hline $6,16 \mathrm{E}+06$ & $9,14 \mathrm{E}+06$ & $9,40 \mathrm{E}+06$ & $1,21 \mathrm{E}+07$ & $1,15 \mathrm{E}+07$ & $8,79 \mathrm{E}+06$ \\
\hline $1,08 \mathrm{E}+07$ & $1,42 \mathrm{E}+07$ & $1,36 \mathrm{E}+07$ & $1,77 \mathrm{E}+07$ & $1,81 \mathrm{E}+07$ & $1,39 \mathrm{E}+07$ \\
\hline $1,67 \mathrm{E}+07$ & $1,89 \mathrm{E}+07$ & $1,86 \mathrm{E}+07$ & $2,37 \mathrm{E}+07$ & $2,44 \mathrm{E}+07$ & $1,94 \mathrm{E}+07$ \\
\hline $2,31 \mathrm{E}+07$ & $2,54 \mathrm{E}+07$ & $2,39 \mathrm{E}+07$ & $2,88 \mathrm{E}+07$ & $3,12 \mathrm{E}+07$ & $2,50 \mathrm{E}+07$ \\
\hline $2,92 \mathrm{E}+07$ & $3,06 \mathrm{E}+07$ & $2,85 \mathrm{E}+07$ & $3,36 \mathrm{E}+07$ & $3,73 \mathrm{E}+07$ & $3,03 \mathrm{E}+07$ \\
\hline $3,51 \mathrm{E}+07$ & $3,54 \mathrm{E}+07$ & $3,32 \mathrm{E}+07$ & $3,71 \mathrm{E}+07$ & $4,23 \mathrm{E}+07$ & $3,52 \mathrm{E}+07$ \\
\hline $3,97 \mathrm{E}+07$ & $3,93 \mathrm{E}+07$ & $3,71 \mathrm{E}+07$ & $3,95 \mathrm{E}+07$ & $4,63 \mathrm{E}+07$ & $3,96 \mathrm{E}+07$ \\
\hline $4,38 \mathrm{E}+07$ & $4,25 \mathrm{E}+07$ & $4,09 \mathrm{E}+07$ & $4,13 \mathrm{E}+07$ & $4,93 \mathrm{E}+07$ & $4,28 \mathrm{E}+07$ \\
\hline $4,68 \mathrm{E}+07$ & $4,50 \mathrm{E}+07$ & $4,37 \mathrm{E}+07$ & $4,24 \mathrm{E}+07$ & $5,13 \mathrm{E}+07$ & $4,52 \mathrm{E}+07$ \\
\hline $4,92 \mathrm{E}+07$ & $4,67 \mathrm{E}+07$ & $4,60 \mathrm{E}+07$ & $4,31 \mathrm{E}+07$ & $5,26 \mathrm{E}+07$ & $4,72 \mathrm{E}+07$ \\
\hline $5,10 \mathrm{E}+07$ & $4,80 \mathrm{E}+07$ & $4,77 \mathrm{E}+07$ & $4,38 \mathrm{E}+07$ & $5,34 \mathrm{E}+07$ & $4,87 \mathrm{E}+07$ \\
\hline $5,22 \mathrm{E}+07$ & $4,90 \mathrm{E}+07$ & $4,92 \mathrm{E}+07$ & $4,45 \mathrm{E}+07$ & $5,37 \mathrm{E}+07$ & $4,97 \mathrm{E}+07$ \\
\hline $5,27 \mathrm{E}+07$ & $4,95 \mathrm{E}+07$ & $4,98 \mathrm{E}+07$ & $4,52 \mathrm{E}+07$ & $5,39 \mathrm{E}+07$ & $5,03 \mathrm{E}+07$ \\
\hline $5,32 \mathrm{E}+07$ & $4,97 \mathrm{E}+07$ & $5,03 \mathrm{E}+07$ & $4,59 \mathrm{E}+07$ & $5,39 \mathrm{E}+07$ & $5,05 \mathrm{E}+07$ \\
\hline $5,35 \mathrm{E}+07$ & $4,98 \mathrm{E}+07$ & $5,06 \mathrm{E}+07$ & $4,66 \mathrm{E}+07$ & $5,38 \mathrm{E}+07$ & $5,08 \mathrm{E}+07$ \\
\hline $5,38 \mathrm{E}+07$ & $4,98 \mathrm{E}+07$ & $5,07 \mathrm{E}+07$ & $4,73 \mathrm{E}+07$ & $5,37 \mathrm{E}+07$ & $5,09 \mathrm{E}+07$ \\
\hline $5,41 \mathrm{E}+07$ & $4,98 \mathrm{E}+07$ & $5,08 \mathrm{E}+07$ & $4,79 \mathrm{E}+07$ & $5,37 \mathrm{E}+07$ & $5,11 \mathrm{E}+07$ \\
\hline $5,42 \mathrm{E}+07$ & $4,98 \mathrm{E}+07$ & $5,09 \mathrm{E}+07$ & $4,86 \mathrm{E}+07$ & $5,37 \mathrm{E}+07$ & $5,11 \mathrm{E}+07$ \\
\hline $5,44 \mathrm{E}+07$ & $4,98 \mathrm{E}+07$ & $5,09 \mathrm{E}+07$ & $4,92 \mathrm{E}+07$ & $5,38 \mathrm{E}+07$ & $5,12 \mathrm{E}+07$ \\
\hline $5,46 \mathrm{E}+07$ & $4,98 \mathrm{E}+07$ & $5,10 \mathrm{E}+07$ & $4,96 \mathrm{E}+07$ & $5,39 \mathrm{E}+07$ & $5,12 \mathrm{E}+07$ \\
\hline $5,50 \mathrm{E}+07$ & $4,99 \mathrm{E}+07$ & $5,10 \mathrm{E}+07$ & $5,00 \mathrm{E}+07$ & $5,40 \mathrm{E}+07$ & \\
\hline
\end{tabular}

Continua 
Continuação do Anexo E

\begin{tabular}{|c|c|c|c|c|c|}
\hline 37 & 38 & 39 & 40 & 41 & 24 \\
\hline $1,45 \mathrm{E}+06$ & $1,48 \mathrm{E}+06$ & $1,59 \mathrm{E}+06$ & $1,59 \mathrm{E}+06$ & $1,24 \mathrm{E}+06$ & $1,58 \mathrm{E}+06$ \\
\hline $4,85 \mathrm{E}+06$ & $4,56 \mathrm{E}+06$ & $5,02 \mathrm{E}+06$ & $4,17 \mathrm{E}+06$ & $5,10 \mathrm{E}+06$ & $5,35 \mathrm{E}+06$ \\
\hline $8,84 \mathrm{E}+06$ & $8,14 \mathrm{E}+06$ & $8,73 \mathrm{E}+06$ & $6,72 \mathrm{E}+06$ & $1,02 \mathrm{E}+07$ & $1,08 \mathrm{E}+07$ \\
\hline $1,48 \mathrm{E}+07$ & $1,31 \mathrm{E}+07$ & $1,30 \mathrm{E}+07$ & $1,08 \mathrm{E}+07$ & $1,79 \mathrm{E}+07$ & $1,75 \mathrm{E}+07$ \\
\hline $2,08 \mathrm{E}+07$ & $1,86 \mathrm{E}+07$ & $1,74 \mathrm{E}+07$ & $1,58 \mathrm{E}+07$ & $2,17 \mathrm{E}+07$ & $2,30 \mathrm{E}+07$ \\
\hline $2,66 \mathrm{E}+07$ & $2,49 \mathrm{E}+07$ & $2,25 \mathrm{E}+07$ & $2,13 \mathrm{E}+07$ & $2,72 \mathrm{E}+07$ & $2,88 \mathrm{E}+07$ \\
\hline $3,18 \mathrm{E}+07$ & $3,08 \mathrm{E}+07$ & $2,71 \mathrm{E}+07$ & $2,67 \mathrm{E}+07$ & $3,26 \mathrm{E}+07$ & $3,40 \mathrm{E}+07$ \\
\hline $3,62 \mathrm{E}+07$ & $3,66 \mathrm{E}+07$ & $3,18 \mathrm{E}+07$ & $3,24 \mathrm{E}+07$ & $3,73 \mathrm{E}+07$ & $3,94 \mathrm{E}+07$ \\
\hline $4,02 \mathrm{E}+07$ & $4,14 \mathrm{E}+07$ & $3,60 \mathrm{E}+07$ & $3,80 \mathrm{E}+07$ & $4,09 \mathrm{E}+07$ & $4,20 \mathrm{E}+07$ \\
\hline $4,34 \mathrm{E}+07$ & $4,51 \mathrm{E}+07$ & $3,90 \mathrm{E}+07$ & $4,18 \mathrm{E}+07$ & $4,43 \mathrm{E}+07$ & $4,43 \mathrm{E}+07$ \\
\hline $4,61 \mathrm{E}+07$ & $4,78 \mathrm{E}+07$ & $4,23 \mathrm{E}+07$ & $4,47 \mathrm{E}+07$ & $4,68 \mathrm{E}+07$ & $4,58 \mathrm{E}+07$ \\
\hline $4,79 \mathrm{E}+07$ & $5,03 \mathrm{E}+07$ & $4,46 \mathrm{E}+07$ & $4,77 \mathrm{E}+07$ & $4,84 \mathrm{E}+07$ & $4,66 \mathrm{E}+07$ \\
\hline $4,90 \mathrm{E}+07$ & $5,18 \mathrm{E}+07$ & $4,65 \mathrm{E}+07$ & $4,96 \mathrm{E}+07$ & $4,96 \mathrm{E}+07$ & $4,71 \mathrm{E}+07$ \\
\hline $4,96 \mathrm{E}+07$ & $5,29 \mathrm{E}+07$ & $4,73 \mathrm{E}+07$ & $5,12 \mathrm{E}+07$ & $5,02 \mathrm{E}+07$ & $4,73 \mathrm{E}+07$ \\
\hline $5,00 \mathrm{E}+07$ & $5,35 \mathrm{E}+07$ & $4,87 \mathrm{E}+07$ & $5,21 \mathrm{E}+07$ & $5,05 \mathrm{E}+07$ & $4,73 \mathrm{E}+07$ \\
\hline $5,03 \mathrm{E}+07$ & $5,38 \mathrm{E}+07$ & $4,92 \mathrm{E}+07$ & $5,26 \mathrm{E}+07$ & $5,06 \mathrm{E}+07$ & $4,73 \mathrm{E}+07$ \\
\hline $5,04 \mathrm{E}+07$ & $5,40 \mathrm{E}+07$ & $4,95 \mathrm{E}+07$ & $5,30 \mathrm{E}+07$ & $5,07 \mathrm{E}+07$ & $4,74 \mathrm{E}+07$ \\
\hline $5,06 \mathrm{E}+07$ & $5,40 \mathrm{E}+07$ & $4,98 \mathrm{E}+07$ & $5,31 \mathrm{E}+07$ & $5,06 \mathrm{E}+07$ & $4,74 \mathrm{E}+07$ \\
\hline $5,07 \mathrm{E}+07$ & $5,41 \mathrm{E}+07$ & $4,99 \mathrm{E}+07$ & $5,32 \mathrm{E}+07$ & $5,04 \mathrm{E}+07$ & $4,74 \mathrm{E}+07$ \\
\hline $5,08 \mathrm{E}+07$ & $5,41 \mathrm{E}+07$ & $5,01 \mathrm{E}+07$ & $5,32 \mathrm{E}+07$ & $5,04 \mathrm{E}+07$ & $4,76 \mathrm{E}+07$ \\
\hline $5,10 \mathrm{E}+07$ & $5,41 \mathrm{E}+07$ & $5,01 \mathrm{E}+07$ & $5,32 \mathrm{E}+07$ & $5,05 \mathrm{E}+07$ & $4,79 \mathrm{E}+07$ \\
\hline $5,12 \mathrm{E}+07$ & $5,40 \mathrm{E}+07$ & $5,03 \mathrm{E}+07$ & $5,32 \mathrm{E}+07$ & $5,03 \mathrm{E}+07$ & $4,79 \mathrm{E}+07$ \\
\hline $5,15 \mathrm{E}+07$ & $5,41 \mathrm{E}+07$ & $5,04 \mathrm{E}+07$ & $5,34 \mathrm{E}+07$ & $5,04 \mathrm{E}+07$ & \\
\hline
\end{tabular}

Continua 
Continuação do Anexo E

\begin{tabular}{|c|c|c|c|c|r|}
\hline 43 & 44 & 45 & 46 & 48 & Mediana \\
\hline $1,38 \mathrm{E}+06$ & $1,53 \mathrm{E}+06$ & $1,37 \mathrm{E}+06$ & $1,39 \mathrm{E}+06$ & $1,38 \mathrm{E}+06$ & 1477,67 \\
\hline $4,73 \mathrm{E}+06$ & $4,32 \mathrm{E}+06$ & $3,88 \mathrm{E}+06$ & $4,92 \mathrm{E}+06$ & $5,40 \mathrm{E}+06$ & 4955,38 \\
\hline $9,76 \mathrm{E}+06$ & $7,49 \mathrm{E}+06$ & $7,49 \mathrm{E}+06$ & $9,04 \mathrm{E}+06$ & $1,07 \mathrm{E}+07$ & 9142,54 \\
\hline $1,58 \mathrm{E}+07$ & $1,26 \mathrm{E}+07$ & $1,24 \mathrm{E}+07$ & $1,51 \mathrm{E}+07$ & $1,70 \mathrm{E}+07$ & 14803,26 \\
\hline $2,14 \mathrm{E}+07$ & $1,87 \mathrm{E}+07$ & $1,75 \mathrm{E}+07$ & $2,07 \mathrm{E}+07$ & $2,27 \mathrm{E}+07$ & 20430,77 \\
\hline $2,70 \mathrm{E}+07$ & $2,50 \mathrm{E}+07$ & $2,27 \mathrm{E}+07$ & $2,58 \mathrm{E}+07$ & $2,80 \mathrm{E}+07$ & 25947,86 \\
\hline $3,21 \mathrm{E}+07$ & $3,03 \mathrm{E}+07$ & $2,76 \mathrm{E}+07$ & $3,10 \mathrm{E}+07$ & $3,28 \mathrm{E}+07$ & 31519,10 \\
\hline $3,64 \mathrm{E}+07$ & $3,48 \mathrm{E}+07$ & $3,18 \mathrm{E}+07$ & $3,53 \mathrm{E}+07$ & $3,65 \mathrm{E}+07$ & 36299,41 \\
\hline $4,00 \mathrm{E}+07$ & $3,87 \mathrm{E}+07$ & $3,54 \mathrm{E}+07$ & $3,91 \mathrm{E}+07$ & $3,99 \mathrm{E}+07$ & 40000,21 \\
\hline $4,27 \mathrm{E}+07$ & $4,19 \mathrm{E}+07$ & $3,86 \mathrm{E}+07$ & $4,24 \mathrm{E}+07$ & $4,21 \mathrm{E}+07$ & 43219,47 \\
\hline $4,49 \mathrm{E}+07$ & $4,49 \mathrm{E}+07$ & $4,09 \mathrm{E}+07$ & $4,46 \mathrm{E}+07$ & $4,39 \mathrm{E}+07$ & 45743,61 \\
\hline $4,63 \mathrm{E}+07$ & $4,49 \mathrm{E}+07$ & $4,28 \mathrm{E}+07$ & $4,65 \mathrm{E}+07$ & $4,50 \mathrm{E}+07$ & 47775,63 \\
\hline $4,72 \mathrm{E}+07$ & $4,88 \mathrm{E}+07$ & $4,43 \mathrm{E}+07$ & $4,78 \mathrm{E}+07$ & $4,57 \mathrm{E}+07$ & 49171,95 \\
\hline $4,77 \mathrm{E}+07$ & $5,03 \mathrm{E}+07$ & $4,51 \mathrm{E}+07$ & $4,87 \mathrm{E}+07$ & $4,62 \mathrm{E}+07$ & 49956,10 \\
\hline $4,80 \mathrm{E}+07$ & $5,11 \mathrm{E}+07$ & $4,59 \mathrm{E}+07$ & $4,92 \mathrm{E}+07$ & $4,65 \mathrm{E}+07$ & 50175,23 \\
\hline $4,82 \mathrm{E}+07$ & $5,20 \mathrm{E}+07$ & $4,65 \mathrm{E}+07$ & $4,94 \mathrm{E}+07$ & $4,67 \mathrm{E}+07$ & 50540,13 \\
\hline $4,85 \mathrm{E}+07$ & $5,22 \mathrm{E}+07$ & $4,69 \mathrm{E}+07$ & $4,95 \mathrm{E}+07$ & $4,68 \mathrm{E}+07$ & 50673,34 \\
\hline $4,87 \mathrm{E}+07$ & $5,25 \mathrm{E}+07$ & $4,73 \mathrm{E}+07$ & $4,94 \mathrm{E}+07$ & $4,70 \mathrm{E}+07$ & 50756,62 \\
\hline $4,90 \mathrm{E}+07$ & $5,26 \mathrm{E}+07$ & $4,76 \mathrm{E}+07$ & $4,94 \mathrm{E}+07$ & $4,72 \mathrm{E}+07$ & 50864,60 \\
\hline $4,93 \mathrm{E}+07$ & $5,28 \mathrm{E}+07$ & $4,78 \mathrm{E}+07$ & $4,95 \mathrm{E}+07$ & $4,75 \mathrm{E}+07$ & 51191,46 \\
\hline $4,96 \mathrm{E}+07$ & $5,30 \mathrm{E}+07$ & $4,80 \mathrm{E}+07$ & $4,95 \mathrm{E}+07$ & $4,77 \mathrm{E}+07$ & 51653,53 \\
\hline $4,99 \mathrm{E}+07$ & $5,32 \mathrm{E}+07$ & $4,82 \mathrm{E}+07$ & $4,95 \mathrm{E}+07$ & $4,79 \mathrm{E}+07$ & 51699,98 \\
\hline $5,03 \mathrm{E}+07$ & $5,34 \mathrm{E}+07$ & $4,84 \mathrm{E}+07$ & $4,96 \mathrm{E}+07$ & $4,82 \mathrm{E}+07$ & 51870,87 \\
\hline
\end{tabular}


ANEXO F

DADOS DOS CORPOS DE PROVA DO GRUPO 2M 


\begin{tabular}{|c|c|c|c|c|c|c|}
\hline & $\mathbf{1}$ & $\mathbf{2}$ & $\mathbf{3}$ & $\mathbf{4}$ & $\mathbf{5}$ & $\mathbf{6}$ \\
\hline 0,00 & $1,56 \mathrm{E}+06$ & $1,57 \mathrm{E}+06$ & $1,63 \mathrm{E}+06$ & $1,63 \mathrm{E}+06$ & $1,68 \mathrm{E}+06$ & $1,67 \mathrm{E}+06$ \\
\hline 0,05 & $5,60 \mathrm{E}+06$ & $4,92 \mathrm{E}+06$ & $5,07 \mathrm{E}+06$ & $5,03 \mathrm{E}+06$ & $5,40 \mathrm{E}+06$ & $6,21 \mathrm{E}+06$ \\
\hline 0,10 & $9,95 \mathrm{E}+06$ & $8,51 \mathrm{E}+06$ & $9,71 \mathrm{E}+06$ & $9,24 \mathrm{E}+06$ & $1,04 \mathrm{E}+07$ & $1,37 \mathrm{E}+07$ \\
\hline 0,15 & $1,68 \mathrm{E}+07$ & $1,56 \mathrm{E}+07$ & $1,57 \mathrm{E}+07$ & $1,52 \mathrm{E}+07$ & $1,80 \mathrm{E}+07$ & $1,97 \mathrm{E}+07$ \\
\hline 0,20 & $2,33 \mathrm{E}+07$ & $2,25 \mathrm{E}+07$ & $2,29 \mathrm{E}+07$ & $2,19 \mathrm{E}+07$ & $2,51 \mathrm{E}+07$ & $2,64 \mathrm{E}+07$ \\
\hline 0,25 & $3,00 \mathrm{E}+07$ & $2,93 \mathrm{E}+07$ & $2,94 \mathrm{E}+07$ & $2,84 \mathrm{E}+07$ & $3,23 \mathrm{E}+07$ & $3,32 \mathrm{E}+07$ \\
\hline 0,30 & $3,61 \mathrm{E}+07$ & $3,51 \mathrm{E}+07$ & $3,49 \mathrm{E}+07$ & $3,47 \mathrm{E}+07$ & $3,94 \mathrm{E}+07$ & $3,90 \mathrm{E}+07$ \\
\hline 0,35 & $4,12 \mathrm{E}+07$ & $4,06 \mathrm{E}+07$ & $4,01 \mathrm{E}+07$ & $4,06 \mathrm{E}+07$ & $4,56 \mathrm{E}+07$ & $4,46 \mathrm{E}+07$ \\
\hline 0,40 & $4,57 \mathrm{E}+07$ & $4,51 \mathrm{E}+07$ & $4,48 \mathrm{E}+07$ & $4,55 \mathrm{E}+07$ & $5,06 \mathrm{E}+07$ & $4,94 \mathrm{E}+07$ \\
\hline 0,45 & $4,90 \mathrm{E}+07$ & $4,94 \mathrm{E}+07$ & $4,84 \mathrm{E}+07$ & $5,03 \mathrm{E}+07$ & $5,53 \mathrm{E}+07$ & $5,34 \mathrm{E}+07$ \\
\hline 0,50 & $5,14 \mathrm{E}+07$ & $5,26 \mathrm{E}+07$ & $5,10 \mathrm{E}+07$ & $5,36 \mathrm{E}+07$ & $5,83 \mathrm{E}+07$ & $5,63 \mathrm{E}+07$ \\
\hline 0,55 & $5,29 \mathrm{E}+07$ & $5,48 \mathrm{E}+07$ & $5,28 \mathrm{E}+07$ & $5,60 \mathrm{E}+07$ & $6,02 \mathrm{E}+07$ & $5,83 \mathrm{E}+07$ \\
\hline 0,60 & $5,38 \mathrm{E}+07$ & $5,63 \mathrm{E}+07$ & $5,41 \mathrm{E}+07$ & $5,76 \mathrm{E}+07$ & $6,13 \mathrm{E}+07$ & $5,95 \mathrm{E}+07$ \\
\hline 0,65 & $5,44 \mathrm{E}+07$ & $5,71 \mathrm{E}+07$ & $5,48 \mathrm{E}+07$ & $5,84 \mathrm{E}+07$ & $6,19 \mathrm{E}+07$ & $6,02 \mathrm{E}+07$ \\
\hline 0,70 & $5,48 \mathrm{E}+07$ & $5,73 \mathrm{E}+07$ & $5,52 \mathrm{E}+07$ & $5,88 \mathrm{E}+07$ & $6,19 \mathrm{E}+07$ & $6,04 \mathrm{E}+07$ \\
\hline 0,75 & $5,49 \mathrm{E}+07$ & $5,74 \mathrm{E}+07$ & $5,56 \mathrm{E}+07$ & $5,90 \mathrm{E}+07$ & $6,19 \mathrm{E}+07$ & $6,04 \mathrm{E}+07$ \\
\hline 0,80 & $5,51 \mathrm{E}+07$ & $5,72 \mathrm{E}+07$ & $5,61 \mathrm{E}+07$ & $5,91 \mathrm{E}+07$ & $6,17 \mathrm{E}+07$ & $6,04 \mathrm{E}+07$ \\
\hline 0,85 & $5,53 \mathrm{E}+07$ & $5,71 \mathrm{E}+07$ & $5,66 \mathrm{E}+07$ & $5,92 \mathrm{E}+07$ & $6,16 \mathrm{E}+07$ & $6,04 \mathrm{E}+07$ \\
\hline 0,90 & $5,54 \mathrm{E}+07$ & $5,71 \mathrm{E}+07$ & $5,71 \mathrm{E}+07$ & $5,93 \mathrm{E}+07$ & $6,15 \mathrm{E}+07$ & $6,03 \mathrm{E}+07$ \\
\hline 0,95 & $5,56 \mathrm{E}+07$ & $5,71 \mathrm{E}+07$ & $5,77 \mathrm{E}+07$ & $5,95 \mathrm{E}+07$ & $6,15 \mathrm{E}+07$ & $6,04 \mathrm{E}+07$ \\
\hline 1,00 & $5,59 \mathrm{E}+07$ & $5,73 \mathrm{E}+07$ & $5,82 \mathrm{E}+07$ & $5,97 \mathrm{E}+07$ & $6,16 \mathrm{E}+07$ & \\
\hline 1,05 & & & $5,88 \mathrm{E}+07$ & & & \\
\hline 1,10 & & & $5,86 \mathrm{E}+07$ & & & \\
\hline 1,15 & & & $5,99 \mathrm{E}+07$ & & & \\
\hline 1,20 & & & $6,03 \mathrm{E}+07$ & & & \\
\hline 1,25 & & & $6,08 \mathrm{E}+07$ & & & \\
\hline
\end{tabular}

Continua 
Continuação do Anexo F

\begin{tabular}{|c|c|c|c|c|c|c|}
\hline $\mathbf{7}$ & $\mathbf{8}$ & $\mathbf{9}$ & $\mathbf{1 0}$ & $\mathbf{1 1}$ & $\mathbf{1 2}$ & $\mathbf{1 3}$ \\
\hline $1,72 \mathrm{E}+06$ & $1,53 \mathrm{E}+06$ & $1,78 \mathrm{E}+06$ & $1,49 \mathrm{E}+06$ & $1,50 \mathrm{E}+06$ & $1,42 \mathrm{E}+06$ & $1,52 \mathrm{E}+06$ \\
\hline $5,95 \mathrm{E}+06$ & $5,24 \mathrm{E}+06$ & $5,75 \mathrm{E}+06$ & $5,62 \mathrm{E}+06$ & $4,71 \mathrm{E}+06$ & $3,83 \mathrm{E}+06$ & $4,07 \mathrm{E}+06$ \\
\hline $1,06 \mathrm{E}+07$ & $1,04 \mathrm{E}+07$ & $1,12 \mathrm{E}+07$ & $1,06 \mathrm{E}+07$ & $8,96 \mathrm{E}+06$ & $8,03 \mathrm{E}+06$ & $7,39 \mathrm{E}+06$ \\
\hline $1,79 \mathrm{E}+07$ & $1,79 \mathrm{E}+07$ & $1,85 \mathrm{E}+07$ & $1,71 \mathrm{E}+07$ & $1,51 \mathrm{E}+07$ & $1,44 \mathrm{E}+07$ & $1,33 \mathrm{E}+07$ \\
\hline $2,56 \mathrm{E}+07$ & $2,48 \mathrm{E}+07$ & $2,60 \mathrm{E}+07$ & $2,36 \mathrm{E}+07$ & $2,21 \mathrm{E}+07$ & $2,10 \mathrm{E}+07$ & $2,02 \mathrm{E}+07$ \\
\hline $3,30 \mathrm{E}+07$ & $3,16 \mathrm{E}+07$ & $3,43 \mathrm{E}+07$ & $3,07 \mathrm{E}+07$ & $2,90 \mathrm{E}+07$ & $2,78 \mathrm{E}+07$ & $2,67 \mathrm{E}+07$ \\
\hline $3,96 \mathrm{E}+07$ & $3,77 \mathrm{E}+07$ & $4,07 \mathrm{E}+07$ & $3,70 \mathrm{E}+07$ & $3,55 \mathrm{E}+07$ & $3,40 \mathrm{E}+07$ & $3,26 \mathrm{E}+07$ \\
\hline $4,53 \mathrm{E}+07$ & $4,37 \mathrm{E}+07$ & $4,71 \mathrm{E}+07$ & $4,29 \mathrm{E}+07$ & $4,16 \mathrm{E}+07$ & $3,92 \mathrm{E}+07$ & $3,79 \mathrm{E}+07$ \\
\hline $5,03 \mathrm{E}+07$ & $4,89 \mathrm{E}+07$ & $5,20 \mathrm{E}+07$ & $4,87 \mathrm{E}+07$ & $4,64 \mathrm{E}+07$ & $4,47 \mathrm{E}+07$ & $4,34 \mathrm{E}+07$ \\
\hline $5,48 \mathrm{E}+07$ & $5,34 \mathrm{E}+07$ & $5,65 \mathrm{E}+07$ & $5,27 \mathrm{E}+07$ & $5,14 \mathrm{E}+07$ & $4,85 \mathrm{E}+07$ & $4,79 \mathrm{E}+07$ \\
\hline $5,81 \mathrm{E}+07$ & $5,65 \mathrm{E}+07$ & $5,97 \mathrm{E}+07$ & $5,59 \mathrm{E}+07$ & $5,49 \mathrm{E}+07$ & $5,16 \mathrm{E}+07$ & $5,14 \mathrm{E}+07$ \\
\hline $6,02 \mathrm{E}+07$ & $5,88 \mathrm{E}+07$ & $6,17 \mathrm{E}+07$ & $5,81 \mathrm{E}+07$ & $5,75 \mathrm{E}+07$ & $5,36 \mathrm{E}+07$ & $5,45 \mathrm{E}+07$ \\
\hline $6,15 \mathrm{E}+07$ & $6,02 \mathrm{E}+07$ & $6,31 \mathrm{E}+07$ & $5,94 \mathrm{E}+07$ & $5,92 \mathrm{E}+07$ & $5,47 \mathrm{E}+07$ & $5,68 \mathrm{E}+07$ \\
\hline $6,22 \mathrm{E}+07$ & $6,10 \mathrm{E}+07$ & $6,38 \mathrm{E}+07$ & $6,01 \mathrm{E}+07$ & $6,01 \mathrm{E}+07$ & $5,52 \mathrm{E}+07$ & $5,84 \mathrm{E}+07$ \\
\hline $6,23 \mathrm{E}+07$ & $6,13 \mathrm{E}+07$ & $6,42 \mathrm{E}+07$ & $6,05 \mathrm{E}+07$ & $6,04 \mathrm{E}+07$ & $5,53 \mathrm{E}+07$ & $5,94 \mathrm{E}+07$ \\
\hline $6,22 \mathrm{E}+07$ & $6,14 \mathrm{E}+07$ & $6,45 \mathrm{E}+07$ & $6,07 \mathrm{E}+07$ & $6,04 \mathrm{E}+07$ & $5,52 \mathrm{E}+07$ & $6,03 \mathrm{E}+07$ \\
\hline $6,20 \mathrm{E}+07$ & $6,14 \mathrm{E}+07$ & $6,48 \mathrm{E}+07$ & $6,07 \mathrm{E}+07$ & $6,03 \mathrm{E}+07$ & $5,51 \mathrm{E}+07$ & $6,06 \mathrm{E}+07$ \\
\hline $6,19 \mathrm{E}+07$ & $6,13 \mathrm{E}+07$ & $6,49 \mathrm{E}+07$ & $6,06 \mathrm{E}+07$ & $6,02 \mathrm{E}+07$ & $5,49 \mathrm{E}+07$ & $6,08 \mathrm{E}+07$ \\
\hline $6,18 \mathrm{E}+07$ & $6,11 \mathrm{E}+07$ & $6,52 \mathrm{E}+07$ & $6,06 \mathrm{E}+07$ & $6,01 \mathrm{E}+07$ & $5,49 \mathrm{E}+07$ & $6,09 \mathrm{E}+07$ \\
\hline $6,18 \mathrm{E}+07$ & $6,11 \mathrm{E}+07$ & $6,54 \mathrm{E}+07$ & $6,06 \mathrm{E}+07$ & $6,00 \mathrm{E}+07$ & $5,50 \mathrm{E}+07$ & $6,09 \mathrm{E}+07$ \\
\hline $6,19 \mathrm{E}+07$ & $6,11 \mathrm{E}+07$ & $6,57 \mathrm{E}+07$ & $6,07 \mathrm{E}+07$ & $6,01 \mathrm{E}+07$ & $5,52 \mathrm{E}+07$ & $6,10 \mathrm{E}+07$ \\
\hline & & & & & & $6,10 \mathrm{E}+07$ \\
\hline & & & & & & $6,09 \mathrm{E}+07$ \\
\hline & & & & & & $6,08 \mathrm{E}+07$ \\
\hline & & & & & & $6,09 \mathrm{E}+07$ \\
\hline & & & & & & \\
\hline
\end{tabular}

Continua 
Continuação do Anexo F

\begin{tabular}{|c|c|c|c|c|c|c|}
\hline $\mathbf{1 4}$ & $\mathbf{1 5}$ & $\mathbf{1 6}$ & $\mathbf{1 7}$ & $\mathbf{1 8}$ & $\mathbf{1 9}$ & $\mathbf{2 0}$ \\
\hline $1,73 \mathrm{E}+06$ & $1,74 \mathrm{E}+06$ & $1,47 \mathrm{E}+06$ & $1,64 \mathrm{E}+06$ & $1,66 \mathrm{E}+06$ & $1,61 \mathrm{E}+06$ & $1,59 \mathrm{E}+06$ \\
\hline $6,34 \mathrm{E}+06$ & $6,82 \mathrm{E}+06$ & $8,14 \mathrm{E}+06$ & $5,52 \mathrm{E}+06$ & $5,64 \mathrm{E}+06$ & $6,33 \mathrm{E}+06$ & $4,94 \mathrm{E}+06$ \\
\hline $1,16 \mathrm{E}+07$ & $1,20 \mathrm{E}+07$ & $1,29 \mathrm{E}+07$ & $1,19 \mathrm{E}+07$ & $1,10 \mathrm{E}+07$ & $1,21 \mathrm{E}+07$ & $9,53 \mathrm{E}+06$ \\
\hline $1,93 \mathrm{E}+07$ & $1,86 \mathrm{E}+07$ & $1,86 \mathrm{E}+07$ & $1,91 \mathrm{E}+07$ & $1,79 \mathrm{E}+07$ & $2,01 \mathrm{E}+07$ & $1,56 \mathrm{E}+07$ \\
\hline $2,68 \mathrm{E}+07$ & $2,62 \mathrm{E}+07$ & $2,51 \mathrm{E}+07$ & $2,62 \mathrm{E}+07$ & $2,51 \mathrm{E}+07$ & $2,66 \mathrm{E}+07$ & $2,24 \mathrm{E}+07$ \\
\hline $3,42 \mathrm{E}+07$ & $3,31 \mathrm{E}+07$ & $3,07 \mathrm{E}+07$ & $3,38 \mathrm{E}+07$ & $3,22 \mathrm{E}+07$ & $3,34 \mathrm{E}+07$ & $2,90 \mathrm{E}+07$ \\
\hline $4,07 \mathrm{E}+07$ & $3,91 \mathrm{E}+07$ & $3,60 \mathrm{E}+07$ & $4,00 \mathrm{E}+07$ & $3,83 \mathrm{E}+07$ & $3,97 \mathrm{E}+07$ & $3,57 \mathrm{E}+07$ \\
\hline $4,73 \mathrm{E}+07$ & $4,49 \mathrm{E}+07$ & $4,13 \mathrm{E}+07$ & $4,59 \mathrm{E}+07$ & $4,34 \mathrm{E}+07$ & $4,53 \mathrm{E}+07$ & $4,15 \mathrm{E}+07$ \\
\hline $5,29 \mathrm{E}+07$ & $4,99 \mathrm{E}+07$ & $4,57 \mathrm{E}+07$ & $5,14 \mathrm{E}+07$ & $4,84 \mathrm{E}+07$ & $4,94 \mathrm{E}+07$ & $4,65 \mathrm{E}+07$ \\
\hline $5,72 \mathrm{E}+07$ & $5,40 \mathrm{E}+07$ & $5,03 \mathrm{E}+07$ & $5,53 \mathrm{E}+07$ & $5,21 \mathrm{E}+07$ & $5,29 \mathrm{E}+07$ & $5,17 \mathrm{E}+07$ \\
\hline $6,02 \mathrm{E}+07$ & $5,67 \mathrm{E}+07$ & $5,40 \mathrm{E}+07$ & $5,79 \mathrm{E}+07$ & $5,45 \mathrm{E}+07$ & $5,58 \mathrm{E}+07$ & $5,54 \mathrm{E}+07$ \\
\hline $6,22 \mathrm{E}+07$ & $5,87 \mathrm{E}+07$ & $5,69 \mathrm{E}+07$ & $5,96 \mathrm{E}+07$ & $5,61 \mathrm{E}+07$ & $5,76 \mathrm{E}+07$ & $5,77 \mathrm{E}+07$ \\
\hline $6,34 \mathrm{E}+07$ & $5,97 \mathrm{E}+07$ & $5,90 \mathrm{E}+07$ & $6,05 \mathrm{E}+07$ & $5,70 \mathrm{E}+07$ & $5,88 \mathrm{E}+07$ & $5,93 \mathrm{E}+07$ \\
\hline $6,39 \mathrm{E}+07$ & $6,03 \mathrm{E}+07$ & $6,03 \mathrm{E}+07$ & $6,09 \mathrm{E}+07$ & $5,74 \mathrm{E}+07$ & $5,95 \mathrm{E}+07$ & $6,01 \mathrm{E}+07$ \\
\hline $6,41 \mathrm{E}+07$ & $6,06 \mathrm{E}+07$ & $6,11 \mathrm{E}+07$ & $6,11 \mathrm{E}+07$ & $5,75 \mathrm{E}+07$ & $5,98 \mathrm{E}+07$ & $6,05 \mathrm{E}+07$ \\
\hline $6,41 \mathrm{E}+07$ & $6,08 \mathrm{E}+07$ & $6,15 \mathrm{E}+07$ & $6,11 \mathrm{E}+07$ & $5,76 \mathrm{E}+07$ & $5,98 \mathrm{E}+07$ & $6,05 \mathrm{E}+07$ \\
\hline $6,40 \mathrm{E}+07$ & $6,08 \mathrm{E}+07$ & $6,16 \mathrm{E}+07$ & $6,11 \mathrm{E}+07$ & $5,76 \mathrm{E}+07$ & $5,97 \mathrm{E}+07$ & $6,05 \mathrm{E}+07$ \\
\hline $6,38 \mathrm{E}+07$ & $6,09 \mathrm{E}+07$ & $6,16 \mathrm{E}+07$ & $6,12 \mathrm{E}+07$ & $5,77 \mathrm{E}+07$ & $5,96 \mathrm{E}+07$ & $6,05 \mathrm{E}+07$ \\
\hline $6,37 \mathrm{E}+07$ & $6,10 \mathrm{E}+07$ & $6,15 \mathrm{E}+07$ & $6,13 \mathrm{E}+07$ & $5,77 \mathrm{E}+07$ & $5,96 \mathrm{E}+07$ & $6,05 \mathrm{E}+07$ \\
\hline $6,37 \mathrm{E}+07$ & $6,13 \mathrm{E}+07$ & $6,14 \mathrm{E}+07$ & $6,16 \mathrm{E}+07$ & $5,78 \mathrm{E}+07$ & $5,96 \mathrm{E}+07$ & $6,05 \mathrm{E}+07$ \\
\hline $6,36 \mathrm{E}+07$ & $6,15 \mathrm{E}+07$ & & $6,19 \mathrm{E}+07$ & $5,79 \mathrm{E}+07$ & $5,96 \mathrm{E}+07$ & $6,06 \mathrm{E}+07$ \\
\hline & & & & & & \\
\hline & & & & & & \\
\hline & & & & & & \\
\hline & & & & & & \\
\hline
\end{tabular}

Continua 


\section{Continuação do Anexo F}

\begin{tabular}{|c|c|c|c|c|c|c|}
\hline $\mathbf{2 1}$ & $\mathbf{2 2}$ & $\mathbf{2 3}$ & $\mathbf{2 4}$ & $\mathbf{2 5}$ & $\mathbf{2 6}$ & $\mathbf{2 7}$ \\
\hline $1,48 \mathrm{E}+06$ & $1,61 \mathrm{E}+06$ & $1,65 \mathrm{E}+06$ & $1,51 \mathrm{E}+06$ & $1,58 \mathrm{E}+06$ & $1,55 \mathrm{E}+06$ & $1,58 \mathrm{E}+06$ \\
\hline $5,63 \mathrm{E}+06$ & $5,08 \mathrm{E}+06$ & $5,37 \mathrm{E}+06$ & $4,74 \mathrm{E}+06$ & $5,13 \mathrm{E}+06$ & $4,87 \mathrm{E}+06$ & $5,45 \mathrm{E}+06$ \\
\hline $1,01 \mathrm{E}+07$ & $9,94 \mathrm{E}+06$ & $9,58 \mathrm{E}+06$ & $9,04 \mathrm{E}+06$ & $1,05 \mathrm{E}+07$ & $8,94 \mathrm{E}+06$ & $9,45 \mathrm{E}+06$ \\
\hline $1,66 \mathrm{E}+07$ & $1,62 \mathrm{E}+07$ & $1,61 \mathrm{E}+07$ & $1,59 \mathrm{E}+07$ & $1,73 \mathrm{E}+07$ & $1,48 \mathrm{E}+07$ & $1,56 \mathrm{E}+07$ \\
\hline $2,33 \mathrm{E}+07$ & $2,31 \mathrm{E}+07$ & $2,31 \mathrm{E}+07$ & $2,31 \mathrm{E}+07$ & $2,47 \mathrm{E}+07$ & $2,08 \mathrm{E}+07$ & $2,17 \mathrm{E}+07$ \\
\hline $2,96 \mathrm{E}+07$ & $3,03 \mathrm{E}+07$ & $3,02 \mathrm{E}+07$ & $2,98 \mathrm{E}+07$ & $3,00 \mathrm{E}+07$ & $2,67 \mathrm{E}+07$ & $2,75 \mathrm{E}+07$ \\
\hline $3,55 \mathrm{E}+07$ & $3,72 \mathrm{E}+07$ & $3,58 \mathrm{E}+07$ & $3,65 \mathrm{E}+07$ & $3,52 \mathrm{E}+07$ & $3,23 \mathrm{E}+07$ & $3,18 \mathrm{E}+07$ \\
\hline $4,14 \mathrm{E}+07$ & $4,31 \mathrm{E}+07$ & $4,11 \mathrm{E}+07$ & $4,21 \mathrm{E}+07$ & $3,95 \mathrm{E}+07$ & $3,74 \mathrm{E}+07$ & $3,58 \mathrm{E}+07$ \\
\hline $4,62 \mathrm{E}+07$ & $4,83 \mathrm{E}+07$ & $4,58 \mathrm{E}+07$ & $4,72 \mathrm{E}+07$ & $4,31 \mathrm{E}+07$ & $4,19 \mathrm{E}+07$ & $4,04 \mathrm{E}+07$ \\
\hline $4,96 \mathrm{E}+07$ & $5,30 \mathrm{E}+07$ & $4,94 \mathrm{E}+07$ & $5,16 \mathrm{E}+07$ & $4,58 \mathrm{E}+07$ & $4,59 \mathrm{E}+07$ & $4,45 \mathrm{E}+07$ \\
\hline $5,21 \mathrm{E}+07$ & $5,62 \mathrm{E}+07$ & $5,28 \mathrm{E}+07$ & $5,47 \mathrm{E}+07$ & $4,79 \mathrm{E}+07$ & $4,95 \mathrm{E}+07$ & $4,87 \mathrm{E}+07$ \\
\hline $5,37 \mathrm{E}+07$ & $5,84 \mathrm{E}+07$ & $5,48 \mathrm{E}+07$ & $5,67 \mathrm{E}+07$ & $4,94 \mathrm{E}+07$ & $5,22 \mathrm{E}+07$ & $5,18 \mathrm{E}+07$ \\
\hline $5,46 \mathrm{E}+07$ & $5,99 \mathrm{E}+07$ & $5,62 \mathrm{E}+07$ & $5,82 \mathrm{E}+07$ & $5,05 \mathrm{E}+07$ & $5,43 \mathrm{E}+07$ & $5,48 \mathrm{E}+07$ \\
\hline $5,51 \mathrm{E}+07$ & $6,06 \mathrm{E}+07$ & $5,71 \mathrm{E}+07$ & $5,90 \mathrm{E}+07$ & $5,15 \mathrm{E}+07$ & $5,56 \mathrm{E}+07$ & $5,73 \mathrm{E}+07$ \\
\hline $5,52 \mathrm{E}+07$ & $6,10 \mathrm{E}+07$ & $5,75 \mathrm{E}+07$ & $5,94 \mathrm{E}+07$ & $5,21 \mathrm{E}+07$ & $5,65 \mathrm{E}+07$ & $5,94 \mathrm{E}+07$ \\
\hline $5,52 \mathrm{E}+07$ & $6,13 \mathrm{E}+07$ & $5,78 \mathrm{E}+07$ & $5,95 \mathrm{E}+07$ & $5,26 \mathrm{E}+07$ & $5,71 \mathrm{E}+07$ & $6,12 \mathrm{E}+07$ \\
\hline $5,52 \mathrm{E}+07$ & $6,15 \mathrm{E}+07$ & $5,79 \mathrm{E}+07$ & $5,95 \mathrm{E}+07$ & $5,31 \mathrm{E}+07$ & $5,74 \mathrm{E}+07$ & $6,26 \mathrm{E}+07$ \\
\hline $5,52 \mathrm{E}+07$ & $6,17 \mathrm{E}+07$ & $5,81 \mathrm{E}+07$ & $5,95 \mathrm{E}+07$ & $5,35 \mathrm{E}+07$ & $5,76 \mathrm{E}+07$ & $6,36 \mathrm{E}+07$ \\
\hline $5,52 \mathrm{E}+07$ & $6,20 \mathrm{E}+07$ & $5,84 \mathrm{E}+07$ & $5,96 \mathrm{E}+07$ & $5,40 \mathrm{E}+07$ & $5,78 \mathrm{E}+07$ & $6,43 \mathrm{E}+07$ \\
\hline $5,53 \mathrm{E}+07$ & $6,22 \mathrm{E}+07$ & $5,87 \mathrm{E}+07$ & $5,97 \mathrm{E}+07$ & $5,43 \mathrm{E}+07$ & $5,79 \mathrm{E}+07$ & $6,47 \mathrm{E}+07$ \\
\hline $5,53 \mathrm{E}+07$ & $6,26 \mathrm{E}+07$ & $5,91 \mathrm{E}+07$ & $5,97 \mathrm{E}+07$ & $5,47 \mathrm{E}+07$ & $5,81 \mathrm{E}+07$ & $6,51 \mathrm{E}+07$ \\
\hline & $6,30 \mathrm{E}+07$ & $5,95 \mathrm{E}+07$ & $6,00 \mathrm{E}+07$ & $5,51 \mathrm{E}+07$ & $5,84 \mathrm{E}+07$ & $6,52 \mathrm{E}+07$ \\
\hline & $6,34 \mathrm{E}+07$ & $5,95 \mathrm{E}+07$ & $6,02 \mathrm{E}+07$ & $5,60 \mathrm{E}+07$ & $5,87 \mathrm{E}+07$ & $6,53 \mathrm{E}+07$ \\
\hline & & & & $5,66 \mathrm{E}+07$ & 6 & $6,53 \mathrm{E}+07$ \\
\hline & & & & & & $6,52 \mathrm{E}+07$ \\
\hline & & & & & $6,52 \mathrm{E}+07$ \\
\hline
\end{tabular}

Continua 


\section{Continuação do Anexo F}

\begin{tabular}{|c|c|c|c|c|c|c|}
\hline $\mathbf{2 8}$ & $\mathbf{2 9}$ & $\mathbf{3 0}$ & $\mathbf{3 1}$ & $\mathbf{3 2}$ & $\mathbf{3 3}$ & $\mathbf{3 4}$ \\
\hline $1,59 \mathrm{E}+06$ & $1,57 \mathrm{E}+06$ & $1,56 \mathrm{E}+06$ & $1,51 \mathrm{E}+06$ & $1,53 \mathrm{E}+06$ & $1,49 \mathrm{E}+06$ & $1,50 \mathrm{E}+06$ \\
\hline $5,48 \mathrm{E}+06$ & $6,65 \mathrm{E}+06$ & $6,38 \mathrm{E}+06$ & $4,96 \mathrm{E}+06$ & $1,18 \mathrm{E}+07$ & $1,02 \mathrm{E}+07$ & $4,21 \mathrm{E}+06$ \\
\hline $1,04 \mathrm{E}+07$ & $1,18 \mathrm{E}+07$ & $1,21 \mathrm{E}+07$ & $9,34 \mathrm{E}+06$ & $1,86 \mathrm{E}+07$ & $1,68 \mathrm{E}+07$ & $7,71 \mathrm{E}+06$ \\
\hline $1,68 \mathrm{E}+07$ & $1,97 \mathrm{E}+07$ & $2,02 \mathrm{E}+07$ & $1,52 \mathrm{E}+07$ & $2,57 \mathrm{E}+07$ & $2,39 \mathrm{E}+07$ & $1,31 \mathrm{E}+07$ \\
\hline $2,84 \mathrm{E}+07$ & $2,63 \mathrm{E}+07$ & $2,80 \mathrm{E}+07$ & $2,13 \mathrm{E}+07$ & $3,21 \mathrm{E}+07$ & $3,07 \mathrm{E}+07$ & $1,88 \mathrm{E}+07$ \\
\hline $3,58 \mathrm{E}+07$ & $3,38 \mathrm{E}+07$ & $3,55 \mathrm{E}+07$ & $2,80 \mathrm{E}+07$ & $3,78 \mathrm{E}+07$ & $3,69 \mathrm{E}+07$ & $2,50 \mathrm{E}+07$ \\
\hline $4,15 \mathrm{E}+07$ & $3,95 \mathrm{E}+07$ & $4,21 \mathrm{E}+07$ & $3,41 \mathrm{E}+07$ & $4,31 \mathrm{E}+07$ & $4,24 \mathrm{E}+07$ & $3,10 \mathrm{E}+07$ \\
\hline $4,54 \mathrm{E}+07$ & $4,47 \mathrm{E}+07$ & $4,89 \mathrm{E}+07$ & $3,97 \mathrm{E}+07$ & $4,73 \mathrm{E}+07$ & $4,75 \mathrm{E}+07$ & $3,67 \mathrm{E}+07$ \\
\hline $4,98 \mathrm{E}+07$ & $4,95 \mathrm{E}+07$ & $5,42 \mathrm{E}+07$ & $4,48 \mathrm{E}+07$ & $5,10 \mathrm{E}+07$ & $5,14 \mathrm{E}+07$ & $4,17 \mathrm{E}+07$ \\
\hline $5,29 \mathrm{E}+07$ & $5,33 \mathrm{E}+07$ & $5,88 \mathrm{E}+07$ & $4,91 \mathrm{E}+07$ & $5,38 \mathrm{E}+07$ & $5,46 \mathrm{E}+07$ & $4,64 \mathrm{E}+07$ \\
\hline $5,51 \mathrm{E}+07$ & $5,57 \mathrm{E}+07$ & $6,20 \mathrm{E}+07$ & $5,20 \mathrm{E}+07$ & $5,56 \mathrm{E}+07$ & $5,69 \mathrm{E}+07$ & $5,04 \mathrm{E}+07$ \\
\hline $5,66 \mathrm{E}+07$ & $5,72 \mathrm{E}+07$ & $6,42 \mathrm{E}+07$ & $5,44 \mathrm{E}+07$ & $5,68 \mathrm{E}+07$ & $5,84 \mathrm{E}+07$ & $5,33 \mathrm{E}+07$ \\
\hline $5,75 \mathrm{E}+07$ & $5,81 \mathrm{E}+07$ & $6,54 \mathrm{E}+07$ & $5,60 \mathrm{E}+07$ & $5,74 \mathrm{E}+07$ & $5,93 \mathrm{E}+07$ & $5,53 \mathrm{E}+07$ \\
\hline $5,80 \mathrm{E}+07$ & $5,83 \mathrm{E}+07$ & $6,59 \mathrm{E}+07$ & $5,70 \mathrm{E}+07$ & $5,78 \mathrm{E}+07$ & $5,97 \mathrm{E}+07$ & $5,66 \mathrm{E}+07$ \\
\hline $5,83 \mathrm{E}+07$ & $5,81 \mathrm{E}+07$ & $6,60 \mathrm{E}+07$ & $5,76 \mathrm{E}+07$ & $5,80 \mathrm{E}+07$ & $5,98 \mathrm{E}+07$ & $5,73 \mathrm{E}+07$ \\
\hline $5,84 \mathrm{E}+07$ & $5,80 \mathrm{E}+07$ & $6,60 \mathrm{E}+07$ & $5,80 \mathrm{E}+07$ & $5,83 \mathrm{E}+07$ & $5,98 \mathrm{E}+07$ & $5,77 \mathrm{E}+07$ \\
\hline $5,84 \mathrm{E}+07$ & $5,78 \mathrm{E}+07$ & $6,58 \mathrm{E}+07$ & $5,81 \mathrm{E}+07$ & $5,87 \mathrm{E}+07$ & $5,96 \mathrm{E}+07$ & $5,78 \mathrm{E}+07$ \\
\hline $5,85 \mathrm{E}+07$ & $5,77 \mathrm{E}+07$ & $6,56 \mathrm{E}+07$ & $5,82 \mathrm{E}+07$ & $5,90 \mathrm{E}+07$ & $5,95 \mathrm{E}+07$ & $5,78 \mathrm{E}+07$ \\
\hline $5,85 \mathrm{E}+07$ & $5,76 \mathrm{E}+07$ & $6,55 \mathrm{E}+07$ & $5,83 \mathrm{E}+07$ & $5,94 \mathrm{E}+07$ & $5,94 \mathrm{E}+07$ & $5,79 \mathrm{E}+07$ \\
\hline $5,87 \mathrm{E}+07$ & $5,77 \mathrm{E}+07$ & $6,55 \mathrm{E}+07$ & $5,84 \mathrm{E}+07$ & $5,99 \mathrm{E}+07$ & $5,94 \mathrm{E}+07$ & $5,79 \mathrm{E}+07$ \\
\hline $5,89 \mathrm{E}+07$ & $5,79 \mathrm{E}+07$ & $6,55 \mathrm{E}+07$ & $5,86 \mathrm{E}+07$ & $6,03 \mathrm{E}+07$ & & $5,79 \mathrm{E}+07$ \\
\hline $5,91 \mathrm{E}+07$ & & & $5,89 \mathrm{E}+07$ & $6,08 \mathrm{E}+07$ & & \\
\hline & & & $5,91 \mathrm{E}+07$ & & & \\
\hline & & & $5,94 \mathrm{E}+07$ & & & \\
\hline & & & $5,97 \mathrm{E}+07$ & & & \\
\hline & & $6,01 \mathrm{E}+07$ & & & \\
\hline & & & $\mathrm{C} 06$ & & \\
\hline
\end{tabular}

Continua 
Continuação do Anexo F

\begin{tabular}{|c|c|c|c|c|c|c|}
\hline 35 & 36 & 37 & 38 & 39 & 40 & 41 \\
\hline $1,51 \mathrm{E}+06$ & $1,72 \mathrm{E}+06$ & $1,60 \mathrm{E}+06$ & $1,43 \mathrm{E}+06$ & $1,48 \mathrm{E}+06$ & $1,73 \mathrm{E}+06$ & $1,56 \mathrm{E}+06$ \\
\hline $5,53 \mathrm{E}+06$ & $6,11 \mathrm{E}+06$ & $4,96 \mathrm{E}+06$ & $4,04 \mathrm{E}+06$ & $5,03 E+06$ & $5,57 \mathrm{E}+06$ & $4,39 \mathrm{E}+06$ \\
\hline $1,01 \mathrm{E}+07$ & $1,16 \mathrm{E}+$ & $9,05 \mathrm{E}+06$ & & $8,82 \mathrm{E}+06$ & & $3,20 \mathrm{E}+06$ \\
\hline $1,68 \mathrm{E}+07$ & $1,86 \mathrm{E}+07$ & $1,44 \mathrm{E}+07$ & $1,87 \mathrm{E}+07$ & & & $1,45 \mathrm{E}+07$ \\
\hline $2,37 \mathrm{E}+0$ & 2,5 & & & & & $2,21 \mathrm{E}+07$ \\
\hline $3,07 \mathrm{E}$ & 3,32 & & & & & +07 \\
\hline & & +07 & & & & +07 \\
\hline $4,38 \mathrm{E}+07$ & $4,59 \mathrm{E}+07$ & $3,80 \mathrm{E}+07$ & $4,17 \mathrm{E}+07$ & $3,75 \mathrm{E}$ & & $E+07$ \\
\hline $4,94 \mathrm{E}+07$ & $5,13 \mathrm{E}+07$ & $4,32 \mathrm{E}+07$ & & $4,11 \mathrm{E}$ & & $4,97 \mathrm{E}+07$ \\
\hline $5,34 \mathrm{E}$ & & $4,75 \mathrm{E}+07$ & & 4,41 & & $E+07$ \\
\hline $5,64 \mathrm{E}$ & $5,79 \mathrm{E}+07$ & $5,13 \mathrm{E}+07$ & $5,29 \mathrm{E}+07$ & $4,64 \mathrm{E}$ & & +07 \\
\hline $5,86 \mathrm{E}+07$ & $5,94 \mathrm{E}+07$ & $5,40 \mathrm{E}+07$ & $5,53 \mathrm{E}+07$ & $4,78 \mathrm{E}+07$ & $5,55 \mathrm{~b}+\mathrm{c}$ & $6,04 \mathrm{E}+07$ \\
\hline $5,97 \mathrm{E}+07$ & $6,01 \mathrm{E}+07$ & $5,63 \mathrm{E}+07$ & $5,45 \mathrm{E}+07$ & $4,85 \mathrm{E}+0 /$ & $3,0<E+U$ & $6,18 \mathrm{E}+07$ \\
\hline $6,01 \mathrm{E}$ & $6,05 \mathrm{E}+07$ & $5,77 \mathrm{E}+07$ & 5,80 & 4,90 & $5,8 c$ & $6,25 \mathrm{E}+07$ \\
\hline $6,02 \mathrm{E}$ & $6,07 \mathrm{E}+07$ & $5,88 \mathrm{E}+07$ & & 4,91 & & $6,27 \mathrm{E}+07$ \\
\hline & & 5,96 & & & & +07 \\
\hline $5,99 \mathrm{E}+07$ & $6,13 \mathrm{E}+07$ & $6,01 \mathrm{E}$ & $5,93 \mathrm{E}$ & 4,91 & 5,9 & $6,27 \mathrm{E}+07$ \\
\hline $5,97 \mathrm{E}+07$ & $6,19 \mathrm{E}+07$ & $6,04 \mathrm{E}+07$ & $5,96 \mathrm{E}+07$ & $4,91 \mathrm{E}+07$ & $5,91 \mathrm{E}+07$ & $6,28 \mathrm{E}+07$ \\
\hline $5,96 \mathrm{E}+07$ & $6,24 \mathrm{E}+07$ & $6,06 \mathrm{E}+07$ & $5,99 \mathrm{E}+07$ & $4,92 \mathrm{E}+07$ & $5,91 \mathrm{E}$ & $6,28 \mathrm{E}+07$ \\
\hline $5,96 \mathrm{E}+07$ & $6,30 \mathrm{E}+07$ & $6,09 \mathrm{E}+07$ & $6,02 \mathrm{E}+07$ & $4,92 \mathrm{E}+07$ & $5,90 \mathrm{E}+07$ & $6,30 \mathrm{E}+07$ \\
\hline $5,95 \mathrm{E}+07$ & $6,34 \mathrm{E}+07$ & $6,12 \mathrm{E}+07$ & 6,02 & 4,92 & 5,91 & $6,33 \mathrm{E}+07$ \\
\hline $5,96 \mathrm{E}+07$ & 6,3 & $6,1 !$ & $6,0 \varsigma$ & $4,9 ?$ & & $E+07$ \\
\hline & $6,41 \mathrm{E}$ & $6,18 \mathrm{E}$ & +07 & $4,94 \mathrm{E}+07$ & & $6,40 \mathrm{E}+07$ \\
\hline $5,99 \mathrm{E}+07$ & $6,44 \mathrm{E}+07$ & $6,21 \mathrm{E}+07$ & $6,15 \mathrm{E}+07$ & $4,95 \mathrm{E}+07$ & $5,95 \mathrm{E}$ & \\
\hline & $6,47 \mathrm{E}$ & 6,23 & & & & \\
\hline & $6,51 \mathrm{E}+07$ & $6,26 \mathrm{E}+07$ & $6,22 \mathrm{E}+07$ & & & \\
\hline
\end{tabular}

Continua 


\section{Continuação do Anexo F}

\begin{tabular}{|c|c|c|c|c|c|c|}
\hline 42 & 43 & 44 & 45 & 48 & 46 & Mediana \\
\hline $1,49 \mathrm{E}+06$ & $1,57 \mathrm{E}+06$ & $1,59 \mathrm{E}+06$ & $1,44 \mathrm{E}+06$ & $1,40 \mathrm{E}+06$ & $1,95 \mathrm{E}+06$ & 1569,03 \\
\hline $4,92 \mathrm{E}+06$ & $4,99 \mathrm{E}+06$ & $4,71 \mathrm{E}+06$ & $4,77 \mathrm{E}+06$ & $4,10 \mathrm{E}+06$ & $6,38 \mathrm{E}+06$ & 5303,57 \\
\hline $9,67 \mathrm{E}+06$ & $1,01 \mathrm{E}+07$ & $9,20 E+06$ & $1,51 \mathrm{E}+07$ & $7,37 \mathrm{E}+06$ & $1,15 \mathrm{E}+07$ & 10019,65 \\
\hline $1,61 \mathrm{E}+07$ & $1,68 \mathrm{E}+07$ & $1,44 \mathrm{E}+07$ & $2,21 \mathrm{E}+07$ & $1,20 \mathrm{E}+07$ & $1,78 \mathrm{E}+07$ & \\
\hline $2,31 \mathrm{E}+07$ & & & & & & \\
\hline $2,94 \mathrm{E}+07$ & 3,20 & & 3,42 & & & \\
\hline $3,56 \mathrm{E}+07$ & & & 4,00 & & & \\
\hline $4,08 \mathrm{E}+07$ & $4,41 \mathrm{E}$ & 3,78 & 4,42 & & +07 & 35,57 \\
\hline $4,52 \mathrm{E}+07$ & $4,92 \mathrm{E}+07$ & & & & $4,49 \mathrm{E}+07$ & 8,35 \\
\hline $4,89 \mathrm{E}+07$ & $5,34 \mathrm{E}$ & 4,71 & 5,14 & & & \\
\hline $5,14 \mathrm{E}+07$ & $5,61 \mathrm{E}+07$ & $5,01 \mathrm{E}$ & 5,321 & 4,38 & 5,0 & 3,72 \\
\hline $5,31 \mathrm{E}+07$ & $5,79 \mathrm{E}+07$ & $5,28 \mathrm{E}+07$ & $5,43 \mathrm{E}$ & $4,60 \mathrm{E}+07$ & $5,14 \mathrm{E}+07$ & 56343,04 \\
\hline $5,42 \mathrm{E}+07$ & $6,88 \mathrm{E}+0 /$ & $|b, 44 \mathrm{E}+0|$ & $5,52 \mathrm{E}$ & $4, / 4 \mathrm{t}$ & $5,22 \mathrm{E}+07$ & 57543,93 \\
\hline $5,47 \mathrm{E}+07$ & $5,92 \mathrm{E}$ & 5,54 & 5,54 & & +07 & 3,88 \\
\hline $5,50 \mathrm{E}$ & $5,94 t$ & 0,01 & 5,56 & 4,94 & +07 & 1,06 \\
\hline 5,52 & & & & & &, 76 \\
\hline $5,54 \mathrm{E}+07$ & $6,00 \mathrm{E}+07$ & $5,68 \mathrm{E}+07$ & $5,58 \mathrm{E}$ & $5,04 \mathrm{E}+07$ & $5,55 \mathrm{E}+07$ & 59400,30 \\
\hline $5,58 \mathrm{E}+07$ & $6,05 \mathrm{E}+07$ & $5,69 \mathrm{E}+07$ & $5,61 \mathrm{E}+07$ & $5,08 \mathrm{E}+07$ & $5,64 \mathrm{E}+07$ & 59525,54 \\
\hline $5,61 \mathrm{E}+07$ & $6,12 \mathrm{E}+07$ & $5,70 \mathrm{E}+07$ & $5,64 \mathrm{E}+07$ & $5,11 E+07$ & $5,75 \mathrm{E}+07$ & 59501,54 \\
\hline $5,66 \mathrm{E}$ & $6,20 \mathrm{E}$ & $5,71 \mathrm{E}$ & 5,69 & 5,12 & 5,85 & 59573,23 \\
\hline $5,71 \mathrm{E}$ & 6,27 & & & & 5,9 & \\
\hline & 6,34 & 5,74 & 5,7 & & 6,0 & \\
\hline $5,84 \mathrm{E}$ & $6,39 \mathrm{E}+07$ & $5,75 \mathrm{E}+07$ & $5,84 \mathrm{E}$ & $5,20 \mathrm{E}$ & 6,08 & \\
\hline $5,90 \mathrm{E}+07$ & $43 \mathrm{~F}+07$ & $5,76 \mathrm{E}+07$ & 5,90 & & & 59688,37 \\
\hline $5,96 \mathrm{E}+07$ & $6,46 \mathrm{E}+07$ & $5,78 \mathrm{E}+07$ & $5,96 \mathrm{E}+07$ & $5,26 \mathrm{E}+07$ & $6,20 \mathrm{E}+07$ & 60028,15 \\
\hline & & $5,80 E+07$ & $6,02 \mathrm{E}+0$ & 50050 & $6,24 \mathrm{E}+07$ & 7) 60815,77 \\
\hline
\end{tabular}


ANEXO G

DADOS DOS CORPOS DE PROVA DO GRUPO 2V 


\begin{tabular}{|c|c|c|c|c|c|c|}
\hline & $\mathbf{1}$ & $\mathbf{2}$ & $\mathbf{3}$ & $\mathbf{4}$ & $\mathbf{5}$ & $\mathbf{6}$ \\
\hline 0,00 & $1,54 \mathrm{E}+06$ & $1,50 \mathrm{E}+06$ & $1,48 \mathrm{E}+06$ & $1,70 \mathrm{E}+06$ & $1,52 \mathrm{E}+06$ & $1,49 \mathrm{E}+06$ \\
\hline 0,05 & $5,21 \mathrm{E}+06$ & $5,96 \mathrm{E}+06$ & $5,56 \mathrm{E}+06$ & $6,82 \mathrm{E}+06$ & $4,47 \mathrm{E}+06$ & $5,99 \mathrm{E}+06$ \\
\hline 0,10 & $1,03 \mathrm{E}+07$ & $1,11 \mathrm{E}+07$ & $1,07 \mathrm{E}+07$ & $1,27 \mathrm{E}+07$ & $8,00 \mathrm{E}+06$ & $1,16 \mathrm{E}+07$ \\
\hline 0,15 & $1,56 \mathrm{E}+07$ & $1,76 \mathrm{E}+07$ & $1,69 \mathrm{E}+07$ & $1,95 \mathrm{E}+07$ & $1,16 \mathrm{E}+07$ & $1,82 \mathrm{E}+07$ \\
\hline 0,20 & $2,18 \mathrm{E}+07$ & $2,35 \mathrm{E}+07$ & $2,29 \mathrm{E}+07$ & $2,48 \mathrm{E}+07$ & $1,61 \mathrm{E}+07$ & $2,56 \mathrm{E}+07$ \\
\hline 0,25 & $2,82 \mathrm{E}+07$ & $2,92 \mathrm{E}+07$ & $2,91 \mathrm{E}+07$ & $3,05 \mathrm{E}+07$ & $2,10 \mathrm{E}+07$ & $3,26 \mathrm{E}+07$ \\
\hline 0,30 & $3,47 \mathrm{E}+07$ & $3,44 \mathrm{E}+07$ & $3,54 \mathrm{E}+07$ & $3,57 \mathrm{E}+07$ & $2,57 \mathrm{E}+07$ & $3,94 \mathrm{E}+07$ \\
\hline 0,35 & $4,09 \mathrm{E}+07$ & $3,91 \mathrm{E}+07$ & $4,07 \mathrm{E}+07$ & $4,08 \mathrm{E}+07$ & $3,10 \mathrm{E}+07$ & $4,61 \mathrm{E}+07$ \\
\hline 0,40 & $4,57 \mathrm{E}+07$ & $4,31 \mathrm{E}+07$ & $4,52 \mathrm{E}+07$ & $4,53 \mathrm{E}+07$ & $3,58 \mathrm{E}+07$ & $5,08 \mathrm{E}+07$ \\
\hline 0,45 & $5,00 \mathrm{E}+07$ & $4,68 \mathrm{E}+07$ & $4,94 \mathrm{E}+07$ & $4,91 \mathrm{E}+07$ & $4,00 \mathrm{E}+07$ & $5,49 \mathrm{E}+07$ \\
\hline 0,50 & $5,36 \mathrm{E}+07$ & $5,00 \mathrm{E}+07$ & $5,32 \mathrm{E}+07$ & $5,20 \mathrm{E}+07$ & $4,34 \mathrm{E}+07$ & $5,86 \mathrm{E}+07$ \\
\hline 0,55 & $5,64 \mathrm{E}+07$ & $5,32 \mathrm{E}+07$ & $5,66 \mathrm{E}+07$ & $5,46 \mathrm{E}+07$ & $4,63 \mathrm{E}+07$ & $6,08 \mathrm{E}+07$ \\
\hline 0,60 & $5,90 \mathrm{E}+07$ & $5,57 \mathrm{E}+07$ & $5,91 \mathrm{E}+07$ & $5,64 \mathrm{E}+07$ & $4,90 \mathrm{E}+07$ & $6,28 \mathrm{E}+07$ \\
\hline 0,65 & $6,11 \mathrm{E}+07$ & $5,79 \mathrm{E}+07$ & $6,12 \mathrm{E}+07$ & $5,78 \mathrm{E}+07$ & $5,10 \mathrm{E}+07$ & $6,42 \mathrm{E}+07$ \\
\hline 0,70 & $6,28 \mathrm{E}+07$ & $5,97 \mathrm{E}+07$ & $6,27 \mathrm{E}+07$ & $5,89 \mathrm{E}+07$ & $5,30 \mathrm{E}+07$ & $6,50 \mathrm{E}+07$ \\
\hline 0,75 & $6,39 \mathrm{E}+07$ & $6,13 \mathrm{E}+07$ & $6,38 \mathrm{E}+07$ & $5,97 \mathrm{E}+07$ & $5,47 \mathrm{E}+07$ & $6,55 \mathrm{E}+07$ \\
\hline 0,80 & $6,49 \mathrm{E}+07$ & $6,26 \mathrm{E}+07$ & $6,46 \mathrm{E}+07$ & $6,03 \mathrm{E}+07$ & $5,62 \mathrm{E}+07$ & $6,57 \mathrm{E}+07$ \\
\hline 0,85 & $6,54 \mathrm{E}+07$ & $6,36 \mathrm{E}+07$ & $6,51 \mathrm{E}+07$ & $6,09 \mathrm{E}+07$ & $5,75 \mathrm{E}+07$ & $6,57 \mathrm{E}+07$ \\
\hline 0,90 & $6,28 \mathrm{E}+07$ & $6,45 \mathrm{E}+07$ & $6,55 \mathrm{E}+07$ & $6,14 \mathrm{E}+07$ & $5,88 \mathrm{E}+07$ & $6,57 \mathrm{E}+07$ \\
\hline 0,95 & $6,61 \mathrm{E}+07$ & $6,52 \mathrm{E}+07$ & $6,57 \mathrm{E}+07$ & $6,19 \mathrm{E}+07$ & $5,97 \mathrm{E}+07$ & $6,58 \mathrm{E}+07$ \\
\hline 1,00 & $6,62 \mathrm{E}+07$ & $6,57 \mathrm{E}+07$ & $6,58 \mathrm{E}+07$ & $6,24 \mathrm{E}+07$ & $6,05 \mathrm{E}+07$ & $6,58 \mathrm{E}+07$ \\
\hline 1,05 & $6,63 \mathrm{E}+07$ & $6,62 \mathrm{E}+07$ & $6,60 \mathrm{E}+07$ & $6,27 \mathrm{E}+07$ & $6,14 \mathrm{E}+07$ & $6,59 \mathrm{E}+07$ \\
\hline 1,10 & $6,65 \mathrm{E}+07$ & $6,65 \mathrm{E}+07$ & $6,61 \mathrm{E}+07$ & $6,32 \mathrm{E}+07$ & $6,21 \mathrm{E}+07$ & $6,60 \mathrm{E}+07$ \\
\hline 1,15 & & $6,68 \mathrm{E}+07$ & & $6,35 \mathrm{E}+07$ & $6,28 \mathrm{E}+07$ & \\
\hline 1,20 & & $6,71 \mathrm{E}+07$ & & $6,39 \mathrm{E}+07$ & $6,34 \mathrm{E}+07$ & \\
\hline 1,25 & & & & & & \\
\hline
\end{tabular}

Continua 
Continuação do Anexo G

\begin{tabular}{|c|c|c|c|c|c|c|}
\hline$r$ & 8 & 9 & 10 & 11 & 12 & \\
\hline $1,49 E+06$ & $1,53 E+06$ & $1,61 \mathrm{E}+06$ & $1,70 \mathrm{E}+06$ & $1,39 E+06$ & $1,41 E+06$ & $1,43 E+06$ \\
\hline $42 E+06$ & $4,83 E+06$ & $4,73 E+06$ & $6,83 E+06$ & & & $E+06$ \\
\hline $46 \mathrm{E}+06$ & & $8,17 \mathrm{E}+06$ & & & & \\
\hline ,40E+07 & $1,31 \mathrm{E}+07$ & $1,22 \mathrm{E}+07$ & $E+07$ & & & $1,59 \mathrm{E}+07$ \\
\hline $1,96 \mathrm{E}+07$ & $1,83 E+07$ & $1,73 \mathrm{E}+07$ & & & & $3 \mathrm{E}+07$ \\
\hline $2,52 \mathrm{E}+07$ & $2,35 \mathrm{E}+07$ & $2,30 \mathrm{E}+07$ & $E+07$ & 2,45 & & $2,82 E+07$ \\
\hline $3,00 E+07$ & & $2,84 \mathrm{E}+07$ & $E+07$ & & & $3,42 E+07$ \\
\hline $3,40 \mathrm{E}+07$ & $3,25 E+07$ & $3,29 \mathrm{E}+07$ & $4,26 \mathrm{E}+07$ & 3,59 & 2,9 & $3,94 \mathrm{E}+07$ \\
\hline $75 \mathrm{E}+07$ & & $3 \mathrm{E}+07$ & $E+07$ & & & $E+07$ \\
\hline $4,07 E+07$ & $9 \mathrm{E}+07$ & $4,29 E+07$ & $5,03 E+07$ & 4,50 & & $70 \mathrm{E}+07$ \\
\hline $35 \mathrm{E}+07$ & & $E+07$ & & & & +07 \\
\hline $4,58 \mathrm{E}+07$ & $5 \mathrm{E}+07$ & $5,07 \mathrm{E}+07$ & $E+07$ & $5,18 \mathrm{E}$ & & $5,27 \mathrm{E}+07$ \\
\hline $76 \mathrm{E}+07$ & $E+07$ & +07 & +07 & & & $5,49 \mathrm{E}+07$ \\
\hline $4,92 \mathrm{E}+07$ & $3 \mathrm{E}+07$ & $5,60 \mathrm{E}+07$ & $6 \mathrm{E}+07$ & $5,63 \mathrm{E}$ & & $5,67 \mathrm{E}+07$ \\
\hline, $05 E+07$ & $E+07$ & $E+07$ & +07 & 5,78 & & $5,77 \mathrm{E}+07$ \\
\hline $5,18 \mathrm{E}+07$ & $\mathrm{E}+07$ & $5,96 \mathrm{E}+07$ & $E+07$ & $5,91 \mathrm{E}_{+}$ & & $5,86 \mathrm{E}+07$ \\
\hline $5,29 \mathrm{E}+07$ & $E+07$ & $6,08 \mathrm{E}+07$ & +07 & 6,01 & & $3 E+07$ \\
\hline $5,38 \mathrm{E}+07$ & $0 \mathrm{E}+07$ & $6,19 \mathrm{E}+07$ & $5,93 E+07$ & $6,09 E_{+}$ & & $5,98 \mathrm{E}+07$ \\
\hline $5,47 \mathrm{E}+07$ & $6 \mathrm{E}+07$ & $6,30 \mathrm{E}+07$ & $5,96 \mathrm{E}+07$ & $6,14 \mathrm{E}_{+}$ & & $6,04 \mathrm{E}+07$ \\
\hline $5,55 \mathrm{E}+07$ & $2 E+07$ & $6,39 \mathrm{E}+07$ & $5,99 \mathrm{E}+07$ & $6,18 \mathrm{E}+$ & & $6,10 \mathrm{E}+07$ \\
\hline $5,61 \mathrm{E}+07$ & $67 \mathrm{E}+07$ & $6,47 \mathrm{E}+07$ & $6,01 E+07$ & $6,22 \mathrm{E}+$ & & $6,15 E+07$ \\
\hline $5,66 \mathrm{E}+07$ & $5,70 \mathrm{E}+07$ & $6,56 \mathrm{E}+07$ & $6,03 E+07$ & $6,25 E_{+}$ & & $6,20 \mathrm{E}+07$ \\
\hline $5,71 \mathrm{E}+07$ & $5,74 \mathrm{E}+07$ & $6,64 \mathrm{E}+07$ & $6,06 \mathrm{E}+07$ & $6,28 \mathrm{E}+07$ & & $6,25 E+07$ \\
\hline $5,75 E+07$ & $5,78 \mathrm{E}+07$ & $6,73 E+07$ & & $6,30 \mathrm{E}+$ & & $6,29 E+07$ \\
\hline $5,79 \mathrm{E}+07$ & $5,82 E+07$ & $6,80 \mathrm{E}+07$ & & $6,33 E+07$ & $5,46 E+0$ & $6,34 \mathrm{E}+07$ \\
\hline $5,83 E+07$ & & & & & $5,53 E+07$ & \\
\hline
\end{tabular}

Continua 
Continuação do Anexo G

\begin{tabular}{|c|c|c|c|c|c|c|}
\hline 14 & 15 & 16 & 17 & 18 & 19 & 20 \\
\hline $1,53 \mathrm{E}+06$ & $1,56 \mathrm{E}+06$ & $1,52 \mathrm{E}+0 \mathrm{G}$ & $1,29 \mathrm{E}+06$ & $1,56 \mathrm{E}+06$ & $1,61 \mathrm{E}+06$ & $1,47 E+06$ \\
\hline $6,14 \mathrm{E}+06$ & $6,65 \mathrm{E}+06$ & $4,57 \mathrm{E}+0 \mathrm{f}$ & $3,85 \mathrm{E}+06$ & $5,69 \mathrm{E}+0$ & $4,14 \mathrm{E}+\mathrm{C}$ & $4,53 E+06$ \\
\hline $1,02 \mathrm{E}+07$ & $1,18 \mathrm{E}+07$ & $8,40 \mathrm{E}+0 \mathrm{E}$ & $6,52 \mathrm{E}+06$ & $1,02 \mathrm{E}+07$ & 7,34 & $9,57 \mathrm{E}+06$ \\
\hline $1,56 \mathrm{E}+07$ & $1,75 \mathrm{E}+07$ & $1,38 \mathrm{E}+07$ & $9,73 \mathrm{E}+06$ & $1,49 \mathrm{E}+07$ & $1,07 \mathrm{E}+07$ & $1,55 \mathrm{E}+07$ \\
\hline $2,17 \mathrm{E}+07$ & $2,27 \mathrm{E}+07$ & $2,00 \mathrm{E}+07$ & $1,35 \mathrm{E}+07$ & $1,99 \mathrm{E}+07$ & +0 & $2,16 \mathrm{E}+07$ \\
\hline $2,76 \mathrm{E}+07$ & $2,97 \mathrm{E}+07$ & $2,56 \mathrm{E}+07$ & $1,73 \mathrm{E}+0$ & $2,52 \mathrm{E}+$ & & $2,68 \mathrm{E}+07$ \\
\hline $3,32 \mathrm{E}+07$ & $3,48 \mathrm{E}+07$ & $3,12 \mathrm{E}+07$ & $2,14 \mathrm{E}+\mathrm{C}$ & 2065 & & $3,15 \mathrm{E}+07$ \\
\hline $3,88 \mathrm{E}+07$ & $3,96 \mathrm{E}+07$ & $3,71 \mathrm{E}+0$ & $2,53 \mathrm{E}+\mathrm{C}$ & $3,38 \mathrm{E}+$ & & $3,56 \mathrm{E}+07$ \\
\hline $4,35 \mathrm{E}+07$ & $4,39 \mathrm{E}+07$ & $4,15 \mathrm{E}+07$ & $2,94 \mathrm{E}+07$ & $3,78 \mathrm{E}+07$ & $E+07$ & $3,88 \mathrm{E}+07$ \\
\hline $4,75 \mathrm{E}+07$ & $4,80 \mathrm{E}+07$ & $4,58 \mathrm{E}+07$ & $3,29 \mathrm{E}+07$ & $4,09 \mathrm{E}+07$ & $3,70 \mathrm{E}+0$ & $4,17 \mathrm{E}+07$ \\
\hline $5,11 \mathrm{E}+07$ & $5,13 \mathrm{E}+07$ & $4,91 \mathrm{E}+07$ & $3,70 \mathrm{E}+07$ & $4,37 \mathrm{E}+07$ & 4,1 & $4,39 E+07$ \\
\hline $5,43 \mathrm{E}+07$ & $5,38 \mathrm{E}+07$ & $5,20 \mathrm{E}+07$ & $4,04 \mathrm{E}+07$ & $4,60 \mathrm{E}+07$ & $4,51 \mathrm{E}+0$ & $4,55 \mathrm{E}+07$ \\
\hline $5,70 \mathrm{E}+07$ & $5,60 \mathrm{E}+07$ & $5,43 \mathrm{E}+07$ & $4,35 \mathrm{E}+07$ & $4,83 \mathrm{E}+07$ & $4,87 \mathrm{E}+\mathrm{C}$ & $4,65 \mathrm{E}+07$ \\
\hline $5,95 \mathrm{E}+07$ & $5,75 \mathrm{E}+07$ & $5,61 \mathrm{E}+07$ & $4,61 \mathrm{E}+07$ & $5,02 \mathrm{E}+0$ & & $4,72 \mathrm{E}+07$ \\
\hline $6,14 \mathrm{E}+07$ & $5,87 \mathrm{E}+07$ & $5,76 \mathrm{E}+07$ & $4,86 \mathrm{E}+07$ & $5,20 \mathrm{E}+07$ & 5,45 & $4,79 \mathrm{E}+07$ \\
\hline $6,29 \mathrm{E}+07$ & $5,97 \mathrm{E}+07$ & $5,89 \mathrm{E}+07$ & $5,08 \mathrm{E}+07$ & $5,37 \mathrm{E}+$ & & $4,86 \mathrm{E}+07$ \\
\hline $6,40 \mathrm{E}+07$ & $6,03 \mathrm{E}+07$ & $5,98 \mathrm{E}+07$ & $5,26 \mathrm{E}+07$ & $5,53 \mathrm{E}+07$ & 5,79 & $4,96 \mathrm{E}+07$ \\
\hline $6,47 \mathrm{E}+07$ & $6,10 \mathrm{E}+07$ & $6,06 \mathrm{E}+07$ & $5,41 \mathrm{E}+07$ & $5,66 \mathrm{E}+07$ & $5,89 \mathrm{E}+07$ & $5,07 \mathrm{E}+07$ \\
\hline $6,53 \mathrm{E}+07$ & $6,16 \mathrm{E}+07$ & $6,14 \mathrm{E}+07$ & $5,53 \mathrm{E}+07$ & $5,78 \mathrm{E}+07$ & $5,95 \mathrm{E}+0$ & $5,18 \mathrm{E}+07$ \\
\hline $6,57 \mathrm{E}+07$ & $6,20 \mathrm{E}+07$ & $6,21 \mathrm{E}+07$ & $5,60 \mathrm{E}+07$ & $5,90 \mathrm{E}+07$ & $5,98 \mathrm{E}+0$ & $5,29 E+07$ \\
\hline $6,60 \mathrm{E}+07$ & $6,26 \mathrm{E}+07$ & $6,26 \mathrm{E}+07$ & $5,65 \mathrm{E}+07$ & $6,01 \mathrm{E}+07$ & 6,0 & $5,38 \mathrm{E}+07$ \\
\hline $6,62 \mathrm{E}+07$ & $6,29 \mathrm{E}+07$ & $6,31 \mathrm{E}+07$ & $5,68 \mathrm{E}+07$ & $6,11 \mathrm{E}+0$ & & $5,47 \mathrm{E}+07$ \\
\hline $6,64 \mathrm{E}+07$ & $6,32 \mathrm{E}+07$ & $6,34 \mathrm{E}+07$ & $5,70 \mathrm{E}+07$ & $6,21 \mathrm{E}+07$ & $6,02 \mathrm{E}+07$ & $5,54 \mathrm{E}+07$ \\
\hline & & $6,39 \mathrm{E}+07$ & $5,74 \mathrm{E}+07$ & $6,31 \mathrm{E}+$ & & $5,60 \mathrm{E}+07$ \\
\hline & & $6,41 \mathrm{E}+07$ & $5,76 \mathrm{E}+07$ & $6,40 \mathrm{E}+07$ & $6,05 \mathrm{E}+07$ & $5,65 \mathrm{E}+07$ \\
\hline & & & $5,79 \mathrm{E}+07$ & $6,50 \mathrm{E}+07$ & & $5,70 \mathrm{E}+07$ \\
\hline
\end{tabular}

Continua 
Continuação do Anexo G

\begin{tabular}{|c|c|c|c|c|c|c|}
\hline $\mathbf{2 1}$ & $\mathbf{2 3}$ & $\mathbf{2 4}$ & $\mathbf{2 5}$ & $\mathbf{2 6}$ & $\mathbf{2 7}$ & $\mathbf{2 8}$ \\
\hline $1,48 \mathrm{E}+06$ & $1,40 \mathrm{E}+06$ & $1,38 \mathrm{E}+06$ & $1,46 \mathrm{E}+06$ & $1,39 \mathrm{E}+06$ & $1,45 \mathrm{E}+06$ & $1,65 \mathrm{E}+06$ \\
\hline $6,26 \mathrm{E}+06$ & $5,44 \mathrm{E}+06$ & $5,79 \mathrm{E}+06$ & $5,97 \mathrm{E}+06$ & $4,31 \mathrm{E}+06$ & $5,34 \mathrm{E}+06$ & $6,08 \mathrm{E}+06$ \\
\hline $1,19 \mathrm{E}+07$ & $9,16 \mathrm{E}+06$ & $1,17 \mathrm{E}+07$ & $1,02 \mathrm{E}+07$ & $7,81 \mathrm{E}+06$ & $8,74 \mathrm{E}+06$ & $1,14 \mathrm{E}+07$ \\
\hline $1,85 \mathrm{E}+07$ & $1,46 \mathrm{E}+07$ & $1,87 \mathrm{E}+07$ & $1,61 \mathrm{E}+07$ & $1,27 \mathrm{E}+07$ & $1,44 \mathrm{E}+07$ & $1,82 \mathrm{E}+07$ \\
\hline $2,58 \mathrm{E}+07$ & $2,05 \mathrm{E}+07$ & $2,50 \mathrm{E}+07$ & $2,25 \mathrm{E}+07$ & $1,85 \mathrm{E}+07$ & $1,96 \mathrm{E}+07$ & $2,50 \mathrm{E}+07$ \\
\hline $3,22 \mathrm{E}+07$ & $2,52 \mathrm{E}+07$ & $3,15 \mathrm{E}+07$ & $2,85 \mathrm{E}+07$ & $2,40 \mathrm{E}+07$ & $2,56 \mathrm{E}+07$ & $3,20 \mathrm{E}+07$ \\
\hline $3,77 \mathrm{E}+07$ & $3,06 \mathrm{E}+07$ & $3,76 \mathrm{E}+07$ & $3,40 \mathrm{E}+07$ & $3,01 \mathrm{E}+07$ & $3,10 \mathrm{E}+07$ & $3,90 \mathrm{E}+07$ \\
\hline $4,34 \mathrm{E}+07$ & $3,61 \mathrm{E}+07$ & $4,28 \mathrm{E}+07$ & $3,95 \mathrm{E}+07$ & $3,47 \mathrm{E}+07$ & $3,68 \mathrm{E}+07$ & $4,48 \mathrm{E}+07$ \\
\hline $4,76 \mathrm{E}+07$ & $4,17 \mathrm{E}+07$ & $4,74 \mathrm{E}+07$ & $4,36 \mathrm{E}+07$ & $3,94 \mathrm{E}+07$ & $4,19 \mathrm{E}+07$ & $5,02 \mathrm{E}+07$ \\
\hline $5,13 \mathrm{E}+07$ & $4,66 \mathrm{E}+07$ & $5,10 \mathrm{E}+07$ & $4,70 \mathrm{E}+07$ & $4,40 \mathrm{E}+07$ & $4,66 \mathrm{E}+07$ & $5,44 \mathrm{E}+07$ \\
\hline $5,48 \mathrm{E}+07$ & $5,08 \mathrm{E}+07$ & $5,39 \mathrm{E}+07$ & $4,97 \mathrm{E}+07$ & $4,81 \mathrm{E}+07$ & $5,10 \mathrm{E}+07$ & $5,82 \mathrm{E}+07$ \\
\hline $5,74 \mathrm{E}+07$ & $5,53 \mathrm{E}+07$ & $5,60 \mathrm{E}+07$ & $5,21 \mathrm{E}+07$ & $5,22 \mathrm{E}+07$ & $5,45 \mathrm{E}+07$ & $6,08 \mathrm{E}+07$ \\
\hline $5,94 \mathrm{E}+07$ & $5,89 \mathrm{E}+07$ & $5,76 \mathrm{E}+07$ & $5,38 \mathrm{E}+07$ & $5,56 \mathrm{E}+07$ & $5,73 \mathrm{E}+07$ & $6,26 \mathrm{E}+07$ \\
\hline $6,08 \mathrm{E}+07$ & $6,20 \mathrm{E}+07$ & $5,86 \mathrm{E}+07$ & $5,51 \mathrm{E}+07$ & $5,87 \mathrm{E}+07$ & $5,95 \mathrm{E}+07$ & $6,40 \mathrm{E}+07$ \\
\hline $6,22 \mathrm{E}+07$ & $6,43 \mathrm{E}+07$ & $5,90 \mathrm{E}+07$ & $5,69 \mathrm{E}+07$ & $6,07 \mathrm{E}+07$ & $6,12 \mathrm{E}+07$ & $6,49 \mathrm{E}+07$ \\
\hline $6,33 \mathrm{E}+07$ & $6,60 \mathrm{E}+07$ & $5,93 \mathrm{E}+07$ & $5,74 \mathrm{E}+07$ & $6,22 \mathrm{E}+07$ & $6,24 \mathrm{E}+07$ & $6,54 \mathrm{E}+07$ \\
\hline $6,40 \mathrm{E}+07$ & $6,70 \mathrm{E}+07$ & $5,95 \mathrm{E}+07$ & $5,79 \mathrm{E}+07$ & $6,32 \mathrm{E}+07$ & $6,31 \mathrm{E}+07$ & $6,56 \mathrm{E}+07$ \\
\hline $6,45 \mathrm{E}+07$ & $6,75 \mathrm{E}+07$ & $5,96 \mathrm{E}+07$ & $5,83 \mathrm{E}+07$ & $6,37 \mathrm{E}+07$ & $6,36 \mathrm{E}+07$ & $6,56 \mathrm{E}+07$ \\
\hline $6,50 \mathrm{E}+07$ & $6,78 \mathrm{E}+07$ & $5,98 \mathrm{E}+07$ & $5,86 \mathrm{E}+07$ & $6,41 \mathrm{E}+07$ & $6,38 \mathrm{E}+07$ & $6,56 \mathrm{E}+07$ \\
\hline $6,56 \mathrm{E}+07$ & $6,79 \mathrm{E}+07$ & $5,99 \mathrm{E}+07$ & $5,89 \mathrm{E}+07$ & $6,44 \mathrm{E}+07$ & $6,39 \mathrm{E}+07$ & $6,56 \mathrm{E}+07$ \\
\hline $6,59 \mathrm{E}+07$ & $6,78 \mathrm{E}+07$ & $6,01 \mathrm{E}+07$ & $5,93 \mathrm{E}+07$ & $6,45 \mathrm{E}+07$ & $6,40 \mathrm{E}+07$ & $6,56 \mathrm{E}+07$ \\
\hline $6,64 \mathrm{E}+07$ & $6,78 \mathrm{E}+07$ & $6,02 \mathrm{E}+07$ & $5,97 \mathrm{E}+07$ & $6,46 \mathrm{E}+07$ & $6,40 \mathrm{E}+07$ & $6,56 \mathrm{E}+07$ \\
\hline $6,69 \mathrm{E}+07$ & $6,78 \mathrm{E}+07$ & $6,05 \mathrm{E}+07$ & $6,00 \mathrm{E}+07$ & $6,46 \mathrm{E}+07$ & $6,41 \mathrm{E}+07$ & $6,56 \mathrm{E}+07$ \\
\hline & & & $6,03 \mathrm{E}+07$ & & $6,41 \mathrm{E}+07$ & \\
\hline & & & $6,07 \mathrm{E}+07$ & & $6,43 \mathrm{E}+07$ & \\
\hline & & & $6,11 \mathrm{E}+07$ & & & \\
\hline
\end{tabular}

Continua 


\section{Continuação do Anexo G}

\begin{tabular}{|c|c|c|c|c|c|c|}
\hline 29 & 30 & 31 & 32 & 33 & 34 & 35 \\
\hline $1,23 E+06$ & $1,43 E+06$ & $1,50 \mathrm{E}+06$ & $1,46 \mathrm{E}+06$ & $1,43 \mathrm{E}+06$ & $1,36 \mathrm{E}+06$ & $1,52 E+06$ \\
\hline $5,02 E+06$ & $3,70 E+06$ & $4,92 E+06$ & $4,53 \mathrm{E}+06$ & $5,16 \mathrm{E}+06$ & $3,41 \mathrm{E}+06$ & $4,65 \mathrm{E}+06$ \\
\hline $1,14 \mathrm{E}+07$ & & & $8,41 \mathrm{E}+06$ & & $655 F+06$ & $7,90 \mathrm{E}+06$ \\
\hline $1,57 \mathrm{E}+07$ & $1,03 E+07$ & $1,50 \mathrm{E}+07$ & $1,33 \mathrm{E}+07$ & $1,60 \mathrm{E}+07$ & $1,06 \mathrm{E}+07$ & ,21E+07 \\
\hline $2,19 \mathrm{E}+07$ & & & & & & $1,63 E+07$ \\
\hline $2,93 E+07$ & $2,02 E+07$ & $2,95 \mathrm{E}+07$ & $2,45 \mathrm{E}$ & $2,85 \mathrm{E}+07$ & $2,14 \mathrm{E}+07$ & $2,06 E+07$ \\
\hline $3,45 \mathrm{E}+07$ & & & $2,98 \mathrm{E}$ & & & $E+07$ \\
\hline $4,04 \mathrm{E}+07$ & $3,28 E+07$ & 4,25 & $3,49 \mathrm{E}+07$ & $4,03 \mathrm{E}+07$ & $3,20 \mathrm{E}+07$ & $3,07 E+07$ \\
\hline $4,57 \mathrm{E}+07$ & & & & & & $E+07$ \\
\hline $4,97 \mathrm{E}+07$ & $4,37 \mathrm{E}$ & 5,33 & $4,26 \mathrm{E}+07$ & $5,05 \mathrm{E}+07$ & $4,22 E+07$ & $4,03 E+07$ \\
\hline $5,30 \mathrm{E}+07$ & & & $4,59 \mathrm{E}+07$ & & & $E+07$ \\
\hline $5,56 \mathrm{E}+07$ & $5,11 E+07$ & $6,03 \mathrm{E}$ & $4,88 \mathrm{E}+07$ & $5,73 \mathrm{E}+07$ & $4,86 \mathrm{E}+07$ & $4,83 E+07$ \\
\hline $5,76 \mathrm{E}+07$ & & & +07 & 5,9 & -07 & $E+07$ \\
\hline $5,88 \mathrm{E}+07$ & $5,59 \mathrm{E}+07$ & $6,38 \mathrm{~B}$ & $5,37 \mathrm{E}+07$ & $\mathrm{E}+07$ & $5,25 \mathrm{E}+07$ & $5,45 \mathrm{E}+07$ \\
\hline $5,96 \mathrm{E}+07$ & $5,73 \mathrm{E}+07$ & 6,47 & $5,57 \mathrm{E}+07$ & $E+07$ & +07 & $E+07$ \\
\hline $6,00 \mathrm{E}+07$ & $5,82 \mathrm{E}+07$ & $6,52 \mathrm{E}$ & $5,74 \mathrm{E}+07$ & $0 \mathrm{E}+07$ & $5,45 \mathrm{E}+07$ & $5,85 E+07$ \\
\hline $6,01 \mathrm{E}+07$ & $5,88 \mathrm{E}+07$ & $6,53 \mathrm{E}$ & $5,87 \mathrm{E}+07$ & $6,32 \mathrm{E}+07$ & $5,50 \mathrm{E}+07$ & $5,99 \mathrm{E}+07$ \\
\hline $6,00 \mathrm{E}+07$ & $5,94 \mathrm{E}+07$ & $6,54 \mathrm{E}$ & $5,97 \mathrm{E}+07$ & $6,33 \mathrm{E}+07$ & $5,53 \mathrm{E}+07$ & $6,10 \mathrm{E}+07$ \\
\hline $5,98 \mathrm{E}+07$ & $5,98 \mathrm{E}+07$ & $6,54 \mathrm{E}+07$ & $6,06 \mathrm{E}+07$ & $6,33 \mathrm{E}+07$ & $5,55 E+07$ & $6,19 \mathrm{E}+07$ \\
\hline $5,97 \mathrm{E}+07$ & $6,01 \mathrm{E}+07$ & $6,53 \mathrm{E}+07$ & $6,12 \mathrm{E}+07$ & $6,34 \mathrm{E}+07$ & $5,58 \mathrm{E}+07$ & $6,27 E+07$ \\
\hline $5,97 E+07$ & $6,04 \mathrm{E}+07$ & $6,53 \mathrm{E}+07$ & $6,18 \mathrm{E}+07$ & $6,34 \mathrm{E}+07$ & $5,60 \mathrm{E}+07$ & $6,32 E+07$ \\
\hline $5,96 \mathrm{E}+07$ & $6,06 \mathrm{E}+07$ & $6,54 \mathrm{E}+07$ & $6,22 E+07$ & $6,34 \mathrm{E}+07$ & $5,62 \mathrm{E}+07$ & $6,35 \mathrm{E}+07$ \\
\hline \multirow[t]{4}{*}{$5,96 \mathrm{E}+07$} & $6,09 \mathrm{E}+07$ & $6,55 \mathrm{E}+07$ & $6,28 \mathrm{E}+07$ & $6,36 \mathrm{E}+07$ & $5,64 \mathrm{E}+07$ & $6,39 E+07$ \\
\hline & & & & & & $6,41 \mathrm{E}+07$ \\
\hline & & & & & & $6,43 E+07$ \\
\hline & & & & & & \\
\hline
\end{tabular}

Continua 
Continuação do Anexo G

\begin{tabular}{|c|c|c|c|c|c|c|}
\hline $\mathbf{3 6}$ & $\mathbf{3 7}$ & $\mathbf{3 8}$ & $\mathbf{3 9}$ & $\mathbf{4 0}$ & $\mathbf{4 1}$ & $\mathbf{4 2}$ \\
\hline $1,37 \mathrm{E}+06$ & $1,40 \mathrm{E}+06$ & $1,48 \mathrm{E}+06$ & $1,49 \mathrm{E}+06$ & $1,45 \mathrm{E}+06$ & $1,49 \mathrm{E}+06$ & $1,49 \mathrm{E}+06$ \\
\hline $4,53 \mathrm{E}+06$ & $5,25 \mathrm{E}+06$ & $4,23 \mathrm{E}+06$ & $6,04 \mathrm{E}+06$ & $7,02 \mathrm{E}+06$ & $6,02 \mathrm{E}+06$ & $7,27 \mathrm{E}+06$ \\
\hline $8,19 \mathrm{E}+06$ & $1,06 \mathrm{E}+07$ & $6,81 \mathrm{E}+06$ & $1,27 \mathrm{E}+07$ & $1,26 \mathrm{E}+07$ & $1,09 \mathrm{E}+07$ & $1,31 \mathrm{E}+07$ \\
\hline $1,32 \mathrm{E}+07$ & $1,64 \mathrm{E}+07$ & $1,01 \mathrm{E}+07$ & $2,08 \mathrm{E}+07$ & $1,89 \mathrm{E}+07$ & $1,62 \mathrm{E}+07$ & $1,97 \mathrm{E}+07$ \\
\hline $1,85 \mathrm{E}+07$ & $2,33 \mathrm{E}+07$ & $1,36 \mathrm{E}+07$ & $2,85 \mathrm{E}+07$ & $2,44 \mathrm{E}+07$ & $2,16 \mathrm{E}+07$ & $2,58 \mathrm{E}+07$ \\
\hline $2,43 \mathrm{E}+07$ & $3,04 \mathrm{E}+07$ & $1,75 \mathrm{E}+07$ & $3,61 \mathrm{E}+07$ & $3,00 \mathrm{E}+07$ & $2,70 \mathrm{E}+07$ & $3,18 \mathrm{E}+07$ \\
\hline $2,97 \mathrm{E}+07$ & $3,67 \mathrm{E}+07$ & $2,14 \mathrm{E}+07$ & $4,25 \mathrm{E}+07$ & $3,55 \mathrm{E}+07$ & $3,16 \mathrm{E}+07$ & $3,68 \mathrm{E}+07$ \\
\hline $3,55 \mathrm{E}+07$ & $4,17 \mathrm{E}+07$ & $2,52 \mathrm{E}+07$ & $4,81 \mathrm{E}+07$ & $4,07 \mathrm{E}+07$ & $3,65 \mathrm{E}+07$ & $4,19 \mathrm{E}+07$ \\
\hline $3,98 \mathrm{E}+07$ & $4,62 \mathrm{E}+07$ & $2,86 \mathrm{E}+07$ & $5,32 \mathrm{E}+07$ & $4,58 \mathrm{E}+07$ & $4,08 \mathrm{E}+07$ & $4,61 \mathrm{E}+07$ \\
\hline $4,39 \mathrm{E}+07$ & $5,01 \mathrm{E}+07$ & $3,16 \mathrm{E}+07$ & $5,74 \mathrm{E}+07$ & $5,03 \mathrm{E}+07$ & $4,54 \mathrm{E}+07$ & $5,01 \mathrm{E}+07$ \\
\hline $4,75 \mathrm{E}+07$ & $5,31 \mathrm{E}+07$ & $3,41 \mathrm{E}+07$ & $6,14 \mathrm{E}+07$ & $5,40 \mathrm{E}+07$ & $4,92 \mathrm{E}+07$ & $5,31 \mathrm{E}+07$ \\
\hline $5,07 \mathrm{E}+07$ & $5,53 \mathrm{E}+07$ & $3,61 \mathrm{E}+07$ & $6,42 \mathrm{E}+07$ & $5,72 \mathrm{E}+07$ & $5,28 \mathrm{E}+07$ & $5,56 \mathrm{E}+07$ \\
\hline $5,38 \mathrm{E}+07$ & $5,70 \mathrm{E}+07$ & $3,80 \mathrm{E}+07$ & $6,61 \mathrm{E}+07$ & $5,98 \mathrm{E}+07$ & $5,55 \mathrm{E}+07$ & $5,75 \mathrm{E}+07$ \\
\hline $5,63 \mathrm{E}+07$ & $5,81 \mathrm{E}+07$ & $3,96 \mathrm{E}+07$ & $6,71 \mathrm{E}+07$ & $6,16 \mathrm{E}+07$ & $5,75 \mathrm{E}+07$ & $5,91 \mathrm{E}+07$ \\
\hline $5,80 \mathrm{E}+07$ & $5,88 \mathrm{E}+07$ & $4,11 \mathrm{E}+07$ & $6,76 \mathrm{E}+07$ & $6,25 \mathrm{E}+07$ & $5,86 \mathrm{E}+07$ & $6,03 \mathrm{E}+07$ \\
\hline $5,93 \mathrm{E}+07$ & $5,91 \mathrm{E}+07$ & $4,25 \mathrm{E}+07$ & $6,77 \mathrm{E}+07$ & $6,30 \mathrm{E}+07$ & $5,92 \mathrm{E}+07$ & $6,12 \mathrm{E}+07$ \\
\hline $6,03 \mathrm{E}+07$ & $5,92 \mathrm{E}+07$ & $4,39 \mathrm{E}+07$ & $6,76 \mathrm{E}+07$ & $6,30 \mathrm{E}+07$ & $5,95 \mathrm{E}+07$ & $6,20 \mathrm{E}+07$ \\
\hline $6,10 \mathrm{E}+07$ & $5,93 \mathrm{E}+07$ & $4,53 \mathrm{E}+07$ & $6,75 \mathrm{E}+07$ & $6,32 \mathrm{E}+07$ & $5,97 \mathrm{E}+07$ & $6,27 \mathrm{E}+07$ \\
\hline $6,14 \mathrm{E}+07$ & $5,93 \mathrm{E}+07$ & $4,64 \mathrm{E}+07$ & $6,74 \mathrm{E}+07$ & $6,25 \mathrm{E}+07$ & $5,99 \mathrm{E}+07$ & $6,33 \mathrm{E}+07$ \\
\hline $6,18 \mathrm{E}+07$ & $5,93 \mathrm{E}+07$ & $4,77 \mathrm{E}+07$ & $6,73 \mathrm{E}+07$ & $6,36 \mathrm{E}+07$ & $6,04 \mathrm{E}+07$ & $6,39 \mathrm{E}+07$ \\
\hline $6,21 \mathrm{E}+07$ & $5,93 \mathrm{E}+07$ & $4,89 \mathrm{E}+07$ & $6,71 \mathrm{E}+07$ & $6,40 \mathrm{E}+07$ & $6,09 \mathrm{E}+07$ & $6,44 \mathrm{E}+07$ \\
\hline $6,23 \mathrm{E}+07$ & $5,94 \mathrm{E}+07$ & $5,00 \mathrm{E}+07$ & $6,72 \mathrm{E}+07$ & $6,45 \mathrm{E}+07$ & $6,14 \mathrm{E}+07$ & $6,49 \mathrm{E}+07$ \\
\hline $6,25 \mathrm{E}+07$ & $5,96 \mathrm{E}+07$ & $5,12 \mathrm{E}+07$ & $6,74 \mathrm{E}+07$ & $6,49 \mathrm{E}+07$ & $6,19 \mathrm{E}+07$ & $6,53 \mathrm{E}+07$ \\
\hline & & $5,23 \mathrm{E}+07$ & & & $6,25 \mathrm{E}+07$ & \\
\hline & $5,34 \mathrm{E}+07$ & & & $6,31 \mathrm{E}+07$ & \\
\hline & $5,46 \mathrm{E}+07$ & & & $6,35 \mathrm{E}+07$ & \\
\hline
\end{tabular}

Continua 


\section{Continuação do Anexo G}

\begin{tabular}{|c|c|c|c|c|c|c|}
\hline $\mathbf{4 3}$ & $\mathbf{4 4}$ & $\mathbf{4 5}$ & $\mathbf{4 6}$ & $\mathbf{4 7}$ & $\mathbf{4 8}$ & Mediana \\
\hline $1,28 \mathrm{E}+06$ & $1,43 \mathrm{E}+06$ & $1,35 \mathrm{E}+06$ & $1,41 \mathrm{E}+06$ & $1,55 \mathrm{E}+06$ & $1,54 \mathrm{E}+06$ & 1481,02 \\
\hline $4,93 \mathrm{E}+06$ & $6,34 \mathrm{E}+06$ & $4,35 \mathrm{E}+06$ & $3,51 \mathrm{E}+06$ & $4,25 \mathrm{E}+06$ & $6,19 \mathrm{E}+06$ & 5092,71 \\
\hline $9,42 \mathrm{E}+06$ & $1,18 \mathrm{E}+07$ & $9,48 \mathrm{E}+06$ & $5,69 \mathrm{E}+06$ & $8,11 \mathrm{E}+06$ & $1,32 \mathrm{E}+07$ & 9603,54 \\
\hline $1,52 \mathrm{E}+07$ & $1,88 \mathrm{E}+07$ & $1,58 \mathrm{E}+07$ & $8,28 \mathrm{E}+06$ & $1,27 \mathrm{E}+07$ & $1,95 \mathrm{E}+07$ & 15360,13 \\
\hline $2,15 \mathrm{E}+07$ & $2,55 \mathrm{E}+07$ & $2,23 \mathrm{E}+07$ & $1,14 \mathrm{E}+07$ & $1,77 \mathrm{E}+07$ & $2,64 \mathrm{E}+07$ & 21593,49 \\
\hline $2,78 \mathrm{E}+07$ & $3,22 \mathrm{E}+07$ & $2,91 \mathrm{E}+07$ & $1,51 \mathrm{E}+07$ & $2,31 \mathrm{E}+07$ & $3,32 \mathrm{E}+07$ & 27306,18 \\
\hline $3,40 \mathrm{E}+07$ & $3,84 \mathrm{E}+07$ & $3,57 \mathrm{E}+07$ & $1,95 \mathrm{E}+07$ & $2,85 \mathrm{E}+07$ & $3,96 \mathrm{E}+07$ & 32388,36 \\
\hline $4,05 \mathrm{E}+07$ & $4,39 \mathrm{E}+07$ & $4,16 \mathrm{E}+07$ & $2,40 \mathrm{E}+07$ & $3,36 \mathrm{E}+07$ & $4,53 \mathrm{E}+07$ & 37928,03 \\
\hline $4,62 \mathrm{E}+07$ & $4,86 \mathrm{E}+07$ & $4,67 \mathrm{E}+07$ & $2,88 \mathrm{E}+07$ & $3,85 \mathrm{E}+07$ & $5,03 \mathrm{E}+07$ & 42484,36 \\
\hline $5,08 \mathrm{E}+07$ & $5,22 \mathrm{E}+07$ & $5,04 \mathrm{E}+07$ & $3,33 \mathrm{E}+07$ & $4,30 \mathrm{E}+07$ & $5,49 \mathrm{E}+07$ & 46747,00 \\
\hline $5,50 \mathrm{E}+07$ & $5,53 \mathrm{E}+07$ & $5,39 \mathrm{E}+07$ & $3,79 \mathrm{E}+07$ & $4,68 \mathrm{E}+07$ & $5,72 \mathrm{E}+07$ & 50033,28 \\
\hline $5,91 \mathrm{E}+07$ & $5,76 \mathrm{E}+07$ & $5,65 \mathrm{E}+07$ & $4,15 \mathrm{E}+07$ & $4,95 \mathrm{E}+07$ & $5,94 \mathrm{E}+07$ & 53026,13 \\
\hline $6,22 \mathrm{E}+07$ & $5,96 \mathrm{E}+07$ & $5,86 \mathrm{E}+07$ & $4,47 \mathrm{E}+07$ & $5,16 \mathrm{E}+07$ & $6,11 \mathrm{E}+07$ & 55665,27 \\
\hline $6,44 \mathrm{E}+07$ & $6,08 \mathrm{E}+07$ & $6,14 \mathrm{E}+07$ & $4,73 \mathrm{E}+07$ & $5,30 \mathrm{E}+07$ & $6,21 \mathrm{E}+07$ & 57531,65 \\
\hline $6,59 \mathrm{E}+07$ & $6,17 \mathrm{E}+07$ & $6,21 \mathrm{E}+07$ & $4,99 \mathrm{E}+07$ & $5,40 \mathrm{E}+07$ & $6,27 \mathrm{E}+07$ & 58686,95 \\
\hline $6,68 \mathrm{E}+07$ & $6,22 \mathrm{E}+07$ & $6,24 \mathrm{E}+07$ & $5,23 \mathrm{E}+07$ & $5,46 \mathrm{E}+07$ & $6,30 \mathrm{E}+07$ & 59282,52 \\
\hline $6,72 \mathrm{E}+07$ & $6,26 \mathrm{E}+07$ & $6,25 \mathrm{E}+07$ & $5,48 \mathrm{E}+07$ & $5,49 \mathrm{E}+07$ & $6,32 \mathrm{E}+07$ & 60084,65 \\
\hline $6,74 \mathrm{E}+07$ & $6,26 \mathrm{E}+07$ & $6,25 \mathrm{E}+07$ & $5,59 \mathrm{E}+07$ & $5,51 \mathrm{E}+07$ & $6,33 \mathrm{E}+07$ & 60911,22 \\
\hline $6,73 \mathrm{E}+07$ & $6,27 \mathrm{E}+07$ & $6,25 \mathrm{E}+07$ & $5,73 \mathrm{E}+07$ & $5,54 \mathrm{E}+07$ & $6,34 \mathrm{E}+07$ & 61398,33 \\
\hline $6,71 \mathrm{E}+07$ & $6,28 \mathrm{E}+07$ & $6,25 \mathrm{E}+07$ & $5,86 \mathrm{E}+07$ & $5,58 \mathrm{E}+07$ & $6,35 \mathrm{E}+07$ & 61878,05 \\
\hline $6,70 \mathrm{E}+07$ & $6,29 \mathrm{E}+07$ & $6,26 \mathrm{E}+07$ & $5,97 \mathrm{E}+07$ & $5,62 \mathrm{E}+07$ & $6,36 \mathrm{E}+07$ & 62288,30 \\
\hline $6,70 \mathrm{E}+07$ & $6,29 \mathrm{E}+07$ & $6,27 \mathrm{E}+07$ & $6,05 \mathrm{E}+07$ & $5,66 \mathrm{E}+07$ & $6,38 \mathrm{E}+07$ & 62583,05 \\
\hline $6,70 \mathrm{E}+07$ & $6,30 \mathrm{E}+07$ & & $6,12 \mathrm{E}+07$ & $5,72 \mathrm{E}+07$ & $6,41 \mathrm{E}+07$ & 62791,91 \\
\hline & & & & $5,78 \mathrm{E}+07$ & & 62485,97 \\
\hline & & & & $5,84 \mathrm{E}+07$ & & 63054,03 \\
\hline & & & & & & 57916,38 \\
\hline
\end{tabular}




\section{ANEXO H \\ EXAMES RADIOGRÁFICOS DOS CORPOS DE PROVA DOS QUATRO GRUPOS EXPERIMENTAIS}




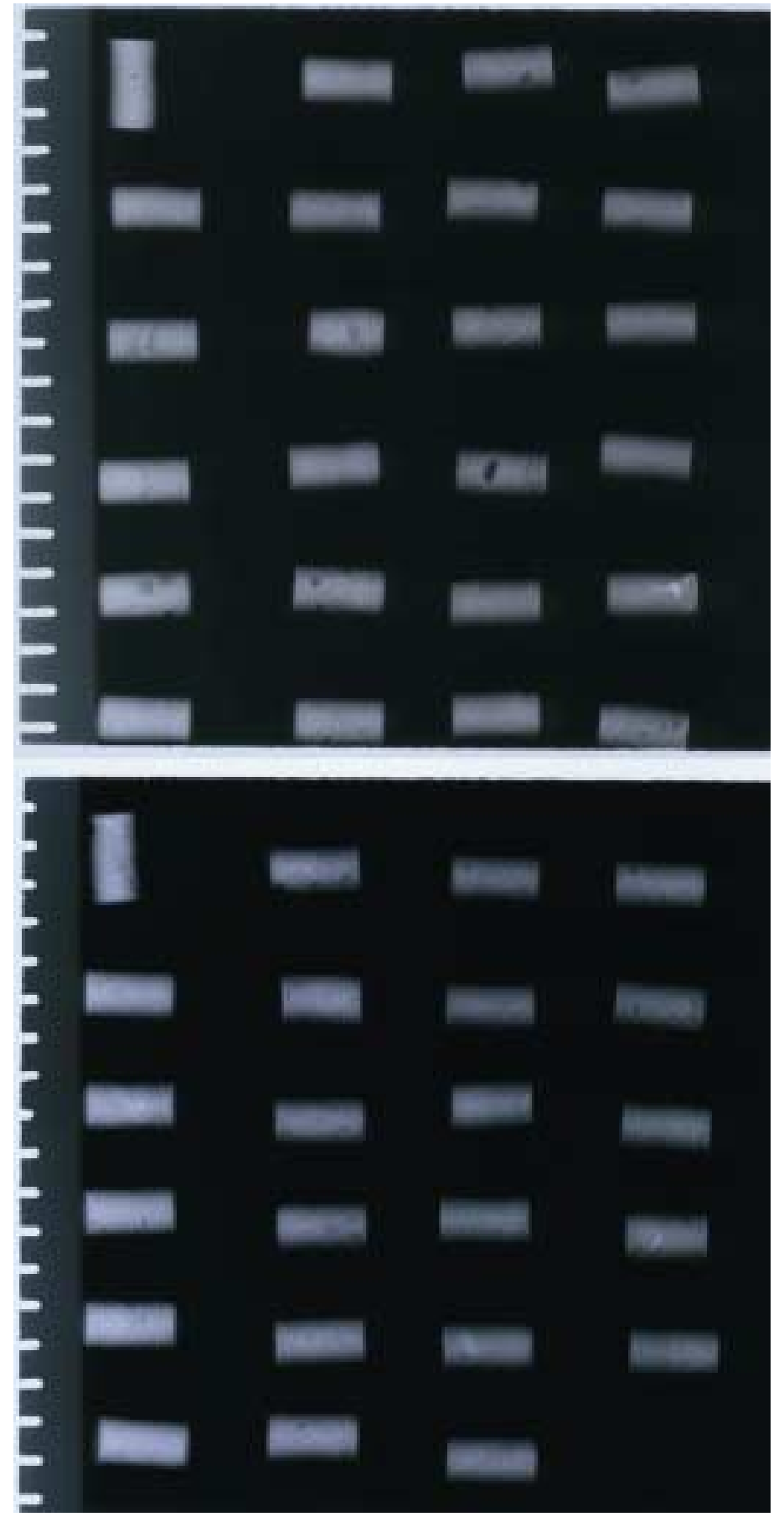

GRUPO 1M 


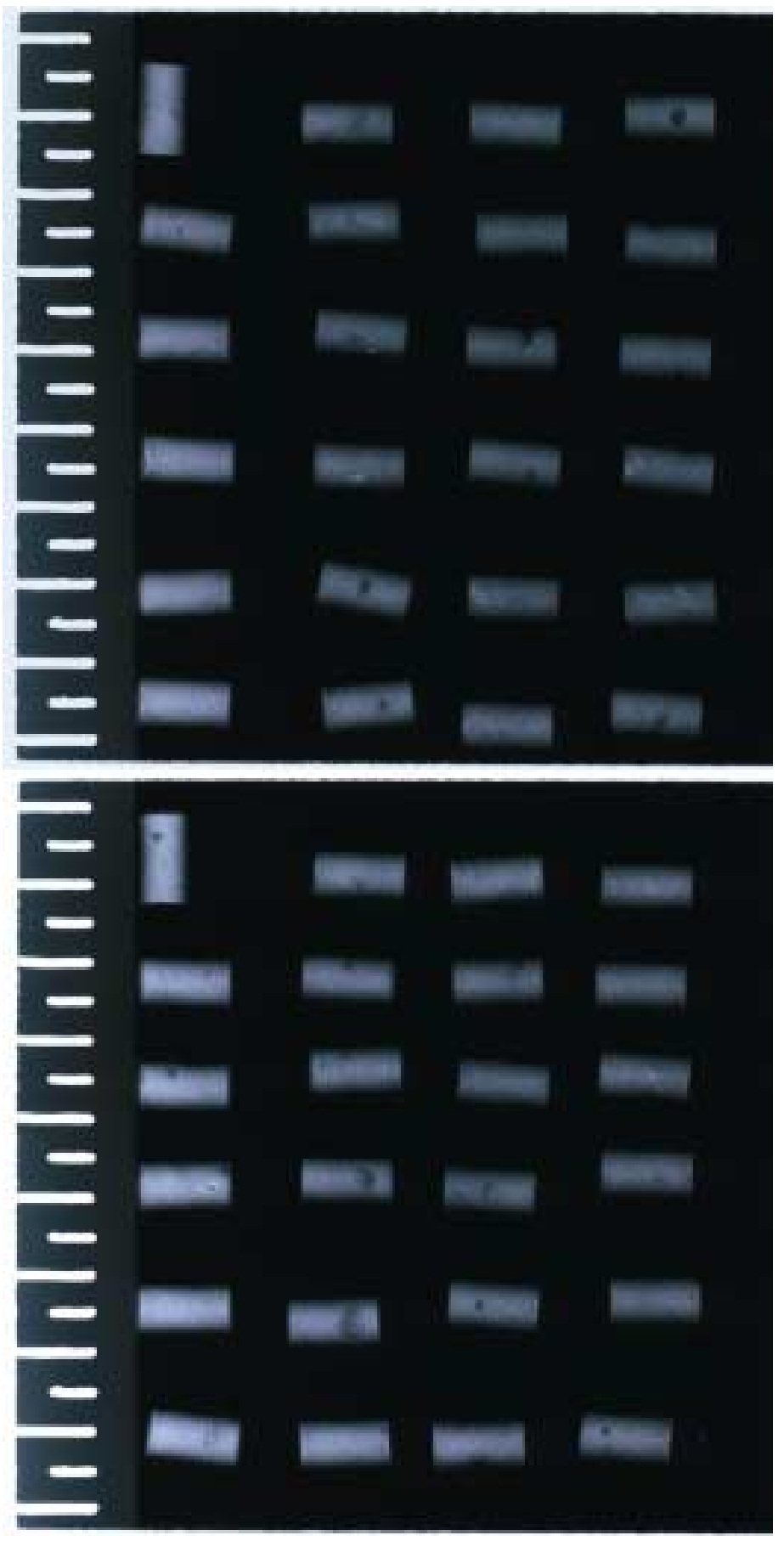

GRUPO 1V 


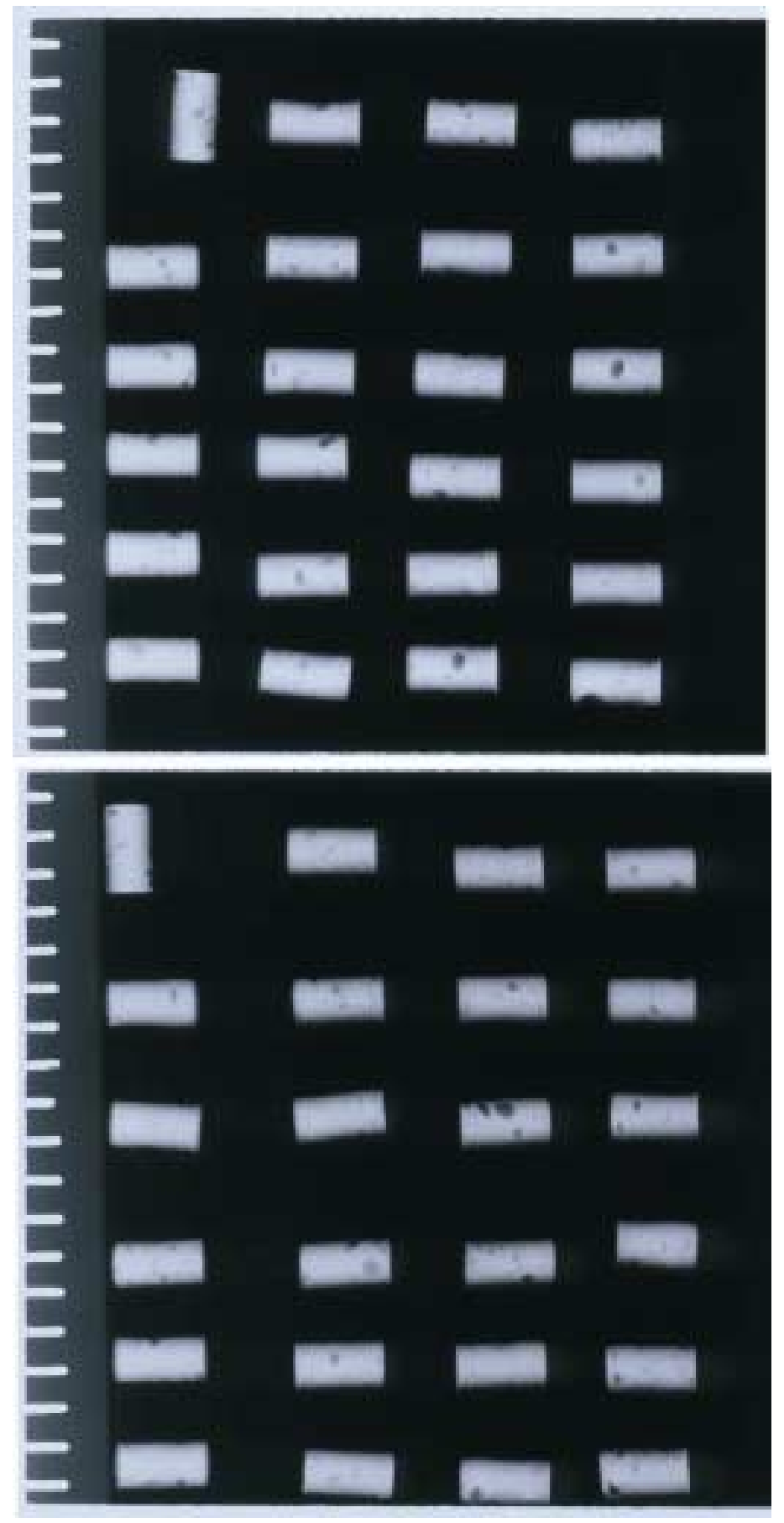

GRUPO 2M 


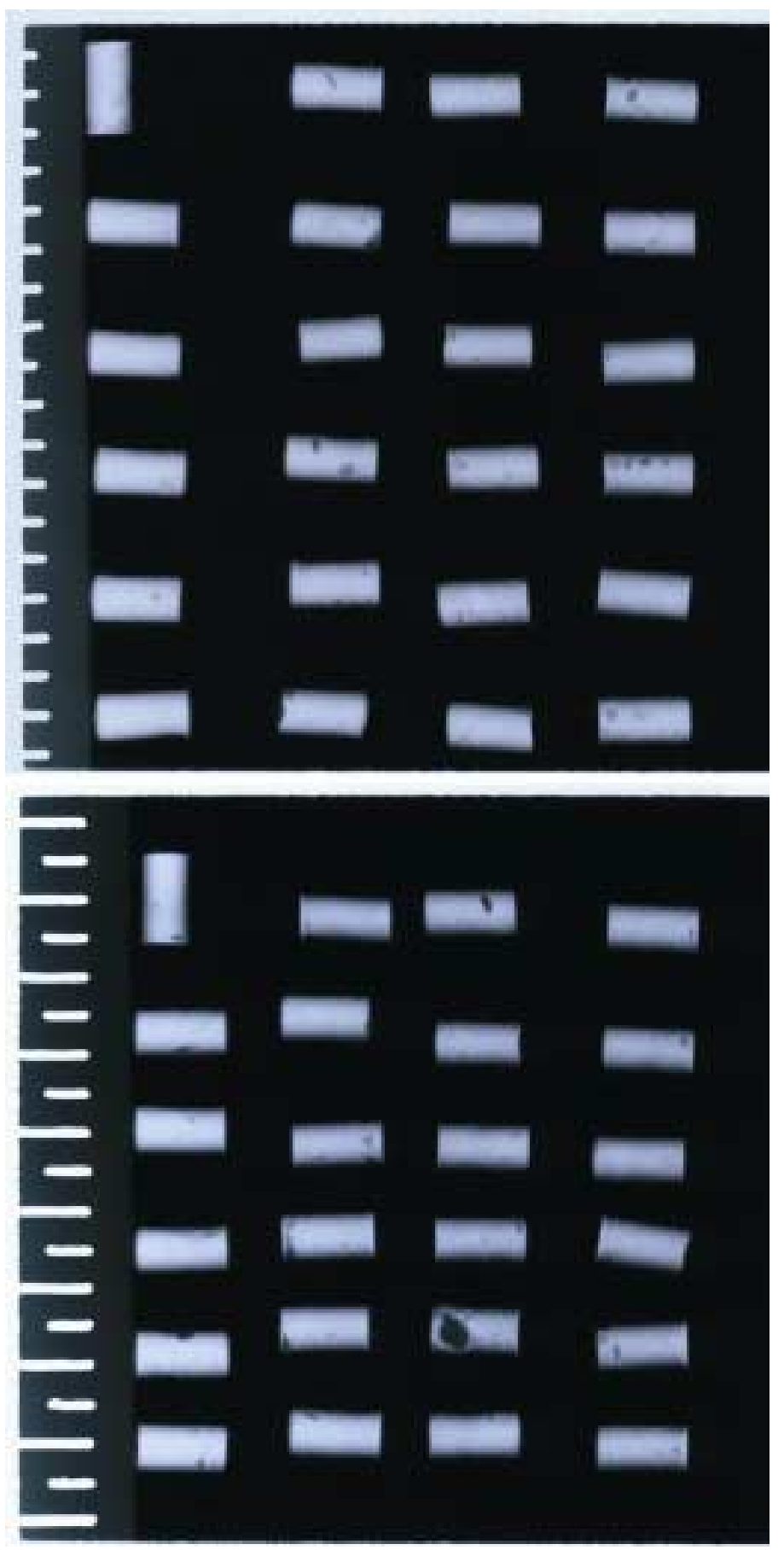

GRUPO 2V 
ANEXO I

TESTES ESTATÍSTICOS DOS DADOS DE

MÓDULO DE ELASTICIDADE 
Kruskal-Wallis One Way Analysis of Variance on Ranks

Data source: Data 1 in Notebook terça-feira, novembro $20,2001,14: 43: 54$

Normality Test: $\quad$ Failed $\quad(\mathrm{P}=<0,001)$

\begin{tabular}{llclllr} 
Group & N & Missing & \multicolumn{2}{c}{ Median } & $\mathbf{2 5 \%}$ & $\mathbf{7 5 \%}$ \\
Col 1 & $\mathbf{4 7}$ & $\mathbf{0}$ & 1389,000 & 1197,500 & 1511,000 \\
Col 2 & 47 & 0 & 1263,000 & 1200,000 & 1316,500 \\
Col 3 & 47 & 0 & 1563,000 & 1457,250 & 1641,500 \\
Col 4 & 47 & 0 & 1402,000 & 1216,250 & 1623,000
\end{tabular}

$\mathrm{H}=47,072$ with 3 degrees of freedom. $(\mathrm{P}=<0,001)$

The differences in the median values among the treatment groups are greater than would be expected by chance; there is a statistically significant difference $(\mathrm{P}=<0,001)$

To isolate the group or groups that differ from the others use a multiple comparison procedure.

All Pairwise Multiple Comparison Procedures (Dunn's Method) :

\begin{tabular}{|c|c|c|c|}
\hline Comparison & Diff of Ranks & 0 & $P<0,05$ \\
\hline $\mathrm{Col} 3$ vs $\mathrm{Col} 2$ & 75,362 & 6,714 & Yes \\
\hline Col 3 vs Col 1 & 50,585 & 4,506 & Yes \\
\hline $\mathrm{Col} 3$ vs Col 4 & 37,543 & 3,345 & Yes \\
\hline Col 4 vs Col 2 & 37,819 & 3,369 & Yes \\
\hline Col 4 vs Col 1 & 13,043 & 1,162 & No \\
\hline Col 1 vs $\mathrm{Col} 2$ & 24,777 & 2,207 & No \\
\hline
\end{tabular}

Note: The multiple comparisons on ranks do not include an adjustment for ties. 
ANEXO J

TESTES ESTATÍSTICOS DOS DADOS DA

TENSÃO NO LIMITE DE PROPORCIONALIDADE 
Data source: Data 1 in Notebook

Normality Test: $\quad$ Passed $(P>0,200)$

Equal Variance Test: Failed $(\mathrm{P}=0,001)$

$\begin{array}{llccrr}\text { Group } & \mathbf{N} & \text { Missing } & \text { Median } & \mathbf{2 5 \%} & \mathbf{7 5 \%} \\ \text { Col 1 } & 47 & 0 & 39400,000 & 35950,000 & \mathbf{4 4 9 2 5 , 0 0 0} \\ \text { Col 2 } & 47 & 0 & 36100,000 & 33925,000 & 37400,000 \\ \text { Col 3 } & 47 & 0 & 35800,000 & 31300,000 & \mathbf{4 0 0 0 0 , 0 0 0} \\ \text { Col 4 } & 47 & 0 & 39800,000 & 35950,000 & \mathbf{4 2 9 7 5 , 0 0 0}\end{array}$

$\mathrm{H}=22,653$ with 3 degrees of freedom. $(\mathrm{P}=<0,001)$

The differences in the median values among the treatment groups are greater than would be expected by chance; there is a statistically significant difference $(P=<0,001)$

To isolate the group or groups that differ from the others use a multiple comparison procedure.

All Pairwise Multiple Comparison Procedures (Dunn's Method) :

\begin{tabular}{lrrr} 
Comparison & \multicolumn{1}{c}{ Diff of Ranks } & $\mathbf{Q}$ & $\mathbf{P}<0,05$ \\
Col 1 vs Col 3 & 41,819 & 3,726 & Yes \\
Col 1 vs Col 2 & 40,521 & 3,610 & Yes \\
Col 1 vs Col 4 & 7,574 & 0,675 & No \\
Col 4 vs Col 3 & 34,245 & 3,051 & Yes \\
Col 4 vs Col 2 & 32,947 & 2,935 & Yes \\
Col 2 vs Col 3 & 1,298 & 0,116 & No
\end{tabular}

Note: The multiple comparisons on ranks do not include an adjustment for ties. 
ANEXO L

TESTES ESTATÍSTICOS DOS DADOS DA

DEFORMAÇÃO NO LIMITE DE PROPORCIONALIDADE 
Kruskal-Wallis One Way Analysis of Variance on Ranks

Data source: Data 1 in Notebook

Normality Test: $\quad$ Failed $\quad(\mathrm{P}=<0,001)$

$\begin{array}{llclll}\text { Group } & \mathbf{N} & \text { Missing } & \text { Median } & \mathbf{2 5 \%} & \mathbf{7 5 \%} \\ \text { Col 1 } & \mathbf{4 7} & \mathbf{0} & 3,360 & 2,515 & 3,777 \\ \text { Col 2 } & \mathbf{4 7} & 0 & 2,950 & 2,538 & 3,237 \\ \text { Col 3 } & \mathbf{4 7} & \mathbf{0} & 2,530 & 2,120 & 2,567 \\ \text { Col 4 } & \mathbf{4 7} & \mathbf{0} & 2,930 & 2,695 & 3,357\end{array}$

$\mathrm{H}=31,972$ with 3 degrees of freedom. $(\mathrm{P}=<0,001)$

The differences in the median values among the treatment groups are greater than would be expected by chance; there is a statistically significant difference $(P=<0,001)$

To isolate the group or groups that differ from the others use a multiple comparison procedure.

All Pairwise Multiple Comparison Procedures (Dunn's Method) :

\begin{tabular}{|c|c|c|c|}
\hline Comparison & Diff of Ranks & C & $P<0,05$ \\
\hline Col 1 vs $\mathrm{Col} 3$ & $-59,298$ & 5,283 & Yes \\
\hline Col 1 vs Col 2 & 15,904 & 1,417 & No \\
\hline Col 1 vs Col 4 & 11,989 & 1,068 & Do Not Test \\
\hline Col 4 vs Col 3 & 47,309 & 4,215 & Yes \\
\hline $\mathrm{Col} 4$ vs $\mathrm{Col} 2$ & 3,915 & 0,349 & Do Not Test \\
\hline Col 2 vs Col 3 & 43,394 & 3,866 & Yes \\
\hline
\end{tabular}

Note: The multiple comparisons on ranks do not include an adjustment for ties. 